UNIVERSIDAD POLITÉCNICA DE MADRID

ESCUELA TÉCNICA SUPERIOR DE INGENIEROS DE MONTES, FORESTAL Y DEL MEDIO NATURAL
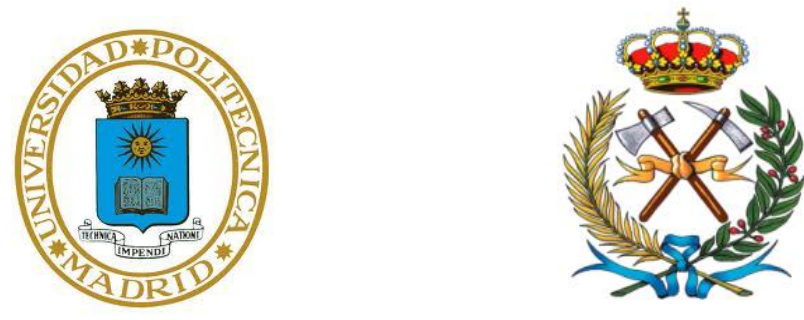

DESARROLLO DE UN MODELO DE PLANIFICACIÓN ENERGÉTICA SOSTENIBLE USANDO TÉCNICAS DE OPTIMIZACIÓN MULTICRITERIO: UNA APLICACIÓN A LA PROVINCIA DE CAJAMARCA, PERÚ

TESIS DOCTORAL

Eder Jesús Falcón Roque

Ingeniero Agrícola 
PROGRAMA DE DOCTORADO EN INVESTIGACIÓN FORESTAL AVANZADA

ESCUELA TÉCNICA SUPERIOR DE INGENIEROS DE MONTES, FORESTAL Y DEL MEDIO NATURAL

\title{
DESARROLLO DE UN MODELO DE PLANIFICACIÓN ENERGÉTICA SOSTENIBLE USANDO TÉCNICAS DE OPTIMIZACIÓN MULTICRITERIO: UNA APLICACIÓN A LA PROVINCIA DE CAJAMARCA, PERÚ
}

\author{
Eder Jesús Falcón Roque \\ Ingeniero Agrícola
}

\section{DIRECTOR}

Isabel Cristina Pascual Castaño

Doctor Ingeniero de Montes 
POLITÉCNICA

Tribunal nombrado por el Excmo. Sr. Rector de la Universidad Politécnica de Madrid, el día de de 2018.

Presidente D.

Vocal D.

Vocal D

Vocal D.

Secretario D

Realizado el acto de defensa y lectura de Tesis el día de de 2018 en Madrid.

Calificación:

EL PRESIDENTE

LOS VOCALES

EL SECRETARIO 


\section{AGRADECIMIENTOS}

A Dios, quien me dio el ser y la capacidad de amar y de recibir amor.

A los Cruzados de Santa María, mi familia espiritual, por ser hogar de fraternidad, comunión y misericordia.

A mis padres, hermanos y sobrinos, por su incondicionalidad, por ser fuente de motivación y por recordarme siempre, a pesar de la distancia.

A Cristina y a Paco, sin su apoyo profesional y académico, no habría sido posible terminar este trabajo.

A los que colaboraron conmigo en la publicación del artículo científico producto de esta tesis doctoral: Cristina, Paco, Luis Carlos y Javier, ha sido un lujo trabajar con vosotros.

A Julio y a Pepo, de la Fundación ACCIONA Microenergía, quienes han sido maestros en la acción social en beneficio de las comunidades rurales menos favorecidas de Latinoamérica a través de energías renovables, gracias por vuestra profesionalidad y entusiasmo.

A Jessica y a todo el equipo de ACCIONA Microenergía Perú, por esa entrega diaria, que implica, a veces, horas de caminata a 3.000 metros de altura, viajes en camioneta por carreteras poco accesibles, exponerse a algún canino poco amigable... todo por llevar luz eléctrica a las comunidades rurales aisladas de Perú a través de energías limpias. 


\section{RESUMEN}

Tomar una decisión en planificación energética implica un proceso de equilibrar diversos aspectos, tales como los ambientales, sociales, técnicos y económicos. Este equilibrio es muy importante para alcanzar lo que hoy se conoce como desarrollo sostenible.

La presente tesis doctoral tiene por objetivo desarrollar un modelo de planificación energética con energías renovables basado en técnicas de optimización multicriterio que, además de considerar aspectos económicos y medio ambientales, tenga en cuenta el suministro sostenible de energía en las comunidades rurales aisladas, problema que en Latinoamérica es de especial relevancia.

El primer paso para el desarrollo del modelo fue proponer un estudio exhaustivo de la oferta y demanda energéticas por sectores de producción, asimismo, del potencial de las fuentes energéticas renovables. En esta etapa se tuvo en cuenta la proyección de la demanda para el año horizonte de la planificación (año 2025). Posteriormente fueron definidas las restricciones y las alternativas energéticas a considerar en el estudio. Para ello, han sido consideradas como fundamentales las reuniones entre los decisores y los técnicos, lo cual orienta la planificación hacia un adecuado aprovechamiento de los recursos y permite una priorización de las tecnologías con mayor potencial de uso.

El modelo propuesto, denominado SEPLAN (Sustainable Energy Planning), ha establecido seis funciones objetivo: sustitución de energía fósil por energías renovables, minimización de costes, acceso universal a la energía en comunidades rurales aisladas y las emisiones evitadas de $\mathrm{CO}_{2}, \mathrm{NO}_{x}$ y $\mathrm{SO}_{2}$, respectivamente. Definidas las funciones objetivo, se ha realizado la optimización independiente de cada función. Finalmente, se obtiene el conjunto de soluciones eficientes a través de la aplicación de la técnica multicriterio Programación Compromiso, eligiendo a la distancia Chebyshev $\left(\mathrm{L}_{\infty}\right)$ como solución óptima. De este modo, el modelo SEPLAN incorpora en su formulación objetivos que podrían estar en conflicto, por ejemplo, objetivos económicos, ambientales, sociales, o especialmente importantes para un país de Latinoamérica, como podría ser el acceso universal a la energía, convirtiéndose en una herramienta útil para los centros decisores que busquen asegurar el bienestar de las generaciones futuras. 
El modelo SEPLAN se ha aplicado en la provincia de Cajamarca (Perú), que tiene la tasa de electrificación más baja del país debido a la alta dispersión de las viviendas en las comunidades rurales. Se analizaron diversos escenarios, los cuales fueron establecidos según el peso asignado a cada función objetivo, obteniéndose la priorización de las alternativas energéticas consideradas. Al potenciar la función objetivo relacionada con el acceso universal a la energía en comunidades rurales aisladas, la energía solar fotovoltaica fue la prioridad en la generación de energía eléctrica. Finalmente, fue realizado un análisis de sensibilidad considerando una variación en el precio del petróleo y en el Producto Interno Bruto (PIB), obteniéndose el comportamiento esperado de las variables energéticas estudiadas. 


\section{ABSTRACT}

Making a decision in energy planning involves a process of balancing various aspects, such as environmental, social, technical and economic ones. This balance is very important for achieving sustainable development.

The aim of this doctoral thesis is to perform an energy planning model with renewable energies and based on multi-criteria optimization techniques. This model considers, in additional to economics and environmental aspects, the sustainable supply of energy in isolated rural communities which is a relevant issue in Latin America.

The first step was to perform a comprehensive review of supply and demand energy in the different production sectors, likewise, the renewable energies potential. This step takes into account the forecast demand for the planning horizon (year 2025). Then, restrictions and energy alternatives to consider in the study was defined. For this purpose, the meetings of the decision makers and experts were essential, since they led the study towards an appropriate use of resources and allowed prioritization of the technologies with most use potential.

The model proposed, named SEPLAN (Sustainable Energy Planning), has established six objective functions: substitution of fossil energy by renewable energies, cost minimization, universal access to energy in isolated rural communities and $\mathrm{CO}_{2}$, $\mathrm{NO} x$ and $\mathrm{SO}_{2}$ avoidable emissions, respectively. Once the objective functions were defined, the independent optimization of each function was performed. Finally, the set of efficient solutions were obtained applying Compromise Programing multi-criteria technique and Chebyshev distance $\left(\mathrm{L}_{\infty}\right)$ was chosen as optimal solution. Thereby, SEPLAN model has allowed incorporate objective that could be in conflict, i.e. economics, environmental, social or the universal energy access ones. Thus, it could become in an useful tool for decision makers that seek ensure the well-being of future generations.

SEPLAN model was applied in Cajamarca province (Peru). Cajamarca region has the lowest electrification rate of the country due to the high dispersion of households in their rural communities. Several scenarios were analyzed. These scenarios were established according to the weight given to each objective function, thereby, the prioritization of the energy alternatives was obtained. By prioritizing the objective 
function related to universal energy access, photovoltaic solar energy was the most important for electric power generation. Finally, a sensitivity analysis was carried out that considered a variation in the price of oil and in the Gross Domestic Product (GDP). These results showed that the energy variables studied had the expected behavior. 


\section{ÍNDICE DE LA MEMORIA}

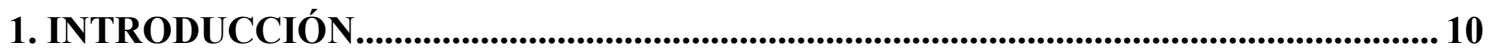

1.1 Energía, desarrollo sostenible y acceso universal a la energía. ................................ 10

1.2 La situación energética de Perú .......................................................................... 15

1.3 Planificación energética en entornos rurales aislados. Técnicas multicriterio.

Región de Cajamarca............................................................................................................... 19

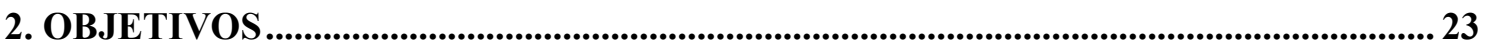

2.1 Objetivo General ............................................................................................................... 23

2.2 Objetivos Específicos............................................................................................................ 23

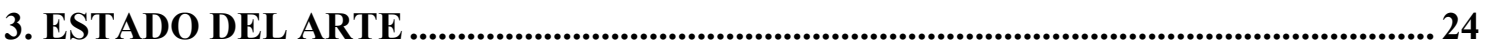

3.1 Métodos multicriterio usados en planificación energética....................................... 25

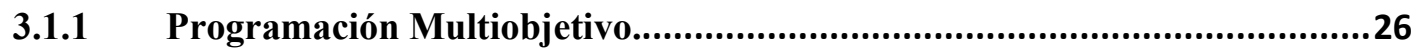

3.1.1.1 La matriz de pagos en la programación multiobjetivo .................................... 26

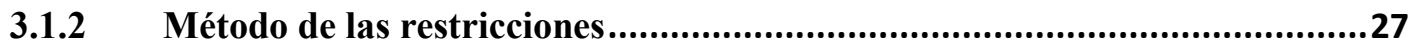

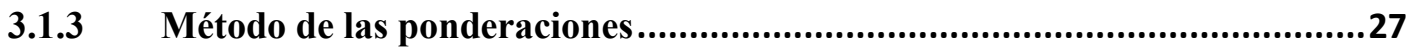

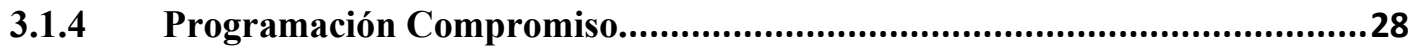

3.1.4.1 Determinación de las soluciones compromiso........................................... 28

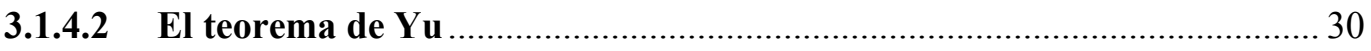

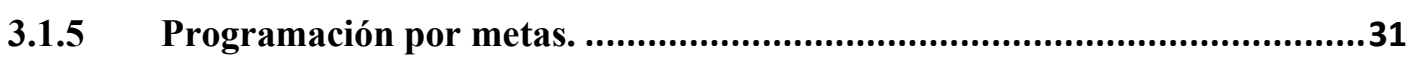

3.1.5.1 Estructura general de un modelo de programación por metas................. 32

3.1.6 Proceso de Jerarquías Analíticas ..............................................................33

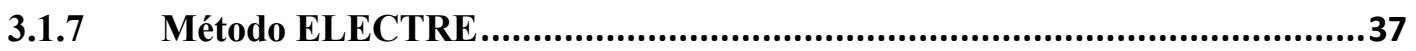

3.1.8 Técnicas de análisis de información (Técnicas blandas)..............................38

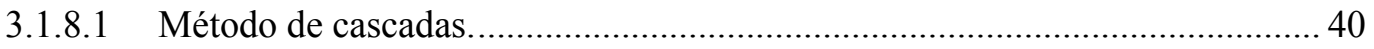

3.1.8.2 Método del producto de componentes.......................................................... 42

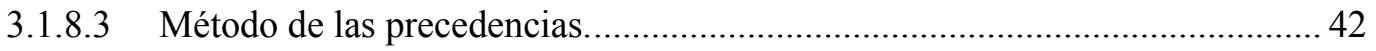

3.1.8.4 Método de la distancia al punto ideal.............................................................. 43

3.1.9 Programación no lineal..............................................................................44

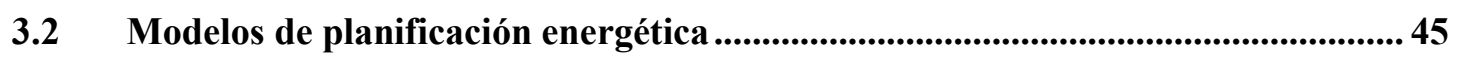

3.2.1 Modelos de demanda energética ...............................................................48

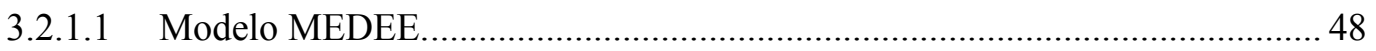

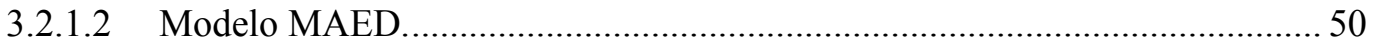

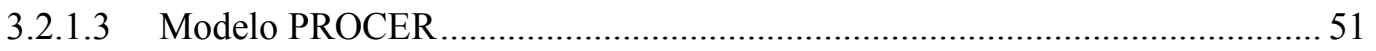

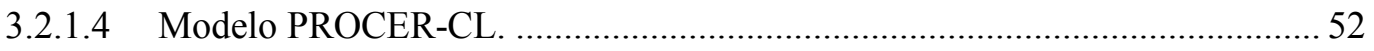

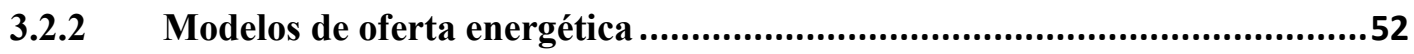

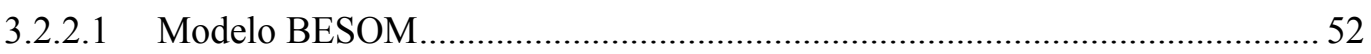




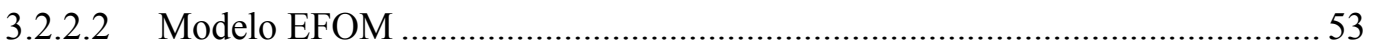

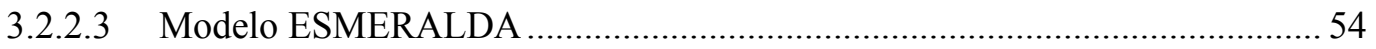

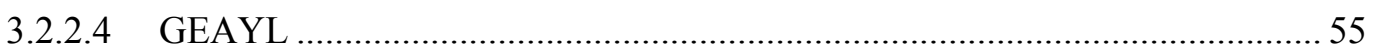

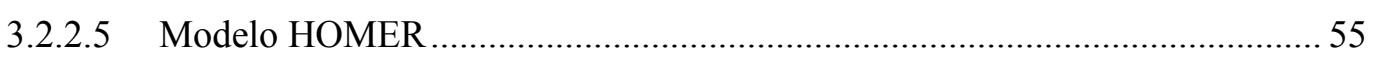

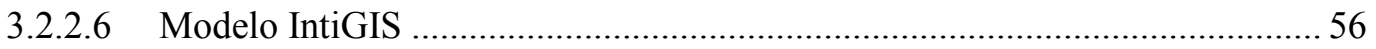

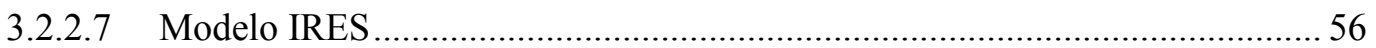

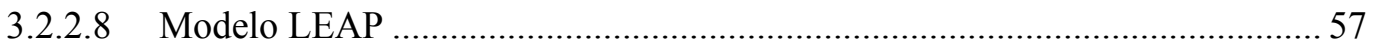

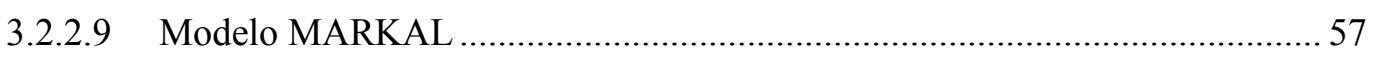

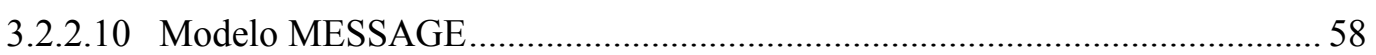

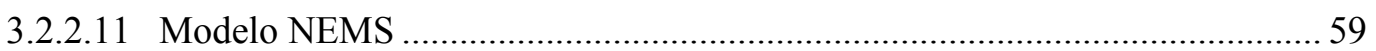

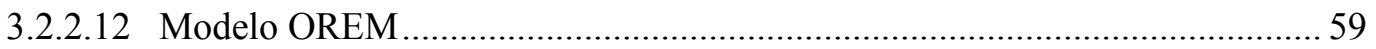

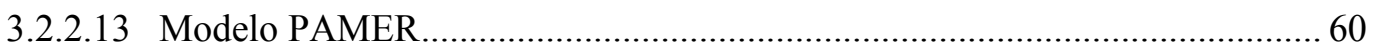

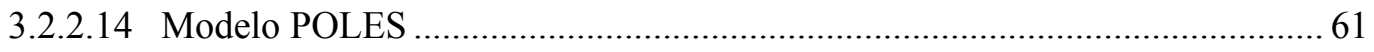

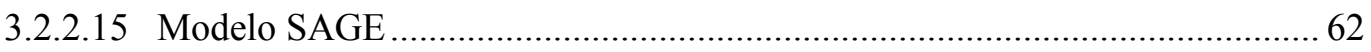

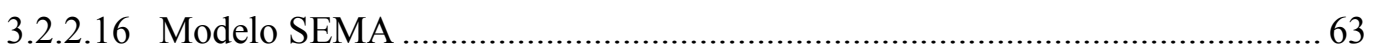

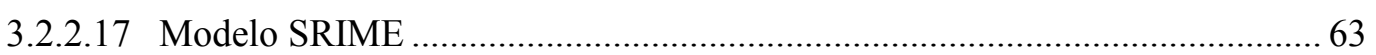

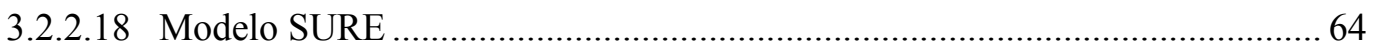

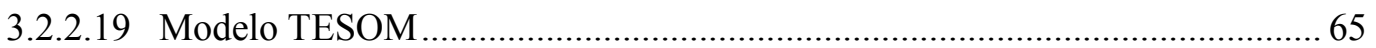

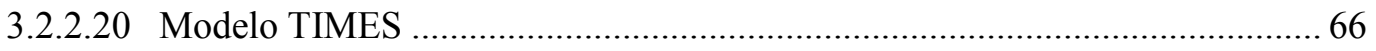

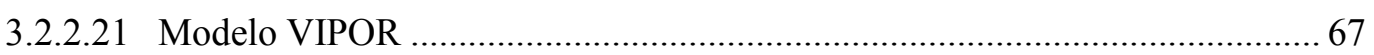

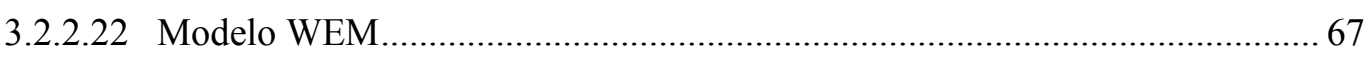

4. PROPUESTA DE UN MODELO DE PLANIFICACIÓN ENERGÉTICA ..................... 72

4.1 Modelo propuesto: Modelo de planificación energética SEPLAN......................... 72

4.2 Diagnostico situacional de la zona de estudio. Estudio de Oferta y Demanda..... 74

4.2.1 Estimación de la Demanda .......................................................................74

4.2.2 Estimación de la Oferta ..........................................................................74

4.3 Determinación de las alternativas energéticas............................................................... 74

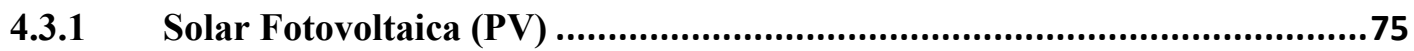

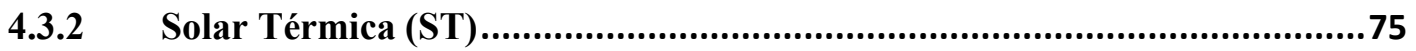

4.3.3 Energía Eólica Baja Potencia (EOL) …..................................................75

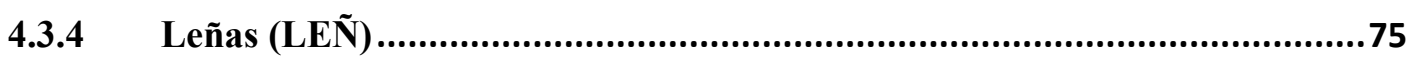

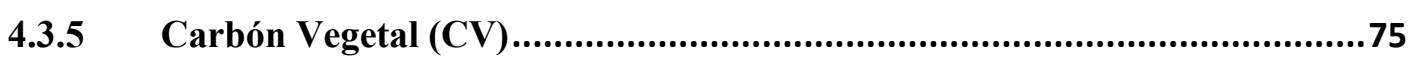

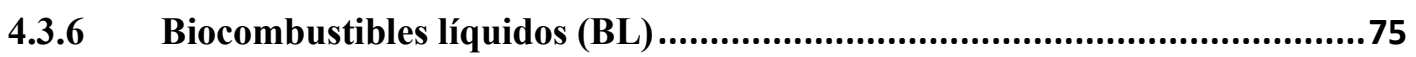

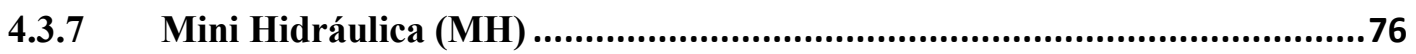

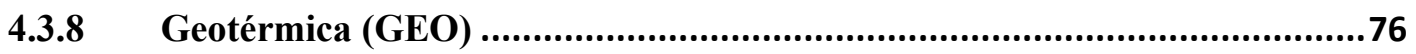

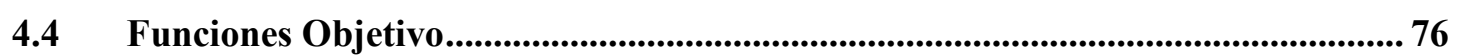


4.4.1 $\quad F_{1}$ : Maximización de la sustitución de las energías fósiles por energías

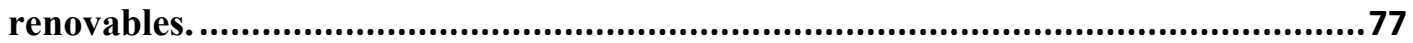

4.4.2 $\quad \mathrm{F}_{2}, \mathrm{~F}_{3}$ y $\mathrm{F}_{4}$ : Maximización de las emisiones evitadas de $\mathrm{CO}_{2}, \mathrm{NO}_{\mathrm{x}} \mathrm{y} \mathrm{SO}_{2}$,

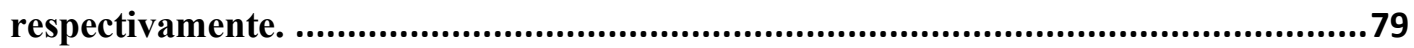

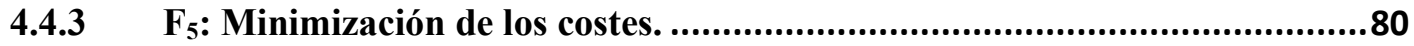

4.4.4 $\quad F_{6}$ : Maximización del uso de alternativas energéticas renovables para el suministro sostenible de energía en comunidades rurales aisladas. ..........................81

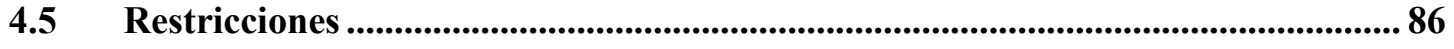

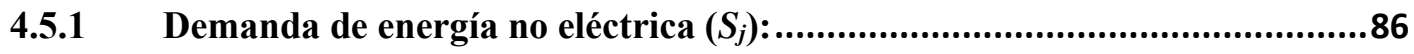

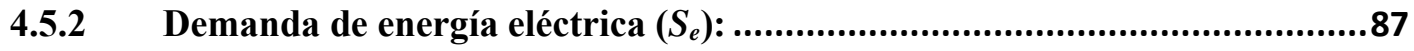

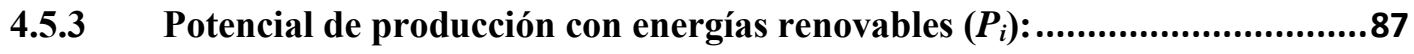

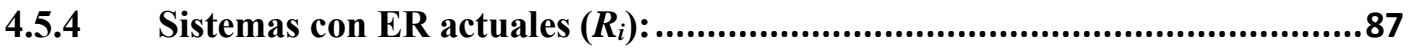

4.6 Programación Compromiso ............................................................................................... 88

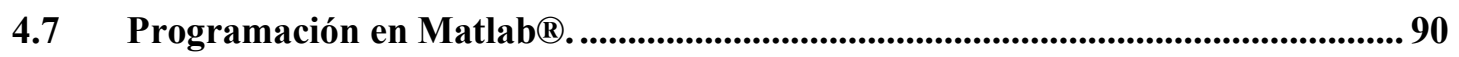

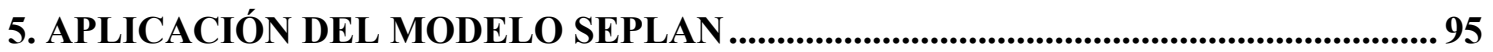

5.1 Descripción de la zona de estudio. Provincia de Cajamarca.................................... 95

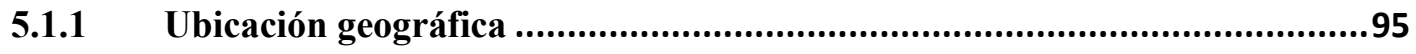

5.1.2 Características principales .......................................................................97

5.2 Estudio de la demanda y oferta energéticas.................................................................. 97

5.2.1 Estimación de la demanda energética...........................................................97

5.2.2 Estimación de la oferta energética ............................................................101

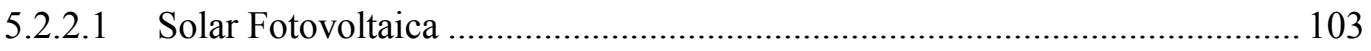

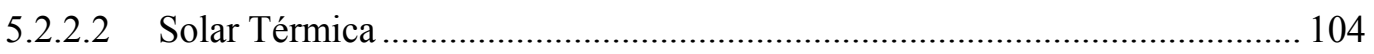

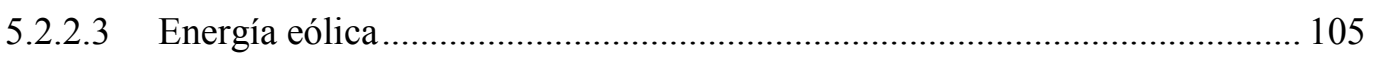

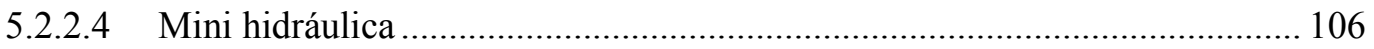

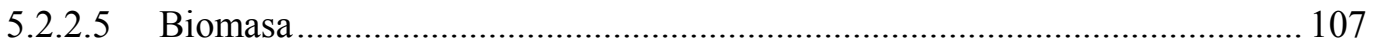

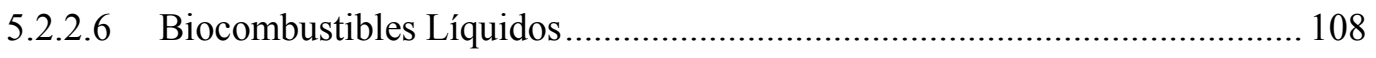

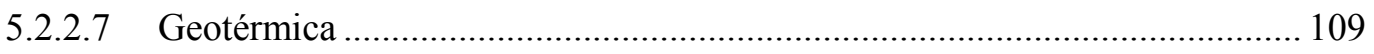

5.3 Establecimiento de las restricciones .............................................................. 109

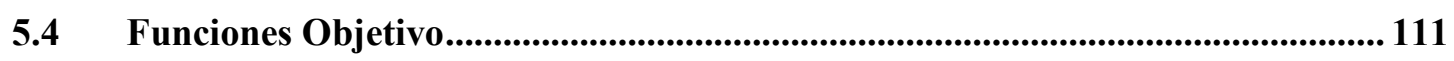

5.4.1 $\quad F_{1}$ : Maximización de la sustitución de las energías fósiles por energías

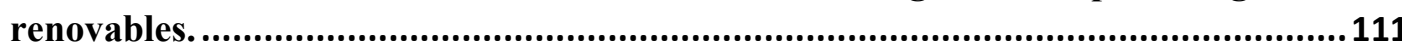

5.4.2 $\quad \mathrm{F}_{2}, \mathrm{~F}_{3}$ y $\mathrm{F}_{4}$ : Maximización de las emisiones evitadas de $\mathrm{CO}_{2}, \mathrm{NO}_{\mathrm{x}}$ y $\mathrm{SO}_{2}$,

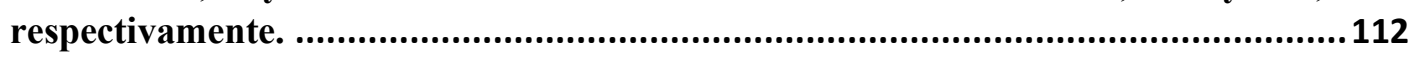

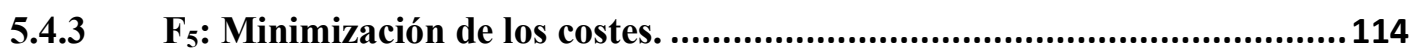

5.4.4 Foximización del uso de alternativas energéticas renovables para el suministro sostenible de energía en CRA. .............................................................115

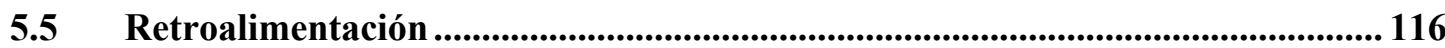


6.1 Análisis de la variación de las alternativas energéticas para las distancias calculadas.

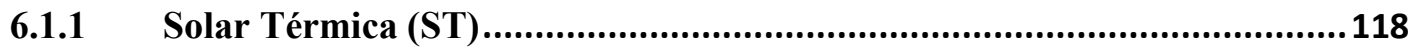

6.1.2 Solar Fotovoltaica (PV) ........................................................................119

6.1.3 Energía Eólica (EOL) ….................................................................120

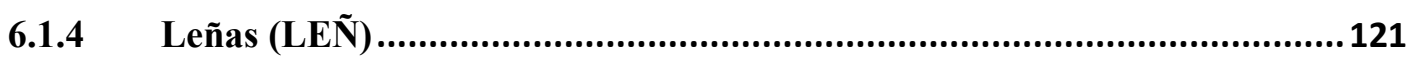

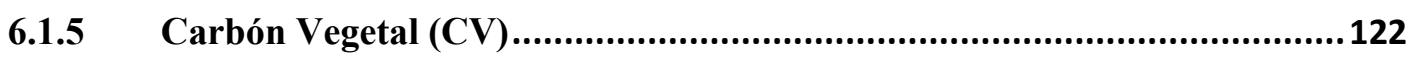

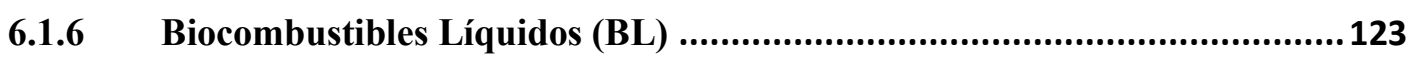

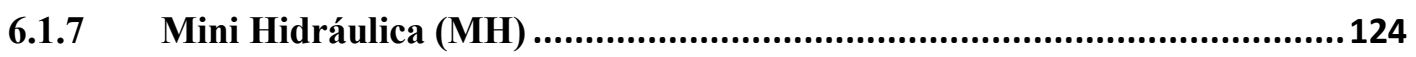

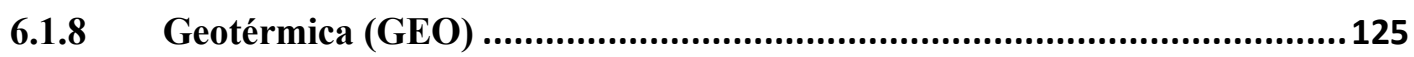

6.2 Análisis de la variación de las funciones objetivo para las distancias calculadas. 126

6.2.1 $\quad F_{1}$ : Maximización de la sustitución de las energías fósiles por energías renovables. 126

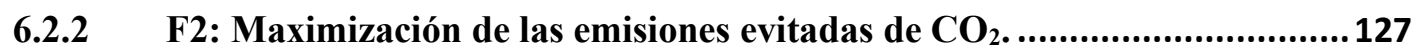

6.2.3 $\quad F_{3}$ : Maximización de las emisiones evitadas de $\mathrm{NO}_{\mathrm{x}}$...............................128

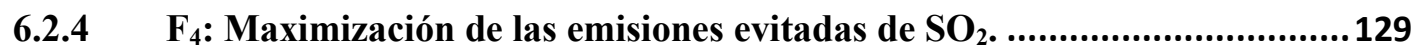

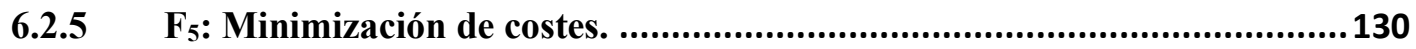

6.2.6 $\quad F_{6}$ : Maximización del uso de alternativas energéticas renovables para el suministro sostenible de energía en CRA.

6.3 Análisis de la variación de las funciones objetivo para la distancia Chebyshev $(\mathbf{L} \infty) .132$

6.4 Análisis de sensibilidad de los resultados..................................................................... 140

6.5 Comparativa del modelo SEPLAN, con los modelos PAMER y SEMA. ............. 144

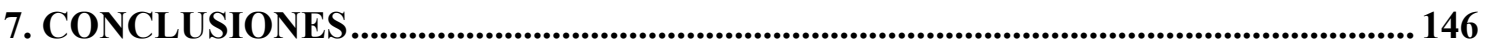

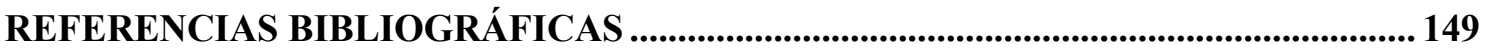

ANEXO I:_DATOS OBTENIDOS DEL PROGRAMA MATLAB ${ }^{\circledR}$ PARA CADA

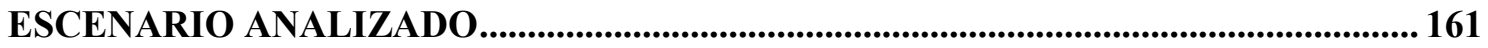

ANEXO II:_DATOS OBTENIDOS POR VARIABLE Y FUNCIÓN OBJETIVO............ 167

ANEXO III:_DATOS OBTENIDOS DEL ANÁLISIS DE SENSIBILIDAD...................... 175

ANEXO IV:_PAPER PUBLICADO EN LA REVISTA "JOURNAL OF RENEWABLE

AND SUSTAINABLE ENERGY".......................................................................................... 179 


\section{LISTA DE FIGURAS}

Figura 1-1. Consumo de energía en los Estados Unidos (1776-2014) en $10^{15}$ BTU (U.S. Energy Information Administration, 2015)

Figura 1-2. Perspectivas de crecimiento de la demanda mundial de energía primaria (International Energy Agency, 2016).

Figura 1-3. Desarrollo sostenible.

Figura 1-4. Acceso universal a la electricidad al 2014 (\% de población) (World Bank, 2014) 14

Figura 1-5. Objetivos de Desarrollo Sostenible (Naciones Unidas, 2015)..... 16

Figura 1-6. Consumo de Energía Primaria en Perú en el año 2014 (OSINERGMIN, 2015b)

Figura 1-7. Fuentes energéticas para la generación de energía eléctrica en Perú. Día de máxima demanda. 14 de Noviembre, 2016 (Delta Volt, 2016). 18

Figura 3-1. Modelo de Jerarquías Analíticas (Parodi, 2013). 34

Figura 4-1. Diagrama del modelo SEPLAN. 73

Figura 4-2. Coeficientes de electrificación nacional en Perú al 2011. Fuente: MINEM (2012). 82

Figura 4-3. Árbol de Medios y Fines en proyectos de electrificación rural (ACCIONA Microenergía Perú, 2011). 83

Figura 5-1. Ubicación geográfica de la provincia de Cajamarca. 96

Figura 5-2. Mapa político de la provincia de Cajamarca (Pontificia Universidad Católica del Perú, 2007). 96

Figura 5-3. Proyección del consumo eléctrico del 2015 al 2025 en la provincia de Cajamarca, para un incremento del PBI del 6.5\%.

Figura 5-4. Disponibilidad de energía solar diaria en el departamento de Cajamarca para los meses de febrero, mayo, agosto y noviembre en $\mathrm{kWh} / \mathrm{m}^{2}$ (Servicio Nacional de Meteorología e Hidrología, 2003).

Figura 5-5. Velocidad del viento anual a $80 \mathrm{~m}$ en el departamento de Cajamarca (Ministerio de Energía y Minas, 2008). 106

Figura 6-1. Variación de ST según los escenarios estudiados y para las distancias $L_{1}$, $\mathrm{L}_{2}, \mathrm{~L}_{3}$ y $\mathrm{L}_{\infty}$. 119 
Figura 6-2. Variación de PV según los escenarios estudiados y para las distancias $\mathrm{L}_{1}$, $\mathrm{L}_{2}, \mathrm{~L}_{3}$ y $\mathrm{L}_{\infty}$. 120

Figura 6-3. Variación de EOL según los escenarios estudiados y para las distancias $\mathrm{L}_{1}$, $\mathrm{L}_{2}, \mathrm{~L}_{3}$ y $\mathrm{L}_{\infty}$

Figura 6-4. Variación de LEÑ según los escenarios estudiados y para las distancias $\mathrm{L}_{1}$, $\mathrm{L}_{2}, \mathrm{~L}_{3} \mathrm{y} \mathrm{L}_{\infty}$

Figura 6-5. Variación de CV según los escenarios estudiados y para las distancias $L_{1}$, $\mathrm{L}_{2}, \mathrm{~L}_{3}$ y $\mathrm{L}_{\infty}$. 123

Figura 6-6. Variación de BL según los escenarios estudiados y para las distancias $\mathrm{L}_{1}$, $\mathrm{L}_{2}, \mathrm{~L}_{3}$ y $\mathrm{L}_{\infty}$.

Figura 6-7. Variación de MH según los escenarios estudiados y para las distancias $\mathrm{L}_{1}$, $\mathrm{L}_{2}, \mathrm{~L}_{3}$ y $\mathrm{L}_{\infty}$ 125

Figura 6-8. Variación de GEO según los escenarios estudiados y para las distancias $\mathrm{L}_{1}$, $\mathrm{L}_{2}, \mathrm{~L}_{3}$ y $\mathrm{L}_{\infty}$. 126

Figura 6-9. Variación de $\mathrm{F}_{1}$ según los escenarios estudiados y para las distancias $\mathrm{L}_{1}, \mathrm{~L}_{2}$, $\mathrm{L}_{3}$ y $\mathrm{L}_{\infty}$

Figura 6-10. Variación de $\mathrm{F}_{2}$ según los escenarios estudiados y para las distancias $\mathrm{L}_{1}$, $\mathrm{L}_{2}, \mathrm{~L}_{3}$ y $\mathrm{L}_{\infty}$

Figura 6-11. Variación de $\mathrm{F}_{3}$ según los escenarios estudiados y para las distancias $\mathrm{L}_{1}$, $\mathrm{L}_{2}, \mathrm{~L}_{3}$ y $\mathrm{L}_{\infty}$.

Figura 6-12. Variación de $\mathrm{F}_{4}$ según los escenarios estudiados y para las distancias $\mathrm{L}_{1}$,

$\mathrm{L}_{2}, \mathrm{~L}_{3}$ y $\mathrm{L}_{\infty}$. 130

Figura 6-13. Variación de $\mathrm{F}_{5}$ según los escenarios estudiados y para las distancias $\mathrm{L}_{1}$, $\mathrm{L}_{2}, \mathrm{~L}_{3}$ y $\mathrm{L}_{\infty}$.

Figura 6-14. Variación de $\mathrm{F}_{6}$ según los escenarios estudiados y para las distancias $\mathrm{L}_{1}$,

$\mathrm{L}_{2}, \mathrm{~L}_{3}$ y $\mathrm{L}_{\infty}$

Figura 6-15. Resultado de la optimización no ponderada para cada alternativa energética. Distancia Chebyshev $\left(\mathrm{L}_{\infty}\right)$.

Figura 6-16. Resultados de la priorización de $F_{1}$ para cada alternativa energética.

Distancia Chebyshev $\left(\mathrm{L}_{\infty}\right)$.

Figura 6-17. Resultados de la priorización de $F_{2}$ para cada alternativa energética.

Distancia Chebyshev $\left(\mathrm{L}_{\infty}\right)$.

Figura 6-18. Resultados de la priorización de $\mathrm{F}_{3}$ para cada alternativa energética.

Distancia Chebyshev $\left(\mathrm{L}_{\infty}\right)$. 
Figura 6-19. Resultados de la priorización de $\mathrm{F}_{4}$ para cada alternativa energética.

Distancia Chebyshev $\left(\mathrm{L}_{\infty}\right)$

Figura 6-20. Resultados de la priorización de $\mathrm{F}_{5}$ para cada alternativa energética.

Distancia Chebyshev $\left(\mathrm{L}_{\infty}\right)$.

Figura 6-21. Resultados de la priorización de $\mathrm{F}_{6}$ para cada alternativa energética.

Distancia Chebyshev $\left(\mathrm{L}_{\infty}\right)$.

Figura 6-22. Resultados de la priorización de $F_{2}, F_{3}$ y $F_{4}$ para cada alternativa energética. Distancia Chebyshev $\left(\mathrm{L}_{\infty}\right)$.

Figura 6-23. Resultados de la priorización de $\mathrm{F}_{1}$ y $\mathrm{F}_{6}$ para cada alternativa energética.

Distancia Chebyshev $\left(\mathrm{L}_{\infty}\right)$.

Figura 6-24. Resultados del análisis de sensibilidad para LEÑ con incremento del precio de petróleo en 50\% para los distintos escenarios. (Distancia $\mathrm{L}_{\infty}$ ).

Figura 6-25. Resultados del análisis de sensibilidad para CV con incremento del precio de petróleo en $50 \%$ para los distintos escenarios. (Distancia $\mathrm{L}_{\infty}$ ).

Figura 6-26. Resultados del análisis de sensibilidad para BL con incremento del precio de petróleo en $50 \%$ para los distintos escenarios. (Distancia $\mathrm{L}_{\infty}$ ).

Figura 6-27. Resultados del análisis de sensibilidad con incremento del precio de petróleo en $50 \%$ para las alternativas energéticas consideradas sin priorización de funciones

Figura 6-28. Resultados del análisis de sensibilidad con disminución del PBI a 3,5\%

para las alternativas energéticas consideradas sin priorización de funciones.

Figura 6-29. Comparativa de los modelos PAMER, SEMA y SEPLAN para las alternativas energéticas consideradas. 


\section{LISTA DE TABLAS}

Tabla 3-1. Escala de Saaty. Método AHP. Fuente: Parodi (2013).............................. 35

Tabla 3-2. Clasificación de modelos de planificación energética (Domínguez, 2015). 46

Tabla 3-3. Modelos de planificación de demanda energética revisados en la presente tesis doctoral.

Tabla 3-4. Modelos de planificación de oferta energética revisados en la presente tesis doctoral.

Tabla 4-1. Resumen de las alternativas energéticas consideradas en el modelo SEPLAN.

Tabla 4-2. Niveles de comparación de la función objetivo $F_{1}$, descripción y evaluación numérica considerada. 78

Tabla 4-3. Coeficientes de electrificación en Perú por región (United Nations

Development Programme, 2009).

Tabla 4-4. Niveles de comparación de la función objetivo $F_{6}$, descripción y evaluación numérica considerada. 84

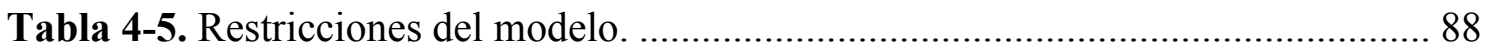

Tabla 4-6. Distancias normalizadas a ser minimizadas................................................ 89

Tabla 5-1. Características principales de la provincia de Cajamarca. 97

Tabla 5-2. Proyección de la demanda energética en la región de Cajamarca con los modelos sumativo y multiplicativo (ktep).

Tabla 5-3. Consumo de combustibles líquidos en la provincia de Cajamarca y su proyección al año 2025 .

Tabla 5-4. Demanda de las principales fuentes energéticas en la provincia de Cajamarca para el año 2025 en ktep.

Tabla 5-5. Energía sustituible por fuentes energéticas renovables para el año 2025 en ktep.

Tabla 5-6. Fuentes de información utilizadas para la estimación de la oferta de energías renovables en la provincia de Cajamarca.

Tabla 5-7. Evaluación de niveles de comparación para $\mathrm{F}_{1}$

Tabla 5-8. Cálculo de factores $\mathrm{M}$ y $\mathrm{N}$ para $\mathrm{F}_{1}$.

Tabla 5-9. Emisiones de ciclo de vida de $\mathrm{CO}_{2}$ de ER; $\mathrm{tCO}_{2} / \mathrm{tep}, \mathrm{tNO}_{\mathrm{x}} / \mathrm{tep}, \mathrm{tSO}_{2} /$ tep (Phen, 2006). 
Tabla 5-10. Evaluación de niveles de comparación para $\mathrm{F}_{6}$

Tabla 5-11. Cálculo de factores $\mathrm{M}$ y $\mathrm{N}$ para $\mathrm{F}_{6}$

Tabla 6-1. Establecimiento de escenarios según asignación de pesos a las funciones objetivo.

Tabla 6-2. Resultados obtenidos de la minimización de L $\infty$ para cada alternativa energética y considerando los escenarios estudiados (ktep).

Tabla 6-3. Priorización de funciones según los máximos valores alcanzados por las alternativas energéticas renovables. 


\section{CAPÍTULO I}

\section{INTRODUCCIÓN}

\subsection{Energía, desarrollo sostenible y acceso universal a la energía.}

La energía permite satisfacer las necesidades humanas más básicas: calor para la cocción de alimentos, agua caliente sanitaria y calefacción, iluminación y usos mecánicos (bombeo de agua, molido de alimentos, etc.). Además, es la base para la mayoría de las actividades económicas, ya sea para la producción de alimentos, el transporte y la comercialización, o simplemente para generar productos básicos para elaborar otros bienes o servicios de suministro (Rojas-Zerpa \& Yusta, 2014). En cualquier país en desarrollo, la energía representa un factor clave para alcanzar sus objetivos de crecimiento y apoyar la expansión de su economía (Kumar, et al., 2017).

El sector energético, desde la Revolución industrial hasta nuestros días, se ha fundamentado en el uso de combustibles fósiles. El carbón mineral y el petróleo han sido los principales recursos energéticos utilizados. Es la tendencia de la evolución del consumo energético desde 1776 en Estados Unidos (Figura 1-1), y aunque la información se refiere exclusivamente a este país, no deja de ser un reflejo de los cambios que ha habido globalmente a lo largo del tiempo. Aún hoy, a pesar de la irrupción de las energías renovables, las perspectivas de crecimiento de la demanda mundial de energía primaria parecen mantener al petróleo y al carbón como las principales fuentes energéticas (Figura $1-2)$.

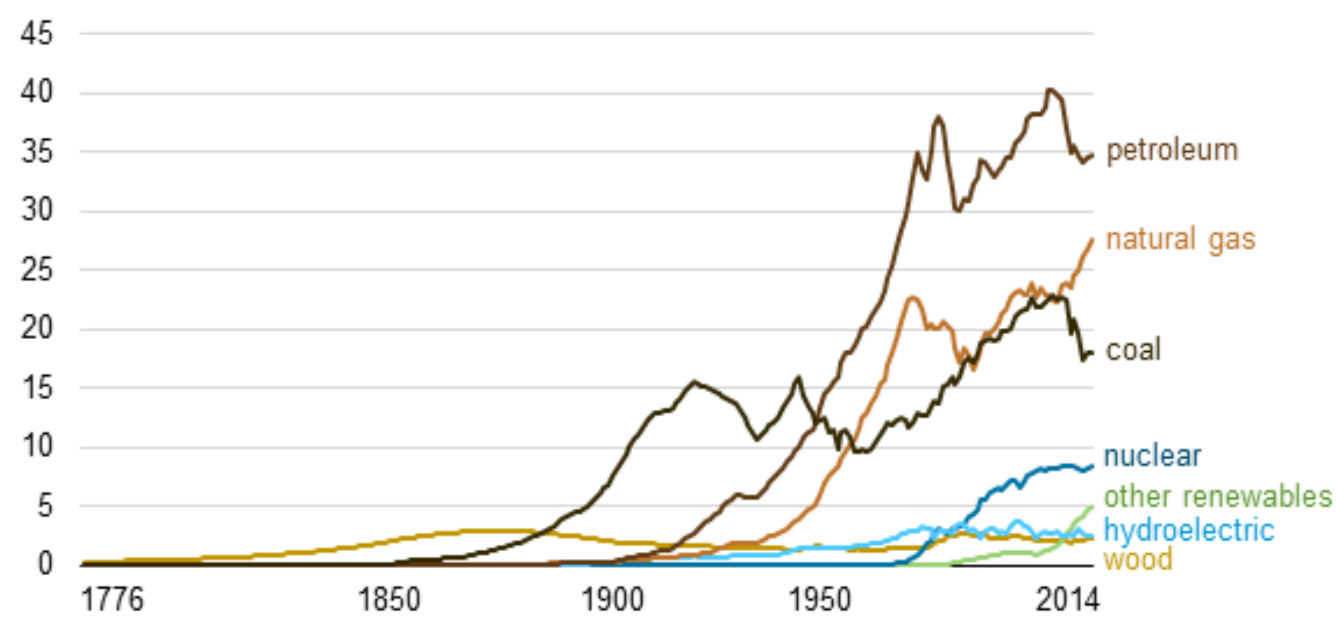

Figura 1-1. Consumo de energía en los Estados Unidos (1776-2014) en 10 15 BTU (U.S. Energy Information Administration, 2015) 


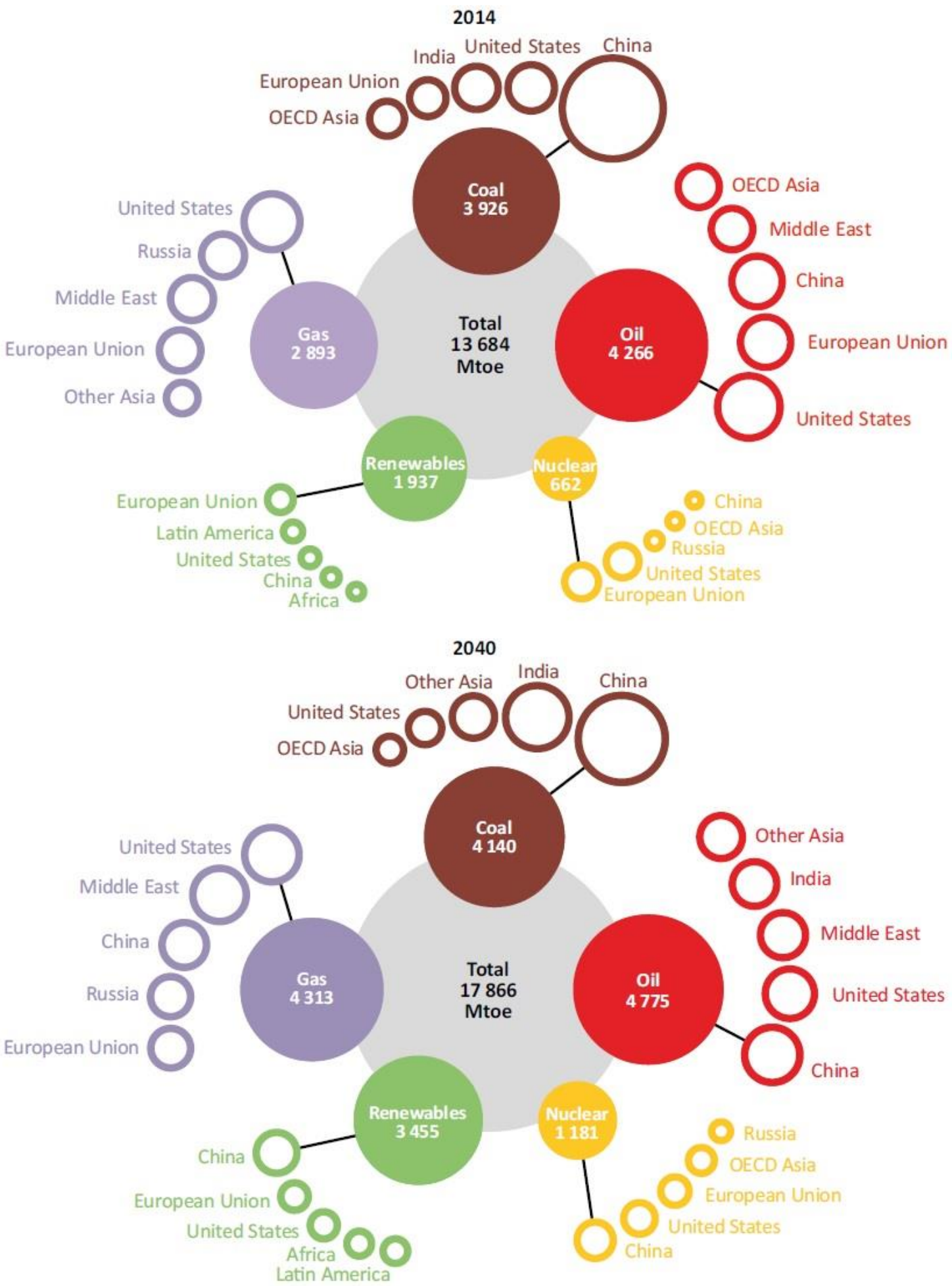

Figura 1-2. Perspectivas de crecimiento de la demanda mundial de energía primaria (International Energy Agency, 2016). 
Ante este panorama energético, diversos estudios comparten la conclusión de que el actual modelo energético mundial no es sostenible en términos económicos, sociales ni ambientales (Unsihuay-Vila, et al., 2011). El equilibrio de estos tres factores es fundamental para alcanzar lo que se conoce como desarrollo sostenible; es decir, para fomentar un desarrollo que no compromete el de las generaciones futuras. Según Roque (2015), el desarrollo sostenible se presenta como la intersección de tres desarrollos (Figura 1-3): el equitativo (económico y social), el vivible (social y medioambiental) y el viable (económico y medioambiental).

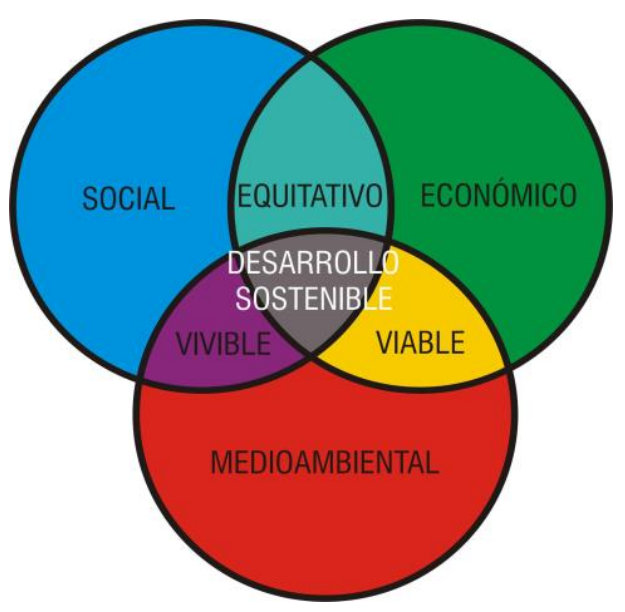

Figura 1-3. Desarrollo sostenible.

En este contexto, la promoción y fomento de fuentes alternativas o renovables de energía representa una apuesta por el desarrollo sostenible (Yadoo \& Cruickshank, 2012). El acceso a las fuentes renovables de energía permite resolver parcialmente todos los problemas generados por la utilización generalizada de combustibles fósiles: i) la dependencia energética de recursos no renovables, en muchos casos foráneos, y ii) un aumento elevado en la emisión de contaminantes (García, 2004). Dichos problemas han traído consigo una serie de retos y preocupaciones globales, como el cambio climático, el aumento de la demanda de energía y la seguridad energética. Ante tal situación, los mercados de energía renovable y los marcos normativos están evolucionando rápidamente por todo el mundo. Los gobiernos y los responsables políticos están introduciendo la legislación y los mecanismos de apoyo para acelerar el desarrollo del sector de las energías renovables, y muchos países tienen objetivos ambiciosos para la 
reducción de emisiones de $\mathrm{CO}_{2}$ (Troldborg, et al., 2014). Sin embargo, en el mundo, aún hay un nivel exiguo de acceso a energías limpias y renovables, y quedan por desarrollar tecnologías adecuadas de acumulación. Ha habido algunas inversiones en formas de producción y de transporte que consumen menos energía y requieren menos cantidad de materia prima, así como en formas de construcción o de saneamiento de edificios para mejorar su eficiencia energética, pero estas buenas prácticas están lejos de generalizarse (Francisco, 2015). Por su parte, los costes de las tecnologías renovables son cada vez más competitivos, especialmente en Latinoamérica. La Agencia Internacional de Energías Renovables (IRENA), en su estudio: Renewable Power Generation Costs in 2014 (IRENA, 2015), afirma que los costes de generación de algunas energías renovables son iguales o inferiores a los que se tienen con los combustibles fósiles tradicionales.

Esta aspiración de lograr la sostenibilidad energética se ha convertido en un reto de futuro internacional. La asamblea de las Naciones Unidas ha declarado la década 20142024 como la "Década de Energía Sostenible para todos", para garantizar el acceso a servicios energéticos asequibles, confiables, sostenibles y modernos para todos (Kumar, et al., 2017). Naciones Unidas articula el reto de energía sostenible mediante tres grandes metas: el acceso universal a la energía, aumentar el uso de energía renovable y mejorar la eficiencia energética (Naciones Unidas, 2015).

En efecto, en pleno siglo XXI, aproximadamente 1.200 millones de personas (17\% de la población mundial) vive sin electricidad y 2.700 millones de personas $(38 \%$ de la población mundial) pone en riesgo su salud a través del uso leñas para cocinar (International Energy Agency, 2016), siendo principalmente África y parte de Asia y Sudamérica, las zonas con mayor pobreza energética (Figura 1-4).

En Latinoamérica, uno de los esfuerzos por lograr el desarrollo sostenible se centra en el suministro de energía a las zonas rurales aisladas con nuevos modelos de suministro que garanticen la sostenibilidad y accesibilidad a la población de bajos ingresos (Organización Latinoamericana de Energía, 2013). 


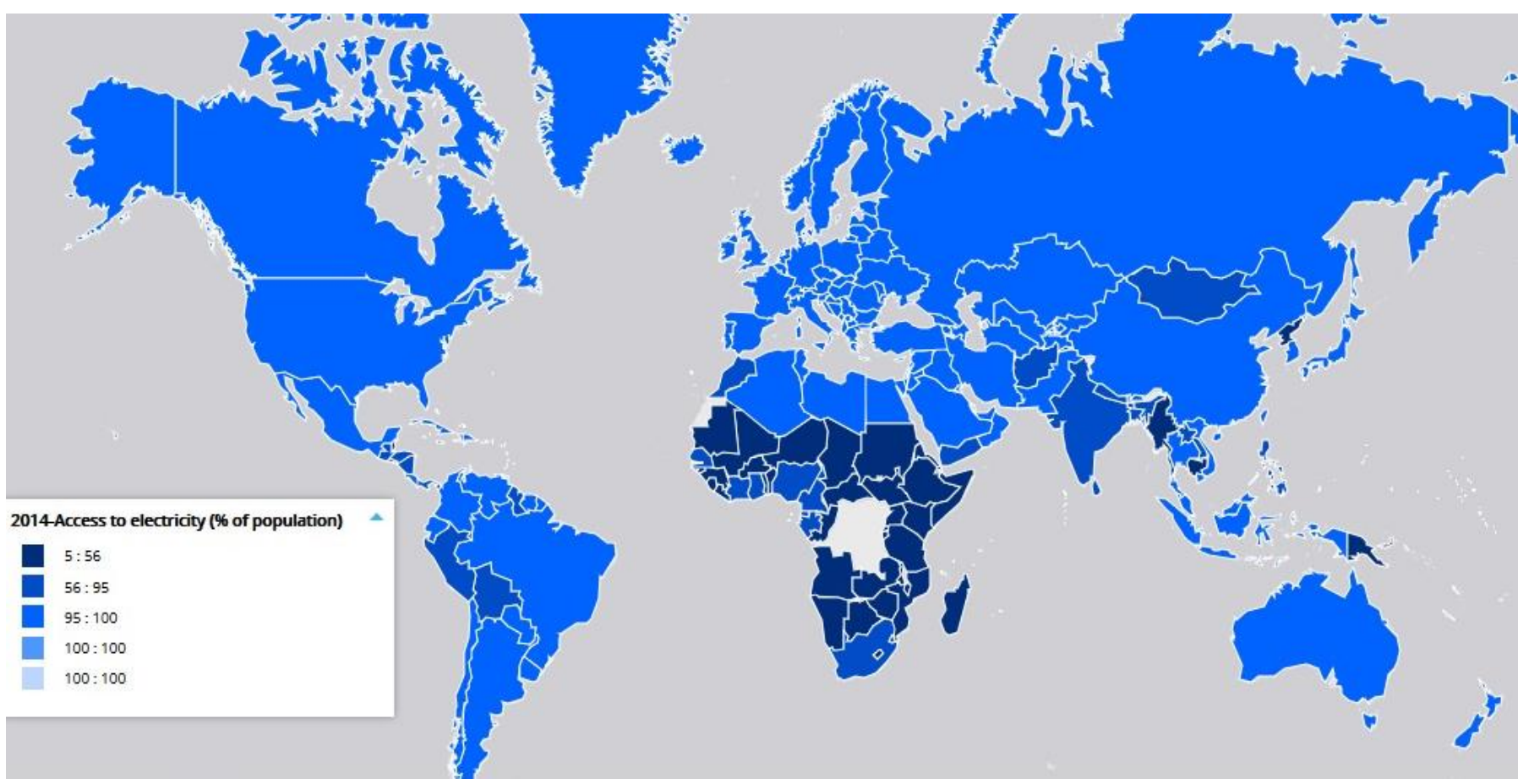

Figura 1- 4. Acceso universal a la electricidad al 2014 (\% de población) (World Bank, 2014).

En este sentido, la planificación energética se ha convertido en una herramienta de fundamental importancia para el desarrollo de los países y es considerada cada vez con mayor relevancia en sus hojas de ruta. Para alcanzar el desarrollo sostenible, las acciones deben ser tomadas en diferentes escalas temporales y espaciales, las cuales pueden considerarse dentro de la planificación energética (Huang, et al., 2015).

El objetivo de la planificación energética se centra en encontrar un conjunto de fuentes y mecanismos de conversión de energía a fin de satisfacer la demanda energética de una manera óptima (Kaya \& Kahraman, 2011). Para ello, los modelos de planificación energética evalúan y seleccionan entre las diferentes tecnologías energéticas, aquellas que atendiendo a la demanda energética actual y futura en la región, buscan satisfacerla de acuerdo a una serie de restricciones y factores de diversa índole (Mourmouris \& Potolias, 2013).

Durante la crisis del petróleo de la década de los 70, para abordar los problemas energéticos, eran populares los enfoques con un único criterio para determinar las opciones de suministro más eficientes a un bajo costo. Sin embargo, en la década de los 80, la creciente conciencia ambiental modificó dicho marco de decisiones (Kaya \& Kahraman, 2010). Surgió entonces la necesidad de incorporar consideraciones 
ambientales y sociales en la planificación energética. Tal como se ha desarrollado, actualmente el escenario de planificación energética tiene múltiples objetivos, definiciones y criterios. Un sistema de planificación adecuado debe considerar aspectos i) económicos (minimización de costes), ii) técnicos (ahorro y eficiencia energética, iii) ambientales (reducción de emisiones de gases de efecto invernadero y polución ambiental), iv) sociales (acceso universal a la energía), y v) políticos (seguridad y diversificación de suministro, independencia energética) (Unsihuay-Vila, et al., 2011).

Para resolver problemas tan complejos, la toma de decisiones basada en técnicas multicriterio ha demostrado ser una de las mejores herramientas para una planificación energética eficiente (Pohekar \& Ramachandran, 2004), (Kumar, et al., 2017), facilitando a los políticos y gestores, la toma de decisiones en temas energéticos conforme a sus preferencias, en casos donde hay más de un criterio en conflicto (Løken, 2007).

\subsection{La situación energética de Perú}

Perú es el segundo país de Sudamérica, después de Haití, con más población sin acceso a la electricidad. Según información oficial, en Perú hay 2,5 millones de personas sin acceso a la electricidad y 2,5 millones de hogares que utilizan combustibles tradicionales (Consorcio de Investigación Económica y Social, 2016). La tasa de cobertura eléctrica global del país es relativamente alta, sin embargo, el acceso a la electricidad en las zonas rurales sigue siendo una preocupación.

El acceso universal a la energía se enmarca en los compromisos recientemente contraídos por el gobierno de Perú, al refrendar en la reciente Asamblea General de las Naciones Unidas los denominados objetivos de desarrollo sostenible (Figura 1-5). Concretamente, el objetivo 7: "Garantizar el acceso a una energía asequible, segura, sostenible y moderna para todos" (Consorcio de Investigación Económica y Social, 2016). 

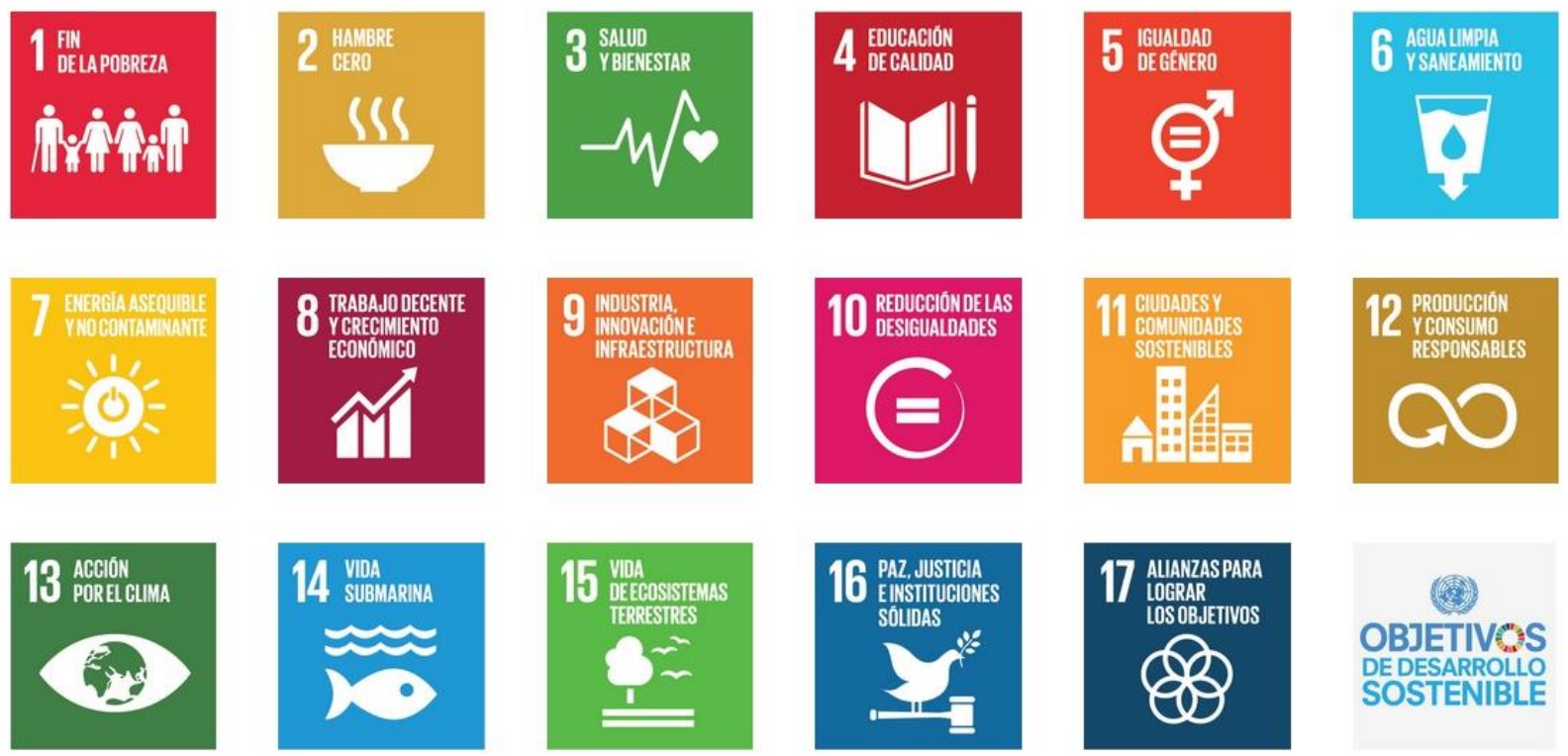

Figura 1-5. Objetivos de Desarrollo Sostenible (Naciones Unidas, 2015).

Otro hito importante para Perú fue la celebración de la COP20 $20^{1}$ en Lima en el 2014, que ha supuesto para Perú y para Latinoamérica un impulso para su compromiso en la reducción de emisiones de $\mathrm{CO}_{2}$ con el objetivo de frenar el calentamiento global. El acuerdo alcanzado sigue la línea del trabajo iniciado en la COP17 de Durban (Sudáfrica). El enfoque reflejado en Lima ha sido más global e independiente del desarrollo de cada país. A diferencia del Protocolo de Kioto, que implicaba solo a los “desarrollados", este acuerdo inclusivo engloba a todos los países.

Como resultado de la COP20 se firmó el acuerdo de Lima: "Lima Call for Climate Action", documento que recogió los principales compromisos de la conferencia, dentro de ellos se encuentra la financiación del Fondo Verde. Este fondo permitirá hacer llegar las diferentes tecnologías a los países en vías de desarrollo en su lucha contra el cambio climático, lo cual impulsará el desarrollo de las energías renovables.

Actualmente en Perú, el gas natural representa la principal fuente de energía primaria, con casi el $66 \%$ de participación en el mix energético. La segunda fuente más importante es el petróleo crudo con el 15,43 \% (Figura 1-6). Teniendo en cuenta que aún existe una importante dependencia energética por la importación de petróleo $(61 \%$ del

\footnotetext{
${ }^{1}$ La COP es la Conferencia de las Partes de la Convención Marco de las Naciones Unidas sobre el Cambio Climático (CMNUCC). La CMNUCC cuenta con 195 paí es firmantes. Se denomina "Partes" a los paíse miembros de la Convención. El Perú firmó la Convención en 1992, ratificándola al año siguiente. Entró en vigencia el 21 de marzo de 1994.
} 
total consumido en el $2014^{2}$ ), el gobierno peruano, además de impulsar la exploración y desarrollo de los hidrocarburos, promueve la eficiencia energética y el uso de energías renovables para la generación de energía eléctrica.

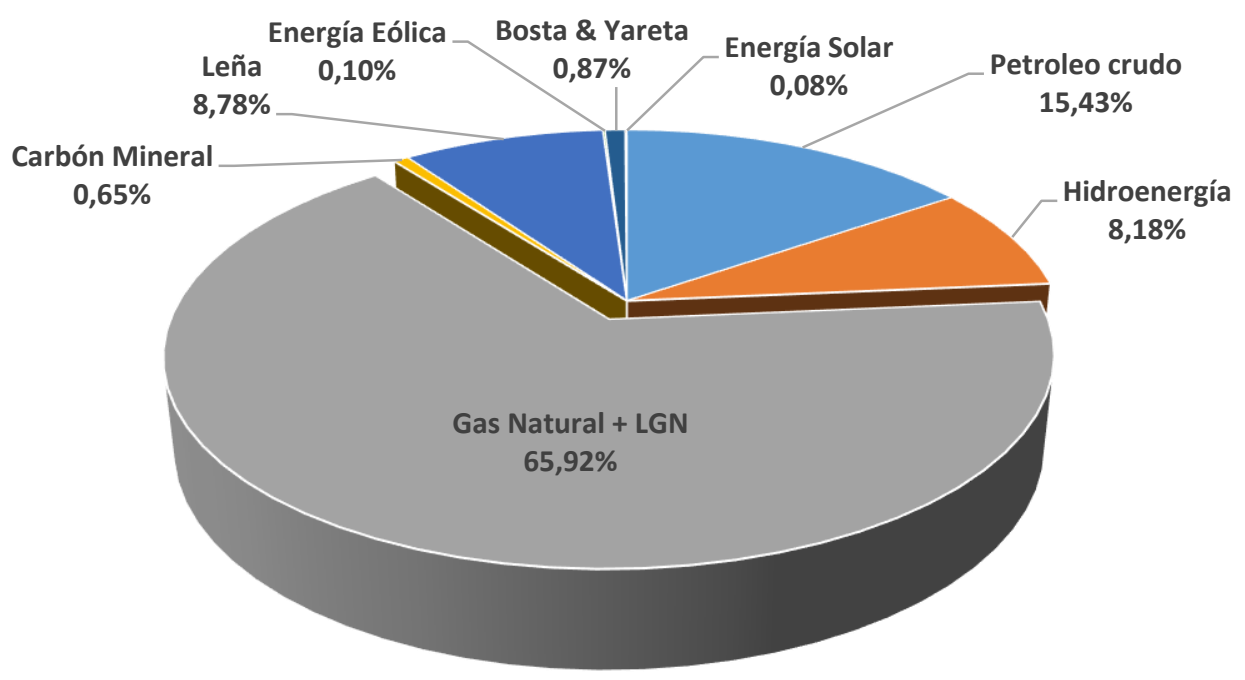

Figura 1-6. Consumo de Energía Primaria en Perú en el año 2014 (OSINERGMIN, 2015b).

Con respecto a la generación de energía eléctrica, en promedio, $48 \%$ de la electricidad generada proviene del agua y el 51\% de hidrocarburos (principalmente gas). Esta relación varía durante el año según la disponibilidad de agua en los embalses. Así, de diciembre a junio la generación hidroeléctrica es más elevada.

La Figura 1-7 muestra la situación de la generación de energía eléctrica para noviembre de 2016. En el día de máxima demanda (14 de noviembre), los hidrocarburos participaron con 53,1\%, las centrales hidroeléctricas con 41,0\%, la energía solar y eólica con $3,0 \%$, carbón con $1,7 \%$, diésel y residual con $0,9 \%$ y la biomasa y biogás con $0,3 \%$.

\footnotetext{
${ }^{2}$ (OSINERGMIN, 2015a)
} 


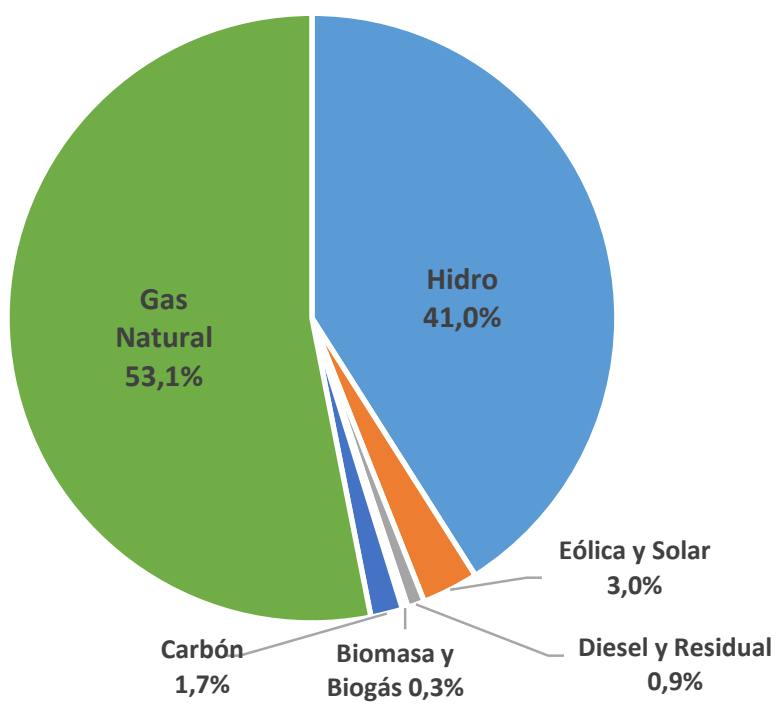

Figura 1-7. Fuentes energéticas para la generación de energía eléctrica en Perú. Día de máxima demanda. 14 de Noviembre, 2016 (Delta Volt, 2016).

Con la puesta en marcha de dos parques eólicos en septiembre 2014, la generación de energía de fuentes renovables no tradicionales superó por primera vez el $2 \%$ y en abril de 2016 alcanzó el 2.56\% gracias a la conexión del nuevo parque eólico "Tres Hermanas” en el departamento de Ica (Delta Volt, 2016).

Estas nuevas inversiones en el sector energía son consecuencia del importante crecimiento económico que el país ha experimentado en los últimos años. Según el Plan Energético Nacional 2014-2025, publicado por el Ministerio de Energía y Minas en noviembre de 2014, en el periodo 2003-2013, el PBI se incrementó un 86\% y la producción de electricidad aumentó un $92 \%$, en tanto que la producción de hidrocarburos lo hizo en un 260\%. En el mismo período, 2003-2013, el consumo final nacional de electricidad se incrementó en un 92\% mientras que el consumo de hidrocarburos líquidos y gas natural agregado creció un $100 \%$. Todo lo anterior representa el mayor crecimiento de la actividad económica y de la demanda de energía de las últimas décadas, gracias a la creciente inversión privada en infraestructura, así como por la inversión social desarrollada por el Estado (Ministerio de Energía y Minas, 2014). Asimismo, con respecto a las energías renovables, se indica: "La contribución de las energías renovables tanto en electricidad como en los hidrocarburos es una política de Estado, como una contribución desde este ámbito a la mitigación de los efectos del cambio climático. Habiéndose logrado que las energías renovables representen un $60 \%$ del mix de generación eléctrica en 2011”. 
Gracias a este crecimiento, Perú dispone de una tasa de cobertura global eléctrica relativamente elevada (92\%); si bien irregularmente repartida. Las áreas rurales y en menor medida en las zonas periurbanas, presentan importantes problemas de acceso eléctrico y a los combustibles modernos (75.2\%); con el agravante de que la falta de acceso energético afecta más negativamente a mujeres y niños por su rol en las viviendas, a los campesinos e indígenas que habitan mayoritariamente en entornos rurales, y a los jóvenes, obligándoles a emigrar en busca de oportunidades para su futuro (Consorcio de Investigación Económica y Social, 2016).

En la región de Cajamarca, ubicada en el norte del Perú, el 70\% de la población vive en zonas rurales. Por su parte, la dispersión de hogares en dichas zonas ha provocado que el porcentaje de acceso a la energía eléctrica en dicha región sea el más bajo del país (51\%) (Fondo Multilateral de Inversiones, 2014).

Otro importante problema del sector energético en Perú, ha sido la carencia de una estrategia y planificación dirigida. En el estudio "Evaluación del marco normativo e institucional del Perú para la promoción de energía eléctrica a partir de recursos renovables” publicado por el Banco Interamericano de Desarrollo en 2012 (Batlle, et al., 2012), se indica que: "Salvo casos puntuales, en el sector energía de Perú no existe un esquema de planificación formal y ordenada. El modelo de planificación vigente en Perú no contempla la elaboración de estudios estratégicos de medio plazo y/o de corto plazo que incluyan la indicación de los proyectos hidroeléctricos que se pueden desarrollar, la implementación de las metas de energías renovables no convencionales, la determinación del mix de generación hacia el futuro, etcétera".

En este contexto, esta situación representa una oportunidad muy interesante para introducir, a la planificación convencional, nuevos modelos que incorporen técnicas metodológicas adecuadas a la realidad concreta de una región o zona geográfica, y que tengan especial atención a las zonas rurales aisladas.

\subsection{Planificación energética en entornos rurales aislados. Técnicas multicriterio. Región de Cajamarca}

Las áreas rurales aisladas, especialmente en países en desarrollo, presentan mayor dificultad de acceso a la energía. En Latinoamérica y el Caribe (LAC) la situación de 
acceso a la energía puede parecer buena si se comparan tasas de acceso, pero aún existen entre 26 y 30 millones de personas que carecen de electricidad y unos 87 millones de personas sin combustible y/o modos de combustión modernos (Consorcio de Investigación Económica y Social, 2016). Pero pese a no ser grandes cantidades, comparadas con India o África subsahariana, las dificultades propias de falta de infraestructuras, dispersión geográfica y ubicaciones remotas, hace que llegar con redes convencionales a todas las viviendas resulte prácticamente imposible en un plazo razonable.

Por otro lado, el bajo consumo eléctrico per cápita de los usuarios rurales hace que la tarifa eléctrica no cubra los gastos de operación y mantenimiento de la ampliación de redes convencionales. Ante esta tesitura, una opción económicamente y técnicamente fiable para facilitar el acceso a los servicios eléctricos básicos a las comunidades rurales aisladas es la utilización de sistemas descentralizados con energías renovables (Real Academia de Ingeniería, 2011)

En los últimos años, se han desarrollado varios modelos de planificación energética para áreas rurales que incorporan energías renovables. Ferrer-Martí et al. propusieron el modelo MILP (Mixed integer linear programming) para optimizar el diseño de sistemas eólicos híbridos en una comunidad rural aislada (Ferrer-Martí, et al., 2013). HOMER (Hybrid Optimization Model for Multiple Energy Resources), programa desarrollado por el Laboratorio Nacional de Energía Renovable de los EE.UU. (NREL), propone un modelo de optimización para evaluar aplicaciones en energía distribuida para la generación autónoma en lugares remotos. Es una herramienta útil y ha sido aplicada en un número importante de países en desarrollo, obteniéndose buenos resultados. Sin embargo, HOMER, solamente obtiene resultados basados en dos criterios: económico y técnico. Ciertamente, estos son aspectos cruciales para cualquier sistema eléctrico fuera de la red, pero existen impactos significativos en el ámbito social y ambiental, criterios que HOMER no considera en su diseño (Cherni, et al., 2007).

ViPOR o similares, han logrado sistematizar el diseño y el dimensionamiento de los proyectos de planificación. Este tipo de software solo incorpora el análisis cuantitativo en un esquema de proyecto y se utiliza como una herramienta en la planificación. Sin embargo, según Nerini et al., una planificación energética adecuada en áreas rurales requiere una visión general más amplia y debe considerar los criterios técnicos, 
económicos, ambientales, sociales e institucionales en un enfoque de criterios múltiples (Nerini, et al., 2014).

La mayoría de las veces, incluir criterios ambientales y sociales, implica el uso de enfoques cualitativos (por ejemplo, el Método de las precedencias) para asignar pesos según las entrevistas a los expertos con el fin de clasificar los criterios, por ejemplo, Nerini et al. (2014) en la Amazonía brasileña, Amer \& Daim (2011) en Pakistán o Domínguez \& Marcos (2015) en Sri Lanka.

Rojas-Zerpa \& Yusta, en su revisión de metodologías y aplicaciones para el suministro eléctrico en áreas rurales remotas, encontraron que solo el $2 \%$ de los estudios se llevaron a cabo en Perú. Estos autores también concluyen que una planificación coherente y rigurosa en áreas rurales remotas, debe tomar en consideración las necesidades de la población local. Del mismo modo, algunas organizaciones no gubernamentales (ONG's) consideran que, en las comunidades rurales aisladas, las soluciones tecnológicas no son suficientes para garantizar la sostenibilidad de la planificación energética. Según la empresa de servicios sociales ACCIONA Microenergía Perú (AMP), los aspectos sociales y culturales de la población local son cuestiones clave a tener en cuenta para los modelos de toma de decisiones en estas áreas. Sin embargo, muy pocos modelos tienen en cuenta estos criterios, ya que conocer las necesidades de la población local no es fácil de obtener (Falcón-Roque, et al., 2017).

Sin embargo, diversos autores (Cherni et al., 2007; Henao et al., 2012) señalan que muchas instalaciones y modelos de planificación en áreas rurales han dejado de funcionar o son defectuosos por no considerar la opinión de la población local.

En este contexto, el objetivo del presente estudio es desarrollar un modelo de planificación energética utilizando herramientas de optimización multicriterio y que tome en cuenta a las comunidades rurales aisladas (CRA). Este modelo, llamado SEPLAN (Sustainable Energy Planning), incluye una función innovadora para evaluar la viabilidad de las diferentes tecnologías de energía renovable para un suministro sostenible de energía en las CRA, aplicando las restricciones de precedencia. El modelo SEPLAN también contiene otras funciones objetivo: i) sustitución de energía fósil por energías renovables; ii) emisiones evitadas de gases de efecto invernadero (GEI); y iii) la función de costos. Dichas funciones fueron obtenidas en base a los estudios realizados por 
Domínguez \& Marcos (2015), Benítez (2015), García (2004) y Marcos (1985) en la Catedra de Termodinámica y Motores de la ETSI de Montes de Madrid.

La presente tesis doctoral busca aportar un nuevo enfoque al problema muchas veces complejo de la planificación energética de una región. En este sentido, el enfoque multicriterio ofrece métodos que pueden proporcionar alternativas de solución a los problemas inherentes a la gestión de la energía (Pohekar \& Ramachandran, 2004). 


\section{CAPÍTULO II}

\section{OBJETIVOS}

\subsection{Objetivo General}

- Elaborar un modelo de planificación energética con energías renovables empleando técnicas de optimización multicriterio y que tenga en cuenta el acceso universal a la energía en las CRA.

\subsection{Objetivos Específicos}

- Validar el modelo propuesto aplicándolo a la provincia de Cajamarca - Perú.

- Evaluar el estado de las energías renovables en la provincia de Cajamarca - Perú y su uso potencial para la generación de energía eléctrica, térmica y para su uso en el transporte.

- Establecer la priorización de las alternativas tecnológicas renovables en la planificación energética de la zona de estudio, según los criterios considerados.

- Evaluar el establecimiento de escenarios por asignación de pesos a los criterios considerados, para representar la opinión de los centros decisores.

- Realizar un análisis de sensibilidad de los resultados obtenidos.

- Comparar los resultados obtenidos por el modelo propuesto con los modelos PAMER y SEMA, que han sido utilizados como base para su desarrollo.

- Incorporar al modelo propuesto el objetivo del acceso universal a la energía en las CRA, además de considerar aspectos sociales, económicos y medioambientales. 


\section{CAPÍTULO III}

\section{ESTADO DEL ARTE}

Tal como se definió en el Capítulo I de la presente tesis doctoral, la planificación energética tiene como objeto identificar el conjunto de fuentes y mecanismos de conversión de energía que satisfaga de una manera óptima la demanda energética en un ámbito territorial (Kaya \& Kahraman, 2011). Asimismo, se ha indicado que, si se pretende que dicha planificación sea sostenible, se requiere atender a múltiples criterios y objetivos en ocasiones contradictorios: criterios económicos, sociales, ambientales, etc. Es por este motivo que, para el desarrollo de modelos de planificación con dichas características, se imponen las técnicas de análisis de decisión multicriterio.

En la aplicación de técnicas multicriterio se busca realizar una toma de decisiones en base a todas las soluciones posibles, que son aquellas que satisfacen las restricciones del problema. Estas decisiones posibles se ordenan con arreglo a un cierto criterio que representa las preferencias del centro decisor. Esta función de criterio recibe el nombre de función objetivo. Recurriendo a técnicas matemáticas relativamente sofisticadas, se establece la "solución óptima", como aquella solución factible para la que la función objetivo alcanza un valor óptimo (Romero, 1996).

Según Wang et al., en la revisión que realizan sobre métodos de análisis de decisión multicriterio, se indica que dicho análisis es una forma de evaluación de sostenibilidad integrada. Asimismo, que es un enfoque de evaluación operacional y apoyo a las decisiones que es adecuado para abordar problemas complejos con alta incertidumbre, objetivos contradictorios, diferentes formas de datos e información, múltiples intereses y perspectivas, y la contabilidad de sistemas biofísicos y socioeconómicos complejos y en evolución. Los métodos pueden proporcionar soluciones que permitan aumentar la complejidad de los problemas de gestión de energía (Wang, et al., 2009).

En general, el problema del análisis de decisión multicriterio para la toma de decisiones en una planificación energética sostenible implica considerar $m$ alternativas evaluadas según $n$ criterios (Wang, et al., 2009). Según esto, la matriz de decisión agrupada se puede expresar de la siguiente manera: 


$\left.\begin{array}{ccccc}\text { Criterio } & C_{1} & C_{2} & \cdots & C_{n} \\ (\text { Pesos } & w_{1} & w_{2} & \cdots & w_{n}\end{array}\right)$

Alternativas

$$
X=\begin{gathered}
A_{1} \\
A_{2} \\
\vdots \\
A_{m}
\end{gathered} \quad\left[\begin{array}{cccc}
x_{11} & x_{12} & \cdots & x_{1 n} \\
x_{21} & x_{22} & \cdots & x_{2 n} \\
\vdots & \vdots & \ddots & \vdots \\
x_{m 1} & x_{m 2} & \cdots & x_{m n}
\end{array}\right]_{m x n}
$$

Donde $x_{i j}$ es el valor del criterio j-ésimo de la i-ésima alternativa, $w_{j}$ es el peso del criterio $\mathrm{j}, n$ es el número de criterios y $m$ es el número de alternativas.

La resolución de esta ecuación, según Wang et al., sería uno de los últimos pasos dentro del proceso del análisis de decisión multicriterio. Las cuatro etapas que se mencionan para llevar a cabo dicho proceso son las siguientes: formulación de alternativas y selección de criterios, ponderación de criterios, evaluación y tratamiento final y agregación. El paso preliminar en el análisis de decisión multicriterio es formular las alternativas para el problema a partir de un conjunto de criterios seleccionados y para normalizar los datos originales de los criterios. En segundo lugar, se determinan los pesos de los criterios para mostrar su importancia relativa en el análisis. Luego, las alternativas aceptables se clasifican por métodos de análisis de decisión multicriterio con ponderaciones de criterios. Finalmente, el ranking de alternativas es ordenado. Si el orden de clasificación de las alternativas con diferentes métodos es el mismo, el proceso ha terminado. De lo contrario, los resultados de clasificación se agregan nuevamente y se selecciona el mejor esquema.

A continuación, se describen algunos métodos multicriterio y los modelos de planificación energética que se han considerado más relevantes para la presente tesis doctoral, muchos de ellos desarrollados en base a dichos métodos.

\subsection{Métodos multicriterio usados en planificación energética.}

En el estudio realizado por Pohekar \& Ramachandran (2004), donde se hace una revisión de más de 90 artículos publicados, se indica que los métodos multicriterio más aplicados en planificación energética fueron la optimización multiobjetivo, AHP y ELECTRE. Las áreas de aplicación de los métodos multicriterio en dichos trabajos fueron la planificación de energías renovables, la asignación de recursos energéticos, la gestión de energía de edificios, la gestión de energía de transporte, la planificación de proyectos 
de energía, la planificación de servicios eléctricos y otras áreas diversas. A continuación, se describen los métodos mencionados y otros de interés.

\subsubsection{Programación Multiobjetivo.}

Los modelos multiobjetivo permiten a los responsables de la toma de decisiones considerar la naturaleza conflictiva y los compromisos entre objetivos diferentes, para seleccionar soluciones satisfactorias para el problema de planificación energética. Se han propuesto modelos de programación multiobjetivo potentes y atractivos con el fin de incorporar de manera efectiva las consideraciones ambientales, por ejemplo, en la planificación de la expansión de la generación eléctrica (Unsihuay-Vila, et al., 2011).

Para Romero (1993) la programación multiobjetivo constituye un enfoque multicriterio de gran potencialidad cuando el contexto decisional está definido por una serie de objetivos a optimizar que deben de satisfacer un determinado conjunto de restricciones. Como la optimización simultánea de todos los objetivos es usualmente imposible, el enfoque multiobjetivo en vez de intentar determinar un no existente óptimo pretende establecer el conjunto de soluciones eficientes o Pareto óptimas.

Planteado el problema en estos términos, la estructura general de un programa multiobjetivo puede representarse esquemáticamente de la siguiente manera:

$$
\operatorname{Eff} f(x)=\left[f_{1}(x), \ldots f_{i}(x), \ldots f_{n}(x)\right]
$$

Sujeto a:

$$
X \epsilon F
$$

E $f f=$ Significa la búsqueda de soluciones eficientes o Pareto óptimas.

fi $(x)=$ Expresión matemática del atributo i-ésimo.

$\mathrm{x}=$ Vector de variables de decisión.

$\mathrm{F}=$ Conjunto de restricciones -usualmente lineales- que definen el conjunto de soluciones posibles.

\subsubsection{La matriz de pagos en la programación multiobjetivo}

La matriz de pagos es un mecanismo de gran utilidad, pues permite cuantificar el nivel de conflicto existente entre los objetivos que estamos analizando. La mecánica 
operativa para obtener la matriz de pagos puede resumirse de la siguiente manera: se optimiza cada objetivo separadamente calculándose seguidamente los valores alcanzados por los demás objetivos en cada solución óptima (Romero, 1993).

\subsubsection{Método de las restricciones}

Marglin (1967) demostró que, si en un problema multiobjetivo uno de los objetivos se optimiza mientras que los demás objetivos se incorporan al conjunto de las restricciones como restricciones paramétricas, se genera un punto eficiente para cada conjunto de valores asignados al vector de términos independientes. Si tenemos q objetivos a maximizar, el método de las restricciones da lugar al siguiente problema:

$$
\operatorname{Maximizar} \mathrm{f}_{\mathrm{k}}(\mathrm{x})
$$

Sujeto a:

$$
\begin{gathered}
X \in F \\
\mathrm{f}_{\mathrm{j}}(\mathrm{x}) \geq \mathrm{L}_{\mathrm{j}} \quad \mathrm{j}=1,2, \ldots, \mathrm{k}-1, \mathrm{k}+1, \ldots, \mathrm{q}
\end{gathered}
$$

Donde F representa el conjunto de soluciones posibles. Por medio de variaciones paramétricas de términos independientes $\mathrm{L}_{\mathrm{j}}$ iremos generando el conjunto eficiente. Debe observarse que, si entre los objetivos situados como restricciones alguno de ellos debe minimizarse en vez de maximizarse, en tal caso, el sentido de la restricción paramétrica cambia.

El método de las restricciones garantiza la generación de soluciones eficientes sólo cuando las restricciones paramétricas son activas en el óptimo. Por el contrario, si en el óptimo alguna de las restricciones paramétricas no es activa y además existen óptimos alternativos, entonces la solución generada por este método puede no ser eficiente. Esta situación, aunque factible teóricamente, no es en absoluto usual que se presente en la práctica, por lo que el método no pierde su carácter de potente generador de puntos eficientes (Romero, 1993).

\subsubsection{Método de las ponderaciones}

Zadeh (1963) demostró que, si a cada objetivo se le asocia un peso no negativo, y seguidamente, se procede a la agregación de todos los objetivos, la optimización de dicha función agregada y ponderada genera para cada conjunto de pesos un punto extremo eficiente. Así, para un problema decisional con q objetivos a maximizar, la aplicación de este método conduce al siguiente programa matemático de tipo paramétrico: 
Sujeto a:

$$
\text { Maximizar } \mathrm{W}_{1} \mathrm{f}_{1}(\mathrm{x})+\mathrm{W}_{2} \mathrm{f}_{2}+\ldots+\mathrm{W}_{\mathrm{q}} \mathrm{f}_{\mathrm{q}}(\mathrm{x})
$$

$$
\begin{aligned}
& X \epsilon F \\
& W \geq 0
\end{aligned}
$$

Para cada vector de pesos $\mathrm{W}$ se obtiene un punto extremo eficiente. Por tanto, variando paramétricamente los pesos, se puede generar, el conjunto eficiente. Debe observarse que el método de las ponderaciones, a diferencia del método de las restricciones, no genera puntos interiores sino sólo puntos extremos eficientes (Romero, 1993).

\subsubsection{Programación Compromiso.}

Unsihuay-Vila et al. (2011) propone un modelo multiescalar, multiobjetivo y multirriesgo para integrar la planificación de la expansión, a largo plazo, de la generación y transporte de energía de los sistemas eléctricos. Dicho modelo, de nombre MESEDES, incluye tres objetivos contradictorios para generar un conjunto de alternativas de expansión: a) La minimización de los costes de inversión y operativos; b) reducción al mínimo de las emisiones de GEI de las centrales eléctricas; y c) la maximización de la seguridad del suministro basada en la diversidad de los recursos primarios, incluidas las importaciones de energía. Para la resolución del problema se utilizó la Programación Compromiso.

La Programación Compromiso consiste en utilizar un punto ideal como un punto de referencia para el centro decisor. Como en el punto ideal cada objetivo alcanza su valor óptimo, parece lógico aceptar que un comportamiento racional por parte del centro decisor consistirá en elegir aquel punto eficiente o zona del conjunto eficiente que se encuentre más próxima al punto ideal. Para poder medir la mayor o menor proximidad de un punto eficiente con respecto al punto ideal, se introduce en el análisis una función de distancia. Es importante observar que en este enfoque el concepto de distancia no se utiliza sólo en un sentido geométrico, sino más bien como un subrogado de las preferencias humanas (Romero, 1993).

\subsubsection{Determinación de las soluciones compromiso}

La programación compromiso se apoya en el "Axioma de Zeleny" que puede enunciarse de la siguiente manera: 
"Dadas dos soluciones posibles en el espacio de los objetivos $\mathrm{f}_{1} \mathrm{y}_{2}$ la solución preferida será aquella que se encuentre más próxima al punto ideal” (Zeleny, 1973). Este axioma puede formularse de la siguiente manera:

$$
\begin{aligned}
& f_{1} \mathrm{P} f_{2} \Leftrightarrow \mathrm{D}\left(f_{1}\right)<\mathrm{D}\left(f_{2}\right) \\
& f_{2} \mathrm{P} f_{1} \Leftrightarrow \mathrm{D}\left(f_{2}\right)<\mathrm{D}\left(f_{1}\right) \\
& f_{1} \mathrm{I} f_{2} \Leftrightarrow \mathrm{D}\left(f_{1}\right)=\mathrm{D}\left(f_{2}\right)
\end{aligned}
$$

donde $\mathrm{P}$ e I son las tradicionales relaciones de preferencia e indiferencia y D ( $f$ i) representa la distancia (según una determinada métrica) existente entre la solución fi y el correspondiente punto ideal.

Dado el usual grado de conflicto entre objetivos, el punto ideal es inalcanzable, por lo que se hace necesario buscar soluciones compromiso. Para ello, se debe definir el grado de proximidad dj entre el objetivo j-ésimo y su ideal como:

$$
d_{j}=\left[f_{j}^{*}-f_{j}(x)\right]
$$

Lo siguiente es agregar los grados de proximidad para todos los objetivos del problema decisional. Ahora bien, hay que tener en cuenta que usualmente los objetivos están medidos en unidades distintas, por lo que la suma de los grados de proximidad no tiene sentido o significado al carecer de homogeneidad dimensional. Por tanto, habrá que proceder a la normalización de los objetivos. Una posible forma de normalizar los objetivos, obteniendo grados de proximidad homogéneos, es la siguiente:

$$
d_{j}=\frac{\left[f_{j}^{*}-f_{j}(x)\right]}{\left[f_{j}^{*}-f_{* j}\right]}
$$

Donde $d_{j}$ representa el grado de proximidad del objetivo $j$-ésimo normalizado y $f_{*_{j}}$ es el anti ideal de dicho objetivo. El grado de proximidad normalizado está acotado entre 0 y 1 . Así, cuando un objetivo alcanza su valor ideal, su grado de proximidad es cero; por el contrario, dicho grado se hace igual a uno cuando el objetivo en cuestión alcanza un valor igual al anti ideal. Si representamos ahora por $\mathrm{W}_{\mathrm{j}}$ las preferencias que el centro decisor asocia a la discrepancia existente entre la realización del objetivo j-ésimo y su ideal, la programación compromiso se convierte en el siguiente problema de optimización: 


$$
\operatorname{Min} L_{p}=\left[\sum_{j=1}^{n} W_{j}^{p}\left|\frac{f_{j}^{*}-f_{j}(x)}{f_{j}^{*}-f_{* j}}\right|^{p}\right]^{1 / p}
$$

Sujeto a:

$$
x \in F
$$

Las barras de valor absoluto de este modelo pueden eliminarse, pues $f_{j}^{*} \geq f_{j}(x)$ para cualquier objetivo, por tratarse $\mathrm{f}^{*}{ }_{\mathrm{j}}$ de una componente del vector ideal. Para la métrica $\mathrm{p}=1$, la mejor solución compromiso o punto más próximo al ideal se puede obtener resolviendo el siguiente programa lineal:

$$
\operatorname{Min} L_{1}=\sum_{j=1}^{n} W_{j} \frac{f_{j}^{*}-f_{j}(x)}{f_{j}^{*}-f_{* j}}
$$

Sujeto a:

$$
x \in F
$$

Para métrica $\mathrm{p}=\infty$, se minimiza la máxima desviación de entre todas las desviaciones individuales; esto es, para $p=\infty$ sólo la desviación mayor influye en el proceso de minimización. Para esta métrica, la mejor solución compromiso se puede obtener resolviendo el siguiente programa lineal:

$$
\begin{array}{cc}
\text { Sujeto a: } & \text { Min } \mathrm{L}_{\infty}=\mathrm{d} \\
x \in F & \\
W_{1} \frac{f_{1}^{*}-f_{1}(x)}{f_{1}^{*}-f_{* 1}} \leq d \ldots \ldots W_{i} \frac{f_{i}^{*}-f_{i}(x)}{f_{i}^{*}-f_{* i}} \leq d \ldots \ldots W_{q} \frac{f_{q}^{*}-f_{q}(x)}{f_{q}^{*}-f_{* q}} \leq d
\end{array}
$$

Donde d representa la desviación más grande.

\subsubsection{El teorema de Yu}

Para obtener la mejor solución compromiso para métricas distintas de $\mathrm{p}=1 \mathrm{y} p=$ $\infty$, se hace necesario recurrir a algoritmos de programación matemática no lineal. Así, se deduce que para $\mathrm{p}=2$ la solución compromiso más próxima al punto ideal se obtendrá resolviendo el siguiente programa no lineal: 


$$
\operatorname{Min} L_{2}=\sum_{j=1}^{n} W_{j}^{2}\left[\frac{f_{j}^{*}-f_{j}(x)}{f_{j}^{*}-f_{* j}}\right]^{2}
$$

La dificultad de determinar el mejor conjunto compromiso a partir de este programa no lineal queda considerablemente reducida si tenemos en cuenta lo descubierto por $\mathrm{Yu}$ (1973). Este autor demostró que, para problemas de dos objetivos, los puntos $\mathrm{L}_{1}$ y $\mathrm{L}_{\infty}$ definen un subconjunto de la frontera eficiente denominado por Zeleny (1974) conjunto compromiso. Las otras mejores soluciones compromiso pertenecen al conjunto acotado por dichos puntos $\mathrm{L}_{1}$ y $\mathrm{L}_{\infty}$.

Posteriormente, Freimer \& Yu (1976) demostraron que, para problemas con más de dos objetivos, los puntos $\mathrm{L}_{1}$ y $\mathrm{L}_{\infty}$ no tienen que definir necesariamente un conjunto compromiso. Es decir, en contextos de más de dos objetivos para métricas distintas de $\mathrm{p}$ $=1 \mathrm{y} \mathrm{p}=\infty$, pueden existir soluciones compromiso que no pertenezcan al intervalo cerrado $\left[\mathrm{L}_{1}, \mathrm{~L}_{\infty}\right]$. Aunque esta posibilidad es cierta, sin embargo, es poco probable que se presente en la práctica, por lo que la potencialidad de la programación compromiso se mantiene al no ser necesario usualmente computar modelos complicados de programación matemática no lineal.

\subsubsection{Programación por metas.}

Una aplicación práctica de este método la realiza Ching-Ter (2014), quien, basado en el modelo Ramón \& Cristóbal (Ramón \& Cristóbal, 2012), propone un nuevo modelo de programación por metas para abordar el problema de localización de cinco centrales energéticas renovables para la generación de energía en cinco localidades de la Comunidad Autónoma de Cantabria, en el norte de España. Por otro lado, Silva \& Nakata (2009), emplean el método para evaluar opciones de electrificación rural en zonas rurales aisladas de Colombia.

La optimización multiobjetivo y la programación compromiso constituyen instrumentos muy potentes para analizar problemas decisionales, sin embargo, la utilidad de los mismos se reduce considerablemente en problemas decisionales de un tamaño relativamente elevado. Así, si estamos analizando un problema con muchos atributos y con un conjunto de restricciones relativamente complejo, tal tipo de problema puede resultar computacionalmente intratable. Por ello, es conveniente que dichos enfoques den 
paso a otros con una solidez teórica tal vez menor, pero con una operatividad muy superior. Dentro de esta línea pragmática puede encuadrarse la programación por metas (Romero, 1993).

La programación por metas se apoya en una lógica no optimizante sino en lo que Herbert Simon $(1955,1957)$ ha acuñado como lógica satisfaciente. El premio Nobel Simon conjetura que, en las complejas organizaciones actuales, el contexto decisional está definido por información incompleta, recursos limitados, multiplicidad de objetivos, conflictos de intereses, etc. Simon conjetura que en este tipo de contexto el centro decisor más que optimizar una o varias funciones objetivo intenta que una serie de metas relevantes se aproximen lo más posible a unos niveles de aspiración fijados de antemano (Romero, 1993).

\subsubsection{Estructura general de un modelo de programación por metas}

En primer lugar, se deben fijar los atributos que consideremos relevantes para el problema analizado. Una vez establecidos los atributos, se asignan a cada uno de ellos un nivel de aspiración. Se entiende por nivel de aspiración el nivel de logro que el centro decisor desea alcanzar. Seguidamente, se conecta el atributo con el nivel de aspiración, por medio de la introducción de las variables de desviación negativa y positiva, respectivamente. Así, para el atributo i-ésimo, tendremos la siguiente meta:

$$
\mathrm{f}_{\mathrm{i}}(\mathrm{x})+\mathrm{n}_{\mathrm{i}}-\mathrm{p}_{\mathrm{i}}=\mathrm{t}_{\mathrm{i}}
$$

donde, $\mathrm{f}_{\mathrm{i}}(\mathrm{x})$ representa la expresión matemática del atributo i-ésimo, $\mathrm{t}_{\mathrm{i}}$, el nivel de aspiración asociado a dicho atributo, $\mathrm{n}_{\mathrm{i}} \mathrm{y} \mathrm{p}_{\mathrm{i}}$ las variables de desviación negativa y positiva respectivamente. La variable de desviación negativa cuantifica la falta de logro de una meta con respecto a su nivel de aspiración, mientras que la variable de desviación positiva cuantifica el exceso de logro de una meta con respecto a su nivel de aspiración.

Una variable de desviación es no deseada cuando al decidor le conviene minimizarla para alcanzar el objetivo planteado. Pueden distinguirse tres casos:

- Si el objetivo debe maximizarse, la variable no deseada es $\mathrm{n}_{\mathrm{i}}$.

- Si el objetivo debe minimizarse, la variable no deseada es $p_{i}$.

- Si se desea alcanzar exactamente el nivel de aspiración del objetivo, ambas variables de desviación son no deseadas. 
El proceso de minimización de las variables de desviación no deseadas puede ejecutarse de forma diferente (Romero, 1996). En la programación por metas ponderadas se engloban convenientemente ponderadas todas las variables de desviación, en una sola función objetivo. Algebraicamente, el modelo puede expresarse como:

$$
\operatorname{Min} \sum_{1}^{n}(\alpha i n i+\beta i p i)
$$

sujeto a

$$
\begin{aligned}
& \mathrm{f}_{\mathrm{i}}(\mathrm{x})+\mathrm{n}_{\mathrm{i}}-\mathrm{p}_{\mathrm{i}}=\mathrm{t}_{\mathrm{i}} \\
& \mathrm{x} \in \mathrm{R} \\
& \mathrm{x} \geq 0 \quad \mathrm{n}_{\mathrm{i}} \geq 0 \quad \mathrm{p}_{\mathrm{i}} \geq 0 \quad \text { para } \mathrm{i}=1,2, \ldots \mathrm{n}
\end{aligned}
$$

donde $\alpha_{i}$ y $\beta_{\mathrm{i}}$ son los pesos asociados a las variables de decisión negativa y positiva respectivamente y $\mathrm{R}$ es el conjunto de restricciones que definen el conjunto de soluciones posibles. Por otra parte, $\mathrm{n}_{\mathrm{i}} \mathrm{y} \mathrm{p}_{\mathrm{i}}$ deben expresarse en unidades homogéneas para lo cual puede trabajarse con desviaciones porcentuales dividiendo el valor en términos absolutos de la desviación entre su respectivo nivel de aspiración.

Para Belton y Stewart (2002), un área de incertidumbre al aplicar la programación por metas es la fijación de los niveles de aspiración por parte de los participantes. Las soluciones del problema son sensibles a las metas fijadas que pueden oscilar entre valores muy optimistas, no realistas y valores muy bajos. Los mejores resultados se obtienen cuando las metas seleccionadas son optimistas pero posibles.

Por otra parte, el método requiere que los objetivos estén asociados a atributos cuantificables. De esta forma, el método se limita a problemas que puedan ser formulados en esos términos y excluye problemas que incorporan criterios que se evalúan subjetivamente. En este último caso, la programación por metas puede usarse como una fase previa al análisis multicriterio propiamente dicho, para generar una lista más corta de alternativas que luego pueden ser analizadas de acuerdo a los aspectos no cuantificables (Belton \& Stewart, 2002).

\subsubsection{Proceso de Jerarquías Analíticas}

El estudio de Pohekar (2003), concluye que el Proceso de Jerarquías Analíticas, AHP por sus siglas en inglés (Analytical Hierarchy Process), es el método más usado en la planificación energética sostenible. Kaya (2010) hace uso de este método integrándola 
con VIKOR (Método de toma de decisiones multiatributo) para determinar la mejor alternativa de energía renovable para Estambul. Asimismo, realiza una selección entre los sitios de producción de energía alternativa en esta ciudad utilizando el mismo enfoque. En la metodología VIKOR-AHP propuesta, los pesos de los criterios de selección se determinan mediante matrices de comparación de AHP.

El Proceso de Jerarquías Analíticas es una metodología de apoyo a la decisión multicriterio que propone la evaluación y posterior ordenamiento de las alternativas de acción planteadas mediante la estructuración de un árbol jerárquico donde el nivel superior es ocupado por el objetivo fundamental en términos de su enunciado y el segundo nivel corresponde a los aspectos generales o criterios más importantes relevantes a la evaluación; subdividido cada uno de ellos en los criterios y subcriterios que se juzgue conveniente incluir para definir completamente el problema. Las alternativas de solución planteadas ocupan el último nivel.

La Figura 3-1 muestra un árbol jerárquico de cinco niveles donde el segundo nivel está ocupado por " $n$ " criterios cada uno de los cuales es subdividido en subcriterios de acuerdo al grado de detalle que se desee. El último nivel es ocupado por "s" alternativas (Parodi, 2013).

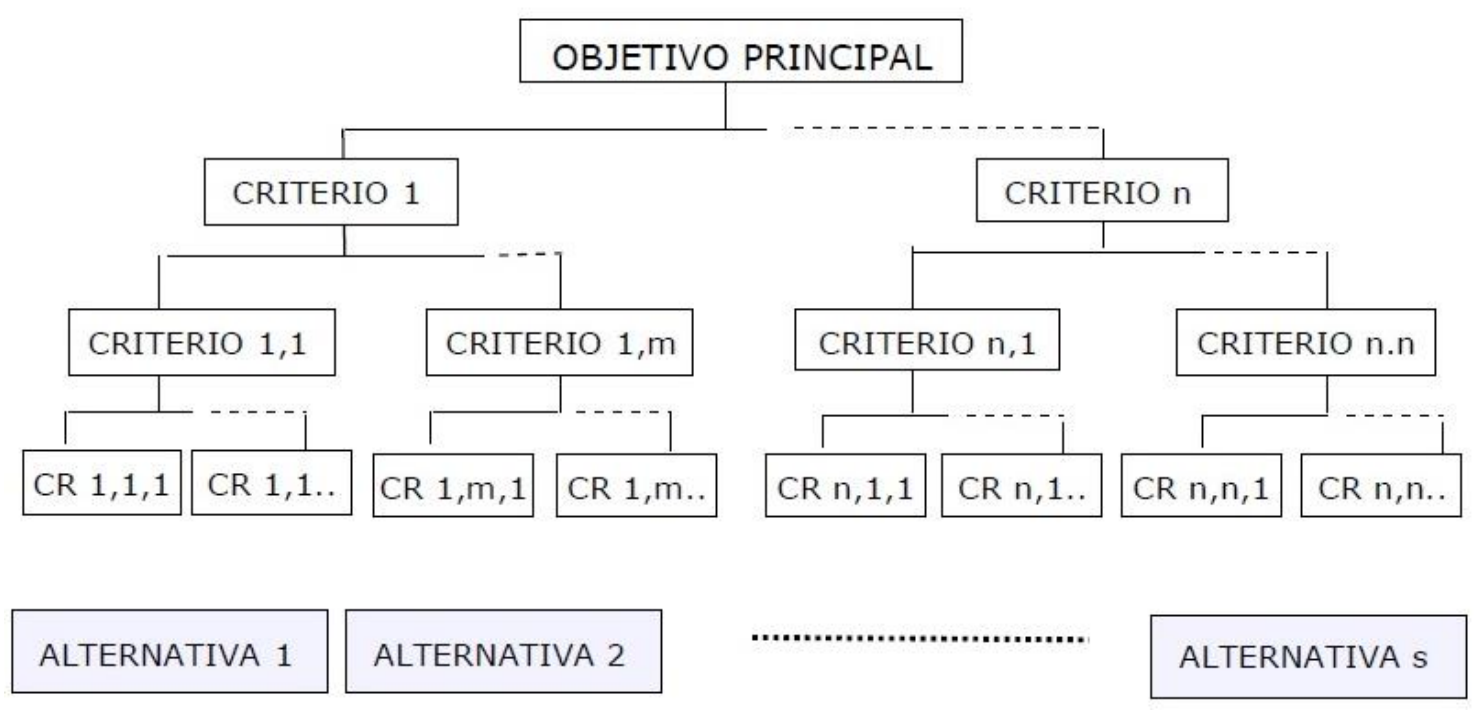

Figura 3- 1. Modelo de Jerarquías Analíticas (Parodi, 2013).

Una vez que la jerarquía ha sido estructurada deben establecerse las prioridades locales o importancia relativa de cada criterio con relación al nivel jerárquicamente superior. Las comparaciones se hacen por pares, entre los criterios del mismo nivel (o 
entre las alternativas en el último nivel) cuyo peso se intenta determinar; preguntándose en cada caso, cuánto más importante es el criterio i que el criterio j, con relación al criterio superior al que están asociados. Las comparaciones se inician en el nivel de las alternativas, se continúan con el nivel inmediatamente superior; y así sucesivamente hasta alcanzar el nivel más alto. La Tabla 3-1 muestra la escala fundamental que utiliza el AHP para las comparaciones por pares (Saaty, 1986).

Tabla 3-1. Escala de Saaty. Método AHP. Fuente: Parodi (2013).

\begin{tabular}{|c|l|l|}
\hline wi/wj & \multicolumn{1}{|c|}{ DEFINICIÓN } & \multicolumn{1}{|c|}{ EXPLICACIÓN } \\
\hline 1 & Igual & $\begin{array}{l}\text { Ambos criterios se consideran } \\
\text { igualmente importantes. }\end{array}$ \\
\hline 3 & Moderada & $\begin{array}{l}\text { El criterio i es ligeramente más } \\
\text { importante que el criterio j. }\end{array}$ \\
\hline 5 & Fuerte & $\begin{array}{l}\text { El criterio i es fuertemente más } \\
\text { importante que el criterio j. }\end{array}$ \\
\hline 7 & Muy fuerte o demostrada & $\begin{array}{l}\text { El criterio i es mucho más } \\
\text { importante que el criterio j. }\end{array}$ \\
\hline 9 & Extrema & $\begin{array}{l}\text { El criterio i más } \\
\text { incuestionablemente } \\
\text { importante que el criterio j. }\end{array}$ \\
\hline $2,4,6,8$ & Valores intermedios & Valores intermedios \\
\hline
\end{tabular}

Cada nivel en la escala de Saaty corresponde a la intensidad (wi/wj) de la importancia del criterio i sobre el criterio j, con relación al criterio superior. En caso de que el criterio j sea más importante que el criterio i, el valor a asignar es el inverso.

Los valores $(\mathrm{aij}=\mathrm{wi} / \mathrm{wj})$ obtenidos a través de las opiniones individuales expresadas por cada participante en el proceso, se acomodan en matrices de juicios; una para cada ronda de comparaciones binarias (Saaty, 2005a). Si el decisor es consistente se genera una matriz [A] cuyas entradas, aij, satisfacen las siguientes condiciones (Saaty, 2005b):

(i) $\mathrm{Si}$ aij $=$ a entonces $\mathrm{aji}=1 / \mathrm{a}$

(ii) $\mathrm{Si} \mathrm{j}=\mathrm{i}$ entonces aij $=\mathrm{aji}=\mathrm{aii}=1$ para todo $\mathrm{i}$. 
La matriz recíproca $[\mathrm{A}]$ cumple con la igualdad: $[\mathrm{A}][\mathrm{w}]=\mathrm{n}[\mathrm{w}]$, donde $[\mathrm{w}]$ es el vector de prioridades o pesos de los criterios [w1, w2 ... wn] y n la dimensión de la matriz; esto es,

$$
\left[\begin{array}{cccc}
w 1 / w 1 & w 1 / w 2 & \ldots \ldots & w 1 / w n \\
w 2 / w 1 & w 2 / w 2 & \ldots \ldots & w 2 / w n \\
\ldots \ldots & \ldots \ldots & & \ldots \ldots \\
w n / w 1 & w n / w 2 & \ldots \ldots & w n / w n
\end{array}\right]\left[\begin{array}{c}
w 1 \\
w 2 \\
\ldots \ldots \\
w n
\end{array}\right]=n\left[\begin{array}{c}
w 1 \\
w 2 \\
\ldots \ldots \\
w n
\end{array}\right]
$$

Si el decisor es no consistente se genera una matriz [R] que es una perturbación de $[\mathrm{A}]$ y que cumple con la igualdad: $[\mathrm{R}][\mathrm{w}]=\lambda \max [\mathrm{w}]$, donde $\lambda \max$ es el autovalor dominante de la matriz de comparación y [w] su autovector. Puede demostrarse que $\lambda \max$ $>$ n, donde la igualdad significa consistencia perfecta (Shtub, et al., 1994).

Finalmente, se define el índice de consistencia, $\mathrm{IC}=(\lambda \max -\mathrm{n}) /(\mathrm{n}-1)$ y la relación de consistencia, RC = IC /RI; donde RI es un promedio de los índices de consistencia de una gran muestra de matrices cuyas entradas se escogen de forma aleatoria. Saaty (1986) obtuvo los siguientes resultados para RI,

\begin{tabular}{|c|cccccccccc|}
\hline $\mathrm{n}$ & 1 & 2 & 3 & 4 & 5 & 6 & 7 & 8 & 9 & 10 \\
\hline $\mathrm{RI}$ & 0,00 & 0,00 & 0,58 & 0,90 & 1,12 & 1,24 & 1,32 & 1,41 & 1,45 & 1,49 \\
\hline
\end{tabular}

La experiencia de Saaty sugiere que la relación de consistencia debe ser menor a 0,1 para que los resultados sean confiables.

Finalmente, el peso global asociado a cada criterio i (o alternativa, en el último nivel) se obtiene multiplicando su peso local wi por el peso global del criterio superior al que está relacionado. La suma de los pesos globales de cada alternativa con respecto a cada criterio proporciona la evaluación de cada alternativa.

Cuando el análisis se realiza en grupo deben combinarse los juicios de los participantes de forma que se cumpla la condición de reciprocidad; esto es, que se cumpla que el valor síntesis de los recíprocos de los juicios individuales sea igual al recíproco del valor síntesis de esos juicios; esto es, para un grupo de n participantes donde xi es el juicio del participante $\mathrm{i}$,

$$
F(1 / x 1,1 / x 2, \ldots 1 / x n)=1 / F(x 1 . x 2, . . x n)
$$


Se ha comprobado que sólo la media geométrica de los valores satisface la condición anterior. De esta forma, en AHP para obtener el resultado consolidado para el grupo se toma la media geométrica de las prioridades globales obtenidas de las jerarquías individuales de cada individuo participante (Parodi, 2013).

\subsubsection{Método ELECTRE}

El método ELECTRE es uno de los métodos multicriterio concebidos específicamente para analizar problemas decisionales discretos, y el de mayor aplicación. Fue desarrollado por Roy (1968). Desde entonces, tanto las aplicaciones como los desarrollos teóricos de este método han sido muy intensos.

Mourmouris y Potolias (2013), desarrollaron un método llamado REGIME, con características similares al ELECTRE, para la planificación energética con energías renovables y aplicándolo al caso concreto de Thassos, Grecia. El REGIME también es un método discreto que permite comparar un conjunto finito de alternativas. La información cualitativa es transformada en cuantitativa con el fin de ser analizada más fácilmente.

El método ELECTRE consiste en un procedimiento para reducir el tamaño del conjunto de soluciones eficientes. Tal reducción se realiza por medio de una partición del conjunto eficiente en un subconjunto de alternativas más favorables para el centro decisor (el núcleo) y en otro subconjunto de alternativas menos favorables. Para abordar tal tarea, se introduce el concepto de "relación de sobreclasificación" que es consustancial al ELECTRE en todas sus variantes (Romero, 1996).

Una elección o alternativa $E_{i}$ sobreclasifica a otra alternativa $E_{k}$ cuando para los atributos considerados, el enunciado "la alternativa $E_{i}$ es al menos tan buena como la alternativa $E_{k}$ ” es válido. La sobreclasificación se establece en base a dos conceptos: concordancia y discordancia. La concordancia cuantifica hasta qué punto para un elevado número de atributos $\mathrm{E}_{\mathrm{i}}$ es preferida a $\mathrm{E}_{\mathrm{k}}$. Por otra parte, la discordancia cuantifica hasta qué punto no existe ningún atributo para el que $\mathrm{E}_{\mathrm{k}}$ es mucho mejor que $\mathrm{E}_{\mathrm{i}}$.

Para que la alternativa $E_{i}$ sobreclasifique a la alternativa $E_{k}$, y por tanto forme parte del núcleo o subconjunto de alternativas más favorables, es necesario que la concordancia entre $E_{i}$ y $E_{k}$ supere un umbral mínimo establecido y que, asimismo, la discordancia entre $E_{i}$ y $E_{k}$ no supere otro umbral también establecido de antemano. Cuando esto sucede, puede decirse que la alternativa $E_{i}$ es preferida a la alternativa $E_{k}$ 
desde casi cualquier punto de vista, aunque ello no implique que $\mathrm{E}_{\mathrm{i}}$ domine, desde un punto de vista paretiano, a $E_{k}$.

La principal ventaja de la relación de sobreclasificación es que en ella no subyace necesariamente el supuesto de transitividad de preferencias o de comparabilidad, que sí subyace a cualquier enfoque basado en funciones de utilidad. Así, si $E_{1} \mathrm{~S}_{2}$ y $\mathrm{E}_{2} \mathrm{~S} \mathrm{E}_{3}$ (donde S representa la relación de sobreclasificación) esto no implica necesariamente que $\mathrm{E}_{1} \mathrm{~S}_{3}$. Así el ELECTRE reconoce con acierto que las razones que llevan al centro decisor a preferir $E_{1}$ a $E_{2} y$ aquellas que le llevan a preferir $E_{2}$ a $E_{3}$ pueden ser muy diferentes y no conducir, por tanto, a que $E_{1}$ sea preferida a $E_{3}$. En cuanto a la comparabilidad en muchos contextos decisionales, frecuentemente como sucede en la ingeniería de sistemas, el centro decisor no puede o no desea comparar alternativas debido, entre otras posibles razones, a falta de información, insuficiente precisión en las mediciones, inconmensurabilidad de los criterios, etc.

La relación de sobreclasificación se utiliza para formar un grafo en el que cada vértice del mismo representa una de las alternativas o elecciones no dominadas. A partir de este grafo, se establece un subgrafo, que constituye el núcleo del conjunto de alternativas favorables. Los vértices del núcleo representan las alternativas o elecciones que son preferidas según la relación de sobreclasificación establecida en base a los índices de concordancia y discordancia. Los vértices (alternativas) que no forman parte del núcleo se eliminan del análisis (Romero, 1996).

\subsubsection{Técnicas de análisis de información (Técnicas blandas).}

Las técnicas blandas comprenden una serie de técnicas de análisis de información vectorial, cuyo uso está muy difundido en estudios del medio físico, muy distintas a los algoritmos matemáticos mencionados en los apartados anteriores.

Estas técnicas fueron usadas por Marcos (1984), en su modelo de oferta de energías renovables, denominado PAMER, donde los coeficientes de las variables de la función objetivo fueron obtenidos mediante el método de las precedencias, método que se usa también para la determinación de dos de las funciones objetivo de la presente tesis doctoral.

Las técnicas de análisis vectorial son aplicables al tratamiento de información, cuando se pretende simplificar el proceso de jerarquización de vectores. Para esta 
jerarquización se consideran los vectores n-dimensionales, que aparecen al combinar una serie de funciones $f_{1}(x), f_{2}(x), \ldots f_{n}(x)$.

Según Otero (1979) hay tres posibilidades para procesar información:

- Transformación del vector $\mathrm{P}_{\mathrm{i}}$ en un escalar $\mathrm{p}_{\mathrm{i}}$ : Siendo una forma la atribución de pesos $\mathrm{W}$, a los elementos, y de valores $\mathrm{V}$, a los tipos de cada elemento (empleo de escalas cardinales):

$$
P_{i}=\sum_{j=1}^{n} W_{j} V_{i j}
$$

- Ordenación de vectores: Establecimiento de una relación de orden entre 2 vectores cualesquiera. Exige la relación previa de los tipos dentro de cada elemento con respecto a un objetivo determinado (empleo de escalas ordinales).

- Establecimiento de relaciones de semejanza o distinción con vistas a una determinada finalidad, entre los tipos en que se divide cada elemento, y a partir de ellas, cálculo de la "distancia" entre dos vectores (empleo de escalas nominales).

Las denominadas "técnicas blandas" forman un grupo de técnicas multidimensionales, que simplifican el número de decisiones a tomar por el decisor y no incurren en las complejidades conceptuales de la función de valor (Martínez-Falero \& González-Alonso, 1995). Según Holmes (1972), "las operaciones aritméticas son inapropiadas al tratar con datos o criterios no cuantificables". Este mismo autor propone un método para evaluar alternativas con tres pasos:

1. Ordenar la importancia de los criterios de elección u objetivos.

2. Ordenar las alternativas.

3. La alternativa colocada en primer lugar para el criterio más importante será la elegida.

Pero la validez de su aplicación, así como de las demás técnicas basadas en estas teorías, depende mucho del nivel de conocimientos que el decisor tenga, sobre aquello donde se va a aplicar, como un medio natural, usos energéticos, etc. (García, 2004).

Nijkamp (1980) define como "modelos de técnicas blandas" (soft models), a aquellos modelos capaces de seleccionar la mejor alternativa, en función de los valores que toman para los objetivos considerados en el proceso de decisión. Sólo se pide que los 
valores que tomen las alternativas para los distintos objetivos sean ordinales. Pero como hace notar Otero (1979), "el empleo de escalas ordinales tropieza con el arraigo de la cuantificación convencional que no admite matices ni tolera la imprecisión". En este sentido entre los puntos aducidos por algunos autores se encuentran, la mayor exactitud de los resultados en métodos cuantitativos, carentes de ambigüedades, frente a la mejor descripción del sentir general y las necesidades de las personas en los métodos ordinales.

En las técnicas blandas el tratamiento de información se reduce a elegir el vector que mejor satisfaga una serie de objetivos. Dado que en muchas ocasiones el decisor no puede comparar fácilmente las alternativas, puede ser útil buscar métodos de comparación menos exigentes que faciliten esta actividad (García, 2004).

Una aplicación concreta de estas técnicas la realiza Nerini (2014) para evaluar las opciones de electrificación rural en la amazonia brasileña. En el análisis se agregan criterios con una ponderación simple para obtener valores únicos para los criterios macro, y los que a su vez reciben pesos para llegar al índice final del análisis. Esta metodología permite la comparación de alternativas tanto en criterios únicos (por ejemplo, el coste del combustible) como en los criterios macro (por ejemplo, economía), al tiempo que muestra un índice agregado ponderado final (Nerini, et al., 2014).

A continuación, se describen algunos métodos que permiten la comparación entre distintas alternativas, basados en "técnicas blandas".

\subsubsection{Método de cascadas.}

Este método fue desarrollado para su uso en estimaciones de impactos (Newkirk, 1979). Su aplicación consiste en la elevación de varios valores de orden inferior a valores agregados más altos, al tiempo que garantiza que el valor agregado es mayor o igual al máximo. Para realizar esta promoción a un nivel superior se utilizan como elementos de comparación, el nivel y la base de la cascada. El nivel indica el valor a partir del cual se tienen en cuenta los valores a agregar. La base indica el número de valores iguales que deben producirse para realizar la promoción (García, 2004).

A cada variable le asignamos un vector. Por ejemplo, si tenemos cuatro factores cuyos vectores asociados son:

$\mathrm{V}_{1}=$ vector asociado al factor $1, \mathrm{~V}_{1}=(3,2,1,2)$

$\mathrm{V}_{2}=$ vector asociado al factor $2, \mathrm{~V}_{2}=(3,1,2,3)$ 
$\mathrm{V}_{3}=$ vector asociado al factor $3, \mathrm{~V}_{3}=(2,2,2,2)$

$\mathrm{V}_{4}=$ vector asociado al factor $4, \mathrm{~V}_{4}=(1,3,1,2)$

Estos vectores están compuestos por cuatro componentes, pero pueden tener todas aquellas que se establezcan para definir los factores.

Cuando existen dos componentes de igual valor en un mismo vector, se produce otra componente de valor superior, en una unidad. Las variables buscadas son aquellas que presentan la componente más alta. A esa componente del sector i se la denomina $\mathrm{c}\left(\mathrm{V}_{\mathrm{i}}\right)$.
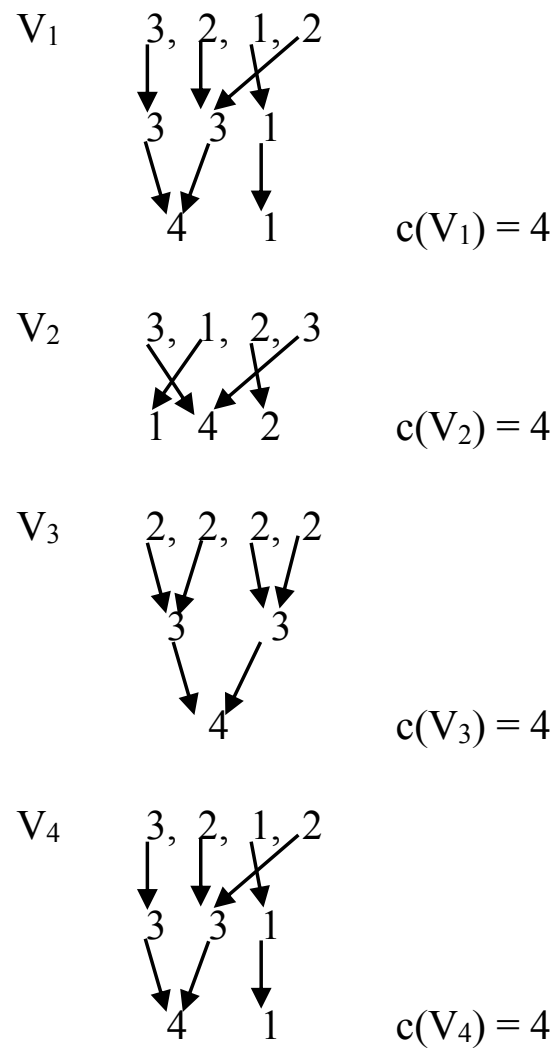

Como los valores $\mathrm{c}\left(\mathrm{V}_{\mathrm{i}}\right)$ son iguales, debemos ver los valores residuales y aplicar la comparación entre ellos; sea este valor residual c'.

$$
\begin{aligned}
& c^{\prime}\left(V_{1}\right)=1 \\
& c^{\prime}\left(V_{2}\right)=2,1 \\
& c^{\prime}\left(V_{3}\right)=\text { NULO } \\
& c^{\prime}\left(V_{4}\right)=1
\end{aligned}
$$


Conclusión:

$1^{\circ}$ En principio las cuatro variables deben ser consideradas.

$2^{\circ} \mathrm{El}$ orden de prioridad, relativa, ha de ser:

- Variable 2.

- Variable 1.

- $\quad$ Variable 4.

- Variable 3.

\subsubsection{Método del producto de componentes.}

Dado el vector $\mathrm{V}_{\mathrm{i}}\left(\mathrm{a}_{1}, \mathrm{a}_{2}, \mathrm{a}_{3}, \mathrm{a}_{4}\right)$ se define el número $\mathrm{P}_{\mathrm{i}}$ asociado al vector $\mathrm{V}_{\mathrm{i}}$ como:

$$
P_{i}=\prod_{j=1}^{n} a_{i j}
$$

El orden prioritario de variables es el derivado de un valor más alto en su número asociado P.

La información que este método es útil si todos los criterios, aplicados en las distintas componentes de los vectores, son de igual importancia. En muchas ocasiones no se cumple este requisito y el método pierde efectividad (García, 2004).

\subsubsection{Método de las precedencias.}

Otero (1979) desarrolla el método de las precedencias inversiones en su tesis doctoral. En el mismo compara cada alternativa con todas y cada una de las mismas, que se encuentran dentro del conjunto de decisión. La autora afirma que "la principal ventaja que presenta el método de las precedencias es, el facilitar la ordenación de las unidades que integran el territorio, sin recurrir a condicionamientos ajenos a su propia estructura". Esto es aplicable a otros campos como la energía. El modo de ordenar los distintos factores (componentes del vector que refleja una alternativa) se basa en el número de factores que precede, así como el número de factores por los que es precedido (García, 2004). 
Martínez-Falero \& González-Alonso (1995), explican que si el decisor puede establecer una ordenación de la importancia de los objetivos se introduce el concepto de inversiones. Los objetivos son enumerados según la serie natural, en función de su importancia. Los vectores con mayor importancia serán aquellos cuyos resultados formen una serie creciente de números, con independencia de cuáles sean éstos. Un índice, que determina el grado de preferencia es, el número de inversiones que deben realizarse para alcanzar el orden de los números naturales.

Estas dos clasificaciones se reflejan sobre dos ejes, uno para cada una, ordenándose los vectores según los puntos proyectados por los ejes.

Matemáticamente, el método de las precedencias tiene el siguiente proceso operativo. Cada variable $i$ está definida por un vector $V_{i}\left(a_{i 1}, a_{i 2}, \ldots a_{\text {in }}\right)$. A cada una se le asigna un nuevo vector de dos componentes, la primera indica el número de variables a las que precede y la segunda el número de variables por las que es precedida.

La primera componente se obtiene multiplicando las componentes del vector original:

$$
\prod_{j=1}^{n} a_{i j}
$$

La segunda componente se calcula mediante el producto de los complementos de las componentes del vector original hasta el máximo valor que puedan tomar $\left(\mathrm{N}_{\mathrm{i}}\right)$.

$$
\prod_{j=1}^{n}\left(N_{i}-a_{i j}\right)
$$

Un vector es estrictamente superior a otro si su primera componente es mayor y su segunda componente es menor (García, 2004).

\subsubsection{Método de la distancia al punto ideal.}

Desarrollado por Janardan y Schaiffer (1975), trata de establecer una norma que exprese una situación media o umbral, de modo que todo objetivo que supere esta situación implique un valor añadido.

El procedimiento consiste, en primer lugar, en ordenar los grados de consecución de los distintos objetivos. Una vez ordenados se transforman todos los valores iguales, o 
"valores a la norma", según un número que indique el valor medio de los valores superiores. Finalmente se define un índice de ordenación de las alternativas de la siguiente forma:

$$
V_{i}=\sqrt{\frac{A_{i}}{A_{i}+B_{i}}}
$$

donde:

$$
\begin{gathered}
A_{i}=\sum_{j=1}^{n}\left(\frac{r_{i j}-r_{e j}}{S_{j}}\right)^{2} \\
B_{i}=\sum_{j=1}^{n} r_{i j}
\end{gathered}
$$

$r_{i j}=$ Valor que toma la alternativa i, en el objetivo j, después de la segunda transformación.

$r_{e j}=$ Valor que toma la norma del objetivo j después de la segunda transformación.

$\mathrm{S}_{\mathrm{j}}=$ Desviación típica del objetivo j después de la primera transformación.

Las ventajas de utilizar este método son de dos tipos. Por un lado, permite emitir juicios relativos a cada alternativa, basándonos en el valor del índice $\mathrm{V}_{\mathrm{i}}$. Por otro, la variable ordenada: $\left(\mathrm{r}_{\mathrm{ij}}-\mathrm{r}_{\mathrm{ej}}\right) / \mathrm{S}_{\mathrm{j}}$ sigue una distribución normal, que no podría presentarse en datos cuantitativos (García, 2004).

\subsubsection{Programación no lineal.}

La programación no lineal es un caso particular de los problemas de optimización. En este tipo de problemas las restricciones y/o la función objetivo son no lineales.

La teoría básica para la programación no lineal se encuentra en el desarrollo de las condiciones Kuhn-Tucker. Estas son las condiciones necesarias para identificar puntos estacionarios en la programación no lineal. Su construcción se basa en una extensión del método del Lagragiano (García, 2004).

En la programación no lineal se representa con más fidelidad, las circunstancias en las que se desenvuelve la actividad económica, ya que normalmente se dispone de cantidades limitadas de recursos, pero sin la obligación de emplearlas en su totalidad, si ello no resulta necesario. De este modo, posibilita obtener soluciones óptimas que no 
saturen necesariamente todas las restricciones, pudiendo quedar recursos que no sea necesario utilizar hasta su agotamiento (Sánchez, 2013).

\subsection{Modelos de planificación energética}

Marcos (1984) ofrece una definición muy descriptiva de los modelos energéticos: "si fuéramos capaces de realizar una foto al proceso energético de la región, indicando el flujo de las distintas energías; su inicio, desde donde se extraen o compran, hasta el punto donde se consumen, indicando todas y cada una de las transformaciones sufridas; su transporte y distribución, señalando rendimientos y pérdidas, así como los residuos provocados en el proceso, esa foto sería un modelo energético".

El modelo en sí, no es más que una herramienta o un instrumento. Lo que importa es la visión de la realidad que podemos obtener de él. Los modelos energéticos tienen su desarrollo después de la primera crisis del petróleo. En 1976 la Agencia Internacional de la Energía (AIE) constituye un grupo de trabajo con la misión de analizar los sistemas energéticos de los países que la integran. Este hecho supuso el desarrollo del modelo de oferta energética MARKAL (Market Allocation). Los pioneros en la realización de modelos energéticos son técnicos de empresas dedicadas a la venta de energía, como las grandes empresas petrolíferas. Los primeros desarrollos datan de 1870 y se sitúan en EEUU. Los modelos que hoy utilizan las grandes compañías petrolíferas (como EXXON, MOBIL, SHELL, BP, TEXACO, etc.) son el desarrollo de aquéllos, unidos a la incorporación de las nuevas técnicas de cálculo por ordenador mucho más rápidas y complejas que las que se podían usar en el siglo XIX (García, 2004).

Existen muchas formas de hacer una caracterización o clasificación de los modelos existentes $\mathrm{y}$, como en todo, la clasificación última sería resaltar las particularidades individuales de cada modelo. También existen las clasificaciones definidas a partir de las condiciones que parecen relevantes a determinadas instituciones, como es el caso de la clasificación de modelos energéticos que utiliza el Banco Mundial (Álvarez, 2004).

Domínguez (2015) propone una clasificación de acuerdo a unos criterios de diferenciación, los cuales se indican en la Tabla 3-2. 
Tabla 3-2. Clasificación de modelos de planificación energética (Domínguez, 2015).

\begin{tabular}{|l|l|}
\hline \multicolumn{1}{|c|}{ Diferenciación } & \multicolumn{1}{c|}{ Tipos } \\
\hline Objetivo operacional & Simulación, optimización. \\
\hline $\begin{array}{l}\text { Procesamiento y orden de la } \\
\text { información }\end{array}$ & Top-down, bottom-up. \\
\hline Objetivos & $\begin{array}{l}\text { Demanda, oferta, previsión, reducción de emisiones, } \\
\text { expansión de energías renovables. }\end{array}$ \\
\hline Tipo de salida & $\begin{array}{l}\text { Multiple Input Single Output (MISO), en español: } \\
\text { Múltiples Entradas Una Salida. Multiple Input } \\
\text { Multiple Output (MIMO), en español: Múltiples } \\
\text { Entradas Múltiples Salidas. }\end{array}$ \\
\hline
\end{tabular}

Los modelos de simulación son modelos descriptivos, que se basan en la representación lógica de un sistema y que tienen como objetivo reproducir simplificadamente su funcionamiento (Álvarez, 2004). Son principalmente modelos de demanda energética. Los modelos de optimización están orientados a objetivos, pero también contienen elementos descriptivos. El factor más decisivo para este tipo de modelos podría ser el orden de preferencia para un grupo o grupos de nuevas tecnologías. Una comparación de escenarios, suponiendo disponibilidad y no disponibilidad de ciertas tecnologías, puede definir su peso en términos de criterios de objetivos como los costes generales, las importaciones de petróleo o los daños ambientales. Esto es lo que se llama optimización multicriterio (Domínguez, 2015).

En los modelos Top-down, la entrada son los indicadores económicos generales, la información macroeconómica y a partir de esto se estima la demanda primaria, la demanda sectorial y los procesos intermedios. Este tratamiento considera a la tecnología de transformación de la energía como cajas negras, sin representaciones explícitas, incluso suele representarse al sector tecnológico sólo con tablas que contienen unos índices de eficiencia económica. Estos métodos suelen utilizarse para predicciones a corto plazo, o bien para el análisis de sectores específicos, y sólo si no existen discontinuidades en los datos históricos de entrada. En los modelos Bottom-up se analiza el uso final de la energía, la demanda final sectorial, y el modelo estima la demanda primaria a partir de ella. No toma en cuenta las características sintetizadas de la tecnología dentro de un mercado sino los costos directos de uso de dicha tecnología. Estos métodos suelen usarse para determinar sistemas que estén muy bien definidos a largo plazo y si se estima que 
no existen importantes correlaciones entre el sistema energético y otros sistemas económicos (Álvarez, 2004).

Otra diferencia importante entre los modelos energéticos es si están especializados en un tema o aplicación particular, con el objetivo de darnos información sobre la demanda de energía, la oferta de energía, modelos de predicción pura (Diseñados exclusivamente para predecir la energía futura), modelos de reducción de emisiones o modelos de energías renovables (Domínguez, 2015).

Con respecto al tipo de salida de un modelo energético, se diferencian los modelos MISO (Multiple Input Single Output), que utiliza dos o más entradas de energía, tales como nuclear y solar, y produce una sola salida, la mayoría de las veces de electricidad; y los modelos MIMO (Multiple Input Multiple Output), que incluye múltiples entradas y también produce múltiples salidas, como producir energía eléctrica y térmica. Por lo tanto, un modelo MIMO ofrece una alternativa flexible y robusta para el ecosistema energético, debido a su capacidad para operar dinámicamente diversos insumos $\mathrm{y}$ productos con diferentes costes (Binder, et al., 2014).

Además de las características mencionadas, existe otra clasificación de los modelos de planificación energética relacionada a su formulación matemática, pudiendo ser, principalmente, lineales y no lineales. Actualmente, los modelos que usan programación lineal son frecuentemente utilizados para analizar el efecto de distintas políticas energéticas en mercados eléctricos. Una de las grandes ventajas de los modelos que utilizan formulaciones lineales es su simplicidad. Producto de los avances tecnológicos en hardware computacional y software comercial de optimización, hoy es posible resolver grandes problemas de optimización lineales sin mayor dificultad. Como alternativa a los modelos de planificación lineales, existen los modelos no lineales que no consideran variables discretas o enteras. Estos incorporan características más realistas de los sistemas de potencia y mercados eléctricos que los modelos puramente lineales, incluyendo la elasticidad-precio de la demanda eléctrica, pérdidas cuadráticas de transmisión, cambios incrementales a la reactancia de las líneas y costos de operación no lineales. A pesar de los potenciales beneficios de capturar algunas de las no linealidades de los mercados, éstos modelos no se utilizan tan frecuentemente como los modelos lineales mencionados anteriormente. La principal dificultad radica en que el software de optimización no lineal no ha progresado a niveles comparables con el software de optimización lineal, lo que limita los modelos no lineales a aplicaciones relativamente 
pequeñas. Además, el software comercial de optimización no lineal no entrega garantías de que la herramienta será capaz de encontrar una solución óptima local o global, lo que introduce incertidumbre a la selección de planes de inversiones (Universidad Adolfo Ibañez; EECG Consultores, 2017).

Son muchos los modelos de planificación energética que se han desarrollado en los últimos años, por lo tanto, hacer una revisión de todos ellos sería demasiado tedioso. En la presente tesis doctoral se han seleccionados los modelos que resultan de especial interés por su impacto en la investigación operativa y por su relación con el acceso universal a la energía en las CRA.

\subsubsection{Modelos de demanda energética}

\subsubsection{Modelo MEDEE.}

El modelo fue desarrollado por B. Chateau y B. Lapillonne del Instituto Económico y Jurídico de la Energía (IEJE) de la Universidad de Grenoble, Francia. Desde entonces el modelo MEDEE ha evolucionado y se ha adaptado para ajustarse a la modelación de diversos sistemas de demanda de energía. Por ejemplo, B. Lapillonne desarrolló el MEDEE-2 cuando el Instituto Internacional para el Análisis de Sistemas Aplicados (IIASA, Laxenburg, Austria) necesitó realizar estudios de demanda de energía a escala mundial.

Este modelo ha estado en continua evolución, mejorando los instrumentos de análisis, simplificando los sectores cuando era necesario y adaptando su estructura a distintos objetivos finales. Así, también han surgido, el MEDEE-3, el MEDEE región, el MEDEE de utilización racional de la energía, el MEDEE-SUD y el MEDEE-EUR. Todos estos modelos tienen las mismas características. Son modelos a largo plazo (15-20 años) que interrelacionan la demanda de energía final de un país con su desarrollo socioeconómico. La elección de un modelo u otro de la familia MEDEE se realiza en función de dos factores. Cada versión está desarrollada para un ámbito territorial, tipo de economía y nivel de desarrollo, por otro lado, la disponibilidad de datos de consumo, balances, estadísticas, etc., varían la posibilidad de utilizar una versión u otra del modelo. La estructura general de estos modelos es muy parecida, por ello, sólo se va a describir el modelo MEDEE, siendo válido lo expuesto para el resto de la familia. 
El modelo MEDEE es un modelo de simulación, que utiliza la teoría de análisis de sistemas a los problemas energéticos. Permite calcular la demanda energética a nivel desagregado (por sectores y por usos energéticos). Su técnica de modelización se sitúa entre el análisis energético y la econometría. El análisis de la demanda se realiza integrando la macro y la microeconomía, así como los factores económicos y tecnológicos que influyen en la demanda energética a largo plazo. La previsión utiliza la técnica de desarrollo de distintos escenarios de evolución de la sociedad. El sistema se analiza descomponiéndolo en sub módulos más sencillos y homogéneos. Esta división se realiza desde un punto de vista de uso energético, de comportamiento económico y según el contexto tecnológico (García, 2004).

El modelo MEDEE fue adaptado a la región Cataluña (España) para la previsión de la demanda energética a medio y largo plazo (Alario, 1981). En dicha aplicación el análisis del sistema se divide en dos:

- Análisis socio-económico.

- Análisis técnico.

Realizados los escenarios socio-económico y técnico, que constituían el sistema en la región catalana, se dividieron de nuevo en urbano, industrial y agrícola rural. En el caso general del modelo, el sistema socioeconómico se descompone en los siguientes sectores de actividad:

- Industria. Grandes consumidoras de energía e industrias ligeras.

- Agricultura, pesca y construcción.

- Transporte.

- Doméstico y Servicios.

Y el sistema de usos de la energía en:

- Usos productivos. Calor a alta y baja temperatura.

- Materias primas.

- Transporte.

- Electricidad específica.

- Usos domésticos y servicios. Calefacción, agua caliente sanitaria y cocina.

Para determinar la proyección de la demanda energética de cada sistema se definen los correspondientes módulos de cálculo. A su vez, el sistema socio-económico 
dispone de su módulo de cálculo, encargado de proyectar la evolución de las grandes variables socio-económicas.

Esta estructura de previsión de la demanda utiliza para el cálculo la energía útil de cada una de las microunidades del modelo. Su obtención se realiza a través del análisis del sector correspondiente y los factores determinantes de estos servicios (producción en las industrias, pasajeros o carga por kilómetro en transporte, número de viviendas en el sector doméstico, etc.). Si no existe posibilidad de sustitución entre fuentes, el modelo utiliza directamente la energía final. Por último, se realiza el balance en cada sector, siempre reflejado en términos de energía final.

El análisis modular y la simulación de la demanda energética se realizan según un conjunto de hipótesis ordenadas en varios niveles:

a. Entorno internacional (tasa de crecimiento mundial, evolución de los precios del crudo, etc.).

b. Evolución del sistema socioeconómico nacional dentro del entorno definido anteriormente (política industrial y energética, organización del territorio, medio ambiente, etc.).

c. Entorno socioeconómico resultante del nivel anterior (concretado en la estructura de combustibles de la industria, rendimiento de los equipos energéticos, desarrollo de las infraestructuras de transporte, precios de los productos energéticos, etc.).

Estos niveles se sitúan fuera del modelo, siendo el resultado cuantificado de los escenarios lo que se utiliza como entrada en el modelo. De esta forma pueden analizarse los efectos producidos por diferentes políticas sobre la demanda de energía (García, 2004).

\subsubsection{Modelo MAED.}

El MAED (Modelo para el Análisis de la Demanda de Energía) evalúa la demanda futura de energía con base en escenarios de desarrollo socio-económico, tecnológico y demográfico a mediano y largo plazo. El modelo relaciona sistemáticamente la demanda de energía específica para producir varios bienes y servicios identificados en el modelo, con los factores tecnológicos, económicos y sociales correspondientes que afectan esta demanda. La demanda de energía se desagrega en un gran número de categorías de uso final; cada una corresponde a un servicio dado o a la producción de cierto bien. La 
naturaleza y el nivel de la demanda de bienes y servicios son una función de varios factores determinantes, en los que se incluyen el crecimiento de la población, el número de habitantes por vivienda, el número de equipos electrodomésticos usados en la hogares, la movilidad de la población y las preferencias de modos de transporte, las prioridades nacionales para el desarrollo de ciertas industrias o sectores económicos, la evolución de la eficiencia de ciertos tipos de equipamiento, la penetración de nuevas tecnologías o formas de energía en el mercado, etc. Las tendencias futuras que se esperan para estos factores determinantes, que en su conjunto constituyen los "escenarios", se introducen de manera exógena (Organismo Internacional de Energía Atómica, 2007).

La comprensión de estos factores determinantes permite evaluar varias categorías de demanda de energía para cada sector económico considerado. La demanda total de energía para cada categoría de uso final se agrega en cuatro sectores principales “consumidores de energía : Industria (que incluye Agricultura, Construcción, Minería y Manufacturero), Transporte, Servicios y Residencial. El modelo proporciona un sistema de contabilidad sistemático para evaluar el efecto que tendría en la demanda de energía un cambio en la economía o en los estándares de vida de la población.

El punto de partida para usar el modelo MAED es la construcción del patrón de consumo de energía del año base dentro del modelo. Esto requiere la recopilación y conciliación de los datos necesarios de las diferentes fuentes, deducir y calcular varios parámetros de entrada y ajustarlos para reproducir el balance energético en el año base. Este proceso ayuda a ajustar el modelo a la situación específica del país (Organismo Internacional de Energía Atómica, 2007).

\subsubsection{Modelo PROCER}

El modelo PROCER (Proyección de los Consumos Energéticos Representativos) fue desarrollado por el Dr. Joaquín Ortega Costa y sus colaboradores del Ministerio de Industria y Energía para su aplicación en España a finales del siglo XX.

Es un modelo multiplicativo que se basa en la íntima relación existente entre los consumos energéticos y el producto interior bruto (PIB), por lo que se proyectan tanto los consumos energéticos como los valores del PIB al coste de los factores. En el caso regional, se utiliza el valor añadido bruto (VAB) al coste de los factores. El consumo de un sector se considera representativo en la medida que representa la interacción existente 
entre consumo energético y PIB (dividido en los distintos sectores del país). La relación entre el consumo de energía y el Valor Añadido Bruto (VAB) o el PIB no es uniforme: Hay sectores con un gran consumo energético por unidad de valor añadido, mientras que en otros es pequeño (García, 2004).

\subsubsection{Modelo PROCER-CL.}

PROCER-CL (Proyección de los Consumos Energéticos Representativos en Castilla León) fue presentado en la tesis doctoral de D. Francisco Marcos. Se trata de una modificación del modelo PROCER para ser aplicado a una región concreta de España, en la Comunidad de Castilla y León (Benitez, 2015).

\subsubsection{Modelos de oferta energética}

A continuación, se describen algunos de los modelos más representativos de oferta de energía, así como de evaluación de impactos ambientales.

\subsubsection{Modelo BESOM}

El modelo BESOM (Brookhaven Energy System Optimization) ha sido desarrollado por el Brookhaven National Laboratories para la asignación eficiente de recursos en los EE. UU. Y se implementó a nivel nacional para un análisis instantáneo de un futuro puntual en el tiempo. Posteriormente, se desarrollaron varias versiones actualizadas que amplían las capacidades del modelo, incluyendo una vinculación macroeconómica a través de una tabla de insumo-producto (Domínguez, 2015).

BESOM es un modelo de programación lineal, diseñado para la evaluación cuantitativa de tecnologías y políticas energéticas dentro de un marco de sistemas. El modelo está diseñado para examinar las sustituciones entre combustibles en el contexto de limitaciones en la disponibilidad de recursos y tecnologías competidoras, de manera que se pueda elegir la opción más apropiada (Kydes, 1980).

El objetivo de BESOM es minimizar el costo de cumplir con los niveles de demanda medidos en BTU para una serie de usos finales de energía tales como energía térmica o transporte en un año dado. La demanda se satisface mediante la conversión y el transporte de suministros primarios a través de una serie de etapas (Shapiro, et al., 1976). 


\subsubsection{Modelo EFOM}

El modelo EFOM, acrónimo de Energy Flow Optimization Model (Modelo de Optimización del Flujo de Energía), se desarrolló con la capacidad de tomar los datos de salida del modelo MEDEE y realizar la optimización de la oferta energética de una región determinada. Es un modelo de programación lineal, por lo tanto, la optimización se realiza mediante la elaboración de una función objetivo que se optimiza, junto a un conjunto de restricciones, siendo, tanto la función objetivo como las restricciones, ecuaciones lineales. (García, 2004).

El modelo de optimización EFOM ayuda a determinar un mix óptimo de tecnologías para un sistema energético, conforme a un número de condiciones límites tales como límite de emisiones, límite de reducción de costes, etc. El método consiste en realizar primero una verificación del sector de energía primaria que se tendrá en cuenta, podría ser: combustibles fósiles (carbón, petróleo, gas natural, etc.), bio-productos industriales (gases de horno y de cocina) y energías renovables local (biomasa, desechos sólidos y recursos naturales) (Benitez, 2015).

El modelo puede utilizar varias tecnologías, tales como: plantas termoeléctricas convencionales, las plantas de ciclo combinado, paneles fotovoltaicos, sistemas de microturbina, etc., que se han supuesto para la conversión intermedia de la energía primaria. La opción y la adopción de estas tecnologías depende del objetivo del proceso de la optimización; si se les da una mayor importancia a los requisitos económicos, son consideradas las opciones menos costosas. Por otra parte, cuando la meta es reducir emisiones de un agente contaminante, son preferidas las tecnologías más respetuosas al medio ambiente (Benitez, 2015).

Las restricciones a las que se encuentran sometidas las variables del modelo EFOM se clasifican en 6 tipos:

a) Restricciones debidas a los balances de los flujos energéticos. En cualquier nodo de los flujos de un sistema energético, la entrada de energía debe ser igual a la salida de energía más las pérdidas. Dicho de otra forma, la suma de la energía que entra en un proceso ha de ser igual a la suma de la energía saliente más las pérdidas que se produzcan en el proceso (Primer Principio de la Termodinámica). 
b) Restricciones originadas por las ecuaciones de reparto. Para cada tipo de energía o estado de un producto energético (carbón en parque, carbón en caldera, etc.) la proporción de participación de cada uno de los flujos que llegan al mismo es fija y constante para cada período.

c) Restricciones de capacidad. El flujo energético en un proceso en un momento t, no puede superar la capacidad instalada en el año base $T_{0}$ más la prevista desde $\mathrm{T}_{\mathrm{o}}$ hasta $\mathrm{t}$.

d) Restricciones debidas a la política energética (por ejemplo la disminución de la dependencia exterior mediante un aumento de la autoproducción, moratoria nuclear, etc.) o derivadas de las características del sistema energético (limitación de la generación hidroeléctrica debido a la pluviometría, etc.) o debidas a la falta de nuevas tecnologías que deben ser importadas o desarrolladas (tecnologías de uso limpio del carbón, nucleares de última generación, de generación con energías renovables, etc.).

e) Restricciones que limitan la capacidad entre valores máximos y mínimos.

f) Las restricciones exógenas. Se establecen como una combinación lineal de los flujos asociados a los parámetros para los distintos procesos (García, 2004).

\subsubsection{Modelo ESMERALDA}

ESMERALDA (Estudio Macroenergético Regional, Demanda y Abastecimiento) estudia globalmente el suministro energético regional basado en la demanda correspondiente a cada región. De esta manera, los insumos son la oferta y la demanda de las regiones a evaluar (Bustinduy, 1983, citado por Domínguez, 2015, p.86).

Este modelo divide el mundo en 15 regiones energéticas y pretende obtener los datos necesarios para sustituir las energías renovables por convencionales, cuando se consideren posibles y beneficiosas, teniendo en cuenta, entre otros, datos de población, estudios demográficos, cifras per cápita. También describe la producción mundial, el consumo, las exportaciones e importaciones potenciales, alcanzando algunas previsiones a medio y largo plazo (Domínguez, 2015). 


\subsubsection{GEAYL}

El modelo GEAYL (Generación Eléctrica Autóctona y Limpia) fue desarrollado por Benítez (2015) para su aplicación en una comunidad rural aislada de Cuba.

GEAYL considera un modelo para el cálculo de la demanda y otro para la estimación de la oferta. El modelo de demanda, denominado GEAYL-D, proyecta los consumos energéticos hasta el 2030, considerando un incremento del PBI bajo tres escenarios distintos, utilizando para ello los modelos de cálculo sumativo y multiplicativo.

El modelo de oferta, denominado GEAYL-O, incorpora fuentes de energías renovables y no renovables. Es un modelo de optimización de las energías y se evalúan cinco funciones objetivo, las cuales serían las de mayor interés para una comunidad rural no energizada. Estas cinco funciones lineales son compatibles con los criterios utilizados en la mayoría de los modelos de planificación energética. La primera función considera los costes de las tecnologías, las tres siguientes consideran las emisiones de $\mathrm{CO}_{2}, \mathrm{NOx}$ y $\mathrm{SO}_{2}$, y la quinta función considera la aceptación social de las tecnologías propuestas. Los coeficientes de esta última función fueron obtenidos por el método AHP. Dichas funciones son optimizadas individualmente y luego grupalmente haciendo uso de la Programación Compromiso. De este modo se obtuvo la solución Pareto óptima.

\subsubsection{Modelo HOMER}

HOMER (Hybrid Optimization of Multiple Energy Resources) es un modelo de optimización micro energético desarrollado por el Laboratorio Nacional de Energía Renovable de Estados Unidos (NREL) para ayudar en el diseño de micro sistemas de energía y facilitar la comparación de tecnologías de generación de energía en un amplio rango de aplicaciones. HOMER modela tanto el comportamiento físico de un sistema eléctrico como su correspondiente coste en el ciclo de vida, asimismo, permite al modelador comparar muchas opciones de diseño diferentes basadas en indicadores técnicos y económicos (Lambert, Gilman, y Lilienthal, 2006, Rohit y Bhattacharyya, 2014, citado por Domínguez, 2015, p.89).

Realiza tres tareas principales: simulación, optimización y análisis de sensibilidad. En el proceso de simulación, HOMER modela el rendimiento de una configuración de un sistema micro energético particular, cada hora del año para determinar su factibilidad 
técnica y costo de ciclo de vida. En el proceso de optimización, HOMER simula muchas configuraciones de sistemas diferentes, buscando la que satisface las limitaciones técnicas al menor coste del ciclo de vida. En el proceso de análisis de sensibilidad, HOMER realiza optimizaciones múltiples bajo un rango de suposiciones de entrada para evaluar los efectos de incertidumbre o cambios en los insumos del modelo. La optimización determina el valor óptimo de las diversas variables sobre las que el diseñador del sistema tiene control, como la mezcla de componentes que componen el sistema y el tamaño o la cantidad de cada uno. El análisis de sensibilidad ayuda a evaluar los efectos de la incertidumbre o los cambios en las variables sobre las cuales el diseñador no tiene control, como la velocidad media del viento o el futuro precio potencial del combustible (Lambert, Gilman y Lilienthal, 2006, citado por Domínguez, 2015, p.89).

\subsubsection{Modelo IntiGIS}

IntiGIS es una herramienta de escala local desarrollado en 2010 por el grupo de Tecnologías de la Información Geográfica y Energías Renovables (gTIGER) del Centro de Investigaciones Energéticas, Medioambientales y Tecnológicas (CIEMAT), España.

IntiGIS ha sido desarrollada en una aplicación escrita en lenguaje VB.NET y ejecutada sobre ArcGIS 9.3 con la extensión Spatial Analyst. Su principal propósito es servir como herramienta útil en la toma de decisiones, dentro del marco de la planificación integrada de electrificación rural, permitiendo comparar entre una serie de tecnologías de cara a satisfacer la demanda eléctrica existente o estimada en el área de estudio. Biomasa, microhidráulica y minihidráulica quedan al margen. El estudio comparativo se lleva a cabo a través del LEC, un parámetro típico en el análisis de rentabilidad de instalaciones de producción de energía eléctrica. El LEC indica la cuantía de los ingresos necesarios por unidad de energía eléctrica $(€ / \mathrm{kWh})$ producida para recuperar el coste total del sistema durante el tiempo de vida de éste. La ventaja de este índice radica en su capacidad para comparar tecnologías con diferentes tipos de inversión y tiempos de operación (CIEMAT, 2014).

\subsubsection{Modelo IRES}

Kanese-Patil (2007), desarrolló el modelo IRES (Integrated Renewable Energy System) para generar electricidad como producto final, para ser utilizada en sectores 
industriales domésticos, agrícolas, comunitarios y de pequeña escala. Este modelo se basa en un enfoque de programación lineal.

$\mathrm{Su}$ objetivo fue establecer un sistema integrado de energías renovables para la electrificación rural de una red aislada. El modelo consiste en minimizar una función objetivo de costos, sujeta a varias restricciones. La primera igualar la cantidad óptima de los recursos con la cantidad total necesaria a los usuarios finales; y la segunda restricción es la cantidad óptima del valor: "recursos/eficiencia de conversión", que deben ser menor que la disponibilidad de los recursos. Además, en la aplicación realizada por KanesePatil, se han considerado varios escenarios (sistemas IRES) variando el Índice de Energía para encontrar un escenario adecuado para la comunidad aislada en cuestión. De este modo, el modelo reduce al mínimo el coste total de los diferentes sistemas IRES, al tiempo que contabiliza la fiabilidad del sistema (Kanase-Patil, et al., 2010).

\subsubsection{Modelo LEAP}

LEAP (Long-range Energy Alternatives Planning system) es una poderosa y versátil herramienta software para la planificación integrada de la energía y la evaluación de la mitigación del cambio climático desarrollada por el SEI (Stockholm Environment Institute) de los Estados Unidos.

LEAP puede utilizarse en una amplia gama de escalas, desde ciudades y estados hasta aplicaciones nacionales, regionales e incluso globales. Se está convirtiendo rápidamente en un estándar para los países que emprenden la planificación integrada de los recursos y las evaluaciones de mitigación de los GEI, especialmente en los países en desarrollo, y para la creación de estrategias de desarrollo con bajas emisiones (Domínguez, 2015).

\subsubsection{Modelo MARKAL}

MARKAL (MARKet Allocation model) es un modelo desarrollado por la Agencia Internacional de la Energía o IEA (International Energy Agency).

El modelo MARKAL fue desarrollado para hacer análisis de política energética y ambiental con un alcance en tiempo de 40 a 50 años, en periodos de 5 años, para un sistema energético preestablecido. Es un modelo integrado, construido en programación lineal, que hace un análisis Arriba-abajo de la demanda como entrada, y un análisis de la 
oferta, Abajo-arriba, atendiendo a los tipos de tecnología presentes y futuras, como control. Se define un caso de referencia con la demanda estimada y ninguna medida financiera o de abatimiento de emisiones. Después se introducen las medidas de mercado o los porcentajes de reducción requerido y se generan los casos eligiendo las tecnologías por el costo mínimo para la opción. Las salidas típicas son curvas de abatimiento de costo o los costos marginales por caso. Es un modelo que requiere de una gran cantidad de datos, tanto económicos como tecnológicos (Álvarez, 2004).

En términos generales el modelo MARKAL sigue cuatro pasos para su construcción: El primero paso es definir un Sistema Energético de Referencia (Reference Energy System, RES), que represente el sistema energético completo, desde la extracción de recursos, transformación, transmisión, distribución y consumo. La definición de este sistema es la base del modelo, y en el que se representan todas las tecnologías disponibles y todas las relaciones entre oferta y consumo. El segundo paso de la estructura del MARKAL sería definir las condiciones actuales del sistema, su capacidad de expansión y los posibles cambios de tecnología, que podrían darse en el período a analizar. El tercer paso es definir los escenarios posibles, a partir de lo que se conoce como un árbol de eventos, que define para cada periodo de tiempo las posibilidades de evolución del sistema, que suelen ser sólo dos por periodo de tiempo. El cuarto y último paso consistiría en elegir el mejor escenario posible. Esto se hace a partir de técnicas de optimización lineales y no lineales, que buscan los equilibrios parciales entre la oferta y la demanda, y se elige aquel que minimiza el costo de la opción (Álvarez, 2004).

\subsubsection{Modelo MESSAGE}

El modelo de suministro de energía MESSAGE (Model for Energy Supply Systems And their General Environmental impact) fue desarrollado por IIASA (International Institute for Applied Systems Analysis).

MESSAGE es un modelo de programación lineal dinámica que minimiza los costes totales del suministro de energía en un horizonte temporal determinado.

Consiste en equilibrar la demanda de energía secundaria (o final), que se desagrega en sectores y es exógena al modelo, con el suministro de energía primaria, dado como un conjunto de disponibilidades de recursos que se desglosan en un número opcional de categorías de costos al elegir entre un conjunto dado de tecnologías de 
conversión de energía. Las limitaciones de los modelos reflejan la limitada velocidad de acumulación de tecnologías, la limitada disponibilidad anual de recursos y las relaciones tecnológicas, entre otros aspectos del sistema energético. El modelo permite la definición de regiones de carga (por ejemplo, para la demanda de electricidad), distingue entre recursos autóctonos e importados, toma en cuenta el impacto ambiental de las estrategias de suministro de energía y opcionalmente incluye este impacto en la función objetivo (Schrattenholzer, 1981).

\subsubsection{Modelo NEMS}

El NEMS (National Energy Modeling System) es un modelo desarrollado por la EIA (Energy Information Administration). Se trata de un modelo modular flexible que representa el sistema energético de EE.UU. Representa a un modelo de tercera generación de previsión a mediano plazo que se desarrolló desde 1974, en el predecesor de la EIA, la Administración Federal de Energía, y que analizaban el sector energético-económico interno para el país norteamericano. Antes del NEMS, se utilizó de 1982 al 1993 el modelo IFFS (Sistema de Previsión para el Futuro Intermedio, Intermediate Future Forecasting System), y antes de este periodo se utilizó el MEFS (Sistema de Previsión Energética de Mediano plazo, Midterm Energy Forecasting System). Es un modelo nacional, por tanto, que divide al país norteamericano en 9 sub-regiones, no en los 50 estados independientes, pero que al mismo tiempo trata de representar el mercado internacional de la energía, para reforzar su validez. Consideran que su alcance en el tiempo es de mediano plazo, generalmente 20 años. Para el procesamiento matemático utiliza la Programación lineal (Álvarez, 2004).

\subsubsection{Modelo OREM}

El modelo OREM (Optimal Renewable Energy Model) fue desarrollado por Iniyan et al., para una utilización eficaz de las fuentes de energía renovables en la India para el año 2020-21.

OREM es un modelo de programación lineal. Optimiza los suministros de energía a través de fuentes renovables. Los factores primarios, tales como costo y la eficiencia, se consideran en la función objetivo. Por lo tanto, el costo de sistemas de las energías renovables debe ser minimizado y la eficiencia de los sistemas de energías renovables debe ser maximizada. Las opciones energéticas renovables que son tenidas en cuenta son 
todas aquellas aplicables a los diferentes sectores en la región. Las restricciones que usa del modelo son la aceptación social, la fiabilidad, el potencial y la demanda. Con el fin de determinar el factor de aceptación social de los sistemas de energía renovable para diferentes usos finales, se realizó un estudio Delphi. El análisis de fiabilidad se hizo para averiguar el factor de fiabilidad de la planta de energía solar fotovoltaica y el generador de turbina eólica que fueron considerados en el estudio (Iniyan, et al., 1998).

\subsubsection{Modelo PAMER}

El modelo PAMER (Planificación con Modelo de Energías Renovables) fue desarrollado por Marcos (1984). Es un modelo de planificación energética de oferta que permite la sustitución parcial de las fuentes energéticas convencionales demandadas en los distintos sectores consumidores de una región, por energías renovables.

PAMER es un modelo que mezcla la Programación Lineal con las técnicas blandas ya que utiliza estas para determinar los coeficientes que aparecen en la función objetivo, la cual es lineal al igual que las restricciones. La función objetivo empleada maximiza la cantidad de energías proveniente de fuentes renovables. La novedad con respecto a otros modelos de planificación energética es que los coeficientes o parámetros de la función lineal se determinan por la opinión de expertos. Y, a partir de la opinión de estos expertos, se aplican las llamadas técnicas blandas, como la técnica de las precedencias o de la distancia al punto ideal, para establecer las prioridades entre las distintas fuentes.

Las restricciones son de tres tipos: El primero, el límite de energías renovables, suponiendo que éstas solo pueden sustituir un determinado porcentaje de la demanda que cubre las fuentes convencionales en un sector dado; el segundo, tecnológico, que provienen de las dificultades de captación, distribución, almacenaje y aprovechamiento, de cada tipo de energía renovable; y el tercero, energías convencionales que ya han sido sustituidas y tecnologías de energías renovables ya instaladas, estas limitaciones suponen que existe una cantidad mínima de energía ya instalada. Por tanto, la contribución de cada tipo de energía renovable no puede ser inferior que la cantidad instalada.

La innovación de este modelo, como se ha señalado, se encuentra en la utilización de técnicas de análisis vectorial ("técnicas blandas") para el establecimiento de los coeficientes de la función objetivo que se va a optimizar ("técnica dura"). El análisis 
vectorial se realiza a partir de parámetros sociales, técnicos, económicos y medioambientales que permiten jerarquizar las diferentes fuentes de energía renovables (Benitez, 2015).

\subsubsection{Modelo POLES}

POLES (Prospective Outlook on Long term Energy Strategy) fue desarrollado en 1997 dentro del programa Joule III de la Comisión Europea. Es un modelo que tiene un alcance de 30 años, está diseñado para determinar la demanda de energía, crear escenarios de precios y proyecciones de suministro. Entre sus objetivos se encuentran:

- Reducir incertidumbre e identificar los márgenes de libertad en el futuro desarrollo del consumo energético y emisiones correspondientes de $\mathrm{CO}_{2}$.

- Proveer de elementos para análisis de eficiencia de costos en las políticas de reducción de emisiones.

- Analizar el impacto de la entrada de tecnología o sistemas de reducción de emisiones en los precios.

- Ayudar a identificar las mejores estrategias de inversión y desarrollo para la Unión Europea en el contexto mundial.

El POLES utiliza más de 60 mil parámetros: 35 mil definiciones, 15 mil datos exógenos y 10 mil variables exógenas. Representa 14 países individualmente y 12 regiones locales, todo se agrega en hace 26 regiones globales. Los sectores analizados se dividen en 11 actividades. Los datos de entrada proceden tanto de la IEA, del EuroStat y del Banco Mundial (Álvarez, 2004).

POLES se divide en dos grandes segmentos generales de cómputo: por un lado, hay una integración vertical que calcula la oferta y demanda energética de cada región, y después se integran los resultados de cada región en la representación del mercado internacional de energéticos primarios, carbón, petróleo y gas únicamente. A esta última integración se le considera horizontal. En términos generales las dos entradas principales por región son la población y el PIB, con lo que se determina la demanda final por sectores de consumo, con esto, primero se considera que los energéticos naturales suplen algo de la demanda final y lo sobrante pasa al módulo de electricidad y sector transformación, con lo que se determina la necesidad de importaciones y exportaciones de energéticos 
primarios para cada región. Las curvas de importación y exportación de cada región se combinan en la simulación del mercado internacional de energía (Álvarez, 2004).

\subsubsection{Modelo SAGE}

SAGE (System for the Analysis of Global Energy markets) es un modelo integrado de varios modelos regionales desarrollado por la EIA (Energy Information Administration). El modelo SAGE necesita, al igual que el MARKAL, definir un Sistema Energético de Referencia, RES, por cada una de las 15 regiones mundiales que contempla, y que comprende hasta 42 usos finales de energía, que integran la demanda del sistema. Sin embargo, se hace explicito que el esquema de definición de evolución de dicho sistema se hace siguiendo los siguientes factores:

- Inversión en tecnologías

- Niveles de operación y mantenimiento por tecnología

- Accesibilidad de recursos primarios de energía

- Importación/exportación entre regiones

Cabe mencionar que las 15 regiones que elige el SAGE no se definen a partir de ningún criterio previo aparente. Siguiendo con la política ya establecida del EIA, se buscaba representar principalmente a 14 países individualmente, 9 considerados desarrollados y 5 subdesarrollados, y enmarcarlos dentro de regiones geográficas más o menos coherentes que después se sintetizarían en: Norte América, Europa occidental, Asia industrializada, Europa oriental y ex URSS, Centro y Sur América, Asia en desarrollo, Oriente Medio y por último África (Álvarez, 2004).

Es un modelo con un largo alcance de tiempo, que puede contemplar hasta once periodos de cinco años, sin embargo, dentro del periodo específico se tienen 6 tramos temporales dependiendo del nivel de representación que exija cada uso-final o tecnología. Los cinco años del periodo de iteración se tratan con las mismas consideraciones, salvo en el caso de las inversiones de tecnología que se asumen siempre ocurriendo en el primer año del periodo. En general las proyecciones del consumo de energía se hacen para cubrir una demanda prevista basándose en los patrones de uso de energía de cada región, las previsiones de crecimiento económico por región, el tipo de equipo que consume la energía y sus características y las fuentes de energía disponibles y las previstas. El sistema busca un equilibrio parcial entre oferta y demanda, buscando el costo mínimo por región. 
Se consideran inversiones, costos de operación, mantenimiento, costos de producción e importación, ganancias por exportaciones, costo de traslado, pérdidas y finalmente impuestos y subsidios. Esto para cada región y cada periodo por lustro. Todo en un ambiente de programación lineal.

Las restricciones del sistema son: Satisfacción de la demanda, conservación de inversión, uso de la capacidad instalada, balance entradas-salidas por región, balance de la electricidad y la calefacción, picos eléctricos, capacidad eléctrica base, factor de disponibilidad estacional, participación en cesta y restricción de emisiones. De este último punto se hace una estimación de las emisiones totales por región (Álvarez, 2004).

\subsubsection{Modelo SEMA}

El modelo SEMA (Sustitución Energética Multiatributo) es un modelo de oferta construido a partir del modelo PAMER. SEMA es un modelo de programación lineal que se basa en la Programación Multiobjetivo y Multi-atributo como métodos de solución, fue desarrollado por García (2004) para su aplicación a la Comunidad Autónoma de Madrid. Este modelo fue uno de los que sirvió como base para el desarrollo del modelo propuesto en la presente tesis doctoral.

Considera tres funciones objetivo: función de maximización de las energías renovables; función de minimización de las emisiones de $\mathrm{CO}_{2}$ y función de minimización de costes. Los coeficientes de la función objetivo que maximiza las energías renovables, fueron calculados a través de técnicas vectoriales que tienen en cuenta criterios económicos, sociales, etc. dados por expertos; los de la función de minimización de emisiones son coeficiente relacionados a las emisiones evitadas de $\mathrm{CO}_{2}$ y la función minimización de costes utiliza coeficientes de coste por unidad de energía producida.

Del mismo modo, las restricciones consideradas fueron cuatro: de demanda energética eléctrica, de demanda energética no eléctrica, de potencial total de energías renovables y, por último, de cantidad de energías renovables existentes. Finalmente se optimizaron las funciones objetivo por separado y fue aplicada la técnica multicriterio Programación Compromiso para la búsqueda de la solución óptima (Benitez, 2015).

\subsubsection{Modelo SRIME}


El modelo SRIME (Sustainable \& Renewable Implementation Multi-criteria Energy model) fue desarrollado por Domínguez (2015) para la planificación energética de Sri Lanka.

SRIME contempla el estudio de la demanda de energía y el potencial aprovechable de las energías renovables del país. Asimismo, se evalúan las emisiones de $\mathrm{CO}_{2}, \mathrm{NOx}$ y $\mathrm{SO}_{2}$, teniendo en cuenta las emisiones de ciclo de vida de las tecnologías energéticas renovables consideradas. Además, considera el estudio de las interacciones de las energías renovables en salud y educación, con el fin de beneficiar estos sectores de desarrollo en las zonas rurales. De este modo, se formulan seis funciones objetivo que se optimizan independientemente, bajo un enfoque multi-criterio: maximización de la cantidad reemplazable de combustible fósil por energías renovables; maximización de las emisiones evitadas de $\mathrm{CO}_{2}$, $\mathrm{NOx}$ y $\mathrm{SO}_{2}$; maximización del desarrollo de la educación y la salud rural; y la minimización de los costes.

Las funciones objetivo fueron optimizadas globalmente a través de la minimización de la Programación Compromiso, haciendo uso de la distancia Chebyshev, obteniendo la solución óptima de Pareto. Finalmente, fueron dados diferentes pesos a las seis funciones para tener un ejemplo de las muchas combinaciones disponibles, con el fin de que los encargados de la toma de decisiones decidan qué función objetivo recibirá una especial importancia en comparación con las demás y puedan obtener la priorización de las alternativas energéticas consideradas. Este análisis fue también aplicado en el modelo que se propone en la presente tesis doctoral.

\subsubsection{Modelo SURE}

SURE (Sustainable Rural Energy) es un software diseñado por el proyecto de investigación sobre energía renovable para medios de vida rurales sostenibles (RESURL), financiado por el Departamento de Desarrollo Internacional del Reino Unido (DFID UK), que intenta abordar el problema de la provisión de energía rural considerando la inclusión de factores económicos y/o ambientales. RESURL reconoce que, para aumentar las posibilidades de éxito de introducir plenamente y de manera sostenible la provisión de energía adecuada en las zonas rurales remotas, antes de cualquier instalación del sistema energético, debe realizarse una evaluación exhaustiva de las condiciones y requisitos locales y regionales. De esta manera, SURE intenta facilitar las decisiones que promueven la infraestructura energética planificada con el objetivo de mejorar a largo plazo los 
medios de vida rurales, prestando también atención a los aspectos no técnicos de la provisión de energía. RESURL proporciona un enfoque sistemático y multidimensional, teniendo en cuenta los impactos sociales, financieros, ambientales y humanos de un proyecto energético determinado (Cherni, et al., 2007).

Los aspectos particularmente útiles de SURE es que permite representaciones gráficas de cambios y compensaciones como pueden ocurrir durante la implementación de diferentes alternativas energéticas; también incluye aspectos técnicos y no técnicos de la energía y los medios de subsistencia; Y finalmente, SURE se refiere implícitamente a las dimensiones de tiempo de 'antes' y 'después' de una intervención energética que se pone en marcha. El software SURE utiliza el método multi-criterio de Programación Compromiso con $\mathrm{p}=2\left(\mathrm{~L}_{2}\right)$ para la toma de decisiones y los pesos (Cherni, et al., 2007). Cherni et al. aplicaron el modelo SURE en una comunidad rural aislada de Colombia. El objetivo del trabajo fue la selección de alternativas energéticas renovables satisfactorias para una comunidad rural parcial o no electrificada. Para ello SURE establece el valor de cinco Indicadores de Capitales (IC) de la comunidad (por una encuesta participativa y por observaciones). El objetivo es que cada IC alcance un valor cercano a 1, que es el estado ideal para que la comunidad sea sostenible. Esto supone, según la teoría de Medios de Vidas Sostenibles, que solo se alcance a través de la energización. Se evalúa cada alternativa energética propuesta contra cada IC. Se ordenan estas alternativas que mejor influyan en los IC a través de la Programación Compromiso (Benitez, 2015).

\subsubsection{Modelo TESOM}

El modelo TESOM (Timed Stepped Energy System Optimization Model) fue desarrollado por el Brookhaven National Laboratory, junto con el BESOM previamente establecido. TESOM asigna el suministro de energía de acuerdo a las demandas de energía, de manera que el costo puede minimizarse, presentando una representación única de la generación de electricidad. Esto se realiza a través de la programación lineal. Una vez que el modelo reconoce las fuentes de energía disponibles, las capacidades de conversión y la eficiencia, y los costes operativos tecnológicos correspondientes, la optimización del sector energético calcula los diferentes suministros de energía que minimizan el coste (Jorgenson, 1998, Kydes \& Rabinowitz, 1981, citado por Domínguez, 2015, p.124). 
Este modelo se ha utilizado para evaluar y optimizar las sustituciones entre combustibles en el contexto de limitaciones de la disponibilidad de recursos y tecnologías competidores (Jebaraj \& Iniyan, 2006, citado por Domínguez, 2015, p.124).

\subsubsection{Modelo TIMES}

El modelo TIMES (The Integrated MARKAL-EFOM System) se ha desarrollado a partir de los modelos MARKAL y EFOM.

TIMES es un modelo de programación lineal que representa todo el sistema energético de un país o una región. Tal sistema incluye la extracción, transformación, distribución, uso final y comercio de diversas formas de energía y algunos materiales.

Cada sector económico se describe mediante tecnologías, cada una de las cuales se caracteriza por sus parámetros económicos y tecnológicos. Las demandas de uso final (es decir, servicios energéticos) en el caso base se basan en supuestos socioeconómicos y son especificadas exógenamente por el usuario en unidades físicas (número de casas, área comercial, producción industrial, vehículos-kilómetros, etc.) sobre un horizonte futuro. Sin embargo, contrariamente a los modelos tradicionales bottom-up, TIMES reconoce que las demandas son elásticas a sus propios precios. Esta característica asegura la variación endógena de las demandas en aplicaciones con restricciones (sobre emisiones o concentraciones), capturando así la gran mayoría de las retroalimentaciones macroeconómicas en el sistema energético. TIMES calcula un equilibrio parcial intertemporal dinámico en los mercados energéticos integrados. La función objetivo para maximizar es el superávit total. Esto equivale a minimizar el coste total del sistema respetando las limitaciones ambientales y técnicas. Este costo incluye los costos de inversión, los costos de operación y mantenimiento, más los costos de los combustibles importados, menos los ingresos de los combustibles exportados, menos el valor residual de las tecnologías al final del horizonte, más la pérdida de bienestar debido a las reducciones endógenas de la demanda (Vaillancourt, et al., 2008).

Además, TIMES incluye un módulo climático que calcula el impacto de las decisiones energéticas sobre las emisiones y concentraciones de GEI, así como sobre los cambios resultantes en el forzamiento atmosférico y en la temperatura global (Loulou \& Labriet, 2008). Los resultados del modelo principal son inversiones futuras y actividades de tecnologías en cada período. Una salida adicional del modelo es el precio implícito de 
cada forma de energía, material y emisión, que es igual a su costo de oportunidad (precio sombra) (Vaillancourt, et al., 2008).

\subsubsection{Modelo VIPOR}

VIPOR es un modelo de optimización para el diseño de sistemas de electrificación de comunidades rurales. Dado un mapa de un pueblo y alguna información sobre el tamaño de las cargas y los costes del equipo, VIPOR decide qué casas deben ser alimentadas por sistemas energéticos aislados (como los sistemas solares) y cuales deben incluirse en una red de distribución centralizada. La red de distribución está diseñada de forma óptima, teniendo en cuenta el terreno local. A cada punto de carga se le asigna un tipo de carga y el modelador puede definir tantos tipos de carga como sea necesario. Para cada tipo de carga, se debe especificar la demanda eléctrica diaria promedio, tanto en la red como fuera de la red.

La definición de los recursos disponibles es probablemente la parte más difícil de usar VIPOR. El modelo VIPOR se preocupa por el coste total de la electrificación, incluyendo la generación y la distribución. Los costes de generación son una parte importante del análisis. VIPOR busca la configuración óptima, considera sistemas aislados de diferentes dimensiones y usa esta información para calcular los costes resultantes (Domínguez, 2015).

\subsubsection{Modelo WEM}

WEM (World Energy Model) es un modelo matemático utilizado por la IEA (International Energy Agency) que puede aplicarse tanto a nivel global como regional. WEM puede generar pronósticos sectoriales y regionales a medio y largo plazo en materia de demanda energética y generación de energía. Este modelo también puede estimar las inversiones, calcular los factores de contenido de $\mathrm{CO}_{2}$ para el carbón, el petróleo y el gas para diferentes sectores y regiones, así como hacer proyecciones de demanda para tres escenarios posibles (Karjalainen et al, 2014, citado por Domínguez, 2015, p.135).

También puede evaluar los efectos de medidas políticas y los cambios tecnológicos, es decir, bajo escenarios alternativos es posible analizar el impacto de las acciones de política y los avances tecnológicos en la demanda de energía, la oferta, el comercio, las inversiones y las emisiones potenciales (IEA, 2012, citado por Domínguez, 2015, p.135). 
En las Tablas 3-3 y 3-4 se indica el resumen de los modelos de demanda y de oferta, respectivamente, revisados en la presente tesis doctoral. Asimismo, se indican el autor del modelo, el país de origen, el año en el que fue desarrollado y sus características principales. 
Tabla 3-3. Modelos de planificación de demanda energética revisados en la presente tesis doctoral.

\begin{tabular}{|c|c|c|c|c|c|c|}
\hline Modelo & Autor & Institución & País & Año & Técnica utilizada & Características \\
\hline MEDEE & Chateau y Lapillonne & Universidad de Grenoble & Francia & 1978 & Enfoque de escenario & $\begin{array}{c}\text { Simulación, Bottom-up, } \\
\text { MIMO }\end{array}$ \\
\hline MAED & Chateau y Lapillonne & Universidad de Grenoble & Francia & 1978 & Enfoque de escenario & $\begin{array}{c}\text { Simulación, Bottom-up, } \\
\text { MIMO }\end{array}$ \\
\hline PROCER & Joaquín Ortega Costa & $\begin{array}{c}\text { Universidad Politécnica de } \\
\text { Madrid }\end{array}$ & España & 1980 & $\begin{array}{l}\text { Proyección del } \\
\text { PIB/VAB }\end{array}$ & $\begin{array}{c}\text { Simulación, Top-down, } \\
\text { MISO }\end{array}$ \\
\hline PROCER-CL & Francisco Marcos & $\begin{array}{c}\text { Universidad Politécnica de } \\
\text { Madrid }\end{array}$ & España & 1984 & $\begin{array}{l}\text { Proyección del } \\
\text { PIB/VAB }\end{array}$ & $\begin{array}{c}\text { Simulación, Top-down, } \\
\text { MISO }\end{array}$ \\
\hline
\end{tabular}

Tabla 3-4. Modelos de planificación de oferta energética revisados en la presente tesis doctoral.

\begin{tabular}{|c|c|c|c|c|c|c|}
\hline Modelo & Autor & Institución & País & Año & Técnica utilizada & Características \\
\hline BESOM & $\begin{array}{c}\text { Brookhaven National } \\
\text { Laboratories (BNL) }\end{array}$ & $\mathrm{BNL}$ & EE.UU. & 1980 & Programación lineal & $\begin{array}{c}\text { Optimización, Bottom-up, } \\
\text { MIMO }\end{array}$ \\
\hline EFOM & $\begin{array}{l}\text { Comisión de la } \\
\text { Comunidad Europea }\end{array}$ & $\begin{array}{c}\text { Comisión de la } \\
\text { Comunidad Europea }\end{array}$ & Internacional & 1981 & Programación lineal & $\begin{array}{c}\text { Optimización, Bottom-up } \\
\text { MIMO }\end{array}$ \\
\hline ESMERALDA & Joaquín Ortega & $\begin{array}{c}\text { Universidad } \\
\text { Politécnica de Madrid }\end{array}$ & España & 1983 & Enfoque de escenario & $\begin{array}{c}\text { Simulación, Bottom-up } \\
\text { MIMO }\end{array}$ \\
\hline GEAYL & Lázaro Benítez & $\begin{array}{c}\text { Universidad } \\
\text { Politécnica de Madrid }\end{array}$ & España & 2015 & $\begin{array}{l}\text { Optimización } \\
\text { multicriterio }\end{array}$ & $\begin{array}{c}\text { Optimización, Bottom-up } \\
\text { MIMO }\end{array}$ \\
\hline
\end{tabular}




\begin{tabular}{|c|c|c|c|c|c|c|}
\hline Modelo & Autor & Institución & País & Año & Técnica utilizada & Características \\
\hline HOMER & $\begin{array}{l}\text { National Renewable } \\
\text { Energy Laboratory }\end{array}$ & NREL & EE.UU. & 2000 & $\begin{array}{l}\text { Algoritmos de } \\
\text { optimización }\end{array}$ & $\begin{array}{c}\text { Simulación/Optimización, } \\
\text { Bottom-up MISO }\end{array}$ \\
\hline IntiGis & $\begin{array}{c}\text { Centro de } \\
\text { Investigaciones } \\
\text { Energéticas, } \\
\text { Medioambientales y } \\
\text { Tecnológicas } \\
\text { (CIEMAT) }\end{array}$ & CIEMAT & España & 2010 & $\begin{array}{l}\text { Algoritmos de } \\
\text { optimización }\end{array}$ & $\begin{array}{c}\text { Optimización, Bottom-up } \\
\text { MISO }\end{array}$ \\
\hline IRES & Amar Kanase-Patil & $\begin{array}{l}\text { Instituto Indio de } \\
\text { Tecnología }\end{array}$ & India & 2007 & Programación lineal & $\begin{array}{c}\text { Optimización, Bottom-up } \\
\text { MISO }\end{array}$ \\
\hline LEAP & $\begin{array}{l}\text { Stockholm } \\
\text { Environment Institute } \\
(\mathrm{SEI})\end{array}$ & SEI & EE.UU. & 1995 & $\begin{array}{l}\text { Algoritmos de } \\
\text { optimización }\end{array}$ & $\begin{array}{c}\text { Optimización, Bottom-up } \\
\text { MISO }\end{array}$ \\
\hline MARKAL & $\begin{array}{l}\text { Brookhaven National } \\
\text { Laboratories (BNL) }\end{array}$ & $\begin{array}{c}\text { International Energy } \\
\text { Agency (IEA) }\end{array}$ & EE.UU. & 1980 & Programación lineal & $\begin{array}{c}\text { Optimización, Top-down } \\
\text { Bottom-up, MIMO }\end{array}$ \\
\hline MESSAGE & $\begin{array}{l}\text { International Institute } \\
\text { for Applied Systems } \\
\text { Analysis (IIASA) }\end{array}$ & IIASA & Austria & 1981 & Programación lineal & $\begin{array}{c}\text { Optimización, Top-down } \\
\text { Bottom-up, MIMO }\end{array}$ \\
\hline NEMS & $\begin{array}{l}\text { Energy Information } \\
\text { Administration (EIA) }\end{array}$ & $\begin{array}{c}\text { Departamento de } \\
\text { Energía de los Estados } \\
\text { Unidos }\end{array}$ & EE.UU. & 1993 & Programación lineal & $\begin{array}{c}\text { Optimización, Top-down, } \\
\text { MIMO }\end{array}$ \\
\hline OREM & $\begin{array}{l}\text { Iniyan S., Suganthi L. } \\
\text { y Jagadeesan T.R. }\end{array}$ & Universidad de Anna & India & 1998 & Programación lineal & $\begin{array}{c}\text { Optimización, Bottom-up, } \\
\text { MIMO }\end{array}$ \\
\hline PAMER & Francisco Marcos & $\begin{array}{c}\text { Universidad } \\
\text { Politécnica de Madrid }\end{array}$ & España & 1984 & Programación lineal & $\begin{array}{c}\text { Optimización, Bottom-up, } \\
\text { MISO }\end{array}$ \\
\hline
\end{tabular}




\begin{tabular}{|c|c|c|c|c|c|c|}
\hline Modelo & Autor & Institución & País & Año & Técnica utilizada & Características \\
\hline POLES & Programa Joule III & $\begin{array}{c}\text { Comisión de la } \\
\text { Comunidad Europea }\end{array}$ & Internacional & 1997 & Programación lineal & $\begin{array}{c}\text { Simulación, Bottom-up, } \\
\text { MISO }\end{array}$ \\
\hline SAGE & $\begin{array}{c}\text { Energy Information } \\
\text { Administration (EIA) }\end{array}$ & $\begin{array}{c}\text { Departamento de } \\
\text { Energía de los Estados } \\
\text { Unidos }\end{array}$ & EE.UU. & 1998 & Programación lineal & $\begin{array}{c}\text { Simulación, Bottom-up, } \\
\text { MISO }\end{array}$ \\
\hline SEMA & Luis García Benedicto & $\begin{array}{c}\text { Universidad } \\
\text { Politécnica de Madrid }\end{array}$ & España & 2003 & $\begin{array}{l}\text { Optimización } \\
\text { multicriterio }\end{array}$ & $\begin{array}{c}\text { Optimización, Bottom-up, } \\
\text { MIMO }\end{array}$ \\
\hline SRIME & $\begin{array}{c}\text { Luis Carlos } \\
\text { Domínguez-Dafauce }\end{array}$ & $\begin{array}{l}\text { Universidad Europea } \\
\text { de Madrid }\end{array}$ & España & 2015 & $\begin{array}{l}\text { Optimización } \\
\text { multicriterio }\end{array}$ & $\begin{array}{c}\text { Optimización, Bottom-up, } \\
\text { MIMO }\end{array}$ \\
\hline SURE & $\begin{array}{l}\text { Renewable Energy for } \\
\text { Sustainable Rural } \\
\text { Livelihoods } \\
\text { (RESURL) }\end{array}$ & RESURL & Reino Unido & 2006 & Análisis multicriterio & $\begin{array}{c}\text { Simulation, Bottom-up, } \\
\text { MIMO }\end{array}$ \\
\hline TESOM & $\begin{array}{l}\text { Brookhaven National } \\
\text { Laboratories (BNL) }\end{array}$ & BNL & EE.UU. & 1980 & Programación lineal & $\begin{array}{c}\text { Optimización, Bottom-up, } \\
\text { MIMO }\end{array}$ \\
\hline TIMES & $\begin{array}{l}\text { Energy Technology } \\
\text { Systems Analysis } \\
\text { Program (ETSAP) }\end{array}$ & IEA & Internacional & 1984 & Programación lineal & $\begin{array}{c}\text { Optimización, Bottom-up, } \\
\text { MIMO }\end{array}$ \\
\hline VIPOR & $\begin{array}{l}\text { National Renewable } \\
\text { Energy Laboratory } \\
\text { (NREL) }\end{array}$ & NREL & EE.UU. & 2001 & $\begin{array}{l}\text { Algoritmos de } \\
\text { optimización }\end{array}$ & $\begin{array}{c}\text { Optimización, Bottom-up, } \\
\text { MISO }\end{array}$ \\
\hline WEM & $\begin{array}{c}\text { International Energy } \\
\text { Agency (IEA) }\end{array}$ & IEA & Internacional & 1993 & Enfoque de escenario & $\begin{array}{c}\text { Simulación, Top-down, } \\
\text { MISO }\end{array}$ \\
\hline
\end{tabular}




\section{CAPÍTULO IV}

\section{PROPUESTA DE UN MODELO DE PLANIFICACIÓN ENERGÉTICA}

\subsection{Modelo propuesto: Modelo de planificación energética SEPLAN}

Tal como se ha indicado en el Capítulo II, el objeto de la presente tesis doctoral es la elaboración un modelo de planificación energética que tenga en cuenta a las CRA empleando técnicas multicriterio multiobjetivo. El modelo de planificación propuesto ha sido SEPLAN (Sustainable Energy PLANning model).

SEPLAN ha sido desarrollado en base al modelo SRIME (Domínguez, 2015), el cual utiliza la metodología de los modelos SEMA (García, 2004) y PAMER (Marcos, 1985).

SEPLAN ha sido construido mediante el uso de técnicas matemáticas de la teoría de decisión multicriterio, combinadas con las técnicas de análisis vectorial. Consta de 6 funciones objetivo que atienden a: i) maximizar la sustitución de combustibles fósiles por energías renovables, ii) maximizar las emisiones evitadas de $\mathrm{CO}_{2}, \mathrm{NO}_{\mathrm{x}}$ y $\mathrm{SO}_{2}$ y iii) minimizar los costes. Estas funciones objetivo se han tomado de los modelos SRIME y SEMA. Por su parte, SEPLAN incorpora una sexta función objetivo adicional, que evalúa la mejor alternativa energética renovable para el suministro sostenible de energía en las CRA.

Para el desarrollo del modelo, en la primera etapa se considera la estimación de la oferta y la demanda energética en la zona de estudio.

En la segunda etapa se construyen las funciones objetivo, para ello se han definido previamente las alternativas energéticas a considerar en la planificación. Esta etapa considera también el establecimiento de las restricciones del modelo.

La tercera etapa del proceso consiste en la optimización independiente de cada función objetivo mediante programación lineal, obteniéndose los valores ideales y antiideales. Finalmente, el conjunto de soluciones óptimas se obtiene minimizando las distancias al punto ideal $\left(\mathrm{L}_{1}, \mathrm{~L}_{\infty} \mathrm{y} \mathrm{L}_{\mathrm{k}}\right)$.

Es importante mencionar que, al igual que en otros modelos de planificación, las reuniones de los decisores y de los expertos, son fundamentales para priorizar las 
tecnologías más adecuadas según la realidad local, con el objetivo de hacer un uso adecuado de los recursos. Otro aspecto importante es la retroalimentación del modelo una vez obtenidos los resultados preliminares del proceso de optimización, lo cual asegura que los valores obtenidos en la planificación estén de acuerdo a la realidad de la zona de estudio.

Adicionalmente, después de la obtención de los resultados, con el fin de tener un alcance de la robustez del modelo, se ha considerado la realización de un análisis de sensibilidad.

En el diagrama de la Figura 4-1, se indica la secuencia de los procesos para el desarrollo del modelo.

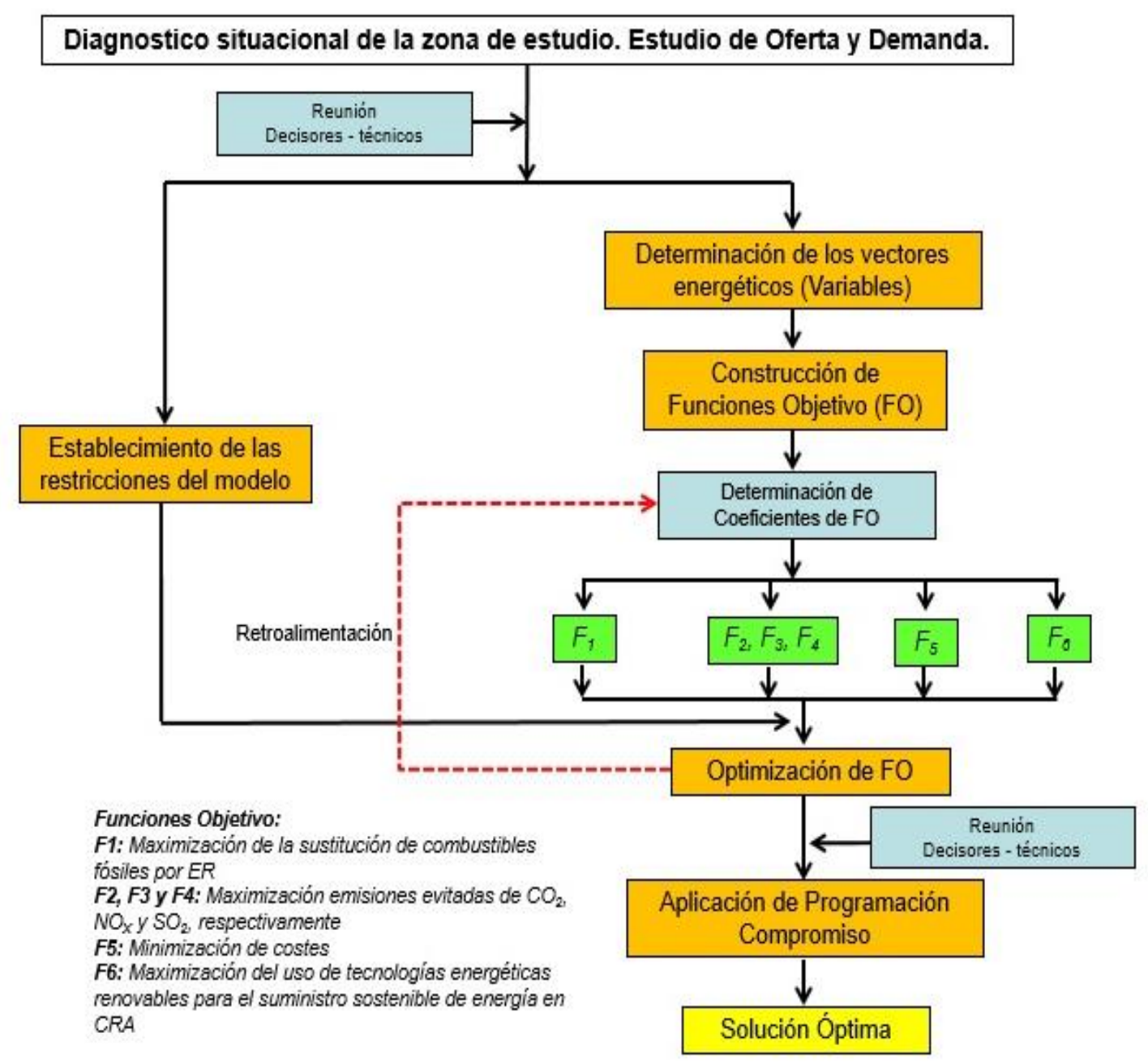

Figura 4-1. Diagrama del modelo SEPLAN. 
4.2 Diagnostico situacional de la zona de estudio. Estudio de Oferta y Demanda.

\subsubsection{Estimación de la Demanda}

Para la estimación de la demanda se tuvo en cuenta la metodología empleada en el modelo PROCER, que considera los modelos sumativo y multiplicativo para el cálculo de la demanda de energía de una región.

Los modelos sumativo y multiplicativo tienen el siguiente método de cálculo:

$$
\begin{gathered}
\text { ModSum }=\text { ConABas }(1+(\mathrm{AA}-\mathrm{AB}) \cdot \Delta \mathrm{PIB}) \\
\text { ModMult }=\text { ConABas }^{(1+(\mathrm{AA}-\mathrm{AB}) \cdot \Delta \mathrm{PIB})}
\end{gathered}
$$

Donde:

ModSum: Modelo Sumativo

ModMult: Modelo Multiplicativo

ConsABas: Consumo Año Base

AA: Año Analizado

AB: Año Base

PIB: Producto Bruto Interno

$\Delta$ PIB: Aumento del Producto Interno Bruto

\subsubsection{Estimación de la Oferta}

Para la estimación de la oferta de las energías renovables se utilizaron fuentes de información nacionales e internacionales.

\subsection{Determinación de las alternativas energéticas}

Dado que las aplicaciones tecnológicas de las energías renovables se están desarrollando rápidamente se podrían elegir múltiples alternativas. Sin embargo, está claro que un estudio como este haría inviable la planificación. Por lo tanto, en este estudio se han tenido en cuenta los diferentes tipos de energía renovable que se están utilizando actualmente y son potencialmente utilizables en el área de estudio. Según esto, las alternativas elegidas fueron: 
- Solar Fotovoltaica (PV)

- Solar Térmica (ST)

- Energía Eólica Baja Potencia (EOL)

- Leña (LEÑ)

- Carbón vegetal (CV)

- Biocombustibles líquidos (BL)

- Mini Hidráulica $(\mathrm{MH})$

- Geotérmica (GEO)

A continuación, se describen cada una de las alternativas elegidas.

\subsubsection{Solar Fotovoltaica (PV)}

Se consideran equipos domiciliarios con potencias de hasta $320 \mathrm{~W}$. Asimismo, parques solares de hasta $2 \mathrm{MW}$.

\subsubsection{Solar Térmica (ST)}

Se considera energía solar de baja temperatura $\left(\mathrm{T}<90^{\circ} \mathrm{C}\right)$, principalmente para uso domiciliario.

\subsubsection{Energía Eólica Baja Potencia (EOL)}

Se consideran aerogeneradores de hasta $50 \mathrm{~kW}$. Para uso en el medio rural y complemento para viviendas.

\subsubsection{Leñas (LEÑ)}

Comprende el uso madera proveniente de árboles autóctonos y de residuos forestales, para uso doméstico.

\subsubsection{Carbón Vegetal (CV)}

Comprende el uso de la leña procesada para uso doméstico e industrial.

\subsubsection{Biocombustibles líquidos (BL)}

Se considera el uso de Gasohol y Biodiesel de origen autóctono. 


\subsubsection{Mini Hidráulica (MH)}

Comprende minicentrales hidroeléctricas de 100 a $1000 \mathrm{~kW}$, instaladas localmente.

\subsubsection{Geotérmica (GEO)}

Por la particularidad del recurso en la zona, se considera el uso directo de esta energía a nivel domiciliario.

En la Tabla 4-1 se presentan alternativas energéticas consideradas, su descripción y el uso de las mismas.

Tabla 4-1. Resumen de las alternativas energéticas consideradas en el modelo SEPLAN.

\begin{tabular}{|c|c|c|c|}
\hline Alternativa & Símbolo & Descripción & Uso \\
\hline Solar Fotovoltaica & PV & $\begin{array}{l}\text { Equipos domiciliarios con potencias de } \\
\text { hasta } 320 \mathrm{~W} \text {. Asimismo, parques solares de } \\
\text { hasta } 8 \mathrm{MW} .\end{array}$ & Eléctrico \\
\hline Solar Térmica & ST & $\begin{array}{l}\text { Energía solar de baja temperatura }(\mathrm{T}< \\
\left.90^{\circ} \mathrm{C}\right) \text {, principalmente para uso domiciliario. }\end{array}$ & Térmico \\
\hline Eólica Baja Potencia & EOL & $\begin{array}{l}\text { Aerogeneradores de hasta } 50 \mathrm{~kW} \text {. Uso en el } \\
\text { medio rural y complemento para viviendas } \\
\text { urbanas. }\end{array}$ & Eléctrico \\
\hline Leñas & LEÑ & $\begin{array}{l}\text { Uso de madera proveniente de residuos } \\
\text { forestales, para uso doméstico con cocina } \\
\text { mejoradas. }\end{array}$ & Térmico \\
\hline Carbón Vegetal & $\mathrm{CV}$ & $\begin{array}{l}\text { Uso de leña procesada para uso doméstico e } \\
\text { industrial. }\end{array}$ & Térmico \\
\hline $\begin{array}{l}\text { Biocombustibles } \\
\text { Líquidos }\end{array}$ & BL & Gasohol y Biodiesel de origen autóctono. & Transporte \\
\hline Mini Hidráulica & MH & $\begin{array}{l}\text { Minicentrales hidroeléctricas de } 100 \text { a } 1000 \\
\mathrm{~kW} \text {, instaladas localmente. }\end{array}$ & Eléctrico \\
\hline Geotérmica & GEO & Uso directo a nivel domiciliario. & Térmico \\
\hline
\end{tabular}

Lo especifico de la selección de las alternativas energéticas en el modelo, no resta su potencial para aplicarlo a otras regiones con características específicas.

\subsection{Funciones Objetivo}


Los modelos de planificación deben ser sencillos y fáciles de operar. Para ello, en el modelo SEMA se establece que todas las funciones sean lineales. En el modelo SEPLAN se conserva la estructura lineal de las funciones, las cuales se describen a continuación.

\subsubsection{F1: Maximización de la sustitución de las energías fósiles por energías renovables.}

Esta función objetivo maximiza la oferta de energías renovables, priorizando el uso de unas fuentes de energías renovables frente a otras. Existen unas características intrínsecas de la sociedad donde se pretenden introducir las fuentes renovables, que condicionan su grado de implantación. Por otro lado, el grado de desarrollo tecnológico en que se encuentran las energías renovables es distinto. Estos dos hechos condicionan a cada energía renovable, estableciéndose prioridades de unas frente a otras. Para reflejar las diferencias, se le asigna a cada fuente renovable un coeficiente que la compare frente a las demás, dando mayor importancia a aquellas energías renovables con mayor futuro y penalizando las restantes según los criterios sociales, económicos y técnicos oportunos (García, 2004).

La función tiene la siguiente estructura matemática:

$$
F_{1}\left(x_{11}, x_{12}, \ldots, x_{i j}, \ldots, x_{n m}\right)=A_{11} x_{11}+A_{12} x_{12}+\ldots+A_{n m} x_{n m}
$$

Dónde:

$x_{11}, x_{12}, \ldots x_{i j}, \ldots, x_{n m}$ muestran la cantidad de energía sustituida por los diferentes tipos de energías renovables.

$A_{11}, A_{12}, \ldots A_{n m}$ son los coeficientes asignados a cada aplicación "j" de cada energía renovable "i". Estos coeficientes son adimensionales.

Para calcular los coeficientes, se utilizó una técnica de análisis vectorial, denominada Método de las Precedencias, vista en el apartado 3.1.6.3. De acuerdo con este método, se establecieron niveles de comparación para evaluar cada alternativa energética y compararlas entre sí. Según esto, se obtuvieron las alternativas prioritarias. Los niveles de comparación considerados fueron los que utiliza el modelo SEMA para esta función. En la Tabla 4-2 se presenta la descripción de cada uno de dichos niveles. 
Tabla 4-2. Niveles de comparación de la función objetivo $\mathrm{F}_{1}$, descripción y evaluación numérica considerada.

\begin{tabular}{|c|c|c|c|}
\hline Nivel & Criterio & Descripción & Evaluación \\
\hline \multirow{6}{*}{ Tecnología } & Demanda Tecnológica & Demanda de la tecnología por parte de la población. & $\begin{array}{l}\text { La experiencia adquirida por los proveedores locales muestra el impacto } \\
\text { de las tecnologías en la población. La escala utilizada oscila entre } 5 \text { (alta } \\
\text { demanda tecnológica) y } 1 \text { (baja demanda tecnológica). }\end{array}$ \\
\hline & Capacidad de investigación & Posibilidad de investigación local sobre la tecnología. & $\begin{array}{l}\text { Líneas de investigación en centros de investigación locales definen el } \\
\text { desarrollo futuro de la tecnología. La escala utilizada varía de } 5 \\
\text { (investigación de alto potencial) a } 1 \text { (baja investigación de potencial). }\end{array}$ \\
\hline & $\begin{array}{l}\text { Ampliación de la } \\
\text { experiencia }\end{array}$ & $\begin{array}{l}\text { Instalaciones, prácticas existentes y posibilidad de ampliación de } \\
\text { las mismas para aumentar el know how de las tecnologías. }\end{array}$ & $\begin{array}{l}\text { Número de proyectos e instalaciones realizadas relacionadas con la } \\
\text { tecnología. La escala utilizada va de } 1 \text { a } 5 \text {. Un alto know-how se valora } \\
\text { con } 5 \text { y un bajo know-how se valora con } 1 \text {. }\end{array}$ \\
\hline & Mejora de la tecnología & $\begin{array}{l}\text { Actividad desarrollada para la puesta a punto de una segunda } \\
\text { generación de equipos, prácticamente de fabricación nacional o } \\
\text { regional. }\end{array}$ & $\begin{array}{l}\text { La escala utilizada va de } 1 \text { a } 5 \text {. Una gran posibilidad de mejora } \\
\text { tecnológica se valoró con } 5 \text {. Una baja posibilidad de mejora tecnológica } \\
\text { se valoró con } 1 \text {. }\end{array}$ \\
\hline & Nivel tecnológico adquirido & Conocimientos actuales para la aplicación de la alternativa. & $\begin{array}{l}\text { La escala utilizada oscila entre } 5 \text { (alto nivel tecnológico alcanzado) y } 1 \\
\text { (bajo nivel tecnológico alcanzado). }\end{array}$ \\
\hline & Tiempo de operación & $\begin{array}{l}\text { Tiempo que ha de transcurrir para que una alternativa esté puesta a } \\
\text { punto en el mercado energético. }\end{array}$ & $\begin{array}{l}\text { La escala utilizada oscila entre } 5 \text { (bajo tiempo de funcionamiento) y } 1 \\
\text { (alto tiempo de funcionamiento). }\end{array}$ \\
\hline \multirow{4}{*}{ Aplicación } & $\begin{array}{l}\text { Facilidad de } \\
\text { implementación }\end{array}$ & $\begin{array}{l}\text { Falta de los obstáculos que aparecen al realizar una instalación. } \\
\text { Puesta en marcha en un tiempo adecuado. }\end{array}$ & $\begin{array}{l}\text { La escala utilizada va de } 1 \text { a } 5 \text {. Una implementación difícil se valoró con } \\
1 \text { y una implementación fácil se valoró con } 5 \text {. }\end{array}$ \\
\hline & Capacidad de integración & $\begin{array}{l}\text { Facilidad de una alternativa para conectarse con equipos y medios } \\
\text { ya existentes. }\end{array}$ & $\begin{array}{l}\text { La escala utilizada oscila entre } 5 \text { (posibilidad de integración alta) y } 1 \\
\text { (posibilidad de integración baja). }\end{array}$ \\
\hline & Demanda potencial & $\begin{array}{l}\text { Existencia de un mercado potencial que demande y consuma esa } \\
\text { alternativa. }\end{array}$ & $\begin{array}{l}\text { La escala utilizada oscila entre } 5 \text { (gran demanda potencial) y } 1 \text { (baja } \\
\text { demanda potencial). }\end{array}$ \\
\hline & Disponibilidad de oferta & $\begin{array}{l}\text { Existencia de empresas dispuestas a realizar la comercialización de } \\
\text { la oferta en el mercado energético. }\end{array}$ & $\begin{array}{l}\text { La escala utilizada va de } 1 \text { a } 5 \text {. Una gran disponibilidad de suministro se } \\
\text { valoró con } 5 \text { y una baja disponibilidad de suministro se valoró con } 1 \text {. }\end{array}$ \\
\hline \multirow{4}{*}{$\begin{array}{l}\text { Planificación } \\
\text { Energética }\end{array}$} & $\begin{array}{l}\text { Interés de política } \\
\text { energética }\end{array}$ & $\begin{array}{l}\text { Cuantificación de la prioridad en la economía energética, que los } \\
\text { órganos de gobierno nacional dan a la alternativa. }\end{array}$ & $\begin{array}{l}\text { La escala utilizada oscila entre } 5 \text { (alto interés político) y } 1 \text { (bajo interés } \\
\text { político). }\end{array}$ \\
\hline & Precio actual & Comparación con los precios de las energías clásicas. & $\begin{array}{l}\text { La escala utilizada va de } 1 \text { a } 5 \text {. Un precio actual alto se valoró con } 1 \text { y un } \\
\text { precio actual bajo se valoró con } 5 \text {. }\end{array}$ \\
\hline & Demanda social & Interés de los agentes sociales en la utilización de la alternativa. & $\begin{array}{l}\text { La escala utilizada oscila entre } 5 \text { (alta demanda social) y } 1 \text { (baja demanda } \\
\text { social). }\end{array}$ \\
\hline & Legislación prevista & $\begin{array}{l}\text { Aspectos legislativos que impidan o fomenten el desarrollo de la } \\
\text { alternativa. }\end{array}$ & $\begin{array}{l}\text { La escala utilizada va de } 1 \text { a } 5 \text {. Una legislación prevista positiva se valoró } \\
\text { con } 5 \text { y una legislación prevista negativa se valoró con } 1 .\end{array}$ \\
\hline Ambiental & Aspectos ambientales & $\begin{array}{l}\text { Evaluación general de la incidencia medioambiental de la } \\
\text { alternativa. }\end{array}$ & $\begin{array}{l}\text { La escala utilizada oscila entre } 5 \text { (bajo impacto ambiental) y } 1 \text { (alto } \\
\text { impacto ambiental). }\end{array}$ \\
\hline
\end{tabular}


La valoración de las alternativas se realizó a través de la consulta a expertos, los cuales asignan valores del 1 al 5 para cada alternativa (Tabla 4-2), según los niveles de comparación, obteniéndose para cada una, un vector de dos componentes $\mathrm{N}_{\mathrm{i}}$ y $\mathrm{M}_{\mathrm{i}}$, donde:

$$
\begin{aligned}
& N_{i j}=\prod_{i=1}^{n} a_{i j} \\
& M_{i j}=\prod_{i=1}^{n}\left(6-a_{i j}\right)
\end{aligned}
$$

Una vez ordenadas las alternativas según los valores de $\mathrm{N}_{\mathrm{i}}$ y $\mathrm{M}_{\mathrm{i}}$, se dividieron en cuatro grupos correspondientes a cuatro rangos de igual magnitud entre el mayor y el menor valor, asignando a cada uno los siguientes valores, de mayor a menor importancia:

Grupo 1: Valor de coeficiente $\mathrm{A}_{\mathrm{i}}=2,5$

Grupo 2: Valor de Coeficiente $A_{i}=2,0$

Grupo 3: Valor de Coeficiente $A_{i}=1,5$

Grupo 4: Valor de Coeficiente $A_{i}=1,0$

Estos coeficientes fueron utilizados en la función objetivo.

\subsection{2 $\mathrm{F}_{2}, \mathrm{~F}_{3}$ y $\mathrm{F}_{4}$ : Maximización de las emisiones evitadas de $\mathrm{CO}_{2}$, $\mathrm{NO}_{x}$ y $\mathrm{SO}_{2}$, respectivamente.}

Los GEI son la principal causa del cambio climático en el mundo, por ello, en la actualidad, todos los países promueven nuevos modelos energéticos bajos en carbono. Las emisiones de $\mathrm{CO}_{2}, \mathrm{NO}_{\mathrm{X}}$ y $\mathrm{SO}_{2}$ son de especial relevancia en los países en vías de desarrollo ya que provienen directamente del uso de combustibles fósiles para el transporte, lo cual se acentúa todavía más, considerando la antigüedad del parque automotor en dichas regiones.

Las funciones para la maximización de emisiones evitadas tienen la siguiente estructura:

$$
\begin{aligned}
& F_{2}\left(x_{11}, x_{12}, \ldots, x_{i j}, \ldots, x_{n m}\right)=B_{11} x_{11}+B_{12} x_{12}+\ldots+B_{n m} x_{n m} \\
& F_{3}\left(x_{11}, x_{12}, \ldots, x_{i j}, \ldots, x_{n m}\right)=C_{11} x_{11}+C_{12} x_{12}+\ldots+C_{n m} x_{n m} \\
& F_{4}\left(x_{11}, x_{12}, \ldots, x_{i j}, \ldots, x_{n m}\right)=D_{11} x_{11}+D_{12} x_{12}+\ldots+D_{n m} x_{n m}
\end{aligned}
$$


Dónde:

$x_{11}, x_{12}, \ldots x_{i j}, \ldots, x_{n m}$ muestran la cantidad de energía sustituida por los diferentes tipos de energías renovables.

$B_{11}, B_{12}, \ldots, B_{n m} ; C_{11}, C_{12}, \ldots, C_{n m} ; D_{11}, D_{12}, \ldots, D_{n m}$ son los coeficientes de reducción de las emisiones.

$B_{i j}, C_{i j}, D_{i j}=$ Emisión equivalente de $\mathrm{CO}_{2}, \mathrm{NO}_{\mathrm{x}} \mathrm{y} \mathrm{SO}_{2}$, respectivamente, evitada por unidad energética, al utilizar la fuente de energía renovable $\mathrm{i}$.

Los coeficientes B, C y D fueron obtenidos de organismos nacionales oficiales. A diferencia de los obtenidos para la $\mathrm{F}_{1}$, estos coeficientes fueron valores cuantitativos.

\subsubsection{F5: Minimización de los costes.}

Los costes de las energías renovables en el sector de la energía se suelen expresar de dos maneras: por unidad de capacidad instalada y por unidad de electricidad generada. Los costes por unidad de capacidad instalada se denominan costes de capital, también llamados "overnight costs", y se expresan en dólares por kilovatio (USD/kW). Se calculan dividiendo la inversión total de la instalación entre su capacidad total. El LCOE (Levelised Cost of Electricity) o coste nivelado de la electricidad, representa el coste por unidad de electricidad producida y se expresa en dólares por megavatio-hora (USD/MWh) (International Energy Agency, 2016).

En el presente estudio se ha tomado en cuenta el LCOE, pues se considera que podría representar con mayor exactitud el coste global de los proyectos al incluir en su cálculo lo siguiente:

- Costes de capital o gastos iniciales adelantados.

- Servicio de la deuda y retorno del capital invertido (representado por el promedio ponderado del coste de capital).

- Costes de operación y mantenimiento.

- Los costes de combustible y los costes asociados por el dióxido de carbono u otras emisiones generadas (si tuvieran un precio en el mercado).

- Costos de desinstalación (si corresponde).

Según esto, la función lineal de costes es expresada de la siguiente manera: 


$$
F_{5}\left(x_{11}, x_{12}, \ldots, x_{i j}, \ldots, x_{n m}\right)=E_{11} x_{11}+E_{12} x_{12}+\ldots+E_{n m} x_{n m}
$$

Dónde:

$x_{11}, x_{12}, \ldots x_{i j}, \ldots, x_{n m}$ muestran la cantidad de energía sustituida por los diferentes tipos de energías renovables.

$E_{11}, E_{12}, \ldots, E_{n m}$ son los coeficientes de costes.

$E_{i j}=\mathrm{LCOE}(\mathrm{USD} / \mathrm{ktoe})$ de cada alternativa energética.

$\forall E_{i j} \geq 0$

Al igual que los coeficientes $B_{i j}, C_{i j} \mathrm{y} D_{i j}$, los coeficientes $E_{i j}$ fueron obtenidos de fuentes oficiales.

\subsubsection{F6: Maximización del uso de alternativas energéticas renovables para el suministro sostenible de energía en comunidades rurales aisladas.}

Esta función considera el suministro de energía sostenible en las CRA. Se ha desarrollado tomando como base la experiencia de ACCIONA Microenergía Perú. Esta microempresa social es el principal proveedor de energía solar fotovoltaica distribuida en Perú. Actualmente su intervención se concentra en la provincia de Cajamarca, región ubicada al norte del país.

Según el último Censo de Población y Vivienda del Perú realizado en 2007, el $25,9 \%$ de los hogares no tenía energía eléctrica. Este porcentaje alcanza su mayor valor en la región de Cajamarca (Tabla 4-3), donde el 59,8\% de las viviendas no tenían ese servicio (Fondo Multilateral de Inversiones, 2014). El alto grado de dispersión de las viviendas en la zona rural hace que la ampliación de la red sea económicamente inviable, situación que motiva la búsqueda de nuevas tecnologías energéticas (Yadoo \& Cruickshank, 2012).

Tabla 4-3. Coeficientes de electrificación en Perú por región (United Nations Development Programme, 2009).

\begin{tabular}{lclc}
\hline Regiones & $\begin{array}{c}\text { Coeficiente de } \\
\text { región }\end{array}$ & $\begin{array}{c}\text { Provincia con } \\
\text { electrificación más baja }\end{array}$ & $\begin{array}{c}\text { Coeficiente } \\
\text { de provincia }\end{array}$ \\
\hline Caiamarca & 0,4 & San Pablo & 0,19 \\
Huánuco & 0,4 & Puerto Inca & 0,11 \\
Amazonas & 0,5 & Condorcanqui & 0,10 \\
Ayacucho & 0,5 & Vilcas Huamán & 0,19 \\
Huancavelica & 0,6 & Castrovirreyna & 0,37 \\
Apurímac & 0,6 & Cotabambas & 0,31 \\
\hline
\end{tabular}




\begin{tabular}{lclc}
\hline \multicolumn{1}{c}{ Regiones } & $\begin{array}{c}\text { Coeficiente de } \\
\text { región }\end{array}$ & $\begin{array}{c}\text { Provincia con } \\
\text { electrificación más baja }\end{array}$ & $\begin{array}{c}\text { Coeficiente } \\
\text { de provincia }\end{array}$ \\
\hline Puno & 0,6 & Lampa & 0,38 \\
San Martín & 0,6 & El Dorado & 0,36 \\
Loreto & 0,6 & Datem del Marañón & 0,27 \\
Cusco & 0,6 & Chumbivilcas & 0,19 \\
Ucayali & 0,7 & Atalaya & 0,32 \\
Piura & 0,7 & Ayabaca & 0,27 \\
Madre de Dios & 0,7 & Manu & 0,45 \\
Pasco & 0,7 & Oxapampa & 0,51 \\
La Libertad & 0,7 & Julcán & 0,20 \\
Ancash & 0,7 & C, F, Fitzcarrald & 0,31 \\
Junín & 0,7 & Satipo & 0,39 \\
Lambayeque & 0,8 & Lambayeque & 0,50 \\
Ica & 0,8 & Pisco & 0,65 \\
Moquegua & 0,8 & General Sánchez Cerro & 0,56 \\
Tumbes & 0,8 & Zarumilla & 0,76 \\
Tacna & 0,8 & Tarata & 0,63 \\
Arequipa & 0,8 & La Unión & 0,41 \\
Lima & 0,9 & Canta & 0,59 \\
\hline
\end{tabular}

El Plan Nacional de Electrificación Rural 2013-2022, elaborado por el Ministerio de Energía y Minas de Perú (Ministerio de Energía y Minas, 2012), en concordancia con el Plan Universal de Acceso a la Energía, establece una política para el sector con el objetivo de elevar la tasa de electrificación rural del 87\% al 95\% (Figura 4-2). Perú está realizando esfuerzos para proporcionar este acceso a la energía con licitaciones dirigidas a instalar sistemas solares domésticos, mini-redes con energía hidroeléctrica, solar y eólica, y ampliación de la red (Lillo, et al., 2015).

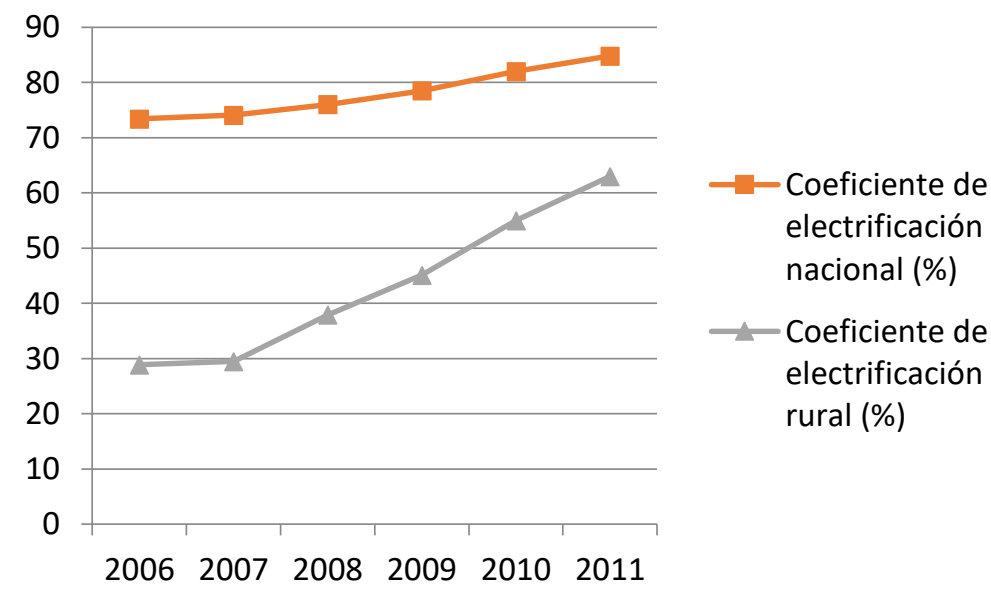

Figura 4-2. Coeficientes de electrificación nacional en Perú al 2011. Fuente: MINEM (2012).

Sin embargo, tal y como se mencionaba en el capítulo introductorio, la experiencia ha demostrado que las soluciones tecnológicas no son suficientes para garantizar el acceso 
a la energía en las CRA. Es también fundamental diseñar un modelo de gestión que considere los aspectos sociales y culturales de las CRA para lograr la sostenibilidad de los proyectos en este tipo de comunidades.

La Figura 4-3 muestra un Árbol de Medios y Fines elaborado por ACCIONA Microenergía Perú. Este esquema conceptual incorpora los criterios mencionados anteriormente y podría proporcionar las claves para elegir la alternativa más adecuada, esto es, la alternativa que suministre energía a las CRA de manera integral, fiable y eficiente.

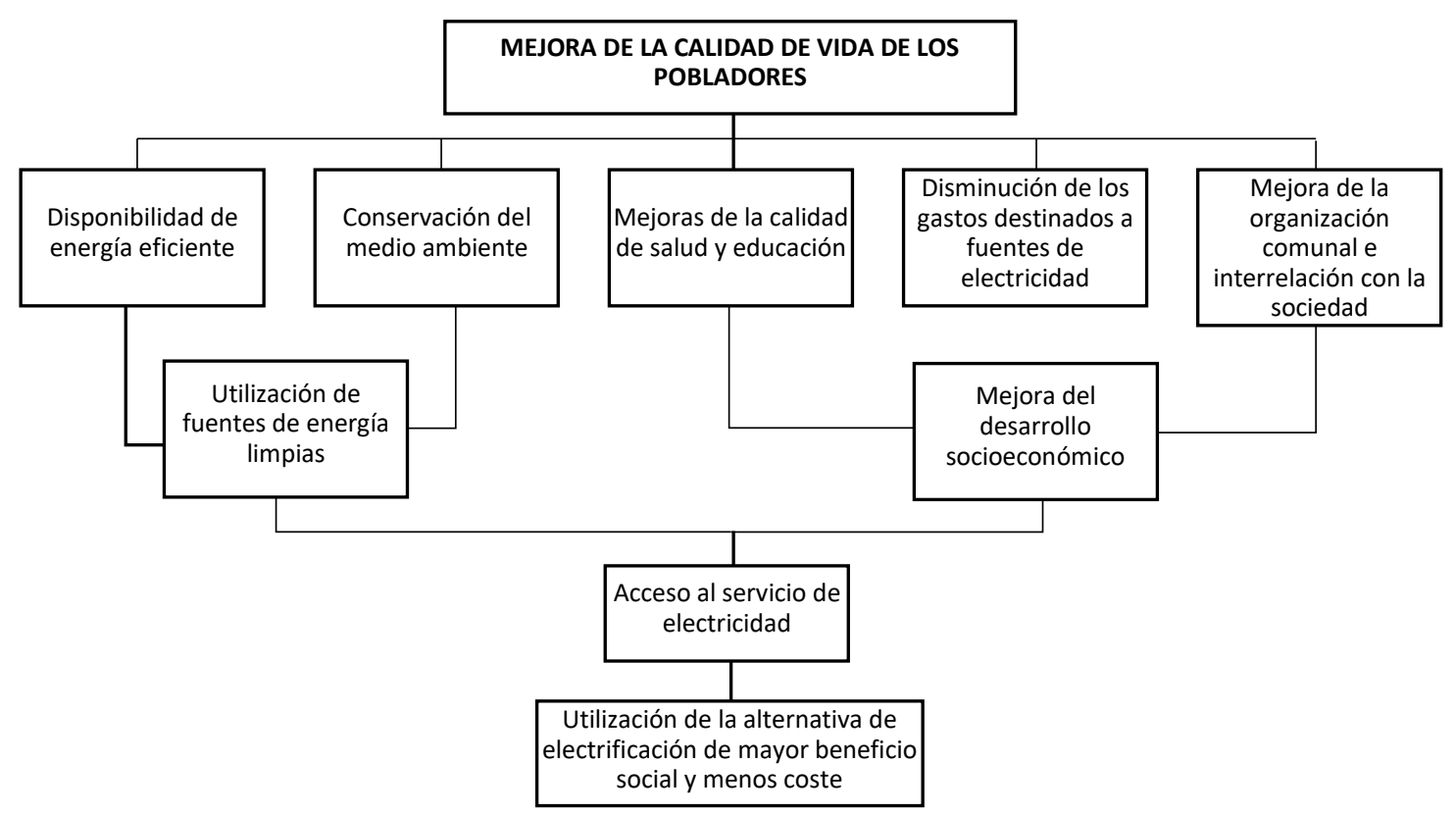

Figura 4-3. Árbol de Medios y Fines en proyectos de electrificación rural (ACCIONA Microenergía Perú, 2011)

De acuerdo a lo mencionado, la ecuación obtenida para $\mathrm{F}_{6}$ fue la siguiente:

$$
F_{6}\left(x_{11}, x_{12}, \ldots, x_{i j}, \ldots, x_{n m}\right)=G_{11} x_{11}+G_{12} x_{12}+\ldots+G_{n m} x_{n m}
$$

Dónde:

$x_{11}, x_{12}, \ldots x_{i j}, \ldots, x_{n m}$ muestran la cantidad de energía sustituida por los diferentes tipos de energías renovables.

Los coeficientes $G_{11}, G_{12}, \ldots G_{n m}$ se obtuvieron siguiendo el mismo método que para $F_{1}$. Los criterios y los niveles de comparación se determinaron según la experiencia de los proveedores locales de energía en las CRA y la consulta a expertos. En la Tabla 44 se indica la descripción de los criterio tomados en cuenta en cada nivel de comparación, así como la evaluación numérica considerada en cada caso. 
Tabla 4-4. Niveles de comparación de la función objetivo $\mathrm{F}_{6}$, descripción y evaluación numérica considerada.

\begin{tabular}{|c|c|c|c|}
\hline $\begin{array}{c}\text { Niveles de } \\
\text { comparación }\end{array}$ & Criterios & Descripción & Evaluación \\
\hline \multirow{6}{*}{ Tecnológico } & Larga vida útil & $\begin{array}{l}\text { Durabilidad de los equipos. Influye directamente en la economía de } \\
\text { los proyectos. }\end{array}$ & $\begin{array}{l}\text { La escala utilizada oscila entre } 5 \text { (larga vida útil) y } 1 \text { (vida útil } \\
\text { corta). }\end{array}$ \\
\hline & Requiere batería & $\begin{array}{l}\text { La tecnología requiere el almacenamiento de energía a través de } \\
\text { baterías. Se ha considerado favorable prescindir de las mismas. }\end{array}$ & $\begin{array}{l}\text { La escala utilizada oscila entre } 5 \text { (no se requiere batería) y } 1 \text { (se } \\
\text { requiere batería). }\end{array}$ \\
\hline & Salida CA & $\begin{array}{l}\text { La salida a corriente alterna (CA), se considera favorable en las } \\
\text { tecnologías a evaluar, ya que permiten mayor amplitud en el uso de } \\
\text { artefactos. }\end{array}$ & $\begin{array}{l}\text { La escala utilizada va de } 1 \text { a } 5 \text {. Una gran posibilidad de usar esta } \\
\text { característica se valoró con } 5 \text {, mientras que una baja posibilidad } \\
\text { se valoró con } 1 \text {. }\end{array}$ \\
\hline & Modularidad del sistema & $\begin{array}{l}\text { Ventaja que tienen algunas tecnologías para permitir ampliar su } \\
\text { capacidad de generación de energía, a través de conexiones en serie o } \\
\text { en paralelo. }\end{array}$ & $\begin{array}{l}\text { La escala utilizada varía de } 5 \text { (alta posibilidad de modularidad) a } \\
1 \text { (baja posibilidad de modularidad). }\end{array}$ \\
\hline & Fácil hibridación & $\begin{array}{l}\text { Asegura la continuidad de la producción energética de los equipos } \\
\text { durante el día y la noche. }\end{array}$ & $\begin{array}{l}\text { La escala utilizada va de } 1 \text { a } 5 \text {. Los sistemas con hibridación fácil } \\
\text { se valoraron con } 5 \text {, mientras que los sistemas con hibridación } \\
\text { difícil se valoraron con } 1 \text {. }\end{array}$ \\
\hline & $\begin{array}{l}\text { Requiere importar } \\
\text { equipos }\end{array}$ & $\begin{array}{l}\text { Necesidad de las tecnologías de importar el total o una parte de sus } \\
\text { componentes. Tienen ventaja aquellas que lo requieran } \\
\text { mínimamente. }\end{array}$ & $\begin{array}{l}\text { La escala utilizada va de } 1 \text { a } 5 \text {. Los sistemas que no requieren } \\
\text { importar partes o pocas se valoraron con } 5 \text {. Los sistemas que } \\
\text { requieren importar todas sus partes se valoraron con } 1 .\end{array}$ \\
\hline \multirow{3}{*}{ Económico } & Bajo LCOE & $\begin{array}{l}\text { LCOE (Levelized Cost of Electricity) es el coste para generar } 1 \text { tep } \\
\text { durante la vida útil del equipo. }\end{array}$ & $\begin{array}{l}\text { La escala utilizada va de } 1 \text { a } 5 \text {. Las tecnologías con un bajo } \\
\text { LCOE se valoraron con } 5 \text {. Las tecnologías con un alto LCOE se } \\
\text { valoraron con } 1 .\end{array}$ \\
\hline & $\begin{array}{l}\text { Bajo coste de } \\
\text { mantenimiento }\end{array}$ & $\begin{array}{l}\text { En zonas aisladas los desplazamientos pueden incrementar estos } \\
\text { costes considerablemente. }\end{array}$ & $\begin{array}{l}\text { La escala utilizada oscila entre } 5 \text { (menor costo de mantenimiento) } \\
\text { y } 1 \text { (mayor costo de mantenimiento). }\end{array}$ \\
\hline & Escalable y replicable & $\begin{array}{l}\text { La escalabilidad implica el crecimiento del proyecto con el fin de } \\
\text { aumentar su impacto social en una zona concreta }{ }^{\mathrm{a}} \text {, mientras que } \\
\text { replicabilidad está relacionada con la implantación del proyecto en } \\
\text { un contexto geográfico y social distinto }{ }^{\mathrm{b}} \text {. }\end{array}$ & $\begin{array}{l}\text { La escala utilizada varía de } 1 \text { a } 5 \text {. La alta escalabilidad y } \\
\text { replicabilidad se valoró con } 5 \text {, mientras que la baja escalabilidad } \\
\text { y replicabilidad se valoró con } 1 .\end{array}$ \\
\hline \multirow[t]{2}{*}{ Operación } & Fácil instalación & $\begin{array}{l}\text { Aumenta la eficiencia del tiempo durante la ejecución de los } \\
\text { proyectos y evita capacitaciones especializadas. }\end{array}$ & $\begin{array}{l}\text { La escala usada va de } 1 \text { a } 5 \text {. Los sistemas con fácil instalación se } \\
\text { valoraron con } 5 \text {. Los sistemas con instalación difícil se valoraron } \\
\text { con } 1 .\end{array}$ \\
\hline & Capacitación requerida & $\begin{array}{l}\text { Es el grado de capacitación que requerirán los usuarios para operar } \\
\text { los sistemas. }\end{array}$ & $\begin{array}{l}\text { La escala utilizada varía de } 5 \text { (solo se requiere una capacitación } \\
\text { básica) a } 1 \text { (se requiere una capacitación especializada). }\end{array}$ \\
\hline
\end{tabular}




\begin{tabular}{|c|c|c|c|}
\hline $\begin{array}{c}\text { Niveles de } \\
\text { comparación }\end{array}$ & Criterios & Descripción & Evaluación \\
\hline & Seguridad contra robo & $\begin{array}{l}\text { La ubicación en exterior de algunos equipos y las dimensiones de los } \\
\text { mismos podrían aumentar la probabilidad de robo. }\end{array}$ & $\begin{array}{l}\text { La escala utilizada va de } 1 \text { a } 5 \text {. La alta seguridad contra robos se } \\
\text { valoró con } 5 \text {, mientras que la baja seguridad contra robos se } \\
\text { valoró con } 1 \text {. }\end{array}$ \\
\hline \multirow{4}{*}{ Planificación } & $\begin{array}{l}\text { Aceptación y } \\
\text { participación social }\end{array}$ & $\begin{array}{l}\text { Aceptación y participación de las personas beneficiarias en el } \\
\text { modelo de gestión de los proyectos de electrificación ruralc }\end{array}$ & $\begin{array}{l}\text { La escala utilizada va de } 1 \text { a } 5 \text {. Una alta aceptación y } \\
\text { participación social se valoró con } 5 \text {. Una baja aceptación y } \\
\text { participación social se valoró con } 1 .\end{array}$ \\
\hline & Impacto social directo & Impacto que lleva la nueva tecnología al usuario final. & $\begin{array}{l}\text { La escala utilizada va de } 1 \text { a } 5 \text {. Un alto valor de impacto social } \\
\text { directo fue de } 5 \text {. Un bajo impacto social directo se valoró con } 1 \text {. }\end{array}$ \\
\hline & $\begin{array}{l}\text { Generación de empleo } \\
\text { permanente }\end{array}$ & $\begin{array}{l}\text { Empleo que puede generar el proyecto en la zona en forma } \\
\text { sostenible. }\end{array}$ & $\begin{array}{l}\text { La escala se usó de } 1 \text { a } 5 \text {. La alta generación de empleo } \\
\text { permanente se valoró con } 5 \text {. La baja generación de empleo } \\
\text { permanente se valoró con } 1 .\end{array}$ \\
\hline & Regulación tarifaria & Regulación existente en el país donde se desarrolla la intervención. & $\begin{array}{l}\text { La escala utilizada va de } 1 \text { a } 5 \text {. La existencia de una regulación } \\
\text { tarifaria sólida se valoró con } 5 \text {, la ausencia o la regulación } \\
\text { tarifaria débil se valoró con } 1 .\end{array}$ \\
\hline \multirow{3}{*}{ Ambiental } & Bajo impacto ambiental & $\begin{array}{l}\text { Valoración aplicada a los materiales con los que están elaborados los } \\
\text { equipos tecnológicos. }\end{array}$ & $\begin{array}{l}\text { La escala utilizada oscila entre } 5 \text { (bajo impacto ambiental) y } 1 \\
\text { (alto impacto ambiental). }\end{array}$ \\
\hline & $\begin{array}{l}\text { Baja generación de } \\
\text { residuos }\end{array}$ & Generación de residuos durante la instalación de los equipos. & $\begin{array}{l}\text { La escala utilizada oscila entre } 5 \text { (bajo impacto ambiental) y } 1 \\
\text { (alto impacto ambiental). }\end{array}$ \\
\hline & $\begin{array}{l}\text { Disponibilidad de fuente } \\
\text { energética }\end{array}$ & $\begin{array}{l}\text { Potencial de aprovechamiento de las fuentes energéticas renovables } \\
\text { en la zona de estudio. }\end{array}$ & $\begin{array}{l}\text { La escala utilizada oscila entre } 5 \text { (Alta disponibilidad del recurso } \\
\text { energético) y } 1 \text { (Baja disponibilidad del recurso energético). }\end{array}$ \\
\hline
\end{tabular}

a Fundación ACCIONA Microenergía, Centro de Innovación de Tecnologías para el Desarrollo Humano de la Universidad Politécnica de Madrid: Modelo LC-SFD de ACCIONA Microenergía Perú. Informe de escalabilidad. Madrid, 2015.

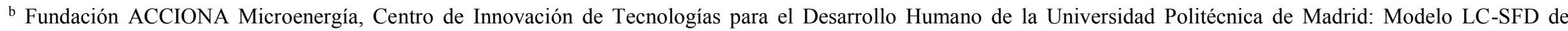
ACCIONA Microenergía Perú. Informe de replicabilidad. Madrid, 2015.

${ }^{\mathrm{c}}$ Según la experiencia de ACCIONA Microenergía Perú, este factor es clave en la sostenibilidad en proyectos de electrificación rural. 


\subsection{Restricciones}

Según García (2004), no se puede pensar en cubrir la demanda de energía de un territorio con fuentes de energía renovables solamente. Las fuentes de energía renovables sólo tienen capacidad para cubrir parte de la demanda energética, debido a problemas técnicos, de mercado, de capacidad de instalación etc. (por ejemplo, la demanda de combustibles líquidos para el transporte). También existen sectores cuyo consumo de energías clásicas debe mantenerse, pues el cambio a energías renovables supondría perjuicios económicos no asumibles por la sociedad.

Por otro lado, existe un potencial real de las energías renovables fijado por su propia naturaleza y a la necesidad de mantener el medio natural, sin dar lugar a una sobreexplotación de los recursos. Se debe tener en cuenta, además, que no todo lo que se produce da lugar a energía aprovechable. Asimismo, se debe considerar la existencia de instalaciones de energía renovable en funcionamiento antes del periodo de planificación, ya que marca la necesidad de poner un límite inferior al aprovechamiento de este tipo de energía. Mientras no esté amortizada la instalación va a seguir funcionando, y por tanto, sustituyendo a una energía clásica (García, 2004).

Según estos criterios, se han considerado las restricciones propuestas por García (2004) en el modelo SEMA, las cuales se agrupan en cuatro tipos según su origen:

\subsubsection{Demanda de energía no eléctrica $\left(S_{j}\right)$ :}

Se trata de la demanda de energía térmica. Fue calculada como: $S_{j}=p_{j}-i_{j}$; donde $p_{j}$ es la energía demandada correspondiente al sector $j$ y $i_{j}$ es la energía obtenida de combustibles fósiles que no puede ser reemplazada por fuentes renovables. La forma de estas inecuaciones es:

$$
\sum_{j=1}^{n} X i j \leq S_{j}
$$

Donde $X_{i j}$ representa la participación energética de las “ $n$ ” fuentes renovables $i$, que pueden abastecer al sector $j$. 


\subsubsection{Demanda de energía eléctrica $\left(S_{e}\right)$ :}

Se ha distinguido la demanda de energía eléctrica de la demanda del resto de vectores energéticos debido a las características especiales del sector eléctrico en la producción, en la distribución y en el abastecimiento de la energía eléctrica a los consumidores. Fue calculada como: $S_{e}=p_{e}-i_{e}$; donde $p_{e}$ es el total de energía eléctrica demandada y $i_{e}$ en la cantidad de energía eléctrica obtenida de combustibles fósiles que no puede ser reemplazada por fuentes renovables. Esta restricción tiene la siguiente forma:

$$
\sum_{i=1}^{l} X i e \leq S_{e}
$$

$X_{i e}$ representa la cantidad de energía sustituible por las " $l$ " fuentes de energí renovable $i$ que producen energía eléctrica.

\subsubsection{Potencial de producción con energías renovables $\left(P_{i}\right)$ :}

Corresponden a la suma de las dos restricciones anteriores, con el fin de fijar el límite máximo (restricción del potencial real) que pueden alcanzar las fuentes de energía renovables. La forma de estas inecuaciones es:

$$
X i e+\sum_{j=1}^{m} X i j \leq P_{i}
$$

Donde $P_{i}$ es el potencial real de aprovechamiento de la fuente de energía renovable $i$ en el área estudiada.

\subsubsection{Sistemas con ER actuales $\left(R_{i}\right)$ :}

Cantidad mínima de energía convencional que puede ser reemplazada por fuentes renovables, ya que las energías renovables han reemplazado esta cantidad. La forma de estas inecuaciones es similar a la restricción anterior, pero en este caso fijando el límite inferior de este tipo de energía: 


$$
X i e+\sum_{j=1}^{m} X i j \geq R_{i}
$$

En la Tabla 4-5 se indica el resumen de las restricciones consideradas.

Tabla 4-5. Restricciones del modelo.

\begin{tabular}{cccc}
\hline $\begin{array}{c}\text { Demanda de } \\
\text { energía no eléctrica }\end{array}$ & $\begin{array}{c}\text { Demanda de } \\
\text { energía eléctrica }\end{array}$ & $\begin{array}{c}\text { Potencial de } \\
\text { producción con ER }\end{array}$ & $\begin{array}{c}\text { Sistemas con ER } \\
\text { actuales }\end{array}$ \\
\hline$\sum_{j=1}^{n} X i j \leq S j$ & $\sum_{i=1}^{l} X i e \leq S e$ & $X i e+\sum_{j=1}^{m} X i j \leq P i$ & $X i e+\sum_{j=1}^{m} X i j \geq R i$ \\
$(9)$ & $(10)$ & $(11)$ & $(12)$ \\
\hline
\end{tabular}

\subsection{Programación Compromiso}

Yu (1973) y Zeleny (1973) utilizaron el concepto de punto ideal como una referencia para los centros decisores. Según estos autores, este punto es uno en el que cada objetivo alcanza su valor óptimo. El punto ideal puede ser representado por el siguiente vector:

$$
F^{*}=\left(F_{1}^{*}, \ldots F_{i}^{*}, \ldots F_{n}^{*}\right)
$$

Donde $F_{i}^{*}$ es el valor óptimo de la función objetivo $F_{i}(x)$ y $x$ cumple con las restricciones del problema.

Teniendo en cuenta que el punto ideal no es alcanzable, los decisores buscarán un punto eficiente lo más cerca posible de éste. Romero (1996) introdujo el concepto de distancia para encontrar el conjunto de soluciones más cercano al punto ideal. El grado de proximidad entre el objetivo $F_{j}(x)$ y su ideal está dado por la distancia $d_{j}$ :

$$
d_{j}=\left[F_{j}^{*}-F_{j}(x)\right]
$$

Dado que los objetivos suelen tener diferentes unidades y para evitar sesgos hacia objetivos con grandes números, esta distancia debe normalizarse:

$$
d_{j}=\frac{\left[F_{j}^{*}-F_{j}(x)\right]}{\left[F_{j}^{*}-F_{* j}\right]}
$$

Donde $F_{* j}$ es el punto anti-ideal para el j-ésimo objetivo. Al hacer una generalización de la distancia euclídea definida como la distancia más corta entre dos puntos, se obtiene una familia de Métricas: 


$$
L_{p}=\left[\sum_{j=1}^{n}\left[\frac{F_{j}^{*}-F_{j}(x)}{F_{j}^{*}-F_{* j}}\right]^{p}\right] 1 / p
$$

El parámetro $p$ representa la métrica que define la familia de funciones de distancia. Si consideramos a $W_{j}$ como los pesos que representan la importancia relativa del j-ésimo objetivo para el tomador de decisiones, la programación compromiso se convierte en el siguiente problema de optimización:

$$
\operatorname{Min} L_{p}=\left[\sum_{j=1}^{n} W_{j}^{p}\left[\frac{F_{j}^{*}-F_{j}(x)}{F_{j}^{*}-F_{* j}}\right]^{p}\right] 1 / p
$$

Zeleny (1973) llama al conjunto limitado por los puntos $\mathrm{L}_{1}$ y $\mathrm{L}_{\infty}$ el conjunto compromiso. Según Romero (1996) L L corresponde al punto de máxima eficiencia y $\mathrm{L}_{\infty}$ es una solución bien equilibrada. En este trabajo se han calculado $\mathrm{L}_{1} \mathrm{y} \mathrm{L}_{\infty}$, llamadas comúnmente distancias de Manhattan y Chebyshev, respectivamente. Asimismo, se han obtenido distancias intermedias $\left(\mathrm{L}_{\mathrm{k}}\right)$ del conjunto de compromiso. Teniendo en cuenta que el modelo SEPLAN tiene seis funciones de objetivos, las distancias mencionadas se muestran en la Tabla 4-6.

\begin{tabular}{|c|c|c|}
\hline $\mathbf{L}_{1}=$ Distancia Manhattan & $\mathbf{L}_{\mathbf{k}}=$ Distancias intermedias & $\begin{array}{c}\mathbf{L}_{\infty}=\text { Distancia } \\
\text { Chebyshev }\end{array}$ \\
\hline$L_{1}=\sum_{j=1}^{6} W j \frac{F_{j}^{*}-F_{j}(x)}{F_{j}^{*}-F_{* j}}$ & $\begin{aligned} L_{k}=\lambda\left[\frac{W_{1} F_{1}(x)}{F_{1}^{*}-F_{* 1}}+\frac{W_{2} F_{2}(x)}{F_{2}^{*}-F_{* 2}} \ldots+\frac{W_{6} F_{6}(x)}{F_{6}^{*}-F_{* 6}}\right] \\
+(1-\lambda) d \\
0 \leq \lambda \leq 1\end{aligned}$ & $L_{\infty}=d$ \\
\hline
\end{tabular}

Tabla 4-6. Distancias normalizadas a ser minimizadas.

Se debe tener en cuenta que $\mathrm{L}_{1}$ se minimiza de acuerdo con las restricciones mencionadas anteriormente. Con el fin de minimizar $\mathrm{L}_{\infty}$ se han tenido en cuenta las mismas restricciones más las siguientes: $W_{1} \frac{F_{1}^{*}-F_{1}(x)}{F_{1}^{*}-F_{* 1}} \leq d$

$$
\begin{aligned}
& W_{2} \frac{F_{2}^{*}-F_{2}(x)}{F_{2}^{*}-F_{* 2}} \leq d \\
& W_{3} \frac{F_{3}^{*}-F_{3}(x)}{F_{3}^{*}-F_{* 3}} \leq d \\
& W_{4} \frac{F_{4}^{*}-F_{4}(x)}{F_{4}^{*}-F_{* 4}} \leq d \\
& W_{5} \frac{F_{5}^{*}-F_{5}(x)}{F_{5}^{*}-F_{* 5}} \leq d
\end{aligned}
$$


$W_{6} \frac{F_{6}^{*}-F_{6}(x)}{F_{6}^{*}-F_{* 6}} \leq d$

$\mathrm{L}_{\mathrm{k}}$ se minimiza de acuerdo con las mismas restricciones indicadas para minimizar $\mathrm{L}_{\infty}$. Estas distancias se obtienen variando los valores de $\lambda$ de 0 a 1 . Para $\lambda=1$ obtendremos el valor $L_{1}$ y para $\lambda=0$ obtendremos el valor $L_{\infty}$ (ver Tabla $4-6$ ).

\subsection{Programación en Matlab®.}

El programa Matlab ${ }^{\circledR}$ fue la herramienta utilizada para el procesamiento de la información y la obtención de las soluciones óptimas. Su versatilidad y adaptabilidad a múltiples aplicaciones, hacen de Matlab ${ }^{\circledR}$ un potente software de programación, ideal para su uso en la presente tesis doctoral. El lenguaje, herramientas y funciones matemáticas integradas permiten a dicho programa, explorar múltiples enfoques y llegar a una solución más rápida que softwares similares como puede ser el programa $\mathrm{R}$, muy utilizado también en el ámbito científico.

La programación se desarrolló en dos etapas. En primer lugar, se optimizaron las funciones objetivo por separado, de esta forma se obtuvieron los valores ideales y anti ideales, valores con los que se construyó la matriz de pagos. En la segunda etapa se obtuvo el conjunto compromiso o conjunto de soluciones eficientes, mediante la minimización de las distancias de la Tabla 4-6.

A continuación, se muestra el código de la programación realizada de manera específica para esta tesis doctoral.

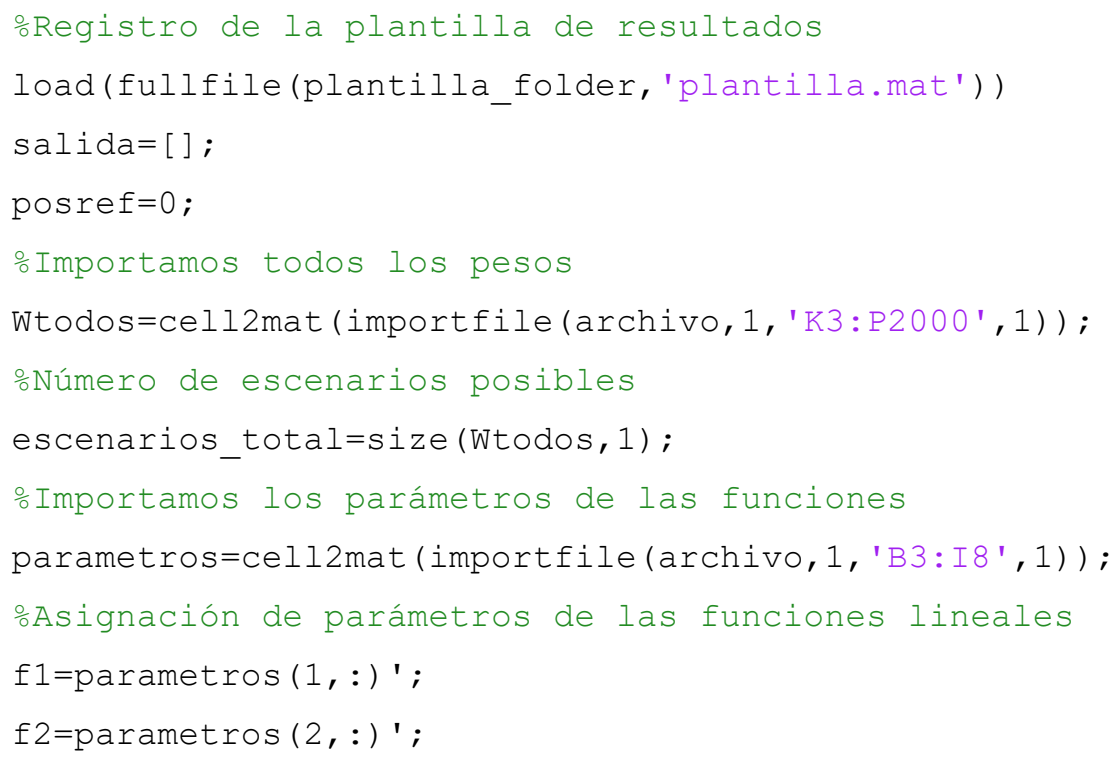




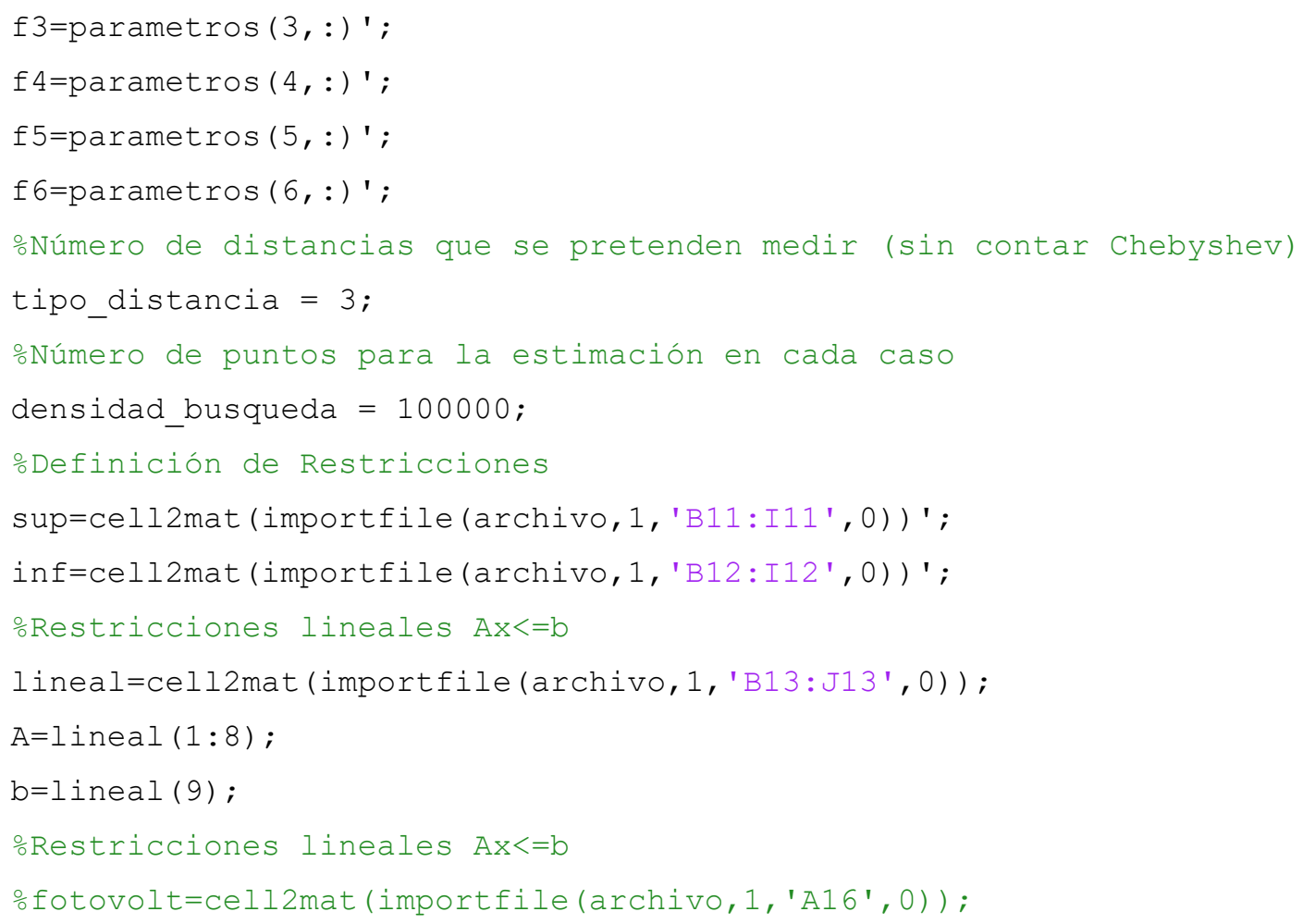

\section{\% PUNTOS OPTIMOS PARA CADA FUNCION OBJETIVO}

oSe cambia de signo SOLO aquellas que se pretenden maximizar $\mathrm{F}=\left[-\mathrm{f} 1^{\prime} ;-\mathrm{f} 2^{\prime} ;-\mathrm{f} 3^{\prime} ;-\mathrm{f} 4^{\prime} ; \mathrm{f5} 5^{\prime} ;-\mathrm{f} 6^{\prime}\right]$;

$\mathrm{F}_{-}=\left[\mathrm{f} 1^{\prime} ; \mathrm{f} 2^{\prime} ; \mathrm{f} 3^{\prime} ; \mathrm{f} 4^{\prime} ; \mathrm{f5} 5^{\prime} ; \mathrm{f} 6^{\prime}\right]$;

oEncuentra el minimo para $\mathrm{F}(\mathrm{x})$ con $\mathrm{Ax}<=\mathrm{b}$ y inf $<=\mathrm{x}<=$ sup $\mathrm{x}=[]$; 


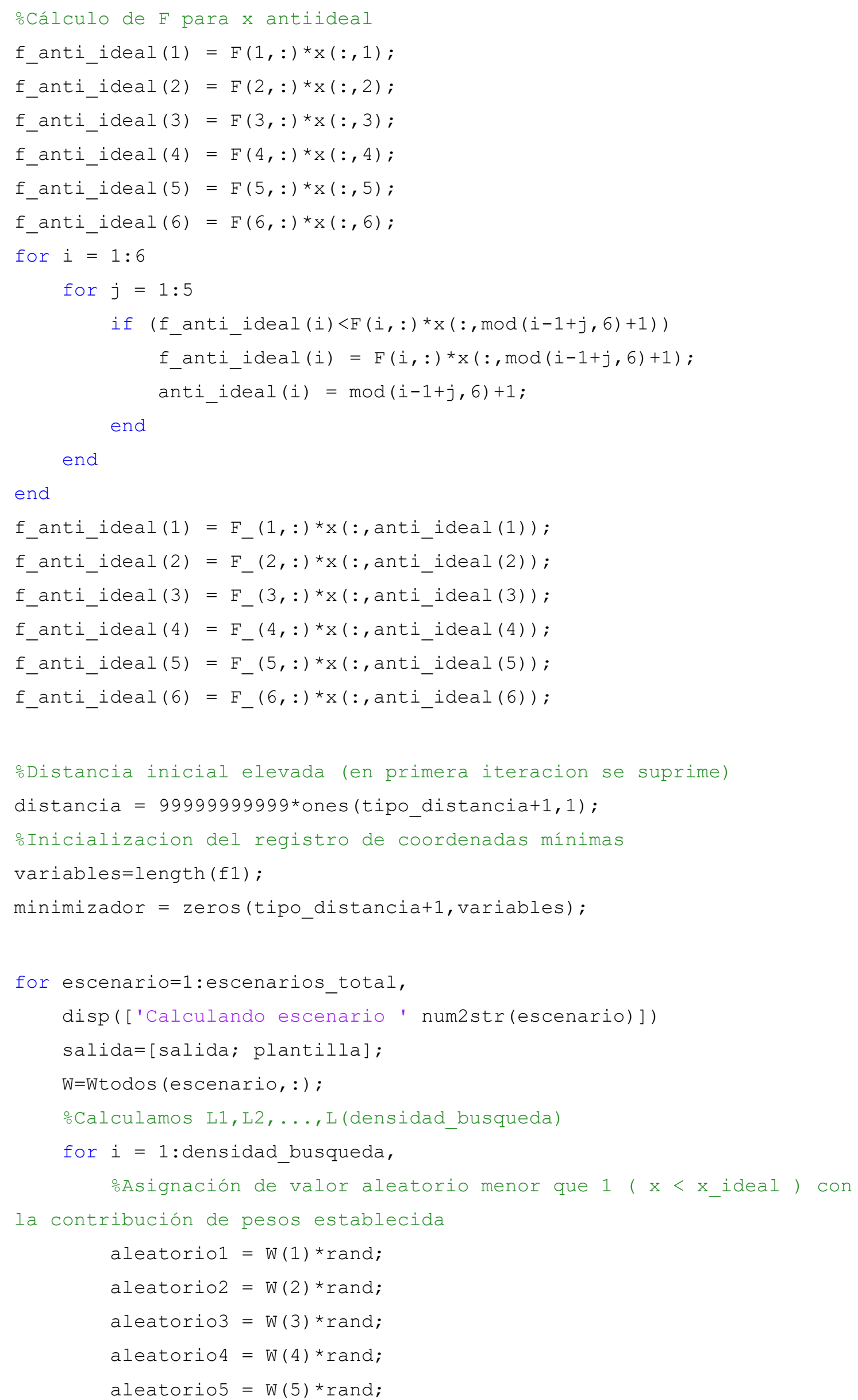




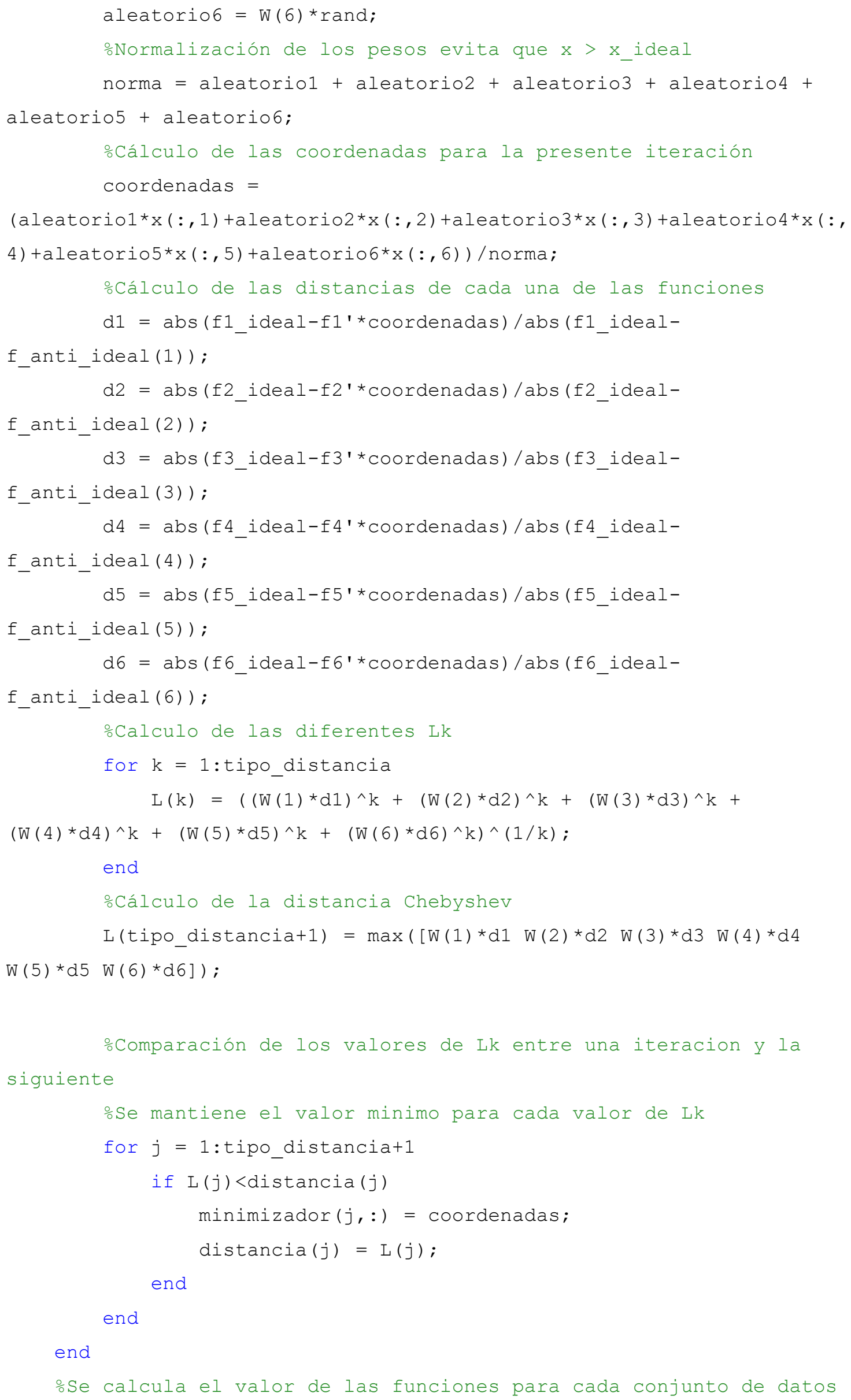


F1value=[];F2value=[];F3value=[];F4value=[];F5value=[];F6value=[];

for $n=1:$ tipo_distanciat1,

Flvalue $=[$ Flvalue minimizador $(\mathrm{n}, \mathrm{:}) * \mathrm{fl}]$;

F2value $=[$ F2value minimizador $(\mathrm{n}, \mathrm{:}) * \mathrm{f} 2]$;

F3value $=[$ F3value minimizador $(\mathrm{n}, \mathrm{:}) * \mathrm{f} 3]$;

F4value $=[$ F4value minimizador $(\mathrm{n}, \mathrm{:}) * \mathrm{f} 4]$;

F5value $=[$ F5value minimizador $(\mathrm{n},:) * f 5]$;

F6value $=[$ F6value minimizador $(\mathrm{n}, \mathrm{:}) * \mathrm{f} 6]$

end

SALIDA DE DATOS

salida (posref 1,2$)=$ num $2 \operatorname{cell}$ (escenario);

salida (posref +3 : posref $+8,1)=\operatorname{num} 2 \operatorname{cell}\left(W^{\prime}\right)$;

salida (posref+3:posref+2+tipo_distancia,2)=num2cell (1:tipo_distancia) ; salida (posref+3+tipo_distancia, 2$)=\{$ 'Chebyshev' $\}$;

salida (posref+3: posref+3+tipo distancia, 3)=num2cell (distancia) ;

salida (posref+3:posref+3+tipo_distancia,4:11)=num2cell (minimizador) ; salida (posref+3:posref+3+tipo_distancia, 12)=num2cell (F1value') ; salida (posref+3:posref+3+tipo_distancia, 13)=num2cell (F2value') ; salida (posref+3:posref+3+tipo_distancia, 14)=num2cell (F3value') ; salida (posref+3:posref+3+tipo_distancia, 15)=num2cell (F4value') ; salida (posref+3:posref+3+tipo_distancia, 16)=num2cell (F5value') ; salida (posref+3:posref+3+tipo_distancia, 17) =num2cell (F6value') ; posref $=$ posref +9 ;

end

oExportación de los datos

rango=['A1:Q' num2str(size(salida,1))]; Rango de salida de los datos xlswrite (archivo, salida, 2, rango)

oAbrir el archivo de salida

winopen (archivo)

disp('Cálculo finalizado') 


\section{CAPÍTULO V}

\section{APLICACIÓN DEL MODELO SEPLAN}

En el presente Capítulo se describe la aplicación del modelo SEPLAN para el caso concreto de la provincia de Cajamarca, Perú.

\subsection{Descripción de la zona de estudio. Provincia de Cajamarca.}

La provincia de Cajamarca fue elegida como zona de aplicación del modelo SEPLAN por los siguientes motivos:

- Es una de las regiones con más población rural de Perú $(70 \%)^{3}$.

- El alto grado de dispersión de las viviendas en la zona rural.

- Presenta el índice electrificación más bajo del país ${ }^{4}$.

- Según informe del INEI del 2016, la pobreza extrema (más de 19\%) es cinco veces mayor al promedio nacional $(3,8 \%)$.

- Presenta una oportunidad de sustitución de combustibles fósiles por energías renovables en la industria minera, sector productivo más importante de la región.

- Experiencia de organizaciones sin fines de lucro en la planificación energética rural.

\subsubsection{Ubicación geográfica}

La provincia de Cajamarca se ubica en el departamento del mismo nombre. El departamento de Cajamarca está situado en la zona norte de Perú, cubre una superficie de $33.318 \mathrm{~km}^{2}$, que representa el 2,6 por ciento del territorio nacional. Limita por el norte con la República del Ecuador, por el este con el departamento de Amazonas, por el sur con La Libertad y por el oeste con Lambayeque y Piura. Políticamente el departamento está dividido en 13 provincias, una de ellas es la provincia de Cajamarca, donde se encuentra la ciudad de Cajamarca, que es la capital del departamento (Banco Central de Reserva del Perú, 2016) (Figura 5-1).

\footnotetext{
${ }^{3}$ Fondo Multilateral de Inversiones, 2014. De las velas a la luz eléctrica. El impacto de la electrificación rural. Disponible en: http://www.fomin.org/Portals/. [Último acceso: Mayo 2016].

${ }^{4}$ United Nations Development Programme, 2009. Human Development Report Peru. Disponible en: http://hdr.undp.org/sites/default/. [Último acceso: Enero 2016].
} 
La provincia de Cajamarca limita al sur con el departamento de la Libertad, al sureste con la provincia de Cajabamba, al norte con la provincia de Hualgayoc, al este con la provincia de San Marcos, al oeste con la provincia de Contumaza, al noreste con Celendín y al noroeste con la provincia de San Pablo (Pontificia Universidad Católica del Perú, 2007) (Figura 5-2).

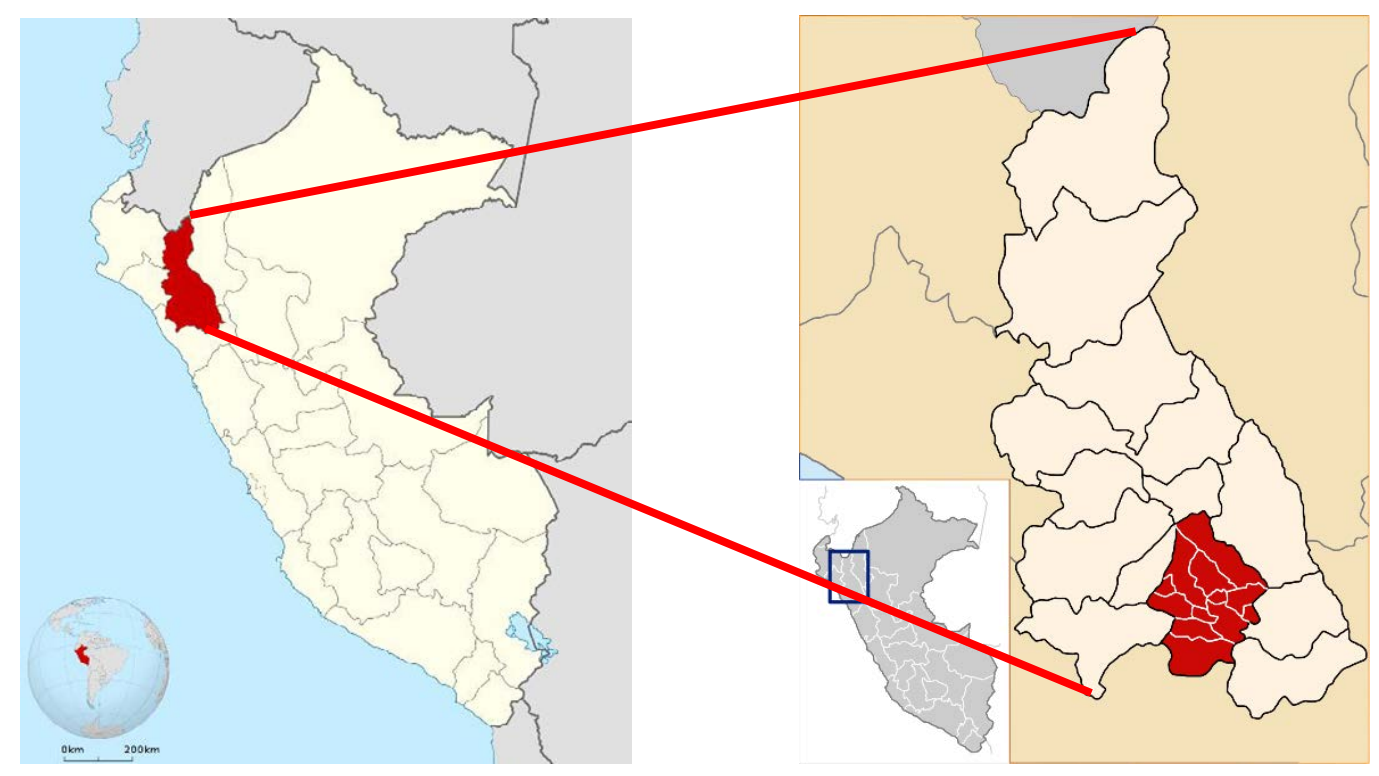

Figura 5-1. Ubicación geográfica de la provincia de Cajamarca.

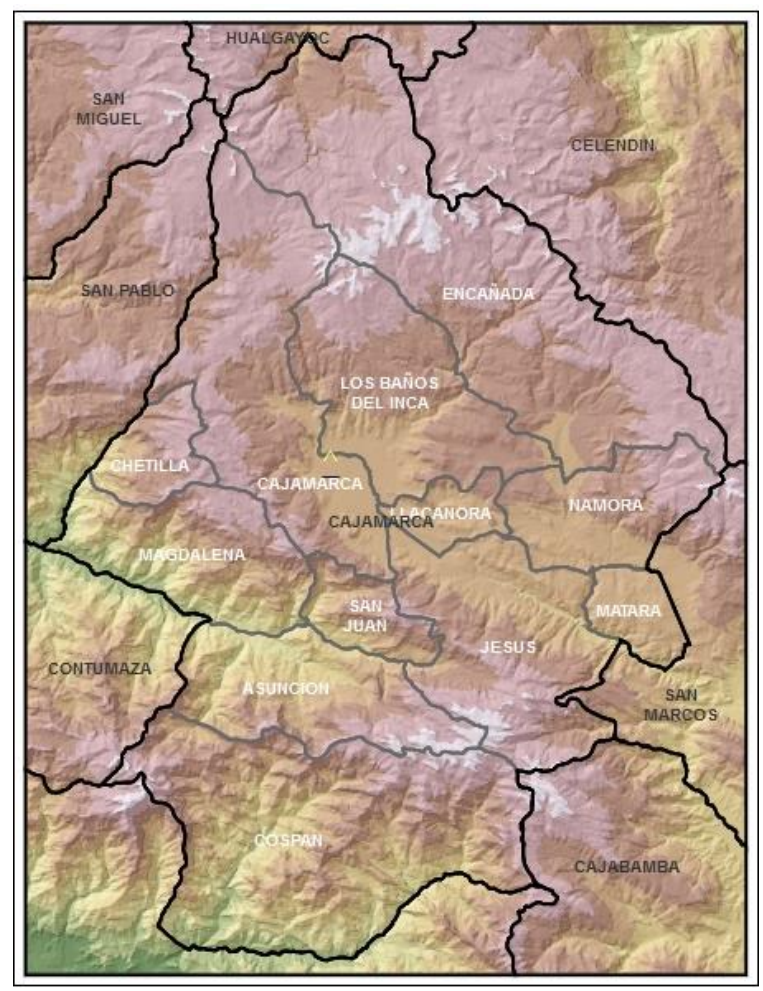

Figura 5-2. Mapa político de la provincia de Cajamarca (Pontificia Universidad Católica del Perú, 2007). 


\subsubsection{Características principales}

Las principales características de la provincia de Cajamarca se recogen en la Tabla 5-1.

Tabla 5-1. Características principales de la provincia de Cajamarca.

\begin{tabular}{|c|c|}
\hline Superficie: & $2.979,78 \mathrm{~km}^{2}(9.02 \% \text { del departamento })^{\mathrm{a}}$ \\
\hline Población: & $389.160(25.37 \% \text { del departamento })^{b}$ \\
\hline Densidad poblacional: & $130,6 \mathrm{hab} / \mathrm{km}^{2}$ \\
\hline Actividades productivas: & $\begin{array}{l}\text { Se destaca la industria minera, con un } 24,5 \% \text { de } \\
\text { participación en el departamento. Seguido de la } \\
\text { agricultura, ganadería, caza y silvicultura }{ }^{c} \text {. }\end{array}$ \\
\hline PBI Per cápita: & 9.843 PEN $~ 3.047$ USD $^{\mathrm{d}}$ \\
\hline Pobreza: & $50,7 \%{ }^{\mathrm{d}}$ \\
\hline Clima: & $\begin{array}{l}\text { Semiseco y templado. La temperatura anual máxima } \\
\text { es de } 22^{\circ} \mathrm{C}\left(71^{\circ} \mathrm{F}\right) \text { y mínima de } 5^{\circ} \mathrm{C}\left(42^{\circ} \mathrm{F}\right)^{\mathrm{b}} \text {. }\end{array}$ \\
\hline $\begin{array}{l}\text { a (Pontificia Universidad C } \\
\text { b (Ministerio de Salud, 20 } \\
\text { c (Banco Central de Reser } \\
\text { d (Instituto Peruano de Ecc }\end{array}$ & $\begin{array}{l}\text { ca del Perú, 2007) } \\
\text { Perú, 2016) } \\
\text { í, 2015) }\end{array}$ \\
\hline
\end{tabular}

\subsection{Estudio de la demanda y oferta energéticas.}

El primer paso para la aplicación del modelo consistió en realizar una revisión del consumo energético en los diferentes sectores productivos de la provincia de Cajamarca. A continuación, se realizó una estimación de la demanda para el horizonte de planificación (Año 2025). Este horizonte es el mismo que se define en el Plan Energético Nacional publicado en 2014 (Ministerio de Energía y Minas, 2014). Para tener en cuenta las políticas energéticas nacionales, se consideraron los mismos supuestos del mencionado plan.

Dentro de esta etapa se realizó también una revisión exhaustiva de información de fuentes oficiales sobre la oferta potencial de energía renovable en el área de estudio.

\subsubsection{Estimación de la demanda energética.}

La demanda de energía en la provincia de Cajamarca para el año 2025 fue estimada en base al Balance Energético Nacional de 2014 (OSINERGMIN, 2015b), donde se indica el consumo de los principales combustibles líquidos de la región, y en base a los consumos históricos de electricidad del INEI (Instituto Nacional de Estadística e 
Informática, 2015), donde se indica el consumo anual de energía eléctrica por departamento. Asimismo, en base a información local, se estimaron los consumos de leñas, carbón vegetal y energía geotérmica.

La estimación de la demanda se realizó proyectando los consumos históricos de electricidad de la región Cajamarca, del 2002 al 2014. A partir de dichos consumos se aplicaron los modelos sumativo y multiplicativo, según el ítem 4.2.1 del Capítulo IV.

Los escenarios de crecimiento del PBI que se asumieron fueron los que figuran en el Plan Energético Nacional 2014-2025 del MINEM, el cual considera valores de crecimiento de 4,5\% y 6,5\%. Las proyecciones se calcularon en base al promedio de los resultados obtenidos de los modelos aplicados (Tabla 5-2).

Tabla 5-2. Proyección de la demanda energética en la región de Cajamarca con los modelos sumativo y multiplicativo (ktep).

\begin{tabular}{|c|c|c|c|c|c|c|c|c|c|}
\hline \multirow[b]{2}{*}{ Año } & \multirow{2}{*}{$\begin{array}{l}\text { Consumo } \\
\text { Electricidad } \\
\text { (ktep) }\end{array}$} & \multicolumn{4}{|c|}{ Escenario 1: $\Delta$ PIB $4.5 \%$} & \multicolumn{4}{|c|}{ Escenario 2: $\Delta$ PIB 6.5\% } \\
\hline & & $\begin{array}{c}\Delta \text { PIB } \\
4.5 \%\end{array}$ & $\mathbf{M S}^{\mathbf{a}}$ & $\mathbf{M M}^{\mathbf{b}}$ & $\begin{array}{c}\text { MF }^{\mathbf{c}} \\
\text { (Promedio) }\end{array}$ & $\begin{array}{l}\Delta \text { PIB } \\
6.5 \%\end{array}$ & MS & MM & $\begin{array}{c}\text { MF } \\
\text { (Promedio) }\end{array}$ \\
\hline 2002 & 4,019 & & & & & & & & \\
\hline 2003 & 4,372 & & & & & & & & \\
\hline 2004 & 5,066 & & & & & & & & \\
\hline 2005 & 5,419 & & & & & & & & \\
\hline 2006 & 7,428 & & & & & & & & \\
\hline 2007 & 8,707 & & & & & & & & \\
\hline 2008 & 9,708 & & & & & & & & \\
\hline 2009 & 10,462 & & & & & & & & \\
\hline 2010 & 11,724 & & & & & & & & \\
\hline 2011 & 13,592 & & & & & & & & \\
\hline 2012 & 14,971 & & & & & & & & \\
\hline 2013 & 16,254 & & & & & & & & \\
\hline 2014 & 18,378 & & & & & & & & \\
\hline 2015 & & 0,045 & 18,382 & 19,205 & 18,794 & 0,065 & 18,384 & 19,573 & 18,978 \\
\hline 2016 & & 0,045 & 18,386 & 20,069 & 19,228 & 0,065 & 18,389 & 20,845 & 19,617 \\
\hline 2017 & & 0,045 & 18,390 & 20,973 & 19,681 & 0,065 & 18,395 & 22,200 & 20,297 \\
\hline 2018 & & 0,045 & 18,394 & 21,916 & 20,155 & 0,065 & 18,401 & 23,643 & 21,022 \\
\hline 2019 & & 0,045 & 18,398 & 22,903 & 20,650 & 0,065 & 18,406 & 25,180 & 21,793 \\
\hline 2020 & & 0,045 & 18,401 & 23,933 & 21,167 & 0,065 & 18,412 & 26,816 & 22,614 \\
\hline 2021 & & 0,045 & 18,405 & 25,010 & 21,708 & 0,065 & 18,417 & 28,559 & 23,488 \\
\hline 2022 & & 0,045 & 18,409 & 26,136 & 22,272 & 0,065 & 18,423 & 30,416 & 24,419 \\
\hline 2023 & & 0,045 & 18,413 & 27,312 & 22,862 & 0,065 & 18,428 & 32,393 & 25,411 \\
\hline 2024 & & 0,045 & 18,417 & 28,541 & 23,479 & 0,065 & 18,434 & 34,498 & 26,466 \\
\hline 2025 & & 0,045 & 18,421 & 29,825 & 24,123 & 0,065 & 18,440 & 36,741 & 27,590 \\
\hline
\end{tabular}

${ }^{\mathrm{a}}$ Modelo Sumativo, ${ }^{\mathrm{b}}$ Modelo Multiplicativo, ${ }^{\mathrm{c}}$ Modelo Final. 
Con los resultados se generó una ecuación de regresión para cada escenario de PBI, obteniendo, para un incremento del 6,5\%, el $\mathrm{R}^{2}$ más favorable (Figura 5-3). Según este resultado se obtuvo una demanda eléctrica para el 2025 de 27,59 ktep, asimismo, se realizó la proyección de los consumos para las distintas fuentes energéticas.

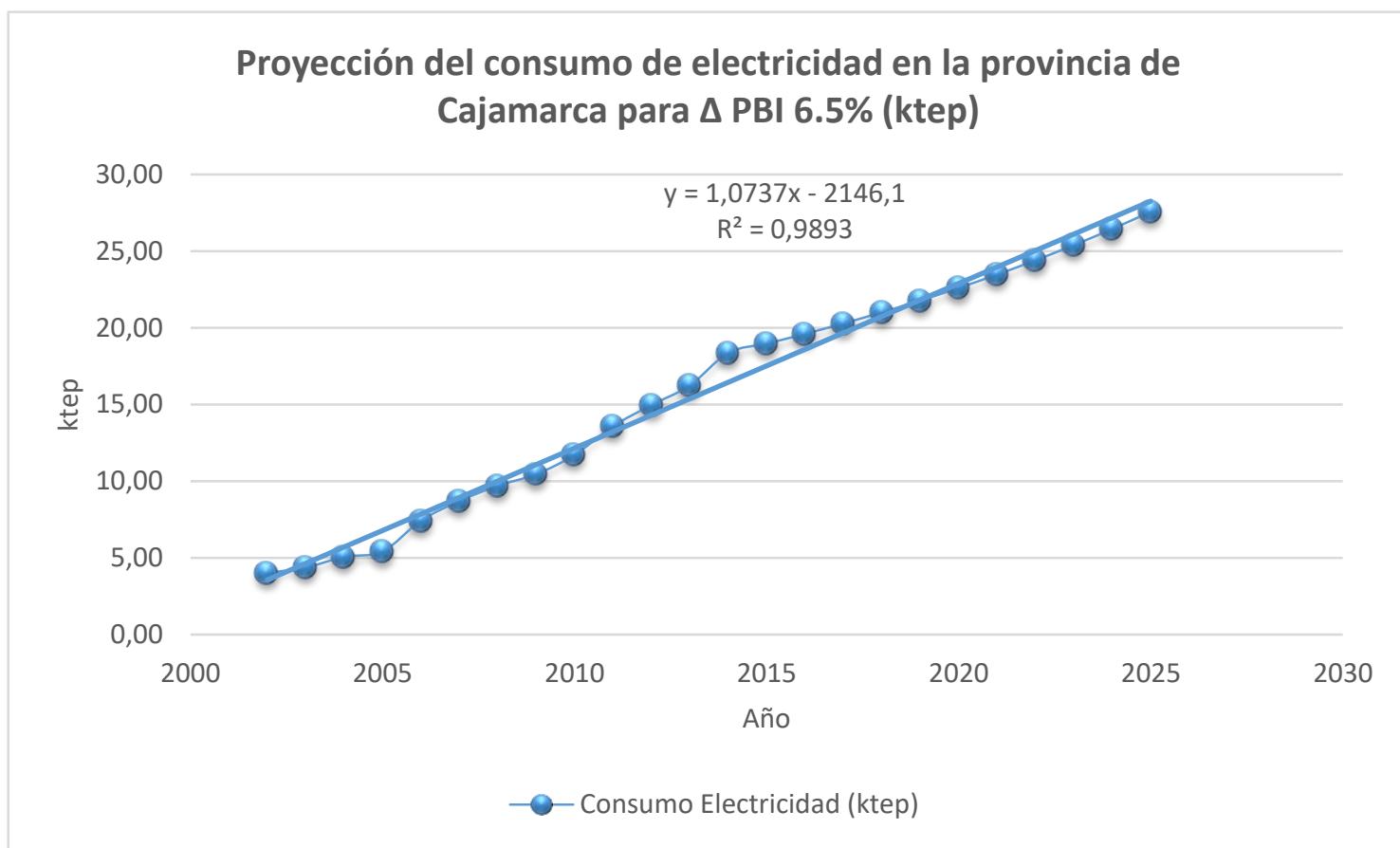

Figura 5-3. Proyección del consumo eléctrico del 2015 al 2025 en la provincia de Cajamarca, para un incremento del PBI del $6.5 \%$.

El consumo de combustibles líquidos en la provincia de Cajamarca fue obtenido del Balance Nacional de Energía de 2014. Dicho consumo en ktep y su proyección al año 2025 se presenta en la Tabla 5-3.

Tabla 5-3. Consumo de combustibles líquidos en la provincia de Cajamarca y su proyección al año 2025.

\begin{tabular}{ccccc}
\hline \multirow{2}{*}{ Combustible } & \multicolumn{2}{c}{ Año 2014 } & \multicolumn{2}{c}{ Año 2025 } \\
\cline { 2 - 5 } & $\begin{array}{c}\text { Transporte } \\
\text { (ktep) }\end{array}$ & $\begin{array}{c}\text { Industrial } \\
\text { (ktep) }\end{array}$ & $\begin{array}{c}\text { Transporte } \\
(\text { ktep })\end{array}$ & $\begin{array}{c}\text { Industrial } \\
\text { (ktep) }\end{array}$ \\
\hline Diésel B5 $^{\text {a }}$ & 10,03 & 17,79 & 14,58 & 25,86 \\
Gasohol $^{\mathrm{b}}$ & 4,20 & 0,06 & 6,11 & 0,09 \\
DB5 S-50 & 0,003 & - & 0,004 & - \\
\hline
\end{tabular}

${ }^{\text {a }}$ Biodiesel al $5 \%$.

${ }^{\mathrm{b}}$ Gasohol al 7,5\%.

${ }^{\mathrm{c}}$ Diésel B5 con un contenido máximo de 50 ppm de azufre.

Según la Tabla 5-3, para el año 2025 se prevé una demanda de combustibles líquidos de 46,64 ktep. 
En la Tabla 5-4 se presenta la demanda de las distintas fuentes energéticas para el año 2025 en la provincia de Cajamarca, por sectores productivos. Asimismo, se ha incluido la estimación de los consumos de leñas, carbón vegetal y energía geotérmica.

Tabla 5-4. Demanda de las principales fuentes energéticas en la provincia de Cajamarca para el año 2025 en ktep.

\begin{tabular}{lccccccc}
\hline Sector & Electricidad & Diésel B5 & $\begin{array}{c}\text { Gasohol } \\
\mathbf{( 7 , 8 \% )}\end{array}$ & DB5 S-50 & Leñas & $\begin{array}{c}\text { Carbón } \\
\text { Vegetal }\end{array}$ & Geotérmica \\
\hline Transporte & & 14,58 & 6,11 & 0,004 & & & \\
$\begin{array}{l}\text { Comercial } \\
\text { Industrial }\end{array}$ & 2,94 & & & & & & \\
$\begin{array}{l}\text { Doméstico } \\
\begin{array}{l}\text { Alumbrado } \\
\text { público }\end{array}\end{array}$ & 9,83 & 25,86 & 0,09 & & 12,69 & 2,70 & 0,205 \\
\hline
\end{tabular}

Según lo mencionado en el apartado 4.5 del Capítulo IV, la demanda energética de una región no puede ser cubierta solamente con energías renovables, sino que sólo podrían cubrir parte de la misma, debido a problemas técnicos, de mercado, de capacidad de instalación etc. En este sentido, en el presente estudio, se han propuesto porcentajes de sustitución acordes con las políticas energéticas nacionales.

El marco regulatorio peruano contempla que, cada 5 años, la generación eléctrica con energías renovables sea el 5\% del consumo eléctrico nacional (Ministerio de Energía y Minas, 2010a). Según esto y teniendo en cuenta el potencial de recursos renovables en la zona, en la presente tesis doctoral se ha establecido un porcentaje de sustitución del $15 \%$ en este sector para el año 2025. Cabe indicar que los 5\% mencionados no incluyen la gran hidráulica, considerada como fuente renovable convencional.

Con respecto a los combustibles para el transporte, en Perú desde el año 2011 se comercializa Diésel B5 y Gasohol (7.8\%) (FAO, 2011). Según la disponibilidad de producción de BL en la provincia de Cajamarca, se estima una sustitución del consumo local en 5\% por el uso de BL al 100\% (biodiesel y etanol).

Dentro del sector industrial, la minera Yanacocha es el único gran consumidor de Diésel B5 y lo utiliza para la generación de energía eléctrica, asimismo, es el principal consumidor de esta fuente energética en la provincia. Debido a esta situación, el presente estudio ha considerado la sustitución del 10\% del consumo de Diésel B5 de dicha minera para introducir el uso de energía solar fotovoltaica. 
Según lo mencionado, en la Tabla 5-5 se presenta la cantidad de energía que se puede sustituir por energías renovables para el horizonte de planificación en ktep.

Tabla 5-5. Energía sustituible por fuentes energéticas renovables para el año 2025 en ktep.

\begin{tabular}{lccc}
\hline \multicolumn{1}{c}{ Sector } & Electricidad & Diésel B5 & Gasohol (7,8\%) \\
\hline Transporte & & 0,73 & 0,31 \\
Comercial & 0,44 & & \\
Industrial & 2,04 & 2,59 & \\
Doméstico & 1,47 & & \\
Alumbrado público & 0,19 & & \\
\hline
\end{tabular}

\subsubsection{Estimación de la oferta energética}

Para el cálculo de la oferta energética de energías renovables se ha realizado un estudio para identificar las fuentes renovables con mayor potencial de aprovechamiento en la zona de estudio. Dichas fuentes renovables fueron descritas en la sección 4.3 del Capítulo IV.

Luego se procedió a la estimación de la oferta de las energías renovables elegidas, para ello se utilizaron fuentes de información nacionales e internacionales (Tabla 5-6).

Tabla 5-6. Fuentes de información utilizadas para la estimación de la oferta de energías renovables en la provincia de Cajamarca.

\begin{tabular}{|c|c|c|c|}
\hline $\mathbf{N}^{\mathbf{0}}$ & Documento & Año & Fuente \\
\hline 1 & Anuario Perú Forestal 2011. & 2011 & $\begin{array}{l}\text { Ministerio de Agricultura } \\
\text { (MINAG) }\end{array}$ \\
\hline 2 & $\begin{array}{l}\text { Bioenergía y seguridad alimentaria "BEFS". } \\
\text { El análisis de BEFS para el Perú. }\end{array}$ & 2010 & FAO \\
\hline 3 & $\begin{array}{l}\text { Elaboración de un índice de Sostenibilidad de } \\
\text { las Cocinas Mejoradas en los departamentos } \\
\text { de Tacna, Moquegua, Arequipa, Ayacucho, } \\
\text { Huancavelica, Cajamarca y San Martin. }\end{array}$ & 2013 & EnDev/GIZ \\
\hline 4 & $\begin{array}{l}\text { Cobertura vegetal y uso actual de la tierra del } \\
\text { departamento de Cajamarca. }\end{array}$ & 2010 & Gobierno Regional de Cajamarca \\
\hline 5 & Atlas de Energía Solar del Perú. & 2003 & $\begin{array}{l}\text { Ministerio de Energía y Minas } \\
\text { (MINEM) }\end{array}$ \\
\hline
\end{tabular}




\begin{tabular}{|c|c|c|c|}
\hline $\mathbf{N}^{\mathbf{0}}$ & Documento & Año & Fuente \\
\hline 6 & $\begin{array}{l}\text { Perfil del proyecto de electrificación de las } \\
\text { localidades de la zona rural de Cachachi, La } \\
\text { Encañada, Namora, San Pablo, Tumbaden e } \\
\text { Ichocan, en la región Cajamarca, empleando } \\
\text { energía solar fotovoltaica }\end{array}$ & 2011 & ACCIONA Microenergía Perú \\
\hline 7 & $\begin{array}{l}\text { Bases definitivas para la subasta de suministro } \\
\text { de electricidad con recursos energéticos } \\
\text { renovables en áreas no conectadas a red. }\end{array}$ & 2014 & OSINERGMIN \\
\hline 8 & $\begin{array}{l}\text { Perú: Estimaciones y proyecciones de } \\
\text { población departamental por años calendario } \\
\text { y edades simples } 1995-2025 \text {. }\end{array}$ & 2010 & $\begin{array}{l}\text { Instituto Nacional de Estadística e } \\
\text { Informática (INEI) }\end{array}$ \\
\hline 9 & Estadística Eléctrica por Regiones. & 2010 & $\begin{array}{l}\text { Ministerio de Energía y Minas } \\
\text { (MINEM) }\end{array}$ \\
\hline 10 & $\begin{array}{l}\text { Energía Solar Térmica. Manual Técnico para } \\
\text { Termas Solares. }\end{array}$ & 2010 & $\begin{array}{l}\text { Green Energy Consultoría y } \\
\text { Servicios SRL }\end{array}$ \\
\hline 11 & $\begin{array}{l}\text { Trabajo Fin de Carrera: "Diseño de una planta } \\
\text { industrial de biodiesel a partir de la semilla de } \\
\text { higuerilla en el valle de Condebamba - } \\
\text { Cajamarca". }\end{array}$ & 2013 & $\begin{array}{l}\text { Universidad Privada del Norte } \\
\text { (Cajamarca) }\end{array}$ \\
\hline 12 & Atlas del Potencial Hidroeléctrico del Perú. & 2011 & $\begin{array}{l}\text { Ministerio de Energía y Minas } \\
\text { (MINEM) }\end{array}$ \\
\hline 13 & $\begin{array}{l}\text { Perú Evaluación del Estado de Preparación de } \\
\text { las Energías Renovables. }\end{array}$ & 2014 & IRENA \\
\hline 14 & $\begin{array}{l}\text { Estudio Hidrológico de la Región Cajamarca } \\
\text { 2010-2011. }\end{array}$ & 2011 & Gobierno Regional de Cajamarca \\
\hline 15 & Atlas Eólico del Perú. & 2008 & $\begin{array}{l}\text { Ministerio de Energía y Minas } \\
\text { (MINEM) }\end{array}$ \\
\hline 16 & I Foro Regional de Energía de Cajamarca. & 2014 & Hidrandina S.A. \\
\hline 17 & $\begin{array}{l}\text { Sistemas Eólicos Pequeños para Generación } \\
\text { de Electricidad. }\end{array}$ & 2002 & $\begin{array}{l}\text { Departamento de Energía de los } \\
\text { Estados Unidos. }\end{array}$ \\
\hline 18 & $\begin{array}{l}\text { Microaerogeneradores de } 100 \text { y } 500 \text { W. } \\
\text { Modelos IT-PE-100 y SP- } 500 .\end{array}$ & 2008 & Soluciones Prácticas ITDG \\
\hline 19 & $\begin{array}{l}\text { Plan Maestro para el Desarrollo de la Energía } \\
\text { Geotérmica en el Perú. }\end{array}$ & 2012 & $\begin{array}{l}\text { Agencia de Cooperación } \\
\text { Internacional del Japón (JICA). }\end{array}$ \\
\hline
\end{tabular}




\subsubsection{Solar Fotovoltaica}

La energía renovable con mayor proyección a ser aprovechada es la solar fotovoltaica. Un ámbito donde existen avances para su aprovechamiento en Perú es en la provisión de electricidad a las zonas rurales. Según datos del 2011, el 16\% población peruana no tiene electricidad en sus casas, cifra que se eleva a $22 \%$ en las zonas rurales (Grupo de Apoyo al Sector Rural de la Pontificia Universidad Católica del Perú, 2017).

En el 2003, en el marco del Proyecto PER/98/G31, el Servicio Nacional de Meteorología e Hidrología (SENAMHI) de Perú elaboró el "Atlas de energía solar del Perú", con el fin de impulsar el uso masivo de la energía solar como fuente energética, especialmente para promover el desarrollo en zonas rurales. La elaboración de este documento permitió cuantificar la disponibilidad de dicha fuente energética, así como conocer su distribución temporal en el territorio peruano (Servicio Nacional de Meteorología e Hidrología, 2003). Según esto, la región de Cajamarca se ubica en una zona con alta disponibilidad de energía solar diaria, entre 5,5 a 6,0 kWh/m² (Figura 5-4).

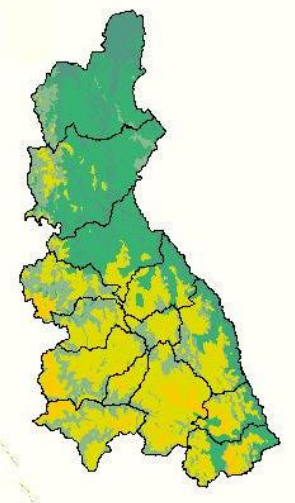

FEBRERO

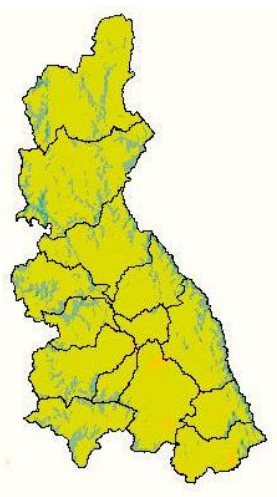

MAYO

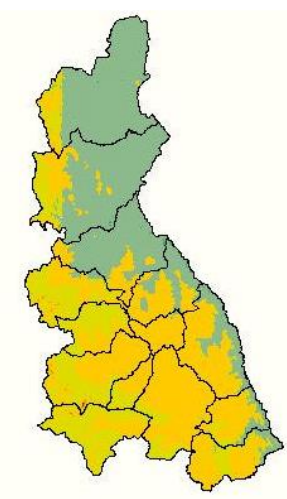

AGOSTO

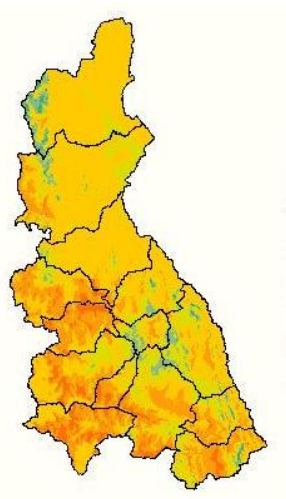

NOVIEMBRE

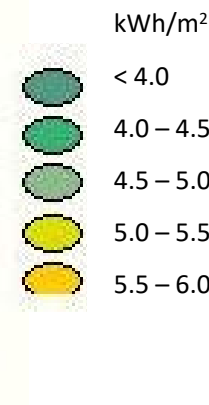

$<4.0$

$0-4.5$

$4.5-5.0$

$5.0-5.5$

$5-6.0$

Figura 5-4. Disponibilidad de energía solar diaria en el departamento de Cajamarca para los meses de febrero, mayo, agosto y noviembre en $\mathrm{kWh} / \mathrm{m}^{2}$ (Servicio Nacional de Meteorología e Hidrología, 2003).

Según la Dirección General de Electrificación Rural aún existen cerca de 500.000 hogares ubicados en zonas rurales que quedarían sin ser atendidos por los programas públicos de electrificación. El Plan Nacional de Electrificación Rural considera que cerca de 345.823 hogares deberán ser cubiertos con módulos fotovoltaicos en espacios rurales (Grupo de Apoyo al Sector Rural de la Pontificia Universidad Católica del Perú, 2017).

Consciente del papel de los paneles fotovoltaicos como la solución a la electrificación rural aislada, el gobierno peruano, en 2013, inició un proceso de subasta 
pública para la instalación de hasta 500.000 sistemas solares en zonas rurales (OSINERGMIN, 2014a). En 2015 la empresa ganadora, Ergon Perú S.A., empezó la ejecución del proyecto con la instalación de 150.000 sistemas a nivel nacional.

Se estima que en la provincia de Cajamarca existen 13.000 familias sin acceso a la energía eléctrica (OSINERGMIN, 2014a).

Actualmente el mayor suministrador de energía solar fotovoltaica en la región es la empresa social ACCIONA Microenergía Perú, con 4.000 familias atendidas en las provincias de Cajamarca, San Pablo, San Marcos y Celendín. Por su parte, la ONG Soluciones Prácticas también ha realizado un importante impulso a la introducción de energías limpias en la región. Este organismo de cooperación técnica internacional, en el año 2009 implementó el proyecto "Acceso a la energía en comunidades de la región Cajamarca a través de la planificación energética participativa con energí s renovables", ejecutado en las provincias de San Pablo y San Marcos, región Cajamarca, con la finalidad de mejorar el acceso a la energía para las CRA de las provincias indicadas, a través de la planificación energética participativa, aprovechando los recursos locales de la zona (Soluciones Prácticas, 2012).

Por otro lado, una oportunidad importante para la introducción de energías renovables en sustitución de combustibles fósiles, viene dada por el consumo a gran escala de Diésel B5 por la minera Yanacocha para la generación de energía eléctrica. Para el horizonte de planificación definido se pretende lograr la sustitución del 10\% de dicho consumo por energía solar fotovoltaica.

\subsubsection{Solar Térmica}

Uno de los ámbitos donde más se ha desarrollado el uso de energía solar en Perú, y el más tradicional, es su aplicación como fuente térmica a través de termas de agua en zonas del sur peruano, principalmente Arequipa y Puno, departamentos en los que existe cerca de 30 empresas dedicadas a la fabricación y mantenimiento de estos sistemas (Piriz, 2013). No obstante, aún es amplio el camino a recorrer para masificar el uso de paneles solares tanto para áreas urbanas como rurales destinados al uso térmico, lo cual implicaría menor consumo de la red eléctrica en los hogares (una terma eléctrica es uno de los principales consumidores de energía eléctrica en un hogar). Asimismo, su uso no se limitaría a lo domestico sino también podría incluirse en usos productivos como secadores 
de granos para la agricultura (en la zona sur la producción de granos andinos como kiwicha, quinua, kañihua es alta) así como para como la potabilización de agua en aquellas zonas que lo requieran.

La energía solar para el calentamiento de agua para usos sanitarios, es una de las aplicaciones que mayor aceptación ha generado en todos los sectores de la población, porque el sol es la fuente más barata para aprovisionarse de agua caliente. En Perú, el consumo de agua caliente para uso doméstico (ducha, lavabo, etc.) es muy variado, dependen de las condiciones socioeconómicas del usuario, mientras que en los centros urbanos el consumo varía entre 10 a 70 litros de agua caliente por persona y por día, en las zonas urbano marginales y rurales el consumo es prácticamente nulo (Piriz, 2013).

Para Perú, los sistemas que usan colectores planos para calentar el agua son ideales. Por la intensa radiación solar en gran parte del país, sistemas simples a precios accesibles son suficientes para la gran mayoría de la población. En promedio, se amortizan en menos de cinco años (Grupo de Apoyo al Sector Rural de la Pontificia Universidad Católica del Perú, 2017).

El Estado peruano y la empresa privada están moviendo el mercado para que existan las condiciones necesarias para masificar estos sistemas. Existe hoy en día una demanda creciente de termas solares y la oferta aún no es suficiente, principalmente, de tecnología nacional que pueda competir sanamente con tecnología extranjera. Este es un nicho de mercado en el que aún hay mucho espacio para los fabricantes e instaladores de estos sistemas (Orbegozo \& Arivilca, 2010).

El sistema de terma solar con circulación natural (efecto termosifón) es el más simple y adecuado para ser construido y utilizado en zonas rurales y a un costo mínimo. Por el hecho de trabajar sin una bomba eléctrica, no requiere conexión a la red de alumbrado público, cosa que es muy ventajosa en el Perú, ya que hay lugares que no cuentan con dicha conexión (Orbegozo \& Arivilca, 2010). Este es el sistema que se considera utilizar en las instalaciones domiciliarias de la provincia de Cajamarca.

\subsubsection{Energía eólica}

Después de impulsar la elaboración del Atlas Solar del Perú en el año 2003, el Ministerio de Energía y Minas, utilizando los alcances del "Programa de Mejoramiento de Electrificación Rural mediante la Aplicación de Fondos Concursables - FONER", 
financiado parcialmente por el Banco Mundial y el Fondo Mundial para el Medio Ambiente (Global Environment Facility - GEF), consideró la realización del Atlas Eólico del Perú (Ministerio de Energía y Minas, 2008). Según dicho documento, el departamento de Cajamarca es la tercera región del país con mayor energía eólica aprovechable ( 3.450 MW). En el caso de la provincia de Cajamarca, hay zonas con una velocidad del viento de $7-8 \mathrm{~m} / \mathrm{s}$ (Figura $5-5$ ).
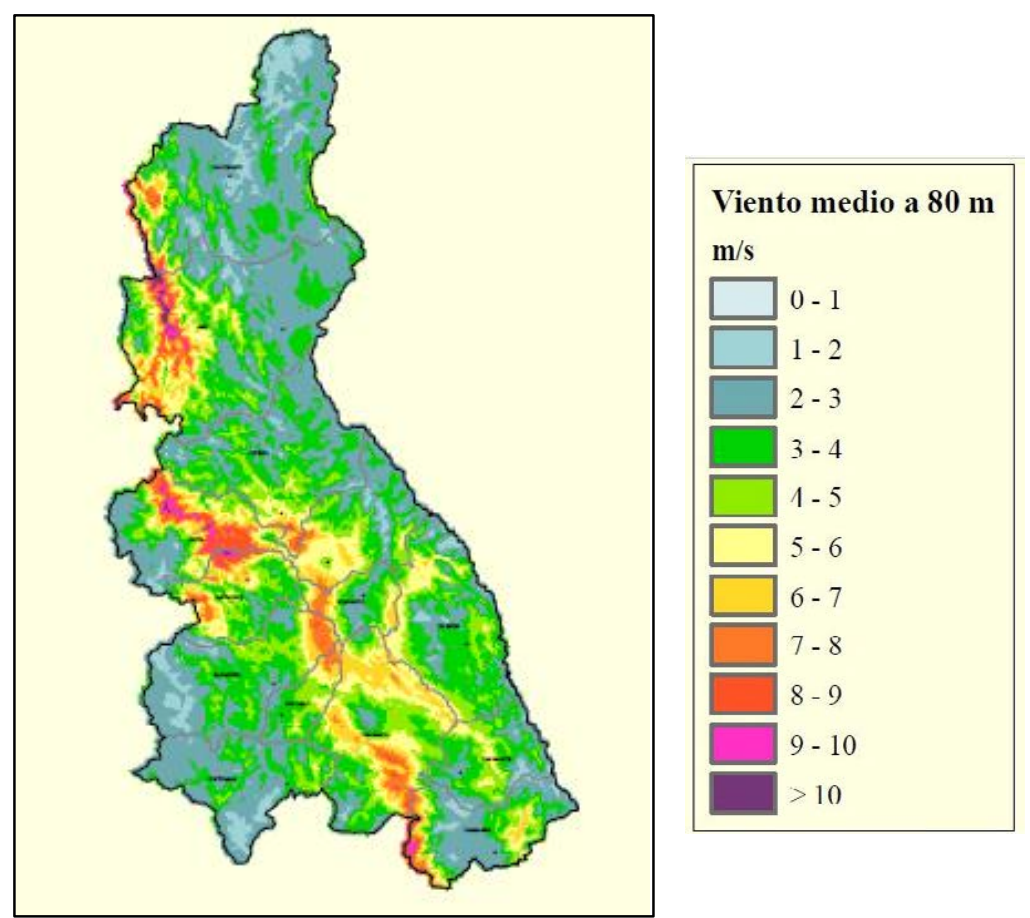

Figura 5-5. Velocidad del viento anual a $80 \mathrm{~m}$ en el departamento de Cajamarca (Ministerio de Energía y Minas, 2008).

Soluciones Prácticas desde el año 2000 trabaja en la investigación y aplicación de la energía eólica de baja potencia para la electrificación del sector rural. Como experiencia de electrificación, ha logrado dotar de electricidad con energía eólica de pequeña escala a las comunidades de El Alumbre, Campo Alegre, Alto Perú, todas ellas ubicadas en la provincia de Cajamarca (Velásquez, 2013). Son principalmente sistemas domiciliarios combinados con energía solar fotovoltaica (sistemas híbridos).

\subsubsection{Mini hidráulica}

La generación de energía eléctrica suministrada a la provincia de Cajamarca por el Sistema Interconectado Centro Norte (SICN), se caracteriza por el predominio de centrales de generación hidráulica (aproximadamente el 75\% de la capacidad instalada es hidroeléctrica) (Indacochea, et al., 1998). 
En los países andinos donde existen caídas de agua naturales, la solución más barata y sostenible es el aprovechamiento de las caídas para generar electricidad, accionando con la energía del agua una turbina o máquina motriz hidráulica que a su vez impulse un generador eléctrico produciendo así la energía eléctrica sin tener que quemar el petróleo ni utilizar costosos y escasos repuestos para los motores (Gobierno Regional de Cajamarca, 2011). Según el Estudio Hidrológico de la Región Cajamarca 2010-2011, realizado por el Gobierno Regional, existen tres ríos importantes en la provincia, que pertenecen a las cuencas hidrográficas Crisnejas y Jequetepeque, los cuales tendrían potencial hidroenergético.

Según esta información se ha estimado el potencial de explotación de la mini hidráulica en la provincia, el cual es del orden de 6.2 MW para generación de energía eléctrica. Por otro lado, se ha tenido en cuenta la potencia instalada de las 40 micro centrales hidroeléctricas que Soluciones Prácticas ha instalado en la región (Soluciones Prácticas ITDG, 2015).

\subsubsection{Biomasa}

En esta alternativa energética se consideran la leña y el carbón vegetal. En el marco del proyecto Bioenergía y Seguridad Alimentaria realizado por la FAO en el 2010, se estimó que el consumo de leña y carbón total del país fue cerca de 4 millones de toneladas por año, aunque informes oficiales estiman que llega a los 5 millones de toneladas anuales; estas discrepancias se debieron principalmente a la falta de información, lo cual no permitió que algunas ciudades fueran evaluadas correctamente en el análisis. En base a los resultados obtenidos, las provincias que presentan el mayor consumo de biomasa son: Virú (La Libertad) Chota (Cajamarca), Morropón (Piura) y Tarma (Junín). En la sierra de Perú se han plantado más de 725.000 ha principalmente de Eucalyptus sp. y de Pinus sp., particularmente en las regiones administrativas de Cuzco, Cajamarca, Ancash, Junín, Apurímac y Ayacucho (FAO, 2011).

El departamento de Cajamarca es el mayor productor de leña del Perú, representado el $14 \%$ de la producción nacional y, por lo tanto, la región donde más se consume este energético (Ministerio de Agricultura, 2012). En la provincia de Cajamarca se ubica la Granja Porcón donde se encuentra el bosque de Pinos más grande del Perú con 12.800 ha de extensión; estas plantaciones son manejadas por la Asociación Civil para la Investigación y Desarrollo Forestal (ADEFOR) (Carton, 1996). La producción de leña 
del bosque y de los setos vivos de las terrazas produjo un cambio considerable en el bienestar de las familias e interrumpió definitivamente la depredación de los montes (Carton, 1996).

El uso de leña en la provincia se extiende no sólo al ámbito rural sino también al urbano, donde muchos hogares han implementado hornos de barro y cocinas mejoradas como complemento a la cocina a gas. En este sentido, el programa de acceso a la energía EnDev GIZ ha instalado, con el apoyo de instituciones públicas y privadas, 18.545 cocinas mejoradas en la provincia de Cajamarca (Díaz, et al., 2013). Eliminar el uso de fogones de tres piedras se ha convertido en una prioridad absoluta en los programas de acceso universal a la energía en entornos rurales. La razón radica en los graves daños que causan los contaminantes intradomiciliarios en la salud de las personas y especialmente en los más expuestos, que son las mujeres y los niños. Con el uso de cocinas mejoradas se consigue un $50 \%$ de eficiencia en el consumo de leña, lo que produciría una reducción de 900.000 toneladas de leña al año en el país (Consorcio de Investigación Económica y Social, 2016).

Con respecto al carbón vegetal, según el estudio de la FAO antes mencionado, la región natural que produce mayor cantidad de leña es la Costa con casi la totalidad de producción de carbón del país $(97,35 \%)$. En esta región se extrae madera para leña y para producir carbón vegetal, principalmente de los algarrobos, para uso doméstico. De manera ilegal se extrae esta especie para producir carbón vegetal destinado a restaurantes y pollerías, así como para utilizarlo en parrilladas (FAO, 2010).

En la provincia de Cajamarca el consumo de carbón de vegetal es aún reducido y muy inferior al de la leña, sin embargo, se ha considerado como opción energética en el presente estudio ya que representa un potencial promisorio para el aumento de la eficiencia durante la cocción de alimentos, al poseer un poder calorífico $50 \%$ superior al de la leña.

\subsubsection{Biocombustibles Líquidos}

Existe un potencial importante para el uso de ricino (Ricinus communis L.) con el fin de producir biodiesel en el Valle de Condebamba. Se ha estimado una plantación de 2.000 hectáreas y una producción de 1.306 litros de petróleo por hectárea (Campos \& Salazar, 2013). 
Existe un gran interés en la selva de Perú para la promoción de cultivos de caña de azúcar para producción de etanol hidratado para uso combustible principalmente en motocicletas. La actual producción de caña de azúcar en la selva es relativamente pequeña comparada con los niveles de producción de la costa peruana y está principalmente destinada al consumo local de azúcares (FAO, 2010). La zona norte de Cajamarca (San Ignacio y Jaén), que pertenece a la selva peruana, podría convertirse en lugar para la potencial explotación de caña de azúcar y producción de etanol. Cabe indicar que existe unanimidad de criterios en el tema de utilizar las tierras deforestadas, para la producción de cultivos energéticos (SNV, 2007).

\subsubsection{Geotérmica}

La energía geotérmica es otra fuente energética con un importante potencial para ser aprovechado en la provincia de Cajamarca. En el año 2012 el Ministerio de Energía y Minas de Perú con el apoyo de la Agencia de Cooperación Internacional del Japón (JICA), elaboró el Plan Maestro para el Desarrollo de la Energía Geotérmica en el Perú. De acuerdo a este documento, se estima que existe un potencial considerable de recursos geotérmicos aptos para la generación eléctrica en el Perú, que bien podrían superar los 3.000 MW. Sin embargo, en la actualidad no hay ningún emprendimiento de explotación de este recurso dado a que el país no ha establecido los conocimientos técnicos y la experiencia necesaria para la exploración, desarrollo y explotación de los recursos geotérmicos. Por otro lado, el estudio pone de manifiesto el potencial energético existente en la provincia de Cajamarca, específicamente en el distrito de Baños del Inca, donde se estima una producción de 29 MW para generación de energía eléctrica (Agencia de Cooperación Internacional del Japón, 2012).

Sin embargo, se prevé que la introducción de la tecnología necesaria para la generación de energía eléctrica a partir del recurso geotérmico sea a largo plazo, posterior al año 2025. Por ello, en la presente tesis doctoral, se considera el uso directo de dicho recurso como fuente de calor en viviendas y escuelas del distrito Baños del Inca, ubicado a 20 minutos de la ciudad de Cajamarca.

\subsection{Establecimiento de las restricciones}

Teniendo en cuenta la descripción y análisis realizados en el apartado 5.2 fueron calculadas las restricciones del modelo. 
Los valores obtenidos para este caso de estudio fueron clasificados de acuerdo al apartado 4.5 del Capítulo IV y se muestran a continuación. Tal como se indicó en dicho aparatado, el sector eléctrico se analizó por separado debido a sus características especiales.

Restricciones basadas en la demanda de energía y el potencial RE real (en ktep):

$\mathrm{ST} \leq 1,66^{\mathrm{a}}$
$\mathrm{LEN} \leq 12,70^{\mathrm{b}}$
$\mathrm{CV} \leq 1,70^{\mathrm{b}}$
$\mathrm{BL} \leq 03^{\mathrm{c}}$
$\mathrm{GEO} \leq 1,20^{\mathrm{d}}$

Electricidad:

$\mathrm{PV}+\mathrm{EOL}+\mathrm{MH} \leq 6.72$

$\mathrm{PV} \leq 3,36^{\mathrm{e}}$

$\mathrm{EOL} \leq 2,39^{\mathrm{f}}$

$\mathrm{MH} \leq 3,72^{\mathrm{g}}$

${ }^{\text {a }}$ Calculado en base a INEI (2010) y Orbegozo et al. (2010)

${ }^{\mathrm{b}}$ Calculado en base a INEI (2008), Diaz et al. (2013) y FAO (2010)

${ }^{c}$ Calculado en base a OSINERGMIN (2015), Campos et al. (2013) y Pérez et al. (2015)

${ }^{\mathrm{d}}$ Calculado en base a INEI (2010) y JICA (2012)

e Calculado en base a SENAMHI (2003), AMP (2011), OSINERGMIN (2014), INEI (2010) y Hidrandina S.A. (2014)

${ }^{\mathrm{f}}$ Calculado en base a MINEM (2008), Soluciones Prácticas - ITDG (2008) y Departamento de Energía, EE.UU. (2002)

g Calculado en base a IRENA (2014), López (2014), Gobierno Regional de Cajamarca (2011) y

Soluciones Prácticas - ITDG (2010)

Restricciones basadas en energías renovables ya existentes (en ktep):

$\mathrm{ST} \geq 0,10^{\mathrm{a}}$
$\mathrm{LEN} \geq 1,70^{\mathrm{a}}$
$\mathrm{CV} \geq 1,00^{\mathrm{a}}$
$\mathrm{GEO} \geq 0,05^{\mathrm{a}}$
$\mathrm{BL} \geq 0,74^{\mathrm{b}}$
$\mathrm{PV} \geq 2,64^{\mathrm{c}}$
$\mathrm{EOL} \geq 0,01^{\mathrm{d}}$
$\mathrm{MH} \geq 0,50^{\mathrm{d}}$

${ }^{\text {a }}$ Estimado por el autor.

${ }^{\mathrm{b}}$ Cantidad mínima para la sustitución de Diésel B5 en el transporte. Actualmente no existe producción de BL.

${ }^{\mathrm{c}}$ Cantidad mínima para la sustitución de Diésel B5 utilizado para generar energía eléctrica por la minera Yanacocha. Se consideró una potencia instalada de 0,06 ktep para PV en la provincia de Cajamarca.

${ }^{\mathrm{d}}$ Estimado en base a Soluciones Prácticas - ITDG (2015). 


\subsection{Funciones Objetivo}

\subsubsection{F1: Maximización de la sustitución de las energías fósiles por energías renovables.}

En Perú, la política energética se orienta decididamente hacia el uso progresivo de las energías renovables. En el ámbito eléctrico, más allá de las centrales de generación renovables convencionales (hidroeléctricas) que vienen operando en el país hace muchos años, se continuará con la promoción de las energías renovables no convencionales, entre ellos la energía eólica, solar, mini hidráulica, etc. (Ministerio de Energía y Minas, 2014).

Dicha coyuntura se puede trasladar también a la provincia de Cajamarca, donde las condiciones climáticas y orográficas favorecen el uso de las energías renovables, pero que, por la falta de planificación, su aprovechamiento es deficiente. La minería, por ejemplo, que representa la actividad económica más importante de la región, utiliza, principalmente, combustibles fósiles para generar energía eléctrica. De aquí deriva la necesidad de incorporar una función objetivo que maximice la oferta de energías renovables.

Según lo mencionado en el capítulo anterior, los coeficientes de la función objetivo $\mathrm{F}_{1}$ se obtuvieron a través de la consulta a expertos, cuyo perfil fue el siguiente:

- Experiencia profesional en el campo de la planificación y política energética, en el sector público y privado de Perú.

- Experiencia práctica en la aplicación de tecnologías energéticas renovables en zonas urbanas y rurales de la provincia de Cajamarca, y conocimiento de su repercusión social.

- Experiencia en la creación de alianzas estratégicas con entidades públicas locales y en el trabajo comunitario con pobladores de las comunidades rurales aisladas de la provincia de Cajamarca.

- Conocimiento de la regulación tarifaria energética para las energías renovables y oportunidades de negocio de este rubro en la provincia de Cajamarca y en el mercado nacional.

- Experiencia en la cooperación para el desarrollo en el campo del acceso a la energía para comunidades rurales aisladas. Visión global del estado de la electrificación rural fuera de la red en Latinoamérica. 
Mediante dicha consulta se obtuvo la valoración de las alternativas energéticas consideradas, según los niveles de comparación establecidos en la Tabla 4-2. Los resultados se muestran en la Tabla 5-7.

Tabla 5-7. Evaluación de niveles de comparación para $F_{1}$.

\begin{tabular}{lcccccccc}
\hline & PV & ST & EOL & LEN & CV & BL & MH & GEO \\
\hline Demanda Tecnológica & 4 & 4 & 4 & 2 & 2 & 2 & 5 & 3 \\
Capacidad de investigación & 3 & 3 & 3 & 4 & 4 & 3 & 4 & 2 \\
Ampliación de la experiencia & 5 & 4 & 3 & 3 & 5 & 2 & 4 & 4 \\
Mejora de la tecnología & 4 & 3 & 3 & 3 & 3 & 3 & 3 & 3 \\
Nivel tecnológico adquirido & 4 & 2 & 3 & 2 & 3 & 2 & 4 & 2 \\
Tiempo de operación & 5 & 5 & 4 & 5 & 5 & 2 & 4 & 5 \\
Facilidad de implementación & 4 & 5 & 4 & 5 & 4 & 4 & 3 & 5 \\
Capacidad de integración & 5 & 4 & 5 & 5 & 5 & 5 & 5 & 5 \\
Demanda potencial & 4 & 5 & 4 & 3 & 3 & 3 & 5 & 4 \\
Disponibilidad de oferta & 5 & 5 & 3 & 5 & 4 & 2 & 3 & 4 \\
Interés de política energética & 5 & 4 & 5 & 2 & 2 & 5 & 5 & 4 \\
Precio actual & 2 & 3 & 3 & 2 & 2 & 2 & 5 & 4 \\
Demanda social & 4 & 4 & 4 & 2 & 2 & 2 & 5 & 4 \\
Legislación prevista & 5 & 3 & 4 & 3 & 3 & 5 & 4 & 3 \\
Aspectos ambientales & 4 & 5 & 5 & 2 & 2 & 5 & 2 & 5 \\
\hline
\end{tabular}

Los valores de N y M fueron calculados según (4) y (5). Los resultados obtenidos se muestran en la Tabla 5-8.

Tabla 5-8. Cálculo de factores $\mathrm{M}$ y $\mathrm{N}$ para $\mathrm{F}_{1}$.

\begin{tabular}{cccc}
\hline Alternativa & $\mathbf{M}$ & $\mathbf{N}$ & Coeficiente \\
\hline PV & $1,5^{*} 10^{9}$ & $1,5^{*} 10^{3}$ & 2,5 \\
MH & $8,6^{*} 10^{8}$ & $3,5^{*} 10^{3}$ & 2,5 \\
ST & $5,2^{*} 10^{8}$ & $1,0^{*} 10^{4}$ & 2 \\
EOL & $3,7^{*} 10^{8}$ & $4,7^{*} 10^{4}$ & 2 \\
GEO & $2,8^{*} 10^{8}$ & $2,8^{*} 10^{4}$ & 2 \\
CV & $2,1^{*} 10^{7}$ & $6,6^{*} 10^{5}$ & 1,5 \\
LEN & $1,3^{*} 10^{7}$ & $6,6^{*} 10^{5}$ & 1,5 \\
BL & $8,6^{*} 10^{6}$ & $8,8^{*} 10^{5}$ & 1 \\
\hline
\end{tabular}

Luego de obtener los coeficientes, la ecuación final para $F_{1}$ fue la siguiente: $\mathrm{F}_{1}=2,5 \mathrm{PV}+2,5 \mathrm{MH}+2 \mathrm{ST}+2 \mathrm{EOL}+2 \mathrm{GEO}+1,5 \mathrm{CV}+1,5 \mathrm{LEN}+\mathrm{BL}$

\subsection{2 $\mathrm{F}_{2}, \mathrm{~F}_{3}$ y $\mathrm{F}_{4}$ : Maximización de las emisiones evitadas de $\mathrm{CO}_{2}, \mathrm{NO}_{\mathbf{x}}$ y $\mathrm{SO}_{2}$, respectivamente.}


En Perú, las mayores emisiones de GEI son producidas por la deforestación y el cambio de uso de la tierra, actividades que liberan $\mathrm{CO}_{2}$ a la atmósfera. Aunque las emisiones de GEI generadas en el país representan sólo el 0,4\% de las emisiones mundiales, éstas continúan creciendo. Dichas emisiones están relacionadas con las tendencias demográficas, los patrones de consumo, los cambios económicos y tecnológicos, entre otros (OSINERGMIN, 2014b).

La generación de electricidad a partir de recursos energéticos renovables (RER) en el país ha mitigado la emisión de 2.084 miles de toneladas de $\mathrm{CO}_{2}$ equivalente $\left(\mathrm{tCO}_{2} \mathrm{e}\right)$. Esto ocurrió desde el inicio de las operaciones de la primera central RER hasta 2013 (Ministerio de Energía y Minas, 2010b).

En Cajamarca, las principales emisiones de GEI son producidas por la combustión de combustibles para el transporte y el destinado para centrales térmicas. Además del $\mathrm{CO}_{2}$, estas actividades producen dos contaminantes atmosféricos: $\mathrm{NO}_{\mathrm{X}}$ y $\mathrm{SO}_{2}$. Las emisiones de $\mathrm{NO}_{\mathrm{X}}$ se deben principalmente al mal estado de los vehículos en la ciudad (Consejo Nacional del Ambiente, 2005).

Según lo mencionado y teniendo en cuenta el Inventario Nacional de GEI de Perú en 2000 (Ministerio del Ambiente, 2009), los coeficientes para las funciones de $\mathrm{CO}_{2}, \mathrm{NO}_{\mathrm{X}}$ y $\mathrm{SO}_{2}$ fueron: 7,004 $\mathrm{tCO}_{2}$ / tep, $0,020 \mathrm{tNO}_{\mathrm{X}}$ / tep y $0,029 \mathrm{tSO}_{2}$ / tep, respectivamente.

La Tabla 5-9 muestra un resumen de las emisiones del ciclo de vida correspondientes a los diferentes tipos de energía renovable que se han considerado para calcular los coeficientes de las funciones ambientales.

Tabla 5-9. Emisiones de ciclo de vida de $\mathrm{CO}_{2}$ de ER; $\mathrm{tCO}_{2} / \mathrm{tep}, \mathrm{tNO}_{\mathrm{x}} / \mathrm{tep}, \mathrm{tSO}_{2} / \mathrm{tep}$ (Phen, 2006).

\begin{tabular}{ccccccc}
\hline $\begin{array}{l}\text { Emisiones } \\
\text { de ciclo de } \\
\text { vida }\end{array}$ & PV & ST & EOL & LEN & MH & GEO \\
\hline $\mathrm{CO}_{2}$ & 1,1512 & 0,1558 & 0,1186 & 0,1628 & 0,1512 & 0,4395 \\
$\mathrm{NO}_{\mathbf{X}}$ & 0,004 & 0,0085 & 0,0004 & 0,003 & 0,0006 & 0,0022 \\
$\mathrm{SO}_{2}$ & 0,0033 & 0,0005 & 0,0005 & 0,0003 & 0,0003 & 0,0007 \\
\hline
\end{tabular}

En cuanto a $\mathrm{BL}$, la cantidad de emisiones de $\mathrm{CO}_{2}$ evitadas se ha calculado considerando que el Diésel B5 es principalmente el combustible a sustituir. De acuerdo a esto y teniendo en cuenta que el ciclo de vida de las emisiones de $\mathrm{CO}_{2}$ para $\mathrm{BL}$ es 0,8129 $\mathrm{tCO}_{2} /$ tep (IRENA, 2016), las emisiones de $\mathrm{CO}_{2}$ evitadas se estimaron en 1,8687 $\mathrm{tCO}_{2} /$ tep. Cuando los biocombustibles sustituyen a la gasolina y al diésel en el sector del transporte, 
las emisiones de $\mathrm{SO}_{2}$ se reducen. Sin embargo, en el caso del NOx, la reducción de las emisiones depende del patrón de sustitución y de la tecnología (Domínguez \& Marcos, 2015). Por lo tanto, muchos estudios reportan mayores emisiones de NOx con biodiesel que el diésel de petróleo (Buyukkaya, 2010). Por esta razón, las emisiones evitadas de NOx no han sido consideradas. Las emisiones de $\mathrm{SO}_{2}$ evitadas se obtuvieron del Inventario Nacional de GEI del Perú en el año 2000. Este valor fue de 0,0071 $\mathrm{tSO}_{2} /$ tep.

Cabe destacar que se utilizaron indicadores ambientales nacionales debido a la escasa información en la provincia de Cajamarca. Esta fue una limitación para el cálculo de estos coeficientes.

A continuación, se muestran las funciones correspondientes:

$$
\begin{aligned}
& \mathrm{F}_{2}=6,544 \mathrm{PV}+7,004 \mathrm{ST}+6,884 \mathrm{MH}+2,7 \mathrm{BL}+6,374 \mathrm{CV}+7,004 \mathrm{GEO}+6,884 \mathrm{EOL} \\
& +6,484 \mathrm{LEN}
\end{aligned}
$$

$\mathrm{F}_{3}=0,0181 \mathrm{PV}+0,0198 \mathrm{ST}+0,0197 \mathrm{MH}+0,0115 \mathrm{CV}+0,0198 \mathrm{GEO}+0,0194 \mathrm{EOL}+$ $0,0115 \mathrm{LEN}$

$\mathrm{F}_{4}=0,026 \mathrm{PV}+0,029 \mathrm{ST}+0,029 \mathrm{MH}+0,0058 \mathrm{BL}+0,0286 \mathrm{CV}+0,029 \mathrm{GEO}+0,0285$ $\mathrm{EOL}+0,0286 \mathrm{LEN}$

\subsubsection{F5: Minimización de los costes.}

Los valores de LCOE (USD/tep) para cada alternativa energética se obtuvieron de las siguientes fuentes:

- Agencia Internacional de Energías Renovables. Costes de la generación energética renovable - Informe 2014 (IRENA, 2015).

- Lazard. Levelized Cost of Energy Analysis - Version 10.0 (Lazard, 2016).

- Instituto de Tecnologías Sostenibles de Austria. Solar Heat Worldwide (Mauthner, et al., 2016).

De acuerdo con esto, la ecuación obtenida fue la siguiente:

$\mathrm{F}_{5}=2.093 \mathrm{ST}+3.488 \mathrm{PV}+3.372 \mathrm{EOL}+698 \mathrm{LEN}+698 \mathrm{CV}+1.081 \mathrm{BL}+849 \mathrm{MH}+$ 465 GEO 


\subsubsection{F6: Maximización del uso de alternativas energéticas renovables para el suministro sostenible de energía en CRA.}

En la Tabla 5-10 se muestran los resultados de la evaluación de niveles de comparación realizada por un comité de expertos para cada alternativa energética.

Tabla 5-10. Evaluación de niveles de comparación para $F_{6}$.

\begin{tabular}{|c|c|c|c|c|c|c|c|c|}
\hline & $\mathbf{P V}$ & ST & $\overline{\text { EOL }}$ & LEÑ & $\mathbf{C V}$ & $\overline{B L}$ & MH & GEO \\
\hline Bajo LCOE & 2 & 3 & 3 & 4 & 4 & 3 & 4 & 2 \\
\hline Bajo costo de mantenimiento & 4 & 4 & 4 & 4 & 4 & 2 & 3 & 3 \\
\hline Fácil instalación y uso & 5 & 3 & 4 & 5 & 5 & 3 & 2 & 3 \\
\hline Larga vida útil & 5 & 4 & 4 & 4 & 4 & 4 & 4 & 4 \\
\hline Necesidad de batería & 5 & 5 & 2 & 5 & 5 & 5 & 5 & 5 \\
\hline Corriente Alterna & 5 & 2 & 5 & 5 & 5 & 5 & 5 & 2 \\
\hline Modularidad de equipos & 4 & 5 & 4 & 1 & 1 & 1 & 1 & 3 \\
\hline Capacitación requerida & 3 & 3 & 2 & 5 & 5 & 5 & 3 & 2 \\
\hline Escalabilidad y replicabilidad & 4 & 4 & 2 & 4 & 4 & 2 & 2 & 2 \\
\hline Aceptación y participación social & 4 & 4 & 3 & 4 & 4 & 2 & 5 & 4 \\
\hline Impacto social directo & 4 & 3 & 5 & 1 & 1 & 2 & 3 & 2 \\
\hline Bajo impacto ambiental & 4 & 5 & 5 & 2 & 2 & 2 & 4 & 4 \\
\hline $\begin{array}{l}\text { Generación de puestos de trabajo } \\
\text { permanentes }\end{array}$ & 3 & 4 & 4 & 2 & 2 & 2 & 3 & 2 \\
\hline Disponibilidad de fuente energética & 5 & 4 & 4 & 3 & 3 & 1 & 4 & 2 \\
\hline Fácil hibridación & 3 & 1 & 5 & 2 & 2 & 3 & 1 & 1 \\
\hline Baja generación de residuos & 4 & 4 & 4 & 3 & 3 & 4 & 3 & 4 \\
\hline Legislación tarifaria vigente & 3 & 2 & 4 & 2 & 2 & 4 & 2 & 1 \\
\hline Seguridad de equipos frente a robos & 3 & 3 & 3 & 4 & 4 & 5 & 5 & 4 \\
\hline Necesidad de importar equipos & 4 & 4 & 2 & 5 & 5 & 5 & 4 & 3 \\
\hline
\end{tabular}

Los valores de $\mathrm{M}$ y $\mathrm{N}$ se indican en la Tabla 5-11.

Tabla 5-11. Cálculo de factores $\mathrm{M}$ y $\mathrm{N}$ para $\mathrm{F}_{6}$.

\begin{tabular}{lccc}
\hline Alternativa & $\mathbf{M}$ & $\mathbf{N}$ & Coeficiente \\
\hline PV & $8,4^{*} 10^{10}$ & $8,6^{*} 10^{3}$ & 2,5 \\
EOL & $1,8^{*} 10^{10}$ & $1,8^{*} 10^{6}$ & 2 \\
ST & $8,0^{*} 10^{9}$ & $5,0^{*} 10^{6}$ & 2 \\
LEN & $1,8^{*} 10^{9}$ & $3,7 * 10^{6}$ & 1,5 \\
CV & $1,8^{*} 10^{9}$ & $3,7 * 10^{6}$ & 1,5 \\
MH & $1,2 * 10^{9}$ & $1,2 * 10^{7}$ & 1,5 \\
BL & $3,5^{*} 10^{8}$ & $2,2 * 10^{7}$ & 1 \\
GEO & $5,3^{*} 10^{7}$ & $1,1 * 10^{9}$ & 1 \\
\hline
\end{tabular}

Según lo expuesto, la función objetivo $\mathrm{F}_{6}$ tuvo la siguiente forma:

$\mathrm{F}_{6}=2,5 \mathrm{PV}+2 \mathrm{EOL}+2 \mathrm{ST}+1,5 \mathrm{LEN}+1,5 \mathrm{CV}+1,5 \mathrm{MH}+\mathrm{BL}+\mathrm{GEO}$ 


\subsection{Retroalimentación}

Según se indicó en el Capítulo IV, un paso importante en el proceso de planificación es la retroalimentación del modelo. Este ejercicio es realizado con la función $F_{1}$ (maximización de las energías renovables), permitiendo detectar en los primeros resultados valores imposibles de alcanzar a nivel de vector energético o sectorial y, según eso, aplicar los cambios necesarios en los coeficientes de las funciones objetivo o en las restricciones.

En el presente estudio, durante el proceso de retroalimentación, se encontró que el resultado obtenido para los Biocombustibles Líquidos (BL) estaba por encima del valor estimado como potencial de producción en la zona de estudio. Esto sucedió por pretender sustituir el Diésel B5 y el gasohol con dicha alternativa energética. Tal como se indicado en el Apartado 5.2.1, la mayor parte del Diésel B5 es consumido por las plantas térmicas de la minera local. En vista de ello y por la oportunidad que puede representar para una sustitución importante de energía fósil, se consideró el reemplazo parcial de Diésel B5 utilizado en dichas plantas, por energía solar fotovoltaica (PV). Como medida correctiva se modificaron las restricciones correspondientes a dichos vectores energéticos, quedando los valores tal como se han indicado en el Apartado 5.3. 


\section{CAPÍTULO VI}

\section{RESULTADOS Y DISCUSIÓN}

En este capítulo se muestran los resultados obtenidos con el programa Matlab®. Se calcularon las distancias $\mathrm{L}_{1}, \mathrm{~L}_{2}, \mathrm{~L}_{3}$ y $\mathrm{L}_{\infty}$.

Para tener en cuenta más de un punto de vista de los responsables de la toma de decisiones, se han considerado diferentes pesos para las funciones objetivo, con el fin de darles más o menos importancia. Este análisis es de mucha utilidad para el establecimiento de escenarios que podrían darse en situaciones reales. Aunque los centros decisores de un gobierno busquen el desarrollo sostenible, es decir, el equilibrio entre objetivos sociales, económicos, ambientales, etc., existirán contextos y circunstancias en las cuales tengan mayor preferencia solamente por uno o varios de ellos. En el caso de la presente tesis doctoral, los objetivos estudiados se podrían resumir en cuatro temas principales: i) Energías renovables $\left(\mathrm{F}_{1}\right)$, ii) Medio ambiente $\left(\mathrm{F}_{2}, \mathrm{~F}_{3}\right.$ y $\left.\mathrm{F}_{4}\right)$, iii) Costes $\left(\mathrm{F}_{5}\right)$ y iv) Comunidades rurales aisladas $\left(\mathrm{F}_{6}\right)$. Según lo mencionado, en un contexto donde la política energética este orientada decididamente hacia la introducción de las energías renovables, se podría asignar un peso superior a la función respectiva frente a las otras. De igual forma, si lo que se busca de manera prioritaria es la reducción de emisiones de GEI para estar alineado con los compromisos asumidos en la lucha contra el cambio climático, se deberá asignar un peso mayor a cada una de las funciones ambientales. En otro contexto, con el fin de cerrar la brecha energética existente debido al bajo acceso a la energía en las CRA, los centros decisores tendrán preferencia por impulsar el acceso universal a la energía en esas comunidades, asignando un peso superior a este tema en el desarrollo de la planificación. Por último, si en la planificación se consideran prioritarios los costes, se actuaría de manera similar.

De acuerdo a lo mencionado y con el fin de considerar variedad de casos para analizar la tendencia de las variables, se han establecido dos escenarios para cada función objetivo. En el primero de ellos la función es el único criterio de interés para los centros decisores, para el caso de $\mathrm{F}_{1}:(1,0,0,0,0,0)$. En el otro, la función tiene un peso mayor que los demás, para el caso de $\mathrm{F}_{1}:(5,1,1,1,1,1)$. También se estableció un escenario base, es decir, dando a todas las funciones el mismo peso: $(1,1,1,1,1,1)$. 
Asimismo, se analizaron dos escenarios adicionales. Uno en la que se da un mayor peso a las funciones ambientales $\left(\mathrm{F}_{2}, \mathrm{~F}_{3}\right.$ y $\left.\mathrm{F}_{4}\right)$ y el otro, en la que el peso prioritario es asignado a las funciones sobre energías renovables y las $\mathrm{CRA}\left(\mathrm{F}_{1}\right.$ y $\left.\mathrm{F}_{6}\right)$. En la Tabla 6-1 se presentan los escenarios considerados según la asignación de pesos realizada.

Tabla 6-1. Establecimiento de escenarios según asignación de pesos a las funciones objetivo.

\begin{tabular}{lccccccc}
\hline \multicolumn{1}{c}{$\begin{array}{c}\text { Importancia de } \\
\text { objetivos }\end{array}$} & $\mathbf{F}_{\mathbf{1}}$ & $\mathbf{F}_{\mathbf{2}}$ & $\mathbf{F}_{\mathbf{3}}$ & $\mathbf{F}_{\mathbf{4}}$ & $\mathbf{F}_{\mathbf{5}}$ & $\mathbf{F}_{\mathbf{6}}$ & Escenario \\
\hline Igual importancia & 1 & 1 & 1 & 1 & 1 & 1 & 1 \\
\hline & 1 & 0 & 0 & 0 & 0 & 0 & 2 \\
& 5 & 1 & 1 & 1 & 1 & 1 & 3 \\
& 0 & 1 & 0 & 0 & 0 & 0 & 4 \\
Mayor o única & 1 & 5 & 1 & 1 & 1 & 1 & 5 \\
importancia & 0 & 0 & 1 & 0 & 0 & 0 & 6 \\
& 1 & 1 & 5 & 1 & 1 & 1 & 7 \\
& 0 & 0 & 0 & 1 & 0 & 0 & 8 \\
& 1 & 1 & 1 & 5 & 1 & 1 & 9 \\
& 0 & 0 & 0 & 0 & 1 & 0 & 10 \\
& 1 & 1 & 1 & 1 & 5 & 1 & 11 \\
& 0 & 0 & 0 & 0 & 0 & 1 & 12 \\
Mayor importancia de & 1 & 5 & 5 & 5 & 1 & 1 & 14 \\
\hline & 5 & 1 & 1 & 1 & 1 & 5 & 15 \\
\hline
\end{tabular}

\subsection{Análisis de la variación de las alternativas energéticas para las distancias calculadas.}

\subsubsection{Solar Térmica (ST)}

Esta variable alcanza sus mayores valores con la distancia Manhattan $\left(\mathrm{L}_{1}\right)$. Cuando se potencia sólo la función de costes, ST alcanza su valor mínimo (0,1 ktep). Por otro lado, fue favorecida al dar importancia única a las funciones ambientales, a la función de energías renovables y a la de acceso universal a la energía, alcanzado un valor de 1,658 ktep.

Por otro lado, al dar mayor peso a las funciones de energías renovables y de acceso universal a la energía (Escenario: 5,1,1,1,1,5), se observa que la variable se potencia. 


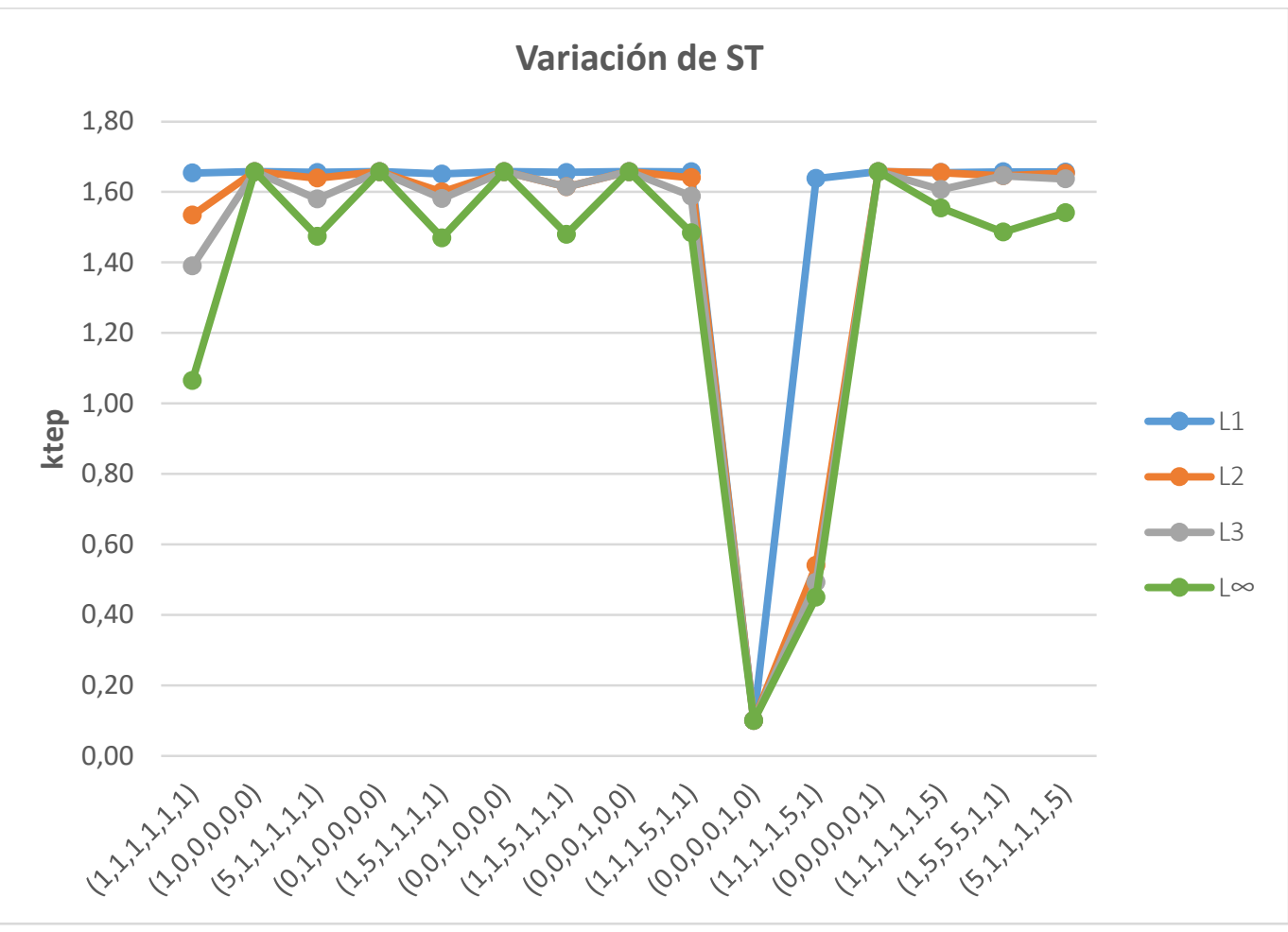

Figura 6-1. Variación de ST según los escenarios estudiados y para las distancias $\mathrm{L}_{1}, \mathrm{~L}_{2}, \mathrm{~L}_{3}$ y $\mathrm{L}_{\infty}$.

\subsubsection{Solar Fotovoltaica (PV)}

PV alcanza sus mayores valores con la distancia Manhattan $\left(\mathrm{L}_{1}\right)$, llegando a un valor máximo de 3,355 ktep al tener como único criterio el suministro de energía en las CRA $\left(\mathrm{F}_{6}\right)$.

El valor mínimo lo alcanza al tomar como criterios únicos las funciones ambientales $(0,1,1,1,0,0)$ y la función de costes $(0,0,0,0,1,0)$, dicho valor fue de 2,644 ktep en todos los casos.

Esta variable es claramente potenciada al dar un mayor peso a las funciones de energías renovables $\left(\mathrm{F}_{1}\right)$ y de acceso universal a la energía $\left(\mathrm{F}_{6}\right)$.

Es de destacar que el límite inferior de PV fue fijado en 2,644 ktep con el fin de asegurar el abastecimiento de energía eléctrica a la minera Yanacocha a través de esta fuente energética. 


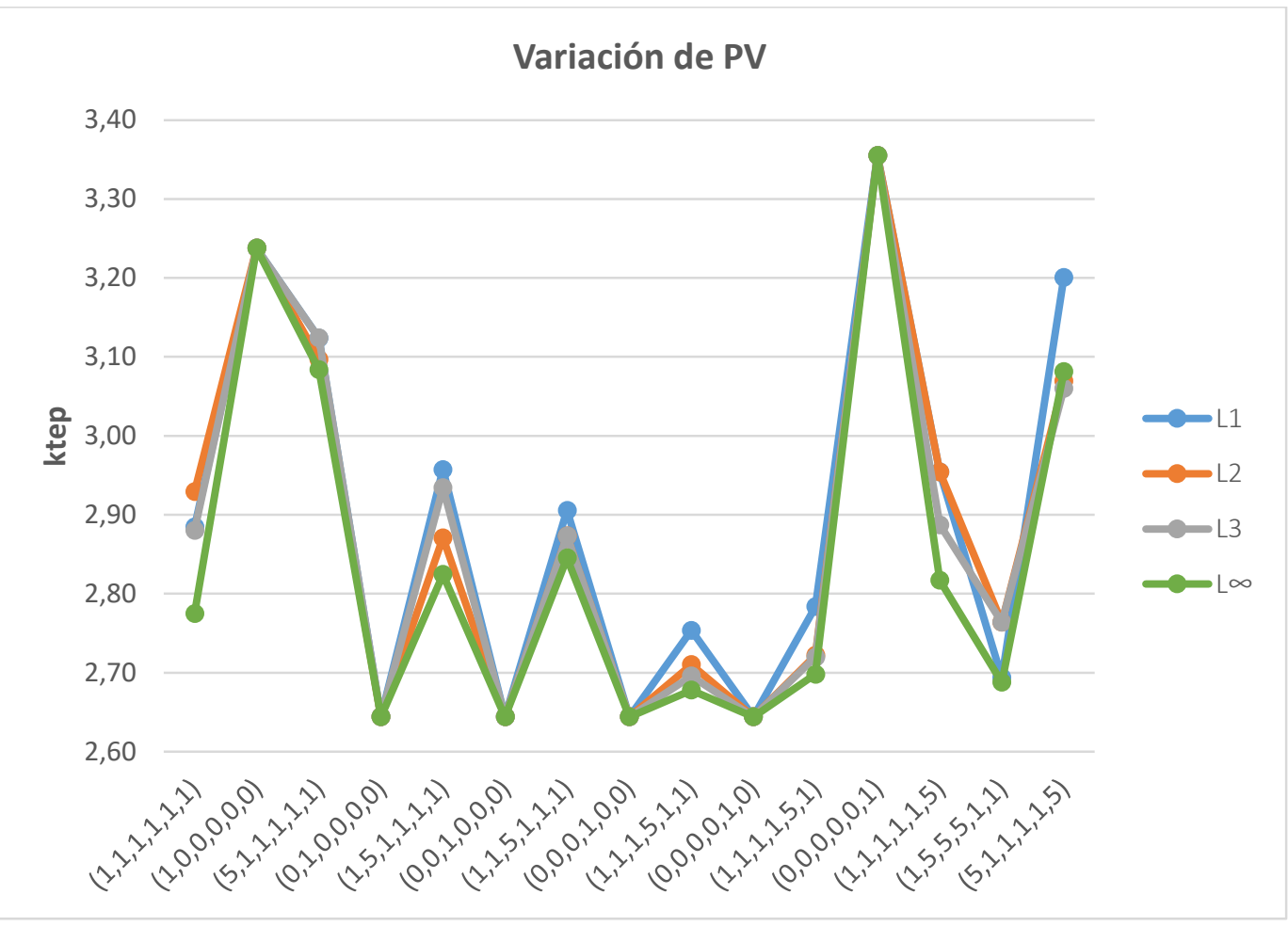

Figura 6-2. Variación de PV según los escenarios estudiados y para las distancias $\mathrm{L}_{1}, \mathrm{~L}_{2}, \mathrm{~L}_{3}$ y $\mathrm{L}_{\infty}$.

\subsubsection{Energía Eólica (EOL)}

Esta variable alcanza su valor mínimo cuando se prioriza de forma única la función de energías renovables, dicho valor fue de 0,013 ktep. Por otro lado, alcanza su valor máximo (2,393 ktep) al priorizar las funciones ambientales $F_{2}$ y $F_{3}$, y la función de acceso universal a la energía $\left(\mathrm{F}_{6}\right)$.

Con respecto a la priorización de las funciones ambientales frente a las de energías renovables y acceso universal a la energía, al dar mayor peso a las primeras $(1,5,5,5,1,1)$, EOL resultó más favorecida que al dar mayor peso a las dos últimas $(5,1,1,1,1,5)$. Se obtuvieron los mayores valores con la distancia Manhattan $\left(\mathrm{L}_{1}\right)$ para ambos casos. 


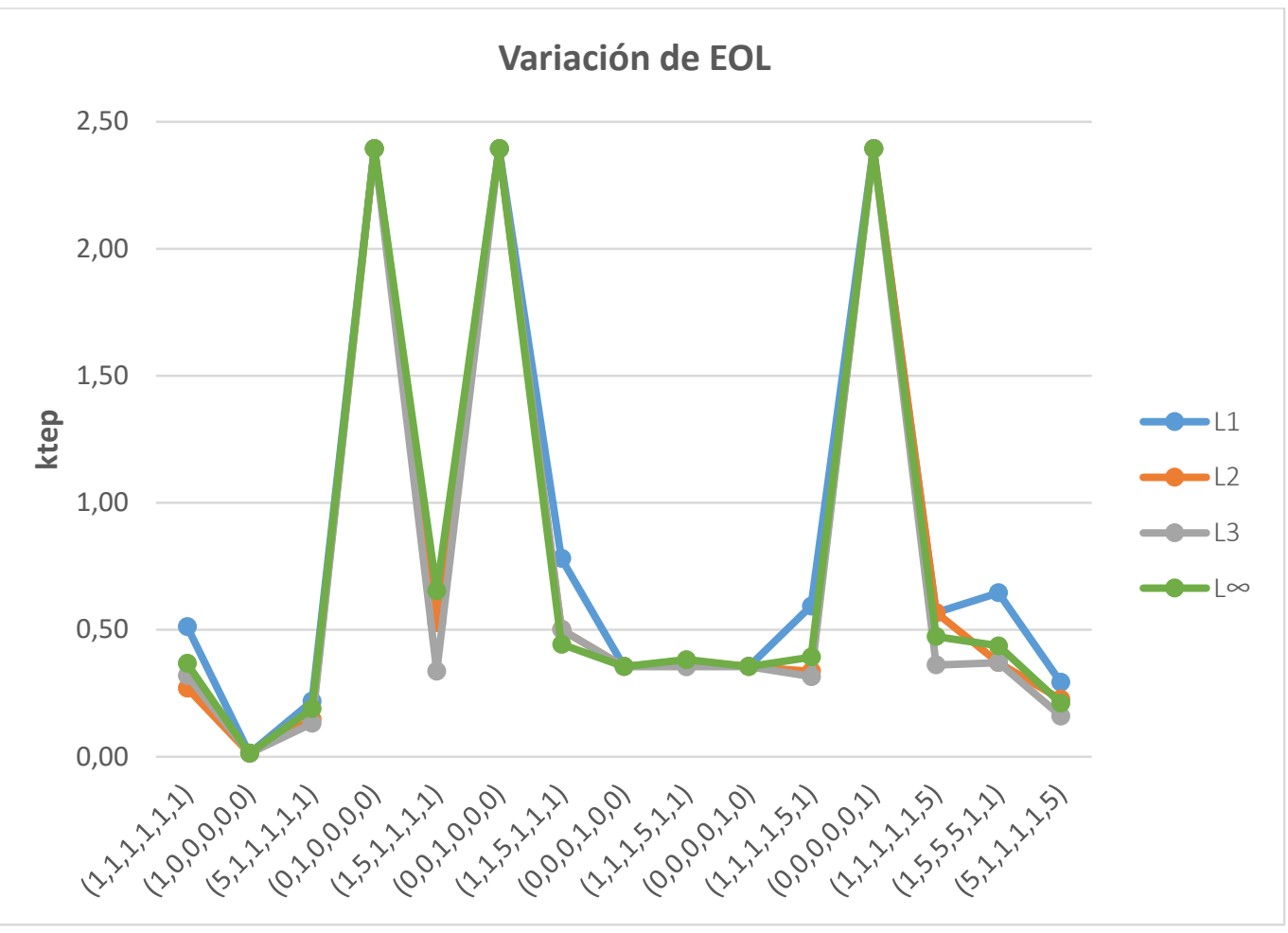
$\mathrm{L}_{\infty}$.

Figura 6-3. Variación de EOL según los escenarios estudiados y para las distancias $\mathrm{L}_{1}, \mathrm{~L}_{2}, \mathrm{~L}_{3}$ y

\subsubsection{Leñas (LEÑ)}

Se obtuvieron los mayores valores con la distancia Manhattan $\left(\mathrm{L}_{1}\right)$. El valor mínimo $(1,7$ ktep) se obtuvo al tener cómo único criterio la función de costes.

Se observa que LEÑ se ve favorecida al considerar como criterio único a las funciones ambientales. Esta tendencia se observa mucho más pronunciada en la distancia Chebyshev $\left(\mathrm{L}_{\infty}\right)$. Lo mismo ocurre al considerar la función de energías renovables y la función de acceso universal a la energía como criterios únicos, la variable LEÑ es potenciada. Para los escenarios mencionados el valor de la variable es constante (12,693 ktep).

Para los demás escenarios, los valores no son inferiores a 11 ktep, en la mayoría de los casos. 


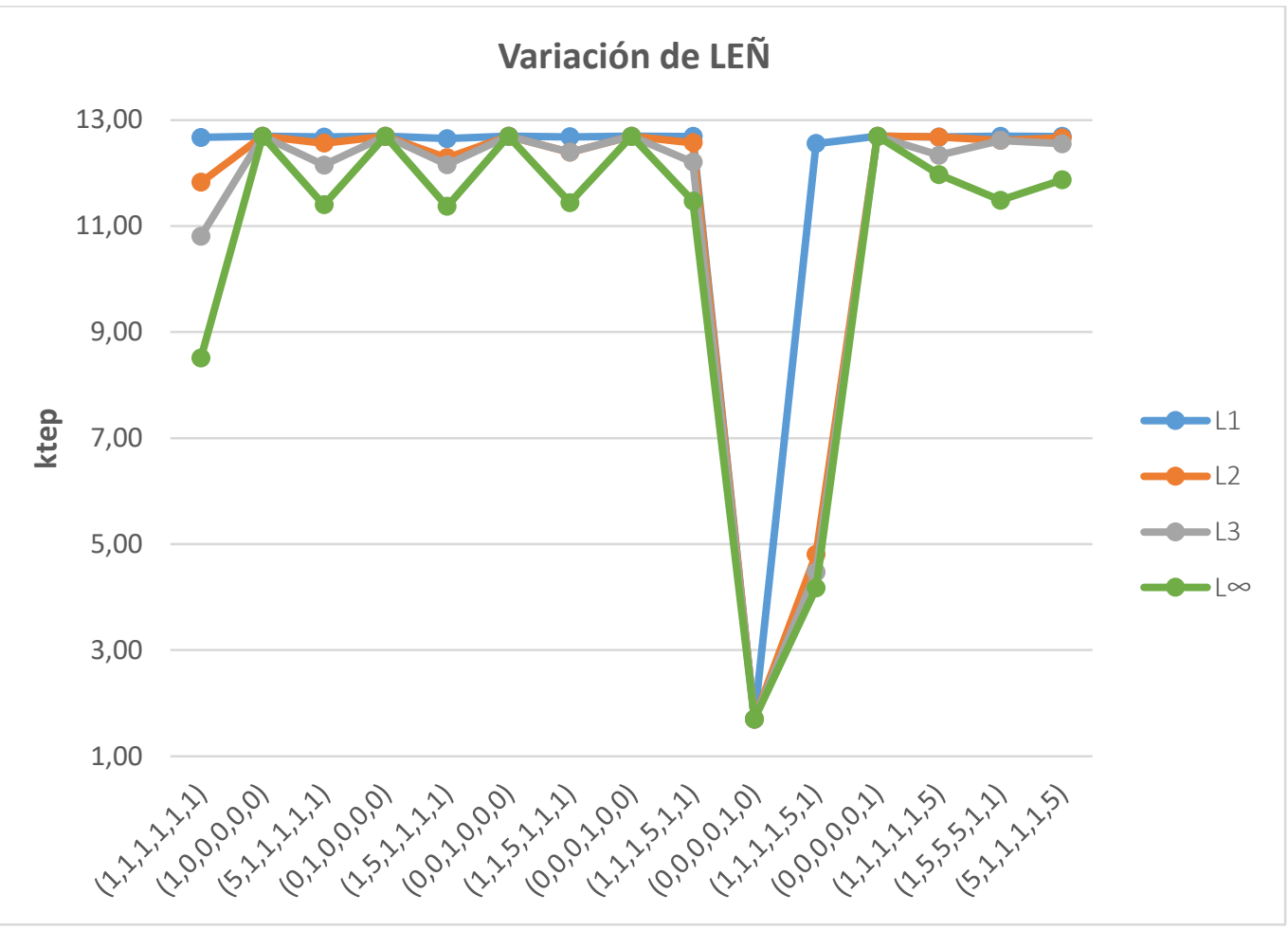
$\mathrm{L}_{\infty}$.

Figura 6-4. Variación de LEÑ según los escenarios estudiados y para las distancias $\mathrm{L}_{1}, \mathrm{~L}_{2}, \mathrm{~L}_{3}$ y

\subsubsection{Carbón Vegetal (CV)}

La tendencia de esta variable es semejante a LEÑ. La distancia Manhattan $\left(\mathrm{L}_{1}\right)$ se muestra casi constante en todos los escenarios, excepto en los casos donde se prioriza la función de costes $(0,0,0,0,1,0)$ y $(1,1,1,1,5,1)$, siendo el valor mínimo alcanzado de 1,0 ktep en el primer caso.

En las distancias $\mathrm{L}_{2}, \mathrm{~L}_{3}$ y $\mathrm{L}_{\infty}$, la tendencia es potenciar la variable cuando se da prioridad única a las funciones ambientales, llegando a un valor máximo de 2,705 ktep. 


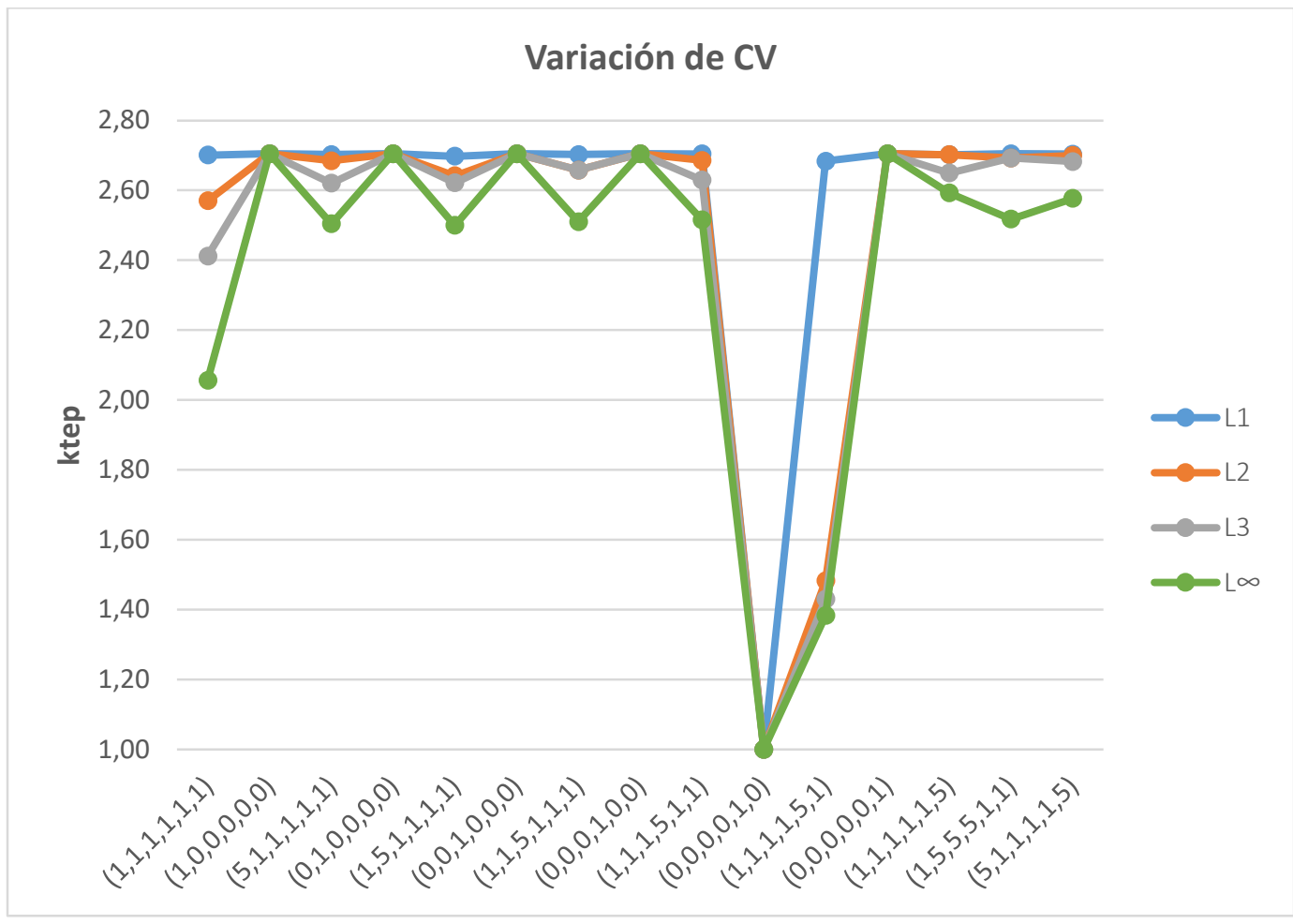

Figura 6-5. Variación de CV según los escenarios estudiados y para las distancias $\mathrm{L}_{1}, \mathrm{~L}_{2}, \mathrm{~L}_{3}$ y $\mathrm{L}_{\infty}$.

\subsubsection{Biocombustibles Líquidos (BL)}

Como en las anteriores variables, BL alcanza sus valores mínimos con la función de minimización de costes $\left(\mathrm{F}_{5}\right)$, siendo el valor mínimo 0,74 ktep cuando se considera a dicha función como criterio único $(0,0,0,0,1,0)$.

Es interesante notar que, dentro de las funciones ambientales, la de maximización de emisiones evitadas de $\mathrm{NO}_{\mathrm{x}}\left(\mathrm{F}_{3}\right)$, cuando es la única prioridad, disminuye la variable BL, alcanzando el valor de 0,887 ktep en todas las distancias consideradas. Esto se debe a que, para dicha función, no se tuvo en cuenta las emisiones evitadas de $\mathrm{NO}_{\mathrm{x}}$ por los motivos mencionados el apartado 5.3.2 del Capítulo V.

Cabe destacar que se ha fijado el valor mínimo de BL teniendo en cuenta cubrir la demanda del sector transporte, dicho valor fue de $0,74 \mathrm{ktep}$.

Como era de esperarse, hay una tendencia a potenciar esta variable cuando tienen mayor prioridad las funciones ambientales $(1,5,5,5,1,1)$, y las de energías renovables y acceso universal a la energía $(5,1,1,1,1,5)$. 


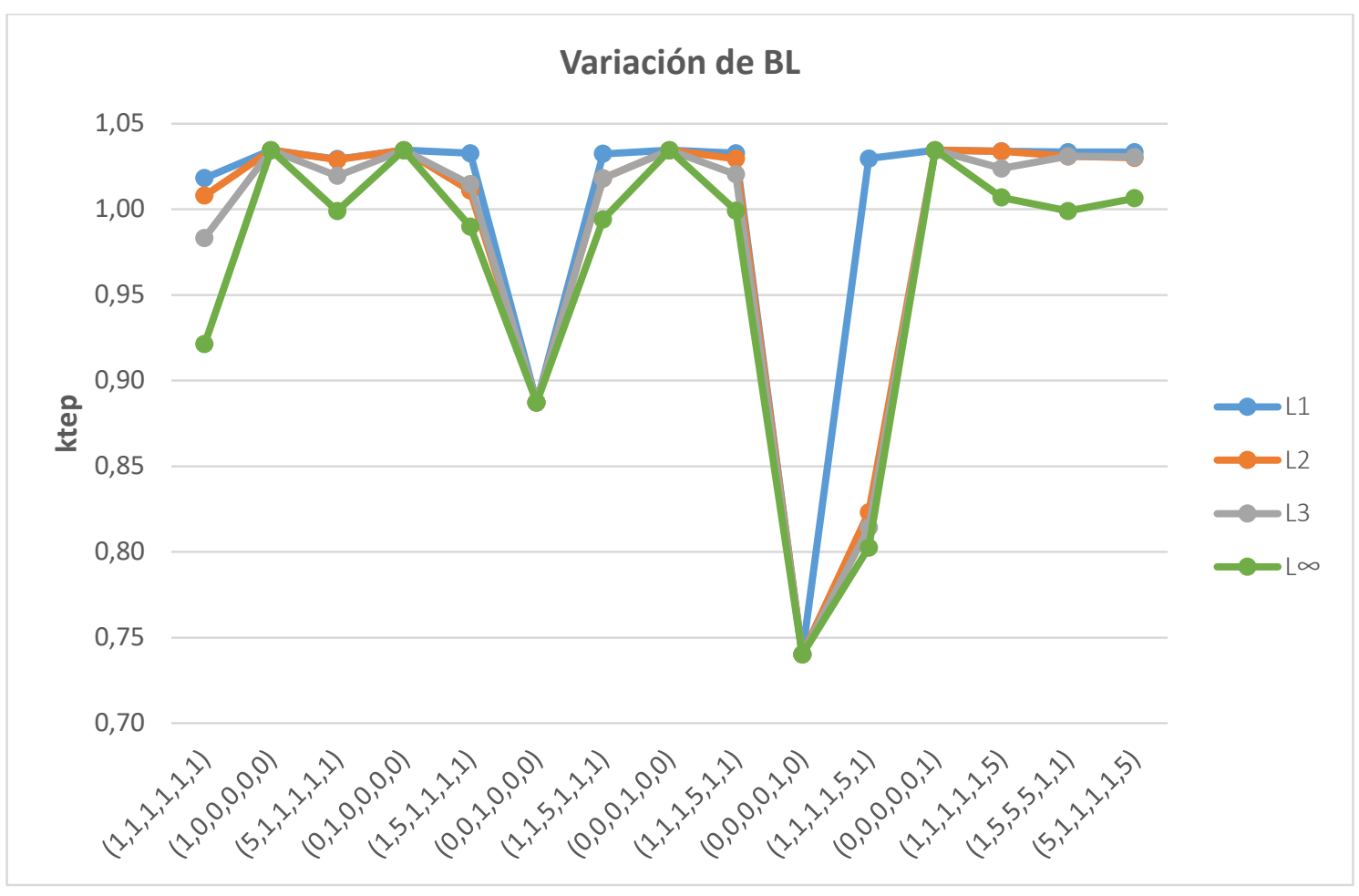

Figura 6-6. Variación de BL según los escenarios estudiados y para las distancias $\mathrm{L}_{1}, \mathrm{~L}_{2}, \mathrm{~L}_{3}$ y $\mathrm{L}_{\infty}$.

\subsubsection{Mini Hidráulica (MH)}

Esta variable se favorece cuando se priorizan las funciones ambientales independientemente, frente a las demás funciones: $(1,5,1,1,1,1),(1,1,5,1,1,1) \mathrm{y}$ $(1,1,1,5,1,1)$, lo cual se refleja también al asignar un mayor peso a las tres en su conjunto frente a las otras: $(1,5,5,5,1,1)$. Según esto, se podría decir que $\mathrm{MH}$ es la variable que se potencia más al favorecer las funciones ambientales.

El mayor valor de $\mathrm{MH}$ se alcanza al dar prioridad única a la función de emisiones evitadas de $\mathrm{SO}_{2}\left(\mathrm{~F}_{4}\right)$ y a la función de costes, llegando a un valor de 3,725 ktep. Por otro lado, el menor valor es alcanzado al priorizar la función de acceso universal a la energía $(0,976 \mathrm{ktep})$.

Es relevante notar que la variable $\mathrm{MH}$ es la que más se favorece priorizar la función de costes. 


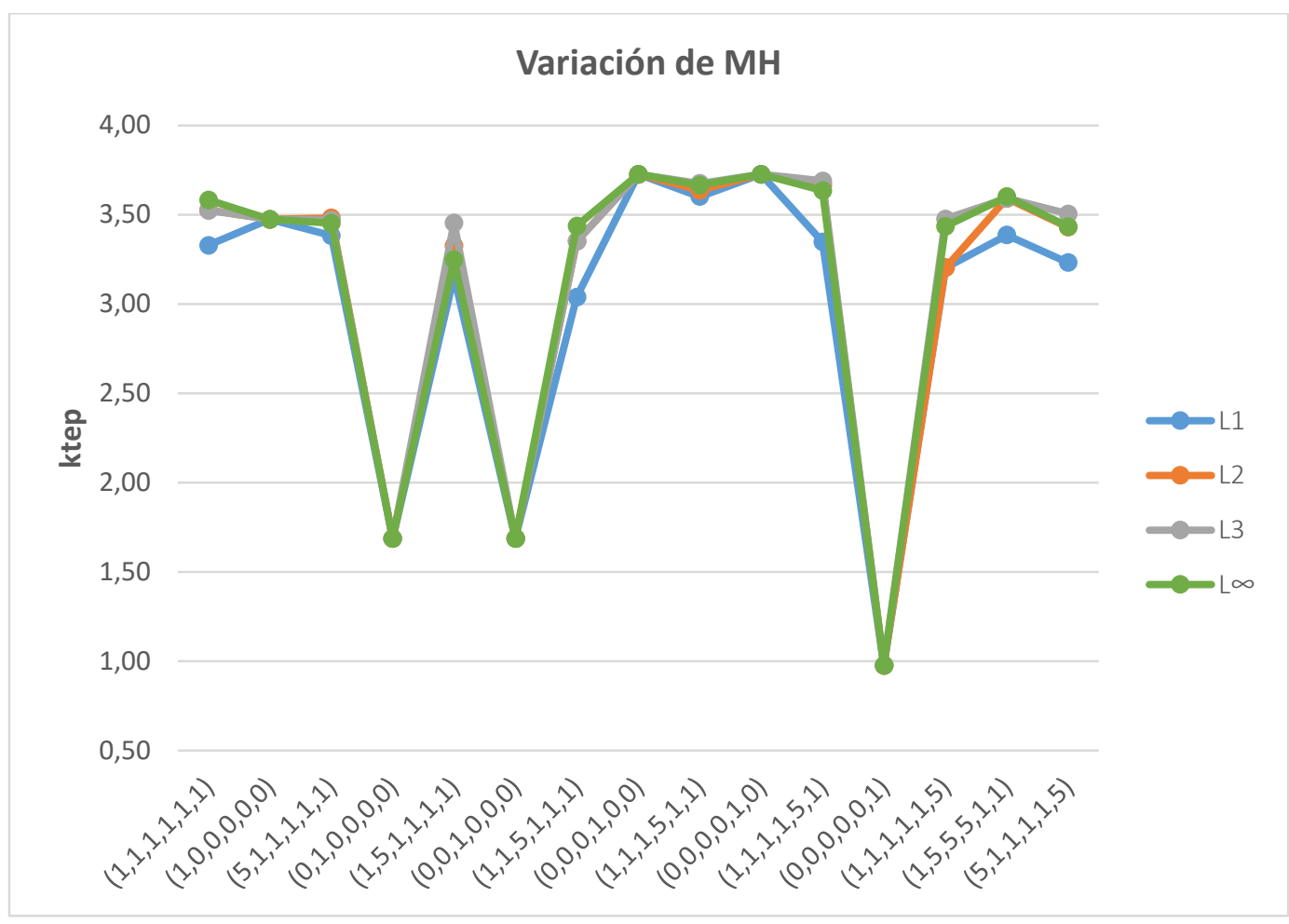

Figura 6-7. Variación de $\mathrm{MH}$ según los escenarios estudiados y para las distancias $\mathrm{L}_{1}, \mathrm{~L}_{2}, \mathrm{~L}_{3}$ y $\mathrm{L}_{\infty}$.

\subsubsection{Geotérmica (GEO)}

El comportamiento de las distancias de esta variable es similar al obtenido para ST, LEÑ y CV, aunque el rango de variación es claramente menor, alcanzando valores de 0,05 a 0,205 ktep.

La variable se observa ligeramente favorecida al priorizar las funciones ambientales como criterio único, llegando en estos casos a alcanzar su valor máximo $(0,205$ ktep). Cuando la función de coste es priorizada alcanza sus valores mínimos $(0,05$ y 0,085 ktep). 


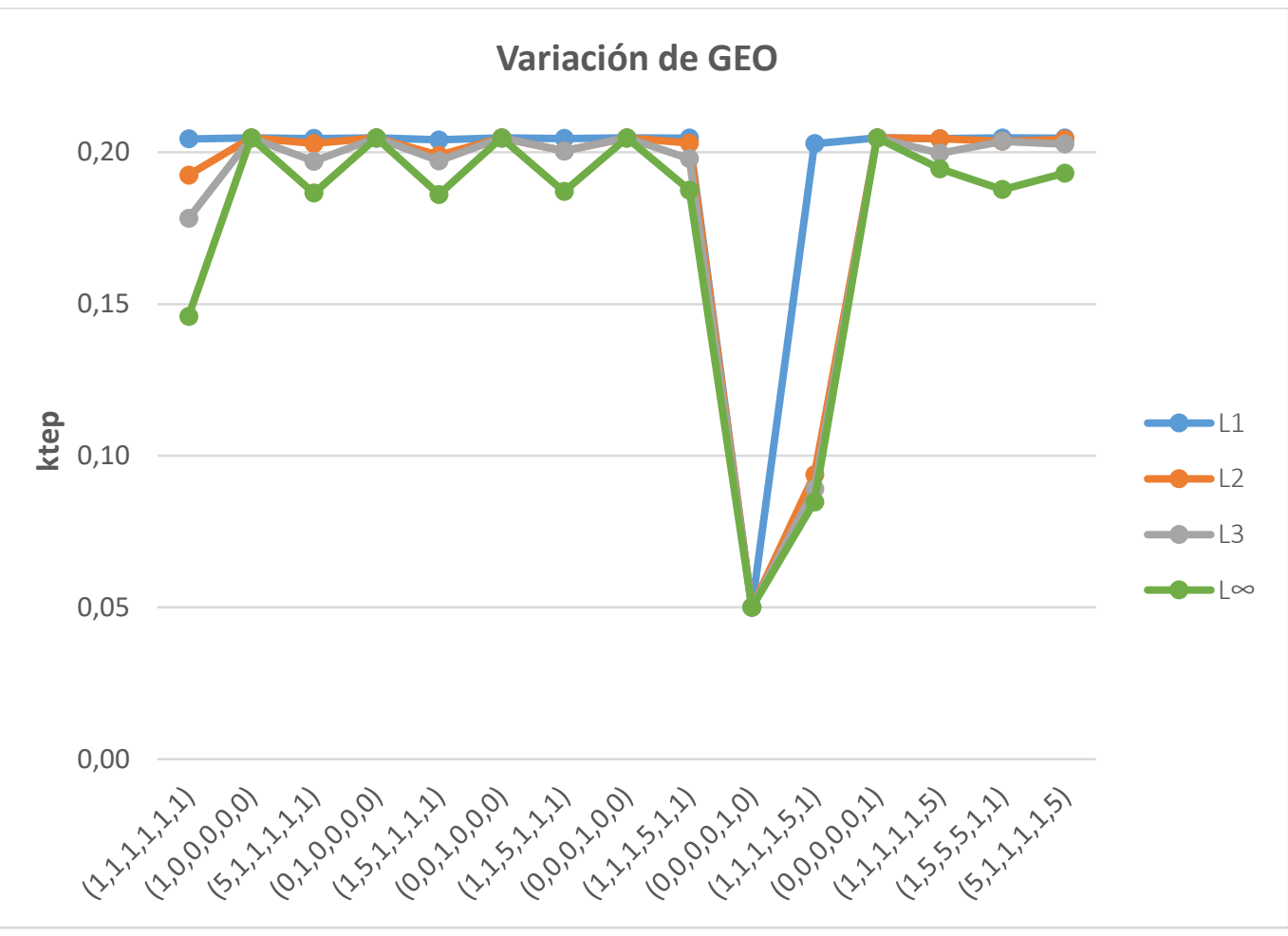
$\mathrm{L}_{\infty}$.

Figura 6-8. Variación de GEO según los escenarios estudiados y para las distancias $L_{1}, L_{2}, L_{3}$ y

\subsection{Análisis de la variación de las funciones objetivo para las distancias calculadas.}

A continuación, se presentan los resultados de la variación de las seis funciones objetivo consideradas en el presente estudio. Se observa en todos los casos el efecto de la función de minimización de costes $\left(\mathrm{F}_{5}\right)$, en el que, como era de esperarse, las funciones tienden a alcanzar sus valores mínimos. Por ello, en el análisis realizado en los siguientes apartados, no se ha considerado dichos valores. Por otro lado, se pudo comprobar que las funciones de maximización alcanzaban sus valores máximos al considerarlas como criterio único, casos $(1,0,0,0,0,0),(0,1,0,0,0,0)$, $(0,0,1,0,0,0),(0,0,0,1,0,0)$ y $(0,0,0,0,0,1)$.

\subsubsection{F1: Maximización de la sustitución de las energías fósiles por energías renovables.}

El valor máximo alcanzado en todas las distancias fue de 44,661 ktep. 
Las distribuciones que en promedio alcanzaron los valores más altos fueron: $(0,0,0,1,0,0),(1,1,1,1,1,5)$ y $(5,1,1,1,1,5)$. Esto indica que al priorizar las funciones $\mathrm{F}_{4} \mathrm{y} \mathrm{F}_{6}$, se favorece a la función $\mathrm{F}_{1}$.

Por otro lado, los valores más bajos fueron obtenidos con las siguientes distribuciones de pesos: $(1,1,1,1,1,1),(1,5,1,1,1,1)$ y $(0,0,1,0,0,0)$. Esto quiere decir que la optimización no ponderada, la función de emisiones de $\mathrm{CO}_{2}$ y la función de emisiones de $\mathrm{NO}_{\mathrm{x}}$ no contribuyen a la maximización de $\mathrm{F}_{1}$.

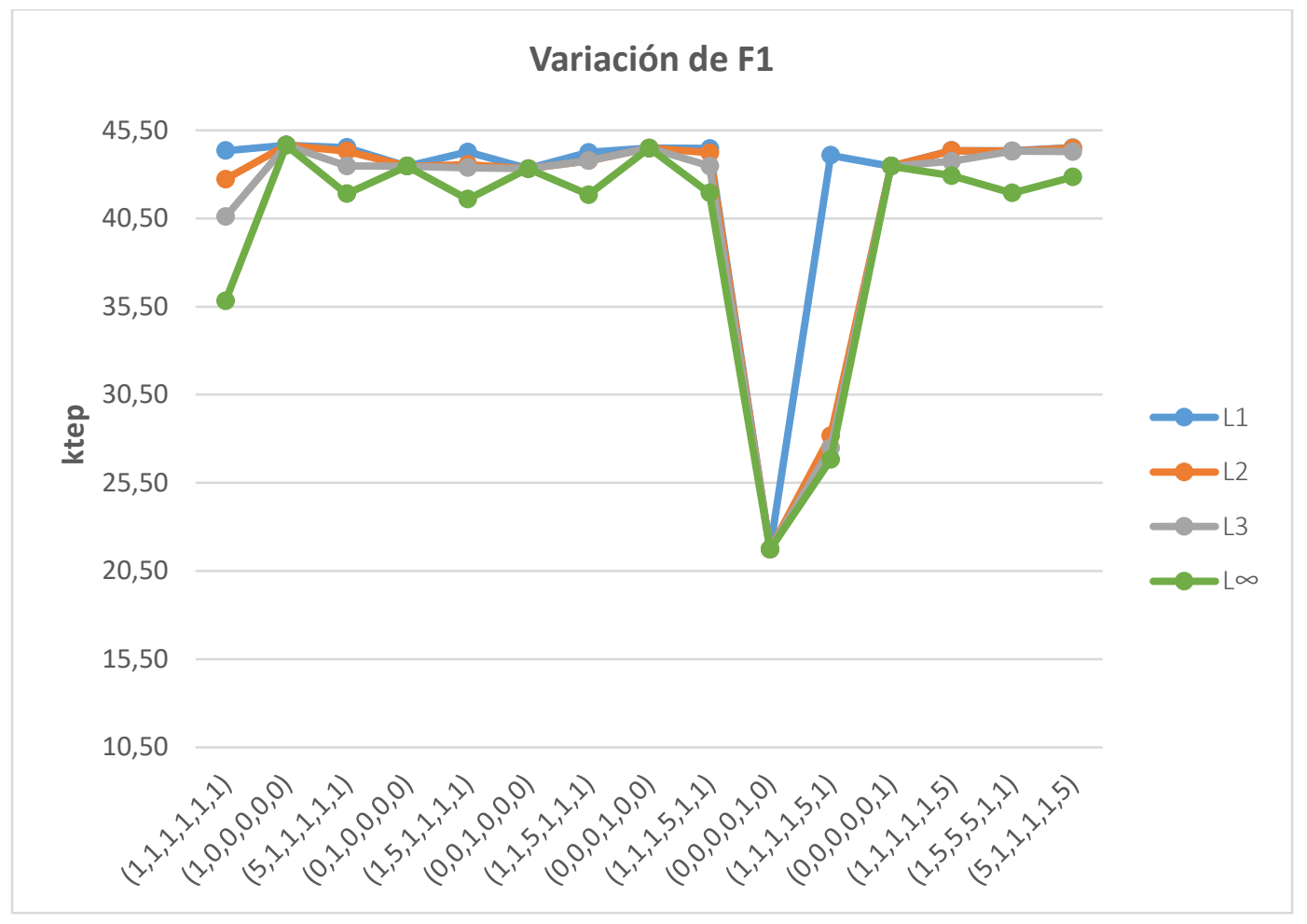

Figura 6-9. Variación de $F_{1}$ según los escenarios estudiados y para las distancias $L_{1}, L_{2}, L_{3}$ y $L_{\infty}$.

\subsubsection{F2: Maximización de las emisiones evitadas de $\mathrm{CO}_{2}$.}

El máximo valor alcanzado para esta función fue de $163,931 \mathrm{ktCO}_{2}$.

Los valores más altos fueron alcanzados con las distribuciones de pesos $(1,0,0,0,0,0), \quad(0,0,1,0,0,0)$ y $(0,0,0,1,0,0)$. Es decir, que la priorización independiente de las funciones $\mathrm{F}_{1}, \mathrm{~F}_{3} \mathrm{y} \mathrm{F}_{4}$, favorecieron la función de emisiones evitadas de $\mathrm{CO}_{2}$. 
Las distribuciones de pesos $(1,1,1,1,1,1),(5,1,1,1,1,1)$ y $(1,5,1,1,1,1)$ fueron los que obtuvieron los valores mínimos para la función analizada. El primer caso indica que la optimización no ponderada no favorece a la función $\mathrm{F}_{2}$, lo mismo ocurre al darle mayor importancia a $F_{1}$. Es interesante destacar cómo en el tercer caso se obtienen valores bajos incluso cuando se prioriza la misma función $F_{2}$. Eso significa que la distribución no ponderada está efectivamente muy fuertemente dirigida hacia bajas emisiones evitadas de $\mathrm{CO}_{2}$.

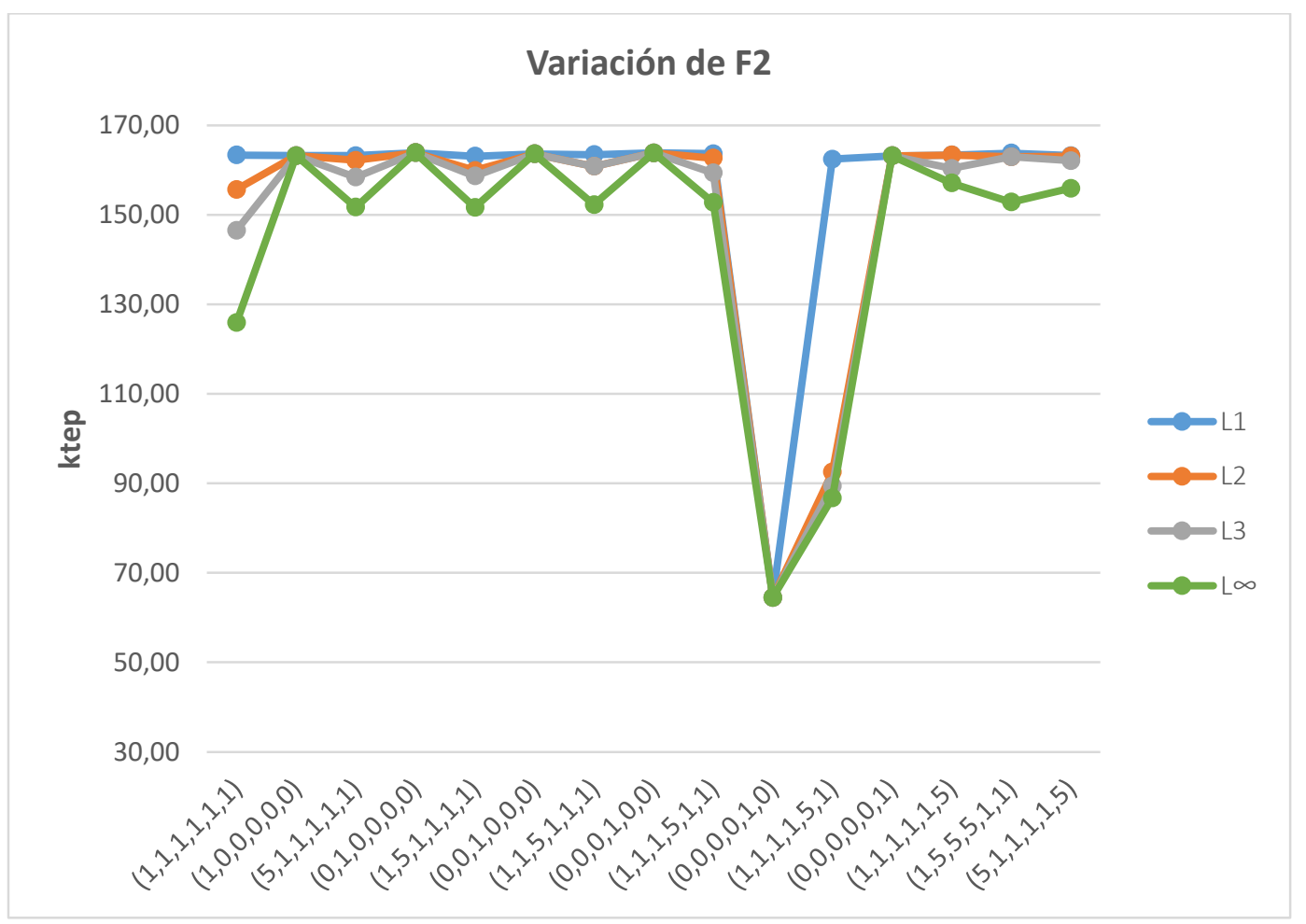

Figura 6-10. Variación de $\mathrm{F}_{2}$ según los escenarios estudiados y para las distancias $\mathrm{L}_{1}, \mathrm{~L}_{2}, \mathrm{~L}_{3}$ y $\mathrm{L}_{\infty}$.

\subsubsection{F3: Maximización de las emisiones evitadas de NOx.}

El valor máximo de esta función fue de 0,41 ktNO . Además de la distribución donde se maximiza dicha función como criterio único, también se obtuvo dicho valor para las siguientes distribuciones: $(0,1,0,0,0,0)$ y $(0,0,0,1,0,0)$, esto quiere decir que al priorizar las funciones de emisiones de $\mathrm{CO}_{2}$ y $\mathrm{SO}_{2}\left(\mathrm{~F}_{2}\right.$ y $\left.\mathrm{F}_{4}\right)$, también estamos priorizando la función $\mathrm{F}_{3}$.

Los valores mínimos fueron obtenidos con las distribuciones $(1,1,1,1,1,1)$, $(5,1,1,1,1,1)$ y $(1,5,1,1,1,1)$. El primer escenario quiere decir que la función de 
optimización no ponderada no contribuye a la maximización de emisiones evitadas de $\mathrm{NO}_{\mathrm{x}}$. El segundo y tercer escenario quieren decir que las funciones $\mathrm{F}_{1}$ y $\mathrm{F}_{2}$ cuando son priorizadas independientemente frente a las otras funciones, como 5 es a 1, no favorecen a las emisiones evitadas de $\mathrm{NO}_{\mathrm{X}}$.

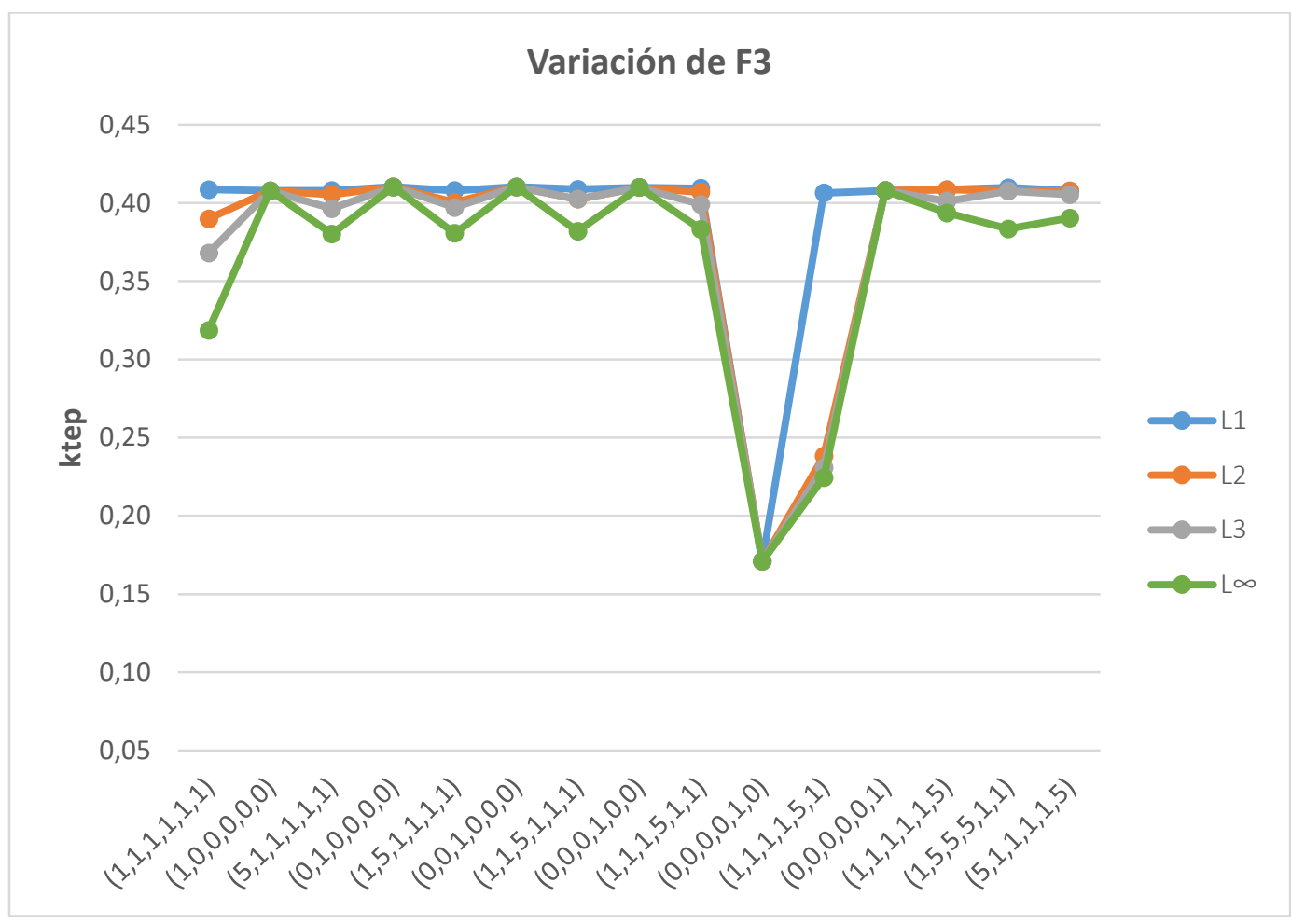

Figura 6-11. Variación de $\mathrm{F}_{3}$ según los escenarios estudiados y para las distancias $\mathrm{L}_{1}, \mathrm{~L}_{2}, \mathrm{~L}_{3}$ y $\mathrm{L}_{\infty}$.

\subsubsection{F4: Maximización de las emisiones evitadas de $\mathrm{SO}_{2}$.}

El valor máximo de esta función fue de $0,688 \mathrm{ktSO}_{2}$. Además de la distribución donde se maximiza dicha función como criterio único, también se obtuvo dicho valor para la distribución $(0,1,0,0,0,0)$, esto quiere decir que al priorizar la función de emisiones de $\mathrm{CO}_{2}\left(\mathrm{~F}_{2}\right)$, también estamos priorizando la función $\mathrm{F}_{4}$. Los siguientes mejores valores se obtuvieron con $(1,0,0,0,0,0)$ y $(0,0,1,0,0,0)$, es decir, que la priorización de las funciones $\mathrm{F}_{1}$ y $\mathrm{F}_{3}$ favorecieron a la maximización de las emisiones evitadas de $\mathrm{SO}_{2}$.

Los valores mínimos de $\mathrm{F}_{4}$ fueron obtenidos con las distribuciones: $(1,1,1,1,1,1)$, $(5,1,1,1,1,1)$ y $(1,5,1,1,1,1)$, lo cual quiere decir que la priorización de la función de 
optimización no ponderada y de las funciones $\mathrm{F}_{1} \mathrm{y} \mathrm{F}_{2}$ no favorecieron a la función $\mathrm{F}_{4}$.

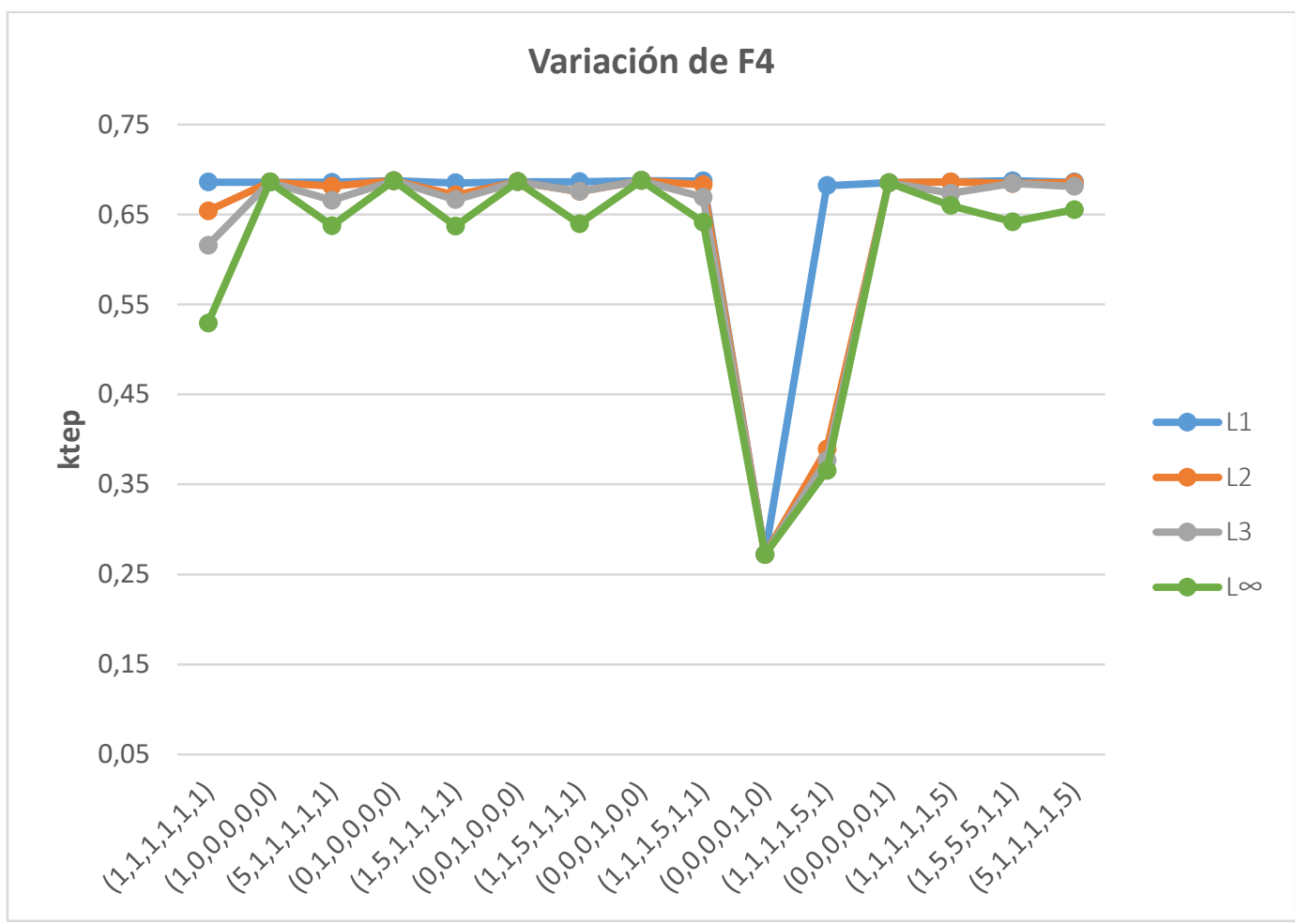

Figura 6-12. Variación de $\mathrm{F}_{4}$ según los escenarios estudiados y para las distancias $\mathrm{L}_{1}, \mathrm{~L}_{2}, \mathrm{~L}_{3}$ y $\mathrm{L}_{\infty}$.

\subsubsection{F5: Minimización de costes.}

En el análisis de esta función, al ser de minimización, se consideran como favorables los valores mínimos.

El mín mo valor alcanzado en todas las distancias fue de 16,50 USD ('000).

Los valores mínimos promedio se obtuvieron con las distribuciones $(1,1,1,1,1,1)$, $(0,0,0,1,0,0)$ y $(1,1,1,5,1,1)$. El primer caso indica que, al priorizar la función de optimización no ponderada, es decir, cuando todas las funciones tienen el mismo peso, se favorece la minimización de los costes. En el segundo y tercer escenario, es interesante destacar que cuando se optimiza independientemente la función de emisiones evitadas de $\mathrm{SO}_{2}$ y al darle más importancia que a otras, también se minimizan los costes. 
Por otro lado, los valores máximos fueron obtenidos con las distribuciones: $(0,1,0,0,0,0),(0,0,1,0,0,0)$ y $(0,0,0,0,0,1)$, lo cual significa que la priorización independiente de las funciones $\mathrm{F}_{2}, \mathrm{~F}_{3}$ y $\mathrm{F}_{6}$, fueron un obstáculo para la minimización de los costes.

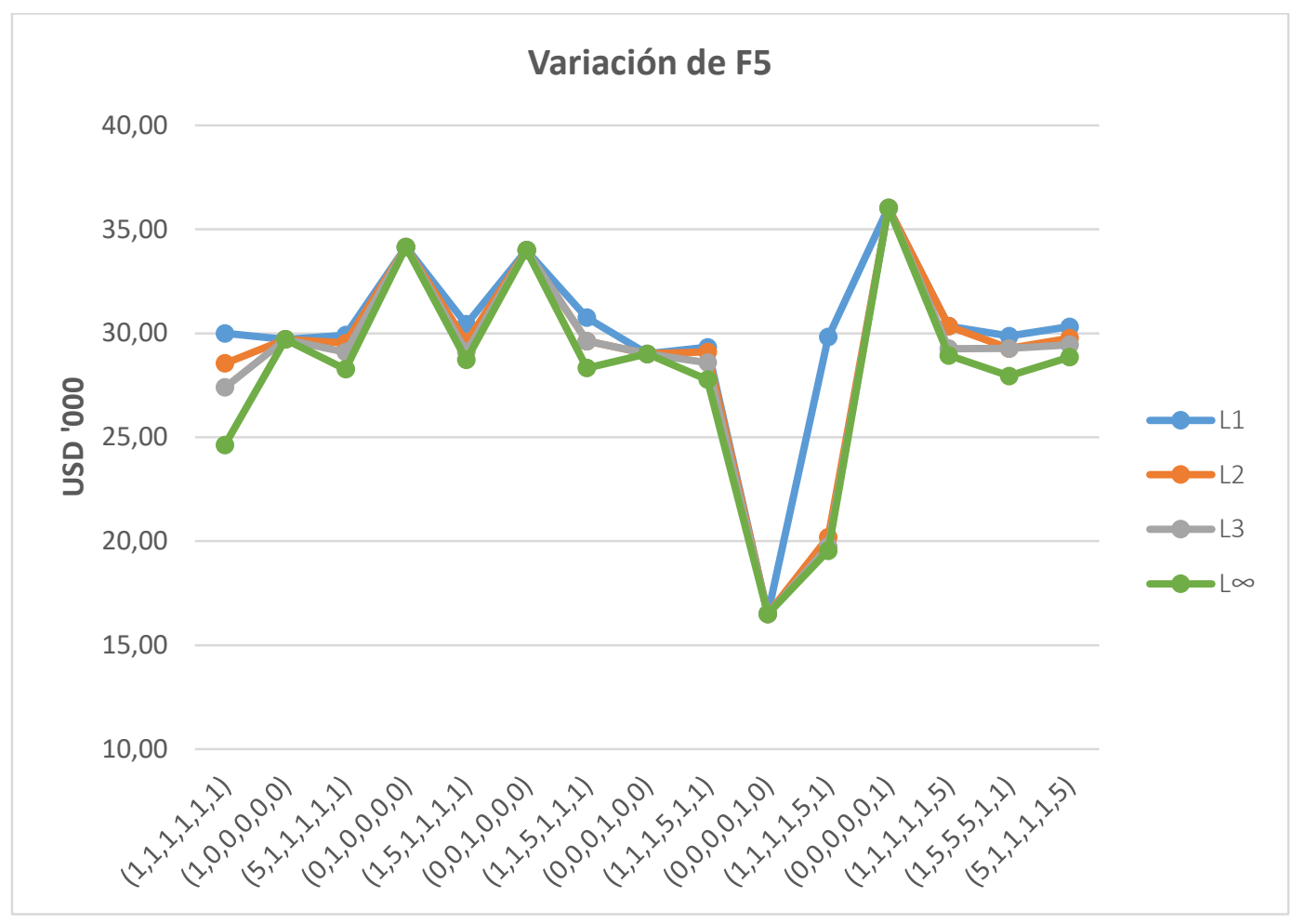

Figura 6-13. Variación de $\mathrm{F}_{5}$ según los escenarios estudiados y para las distancias $\mathrm{L}_{1}, \mathrm{~L}_{2}, \mathrm{~L}_{3}$ y $\mathrm{L}_{\infty}$.

\subsubsection{F6: Maximización del uso de alternativas energéticas renovables para el suministro sostenible de energía en CRA.}

El mayor valor obtenido para $\mathrm{F}_{6}$ fue de 42,290 ktep.

Las distribuciones de peso que obtuvieron los valores más altos para esta función, fueron: $(1,0,0,0,0,0),(0,1,0,0,0,0)$ y $(0,0,1,0,0,0)$. Esto quiere decir que la priorización independiente de las funciones $\mathrm{F}_{1}, \mathrm{~F}_{2}$ y $\mathrm{F}_{3}$, favoreció la maximización del uso de alternativas energéticas renovables para el suministro sostenible de energía en CRA.

Por otro lado, los valores mínimos se obtuvieron con las distribuciones $(1,1,1,1,1,1),(1,5,1,1,1,1)$ y $(1,1,1,5,1,1)$. El primer caso quiere decir que priorizar la función de optimización no ponderada no favorece la función $\mathrm{F}_{6}$. Los dos últimos 
casos quieren decir que al dar mayor prioridad a las funciones $\mathrm{F}_{2}$ y $\mathrm{F}_{4}$, como 5 es a 1, sobre las demás funciones, la función F6 no resulta favorecida.

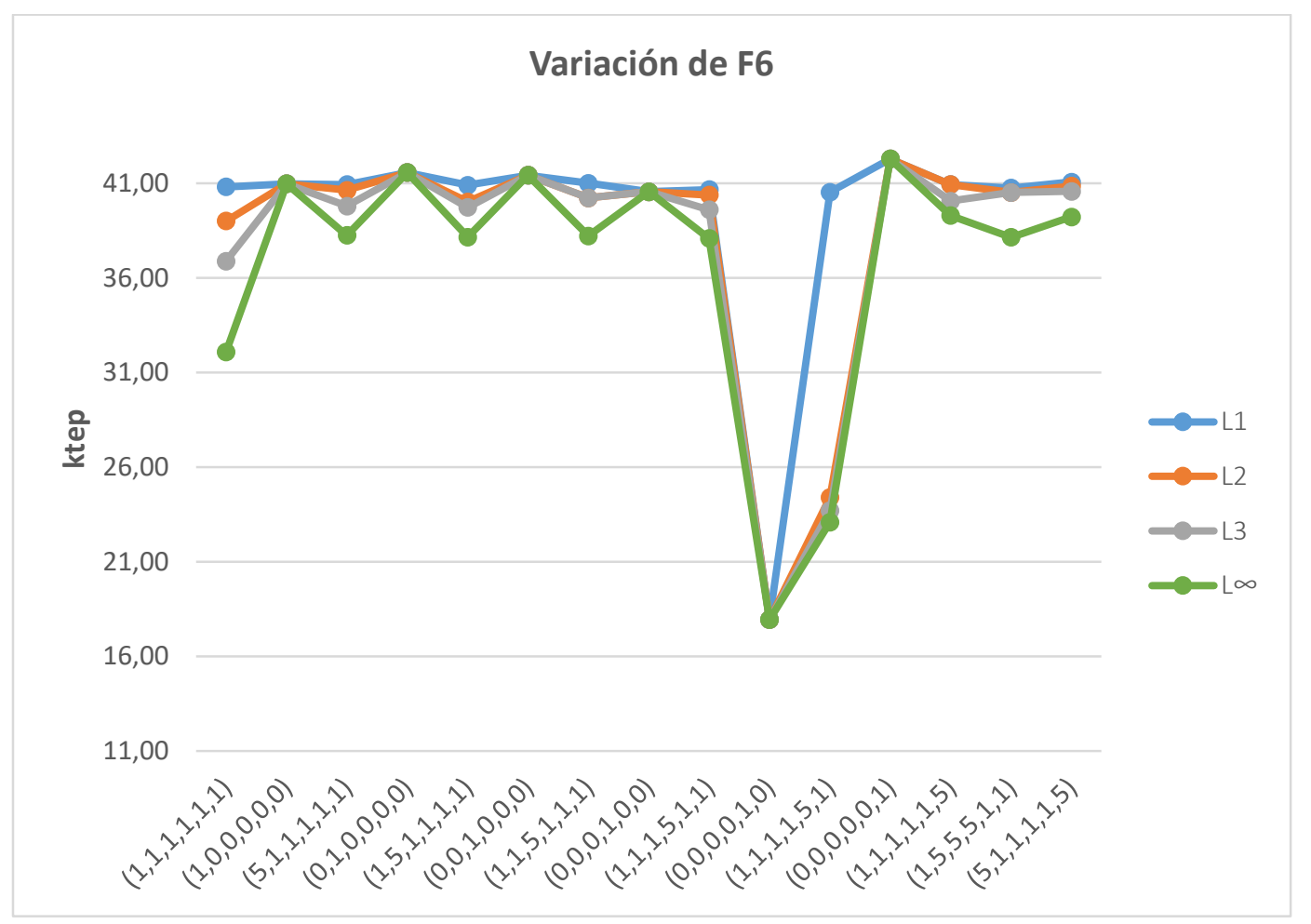

Figura 6-14. Variación de $\mathrm{F}_{6}$ según los escenarios estudiados y para las distancias $\mathrm{L}_{1}, \mathrm{~L}_{2}, \mathrm{~L}_{3}$ y $\mathrm{L}_{\infty}$.

\subsection{Análisis de la variación de las funciones objetivo para la distancia Chebyshev $(\mathbf{L} \infty)$.}

Entre las cuatro distancias calculadas se eligió la distancia de Chebyshev $\left(\mathrm{L}_{\infty}\right)$ como la solución óptima de Pareto, por representar la solución más equilibrada según Romero (1996).

La Tabla 6-1 muestra los resultados obtenidos para cada vector energético.

Tabla 6-2. Resultados obtenidos de la minimización de L $\infty$ para cada alternativa energética y considerando los escenarios estudiados (ktep).

\begin{tabular}{|c|c|c|c|c|c|c|c|c|c|c|c|c|c|}
\hline $\mathbf{F}_{1}$ & $F_{2}$ & & & $F_{5}$ & $F_{6}$ & ST & PV & EOL & LEÑ & $\mathrm{CV}$ & BL & MH & GEO \\
\hline 1 & 1 & 1 & 1 & 1 & 1 & 1,065 & 2,775 & 0,367 & 8,514 & 2,057 & 0,921 & 3,582 & 0,146 \\
\hline 1 & 0 & 0 & 0 & 0 & 0 & 1,658 & 3,238 & 0,013 & 12,693 & 2,705 & 1,034 & 3,474 & 0,205 \\
\hline 5 & 1 & 1 & 1 & 1 & 1 & 1,475 & 3,084 & 0,189 & 11,401 & 2,504 & 0,999 & 3,452 & 0,187 \\
\hline 0 & 1 & 0 & 0 & 0 & 0 & 1,658 & 2,644 & 2,393 & 12,693 & 2,705 & 1,034 & 1,687 & 0,205 \\
\hline 1 & 5 & 1 & 1 & 1 & 1 & 1,470 & 2,824 & 0,653 & 11,370 & 2,500 & 0,990 & 3,247 & 0,186 \\
\hline
\end{tabular}




\begin{tabular}{cccccccccccccc}
\hline \multicolumn{1}{c}{ Pesos } & \multicolumn{1}{c}{ ST } & PV & EOL & LEN & $\mathbf{C V}$ & $\mathbf{B L}$ & $\mathbf{M H}$ & $\mathbf{G E O}$ \\
\hline $\mathbf{F}_{\mathbf{1}}$ & $\mathbf{F}_{\mathbf{2}}$ & $\mathbf{F}_{\mathbf{3}}$ & $\mathbf{F}_{\mathbf{4}}$ & $\mathbf{F}_{\mathbf{5}}$ & $\mathbf{F}_{\mathbf{6}}$ & & & & & & & \\
\hline 0 & 0 & 1 & 0 & 0 & 0 & 1,658 & 2,644 & 2,393 & 12,693 & 2,705 & 0,887 & 1,687 & 0,205 \\
1 & 1 & 5 & 1 & 1 & 1 & 1,480 & 2,845 & 0,443 & 11,440 & 2,510 & 0,994 & 3,437 & 0,187 \\
0 & 0 & 0 & 1 & 0 & 0 & 1,658 & 2,644 & 0,355 & 12,693 & 2,705 & 1,034 & 3,725 & 0,205 \\
1 & 1 & 1 & 5 & 1 & 1 & 1,485 & 2,678 & 0,382 & 11,473 & 2,515 & 0,999 & 3,665 & 0,188 \\
0 & 0 & 0 & 0 & 1 & 0 & 0,100 & 2,644 & 0,355 & 1,700 & 1,000 & 0,740 & 3,725 & 0,050 \\
1 & 1 & 1 & 1 & 5 & 1 & 0,450 & 2,698 & 0,392 & 4,170 & 1,383 & 0,802 & 3,634 & 0,085 \\
0 & 0 & 0 & 0 & 0 & 1 & 1,658 & 3,355 & 2,393 & 12,693 & 2,705 & 1,034 & 0,976 & 0,205 \\
1 & 1 & 1 & 1 & 1 & 5 & 1,555 & 2,817 & 0,473 & 11,970 & 2,593 & 1,007 & 3,435 & 0,195 \\
1 & 5 & 5 & 5 & 1 & 1 & 1,486 & 2,688 & 0,436 & 11,485 & 2,517 & 0,999 & 3,601 & 0,188 \\
5 & 1 & 1 & 1 & 1 & 5 & 1,541 & 3,081 & 0,211 & 11,869 & 2,577 & 1,006 & 3,432 & 0,193 \\
\hline
\end{tabular}

Los resultados muestran que las alternativas energéticas que experimentaron mejoras según el peso asignado fueron ST, LEÑ, CV y GEO, en la mayoría de los casos. Mientras tanto, PV, EOL, BL y MH presentaron una mayor variación según los casos y fueron los menos favorecidos en la mayoría de ellos.

Se han representado gráficamente los resultados de la Tabla 6-1 (Figuras del 6-16 al 6-23). Con el fin de facilitar la comparativa con el escenario base (Figura 6-15) se ha incorporado el respectivo diagrama de barras en los gráficos analizados, los cuales se presentan según la priorización de cada función objetivo. Para facilitar la discusión de los resultados no se ha tenido en cuenta los valores alcanzados por la variable LEÑ, ya que en todos los casos se mantiene en niveles superiores a las demás fuentes energéticas renovables.

La Figura 6-20 muestra que, al considerar sólo la función de coste $\left(\mathrm{F}_{5}\right)$, los valores de las variables fueron mínimos, con excepción de las variables PV, MH y EOL, las cuales estuvieron condicionadas a alcanzar la demanda de energía eléctrica planificada. Se puede observar que, en los dos escenarios analizados, $\mathrm{MH}$ se convierte en la alternativa con mayor prioridad, asimismo, que la variable PV ocupa el segundo lugar, en ambos casos.

Con respecto a la variable PV, obtuvo los valores más bajos con la maximización independiente de las funciones ambientales $\left(\mathrm{F}_{2}, \mathrm{~F}_{3}\right.$ y $\left.\mathrm{F}_{4}\right)$. El valor más alto se obtuvo al considerar sólo la función $\mathrm{F}_{6}$ (Figura 6-21). Cuando se considera sólo la función $\mathrm{F}_{1}$ (Figura 6-16) y cuando $\mathrm{F}_{1}$ y $\mathrm{F}_{6}$ tienen más peso que las otras funciones (Figura 6-23), se 
obtienen los valores intermedios más altos. Vale la pena mencionar que el comportamiento de la variable PV estuvo influenciado por la cantidad de combustible fósil que se sustituirá en la minería.

La variable EOL obtuvo sus valores más altos al considerar la priorización única de las funciones $\mathrm{F}_{2}, \mathrm{~F}_{3}$ y $\mathrm{F}_{6}$ (Figuras 6-17 y 6-18). Cuando sólo se da prioridad a la función $\mathrm{F}_{6}$, esta variable obtuvo el tercer lugar en la priorización de alternativas, después de $\mathrm{CV}$ (Figura 6-21).

BL casi ha alcanzado su máximo potencial en la mayoría de los escenarios. Al igual que PV, el límite inferior de BL fue fijado. En este caso, a fin de satisfacer la demanda en el transporte.

La variable $\mathrm{MH}$ alcanza sus mejores valores cuando cada una de las funciones ambientales fueron priorizadas sobre las otras funciones, como 5 es a 1 . Asimismo, esta variable obtuvo su mayor valor (3.725 ktep) con $\mathrm{F}_{4}$ y F 5 (Figura 6-19).

Cuando se analiza el escenario "1,5,5,5,1,1” (Figura 6-22), las variables que se potenciaron significativamente fueron ST, LEÑ y CV; las variables EOL y MH también experimentaron aumento, pero en menor medida. En este escenario también se observa que la variable PV disminuye.

Con respecto al escenario " $5,1,1,1,1,5$ " (Figura 6-23), las variables que se potenciaron significativamente fueron $\mathrm{ST}$, PV, LEÑ y CV; la variable BL también experimentaron aumento, pero en menor medida. En este escenario también se observa que la variable EOL disminuye.

Resulta importante destacar que, cuando la función que maximiza el suministro sostenible de energía en las CRA $\left(\mathrm{F}_{6}\right)$ es considerada como única prioridad (Figura 6-21), la preferencia para la generación eléctrica se obtuvo para PV.

Vale la pena mencionar que los coeficientes de las funciones $F_{1}$ y $F_{6}$ han sido obtenidos sobre la base de una valoración cuantitativa de técnicos y expertos, por lo que la fiabilidad de estas funciones dependerá de su know-how sobre el área de estudio. Las otras funciones utilizan coeficientes basados en datos numéricos obtenidos de fuentes gubernamentales u otras fuentes oficiales. Esto puede convertirse en una limitación si la información es escasa. De acuerdo con esto, hay que destacar que el modelo no tiene la intención de indicar la "mejor" solución sobre las posibles alternativas, sino la de ayudar 
a contextualizar los pros y los contras relevantes al apoyar la toma de decisiones (Nerini et al. 2014).

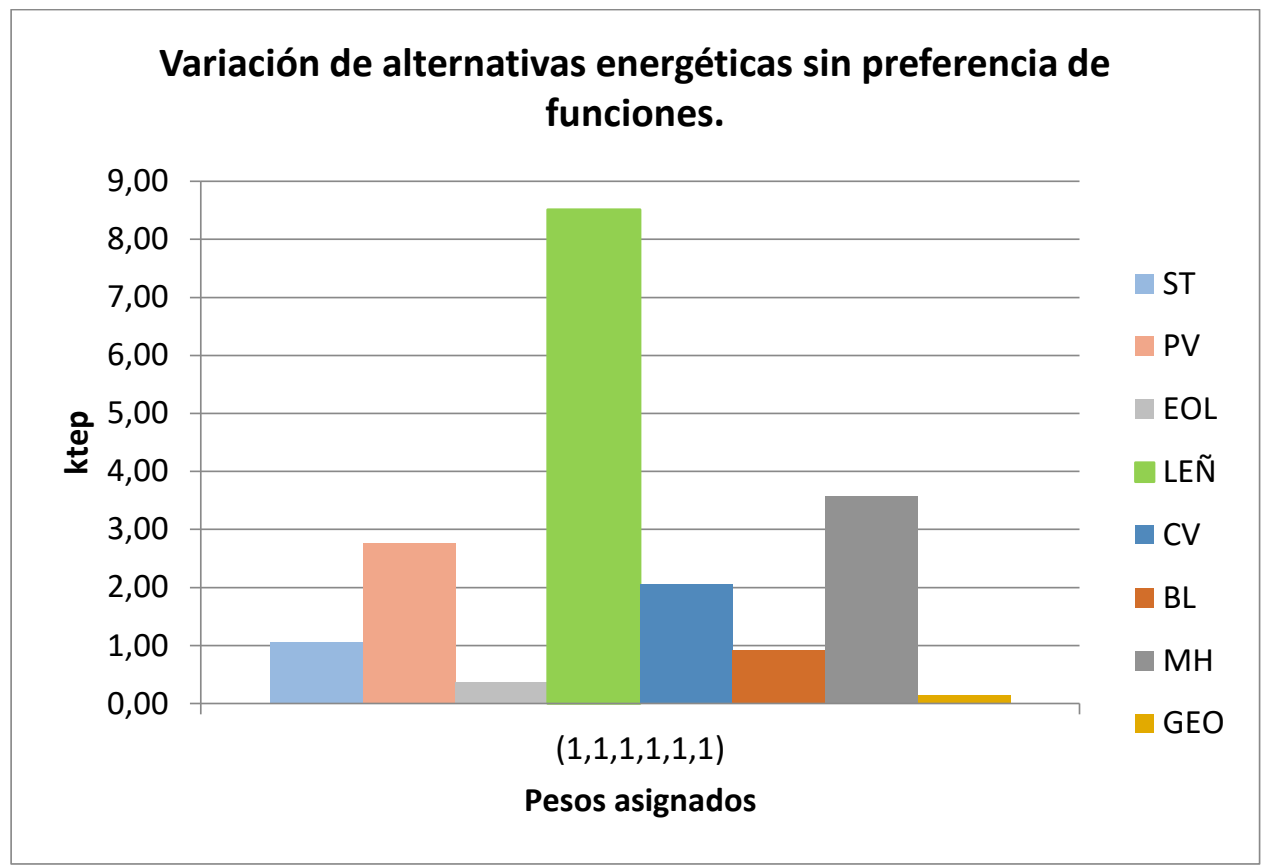

Figura 6-15. Resultado de la optimización no ponderada para cada alternativa energética. Distancia Chebyshev $\left(\mathrm{L}_{\infty}\right)$.

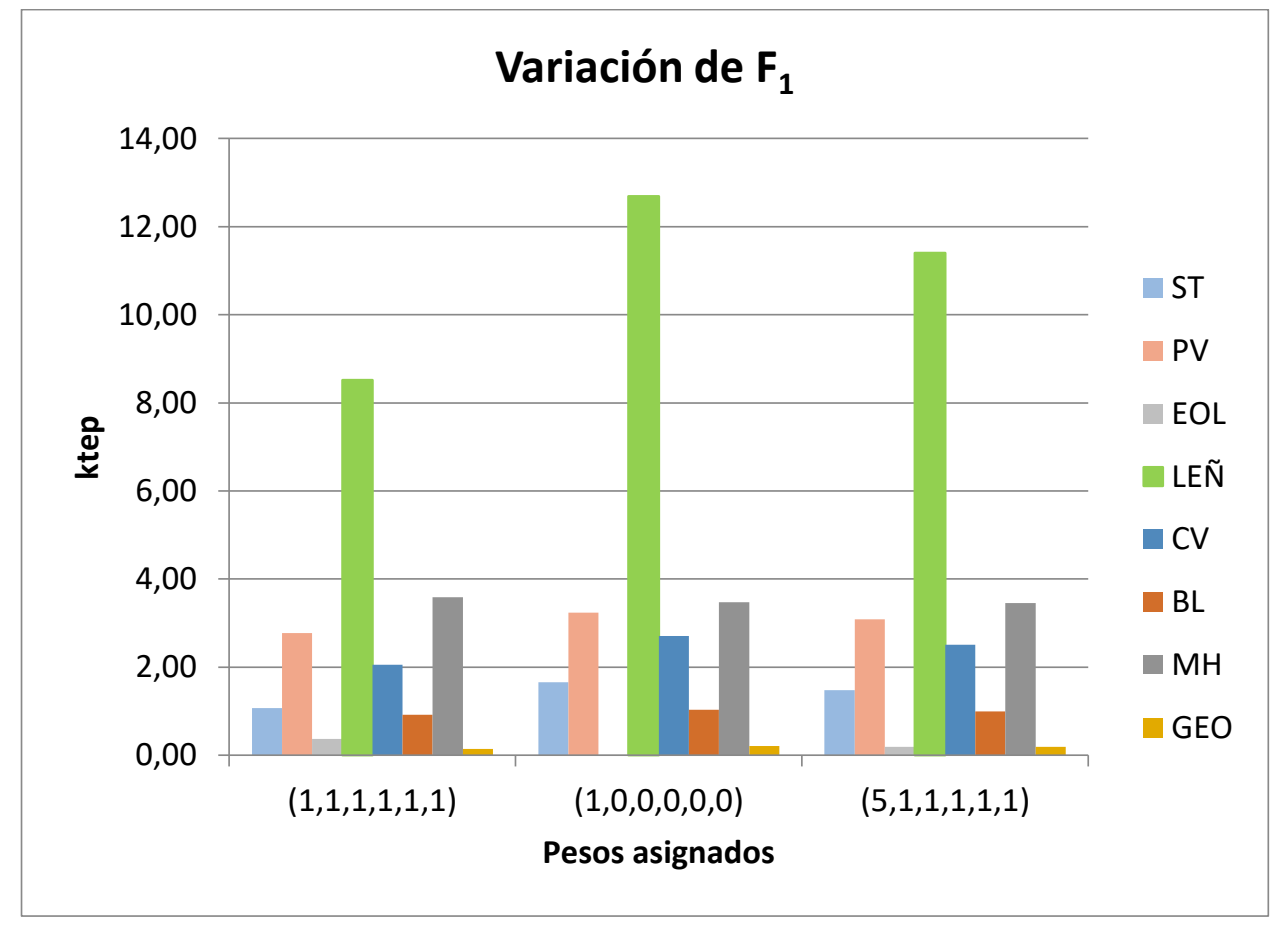

Figura 6-16. Resultados de la priorización de $\mathrm{F}_{1}$ para cada alternativa energética. Distancia Chebyshev $\left(\mathrm{L}_{\infty}\right)$. 


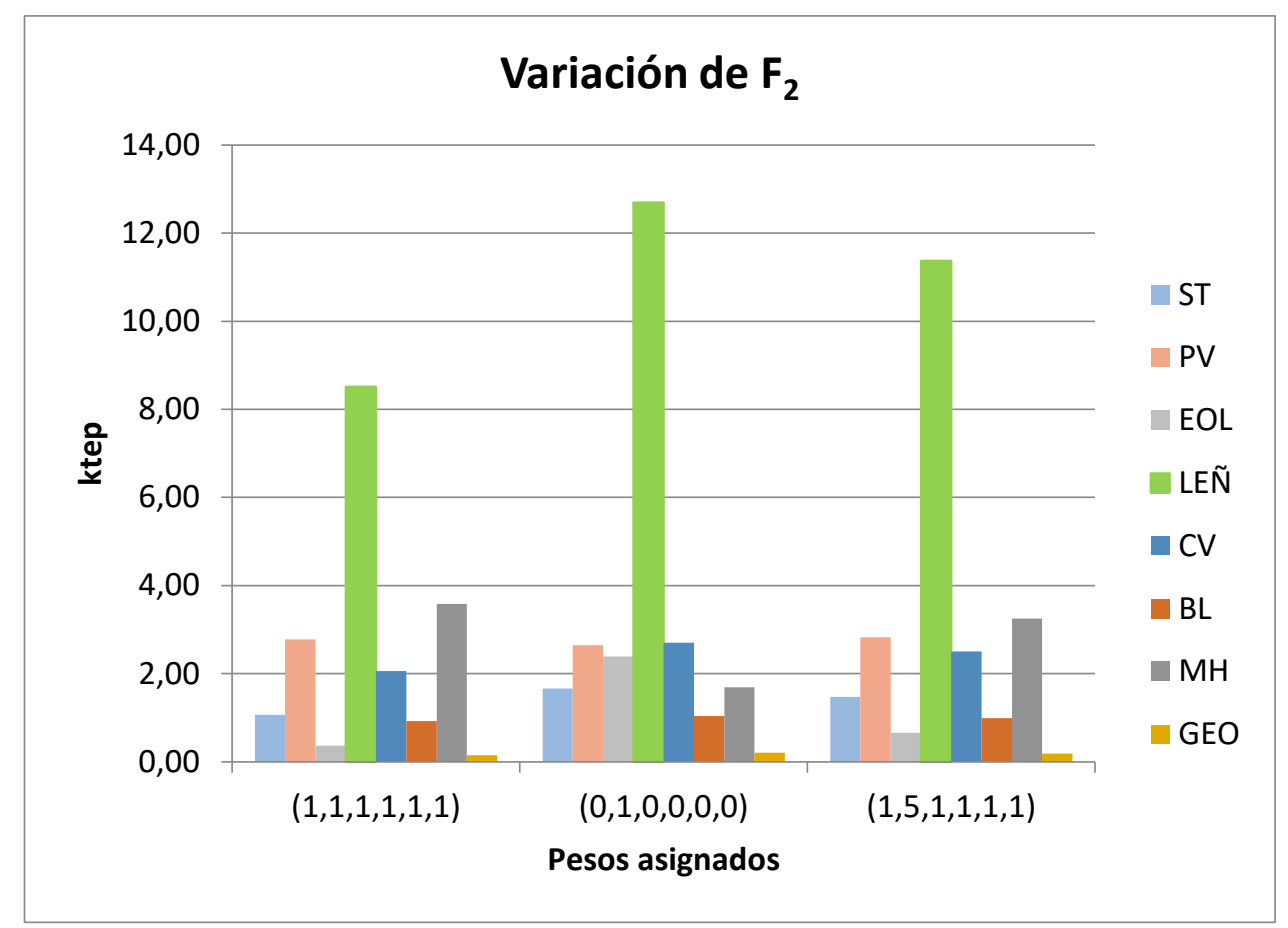

Figura 6-17. Resultados de la priorización de $F_{2}$ para cada alternativa energética. Distancia Chebyshev $\left(\mathrm{L}_{\infty}\right)$.

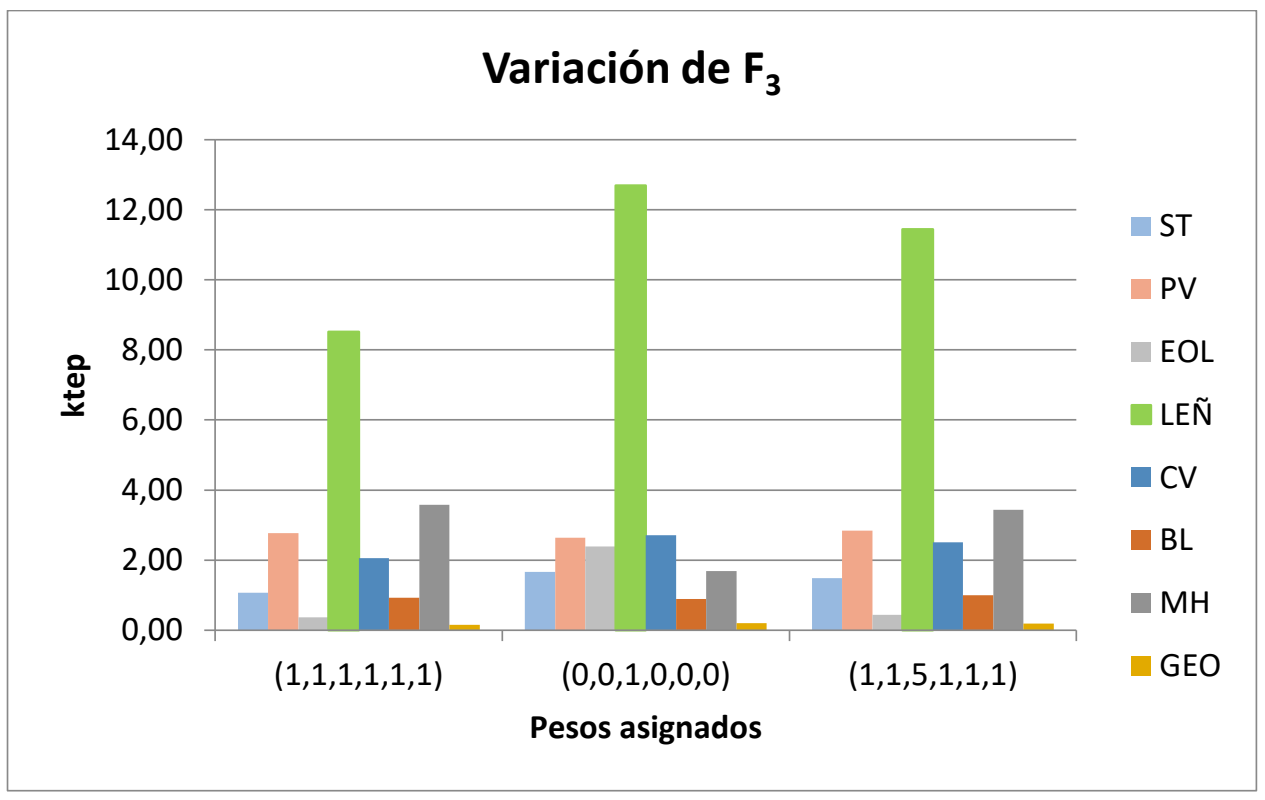

Figura 6-18. Resultados de la priorización de $\mathrm{F}_{3}$ para cada alternativa energética. Distancia Chebyshev $\left(\mathrm{L}_{\infty}\right)$. 


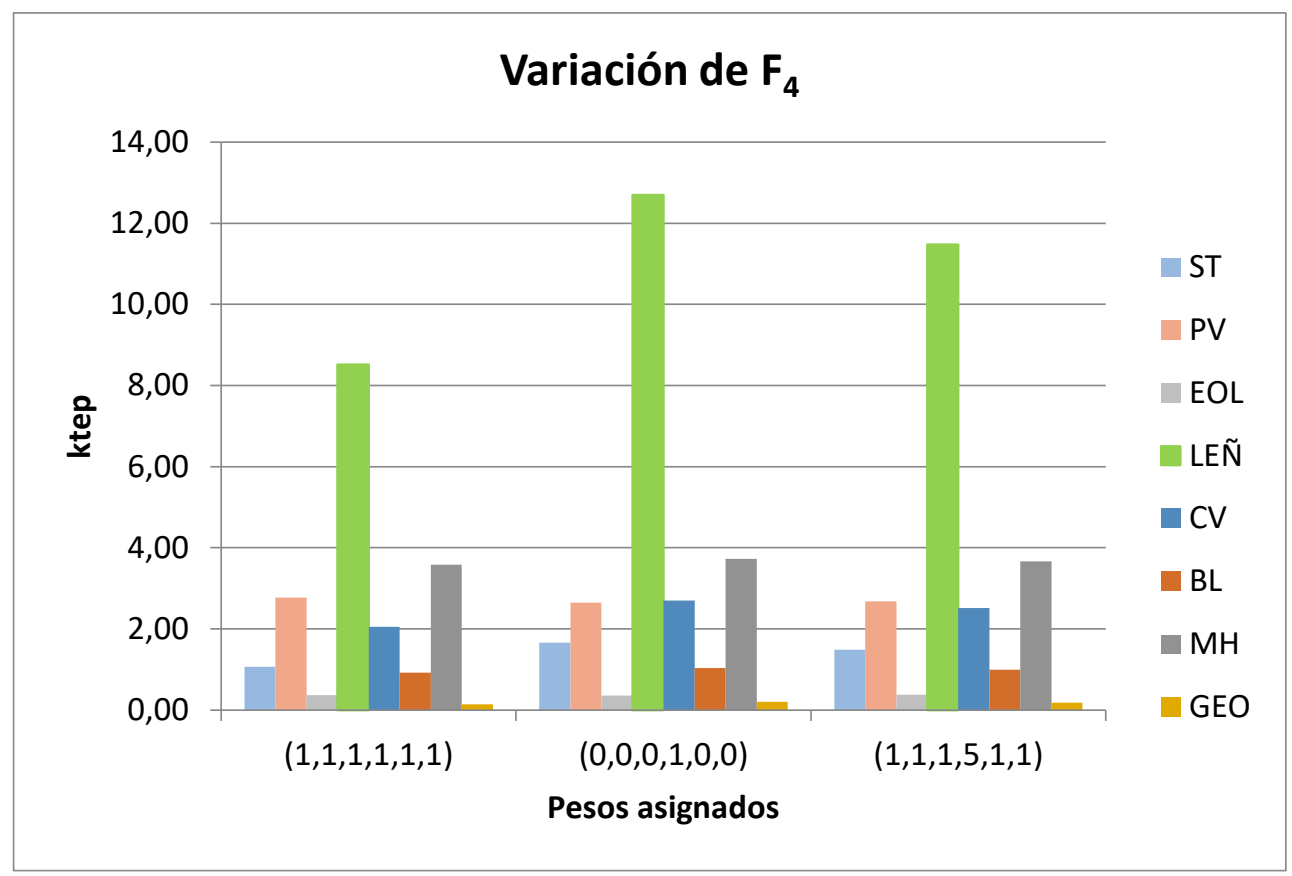

Figura 6-19. Resultados de la priorización de $\mathrm{F}_{4}$ para cada alternativa energética. Distancia Chebyshev $\left(\mathrm{L}_{\infty}\right)$.

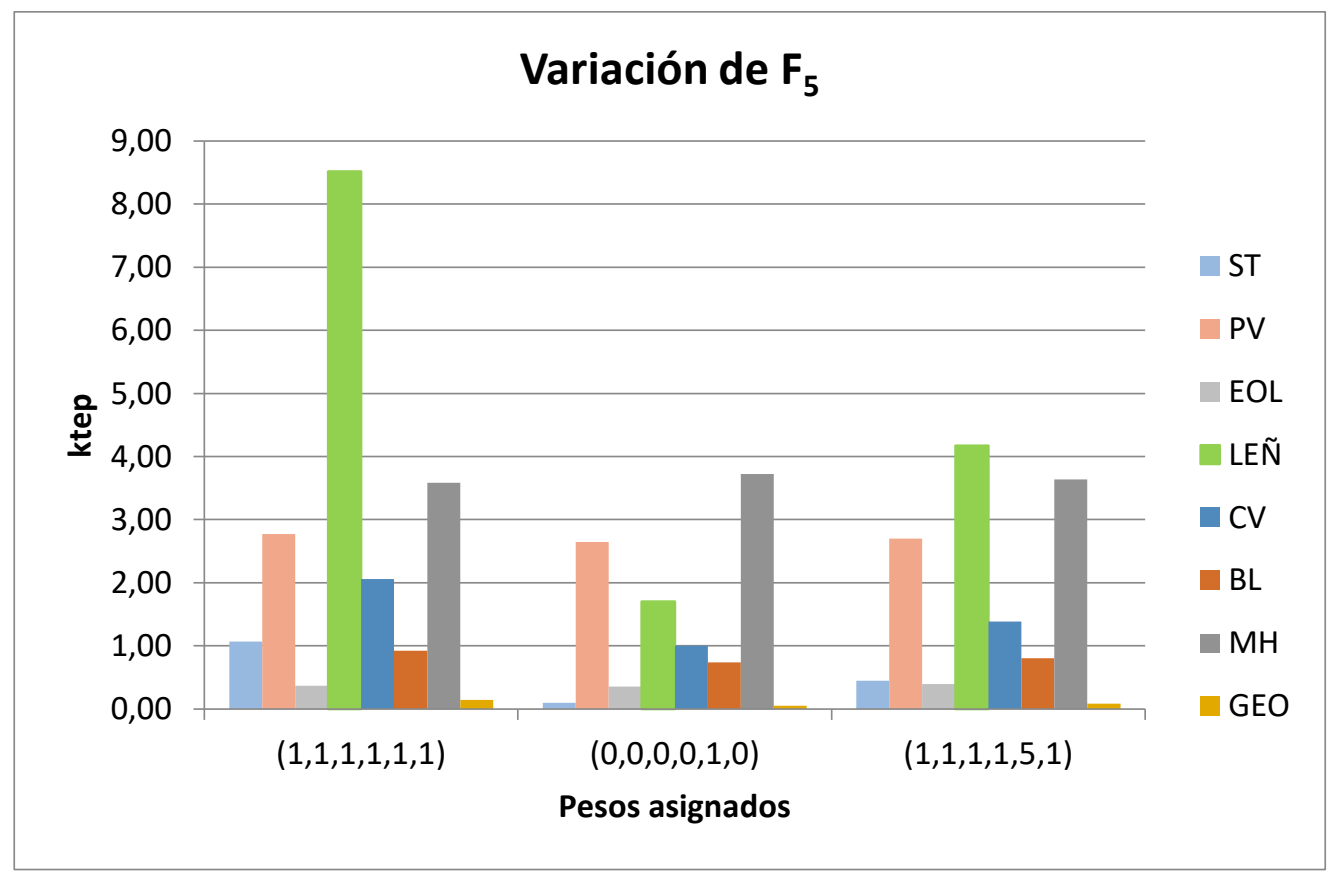

Figura 6-20. Resultados de la priorización de $\mathrm{F}_{5}$ para cada alternativa energética. Distancia Chebyshev $\left(\mathrm{L}_{\infty}\right)$. 


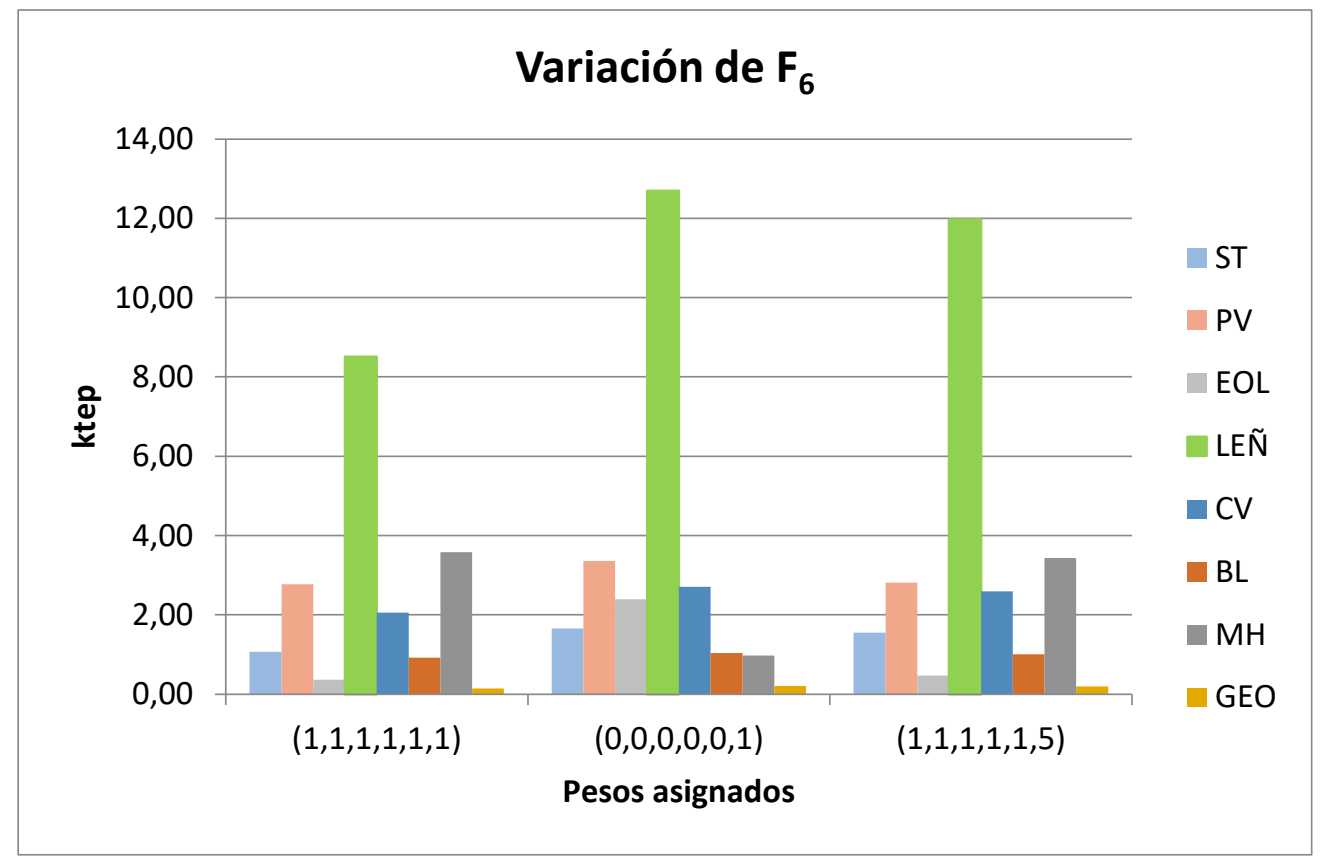

Figura 6-21. Resultados de la priorización de $\mathrm{F}_{6}$ para cada alternativa energética. Distancia Chebyshev $\left(\mathrm{L}_{\infty}\right)$.

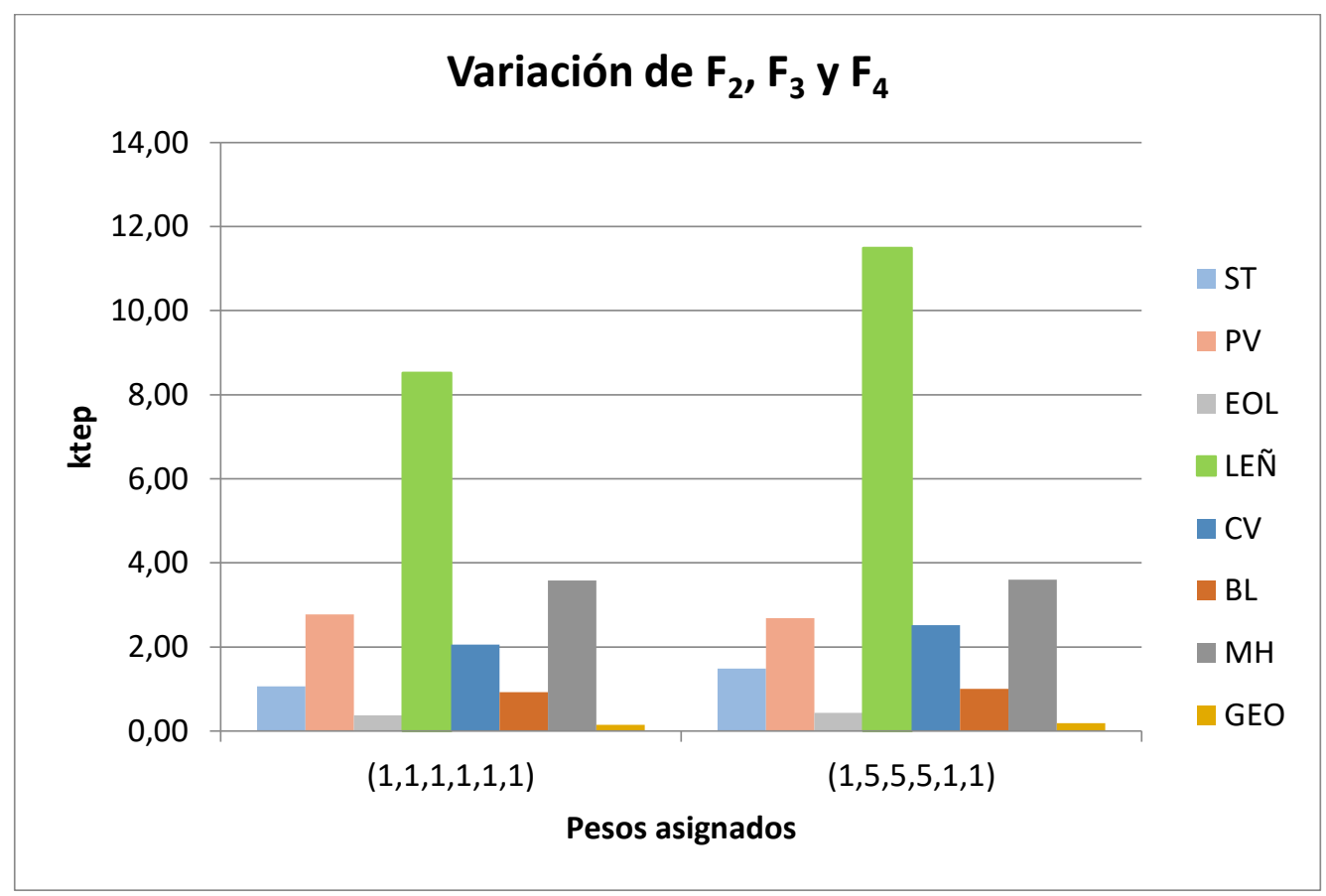

Figura 6-22. Resultados de la priorización de $\mathrm{F}_{2}, \mathrm{~F}_{3}$ y $\mathrm{F}_{4}$ para cada alternativa energética. Distancia Chebyshev $\left(\mathrm{L}_{\infty}\right)$. 


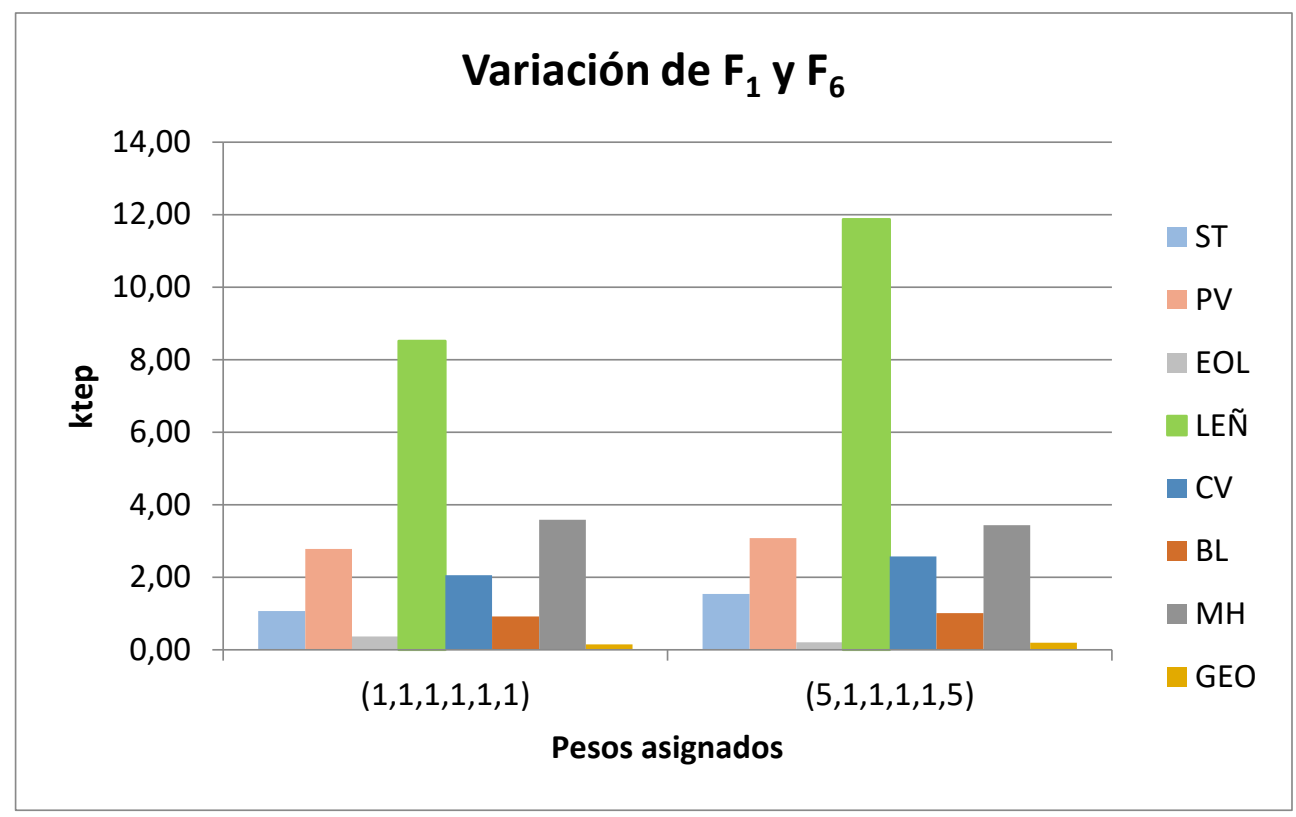

Figura 6-23. Resultados de la priorización de $\mathrm{F}_{1}$ y $\mathrm{F}_{6}$ para cada alternativa energética. Distancia Chebyshev $\left(\mathrm{L}_{\infty}\right)$.

Según los resultados presentados en este apartado, en la Tabla 6-2 se muestra un resumen con la priorización de las funciones, las cuales se muestran de mayor a menor importancia según los valores obtenidos para cada una de las alternativas energéticas consideradas. La distribución de pesos analizada es de 5 a 1 para cada función.

Tabla 6-3. Priorización de funciones según los máximos valores alcanzados por las alternativas energéticas renovables.

\begin{tabular}{cc}
\hline Energía renovable & $\begin{array}{c}\text { Priorización de funciones según } \\
\text { máximos valores alcanzados }\end{array}$ \\
\hline ST & $\mathrm{F}_{6}>\mathrm{F}_{4}>\mathrm{F}_{3}>\mathrm{F}_{1}>\mathrm{F}_{2}>\mathrm{F}_{5}$ \\
$\mathrm{PV}$ & $\mathrm{F}_{1}>\mathrm{F}_{3}>\mathrm{F}_{2}>\mathrm{F}_{6}>\mathrm{F}_{5}>\mathrm{F}_{4}$ \\
EOL & $\mathrm{F}_{2}>\mathrm{F}_{6}>\mathrm{F}_{3}>\mathrm{F}_{5}>\mathrm{F}_{4}>\mathrm{F}_{1}$ \\
LEN & $\mathrm{F}_{6}>\mathrm{F}_{4}>\mathrm{F}_{3}>\mathrm{F}_{1}>\mathrm{F}_{2}>\mathrm{F}_{5}$ \\
$\mathrm{CV}$ & $\mathrm{F}_{6}>\mathrm{F}_{4}>\mathrm{F}_{3}>\mathrm{F}_{1}>\mathrm{F}_{2}>\mathrm{F}_{5}$ \\
$\mathrm{BL}$ & $\mathrm{F}_{6}>\mathrm{F}_{4}>\mathrm{F}_{1}>\mathrm{F}_{3}>\mathrm{F}_{2}>\mathrm{F}_{5}$ \\
$\mathrm{MH}$ & $\mathrm{F}_{4}>\mathrm{F}_{5}>\mathrm{F}_{1}>\mathrm{F}_{3}>\mathrm{F}_{6}>\mathrm{F}_{2}$ \\
$\mathrm{GEO}$ & $\mathrm{F}_{6}>\mathrm{F}_{4}>\mathrm{F}_{3}>\mathrm{F}_{1}>\mathrm{F}_{2}>\mathrm{F}_{5}$ \\
\hline
\end{tabular}




\subsection{Análisis de sensibilidad de los resultados.}

Todo modelo de planificación energética que se pretenda aplicar en una zona específica se encontrará afectada por factores externos que pueden alterar los resultados esperados. La incidencia de los mismos estará relacionada a la naturaleza de las funciones objetivo que han sido consideradas en la formulación del modelo. Para el caso del modelo propuesto, algunos de los factores que podrían tener mayor impacto en los coeficientes de las funciones objetivo podrían ser: Cambios en la política energética, inestabilidad financiera, crisis política, desarrollo de tecnologías renovables con menores emisiones en su producción (mejor balance emisiones de análisis de ciclo de vida) y el precio del petróleo.

Es interesante destacar que las funciones obtenidas por el método de las precedencias (Funciones $\mathrm{F}_{1} \mathrm{y}_{\mathrm{F}}$ ) son capaces de introducir en su formulación dichos factores, ya que recogen la experiencia y punto de vista de expertos, los cuales se encuentran inmersos en la realidad local que se estudia. Esta característica representa una ventaja importante ya que el impacto de los factores mencionados sobre los coeficientes de las funciones objetivo podría ser difícil de predecir. Tal es el caso del desarrollo de tecnologías renovables con menores emisiones en su producción, cuyo impacto se daría principalmente en las funciones ambientales $\left(\mathrm{F}_{2}\right.$, $\mathrm{F}_{3}$ y $\mathrm{F}_{4}$ ). Un escenario distinto podría darse en el caso de la fluctuación del precio del petróleo, cuyo análisis está bastante extendido y existen diversos estudios sobre su impacto en el coste de las tecnologías energéticas. Por este motivo, en la presente tesis doctoral se ha desarrollado el análisis de sensibilidad de los resultados teniendo en cuenta dicho factor.

Sobre el impacto de las fluctuaciones del precio del petróleo en el coste de las tecnologías renovables, en el informe de la Agencia Internacional de Energías Renovables (IRENA) sobre los costes de generación energética con renovables se indica: "El desarrollo e implementación de las energías renovables representa la cobertura más segura a largo plazo contra la volatilidad del precio del combustible", asimismo, que "la caída de los precios del petróleo a fines de 2014 no altera sustancialmente la competitividad emergente de las energías renovables". En el caso concreto de la fotovoltaica, Branker et al. (2011) afirma que dicha tecnología no tiene un costo de combustible que sea susceptible al riesgo de fluctuación de 
precios. Por ello, se podría predecir que el impacto del factor en cuestión sobre las renovables no será significativo. Por otro lado, en el informe de consultoría del LCOE de Lazard de noviembre de 2016 (Lazard, 2016), en el análisis de sensibilidad de la fluctuación del precio de los combustibles fósiles sobre las energías renovables, sólo considera un impacto en el LCOE de la biomasa.

Teniendo en cuenta lo mencionado, y que la biomasa está vinculada al proceso de obtención de biocombustibles, en el análisis de sensibilidad se ha considerado el impacto de la fluctuación del precio del petróleo en los siguientes vectores energéticos: Leñas (LEÑ), Carbón Vegetal (CV), y Biocombustibles Líquidos (BL). Todos ellos de la función de costes $\left(\mathrm{F}_{5}\right)$, cuyos coeficientes están directamente vinculados al coste de las tecnologías energéticas. En las Figuras 624, 6-25 y 6-26 se presentan los resultados obtenidos para las alternativas energéticas mencionadas para un incremento del precio del petróleo en un $50 \%$, el cual representa una estimación del promedio de las mayores fluctuaciones del precio del crudo desde el 2009. En la Figura 6-27 se presenta el impacto en todas las alternativas energéticas renovables consideradas para el escenario sin preferencia de funciones $(1,1,1,1,1,1)$.

\section{Análisis de sensibilidad (AdS) para LEÑ con incremento de precio de petróleo en $50 \%$ para los distintos escenarios (Distancia $L_{\infty}$ )}

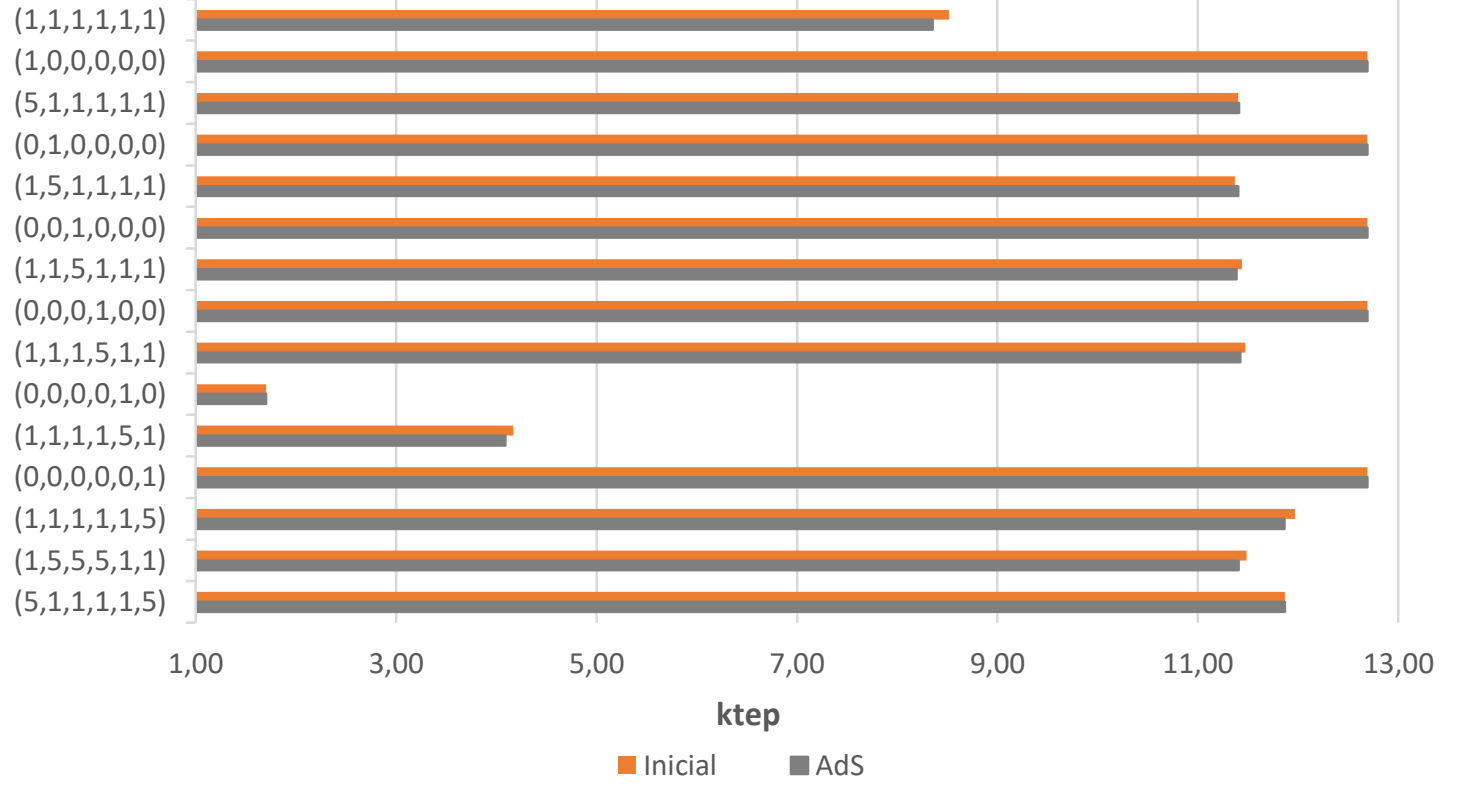

Figura 6-24. Resultados del análisis de sensibilidad para LEÑ con incremento del precio de petróleo en $50 \%$ para los distintos escenarios. (Distancia $\mathrm{L}_{\infty}$ ). 


\section{AdS para CV con incremento de precio de petróleo en $50 \%$ para los distintos escenarios (Distancia Lo)}

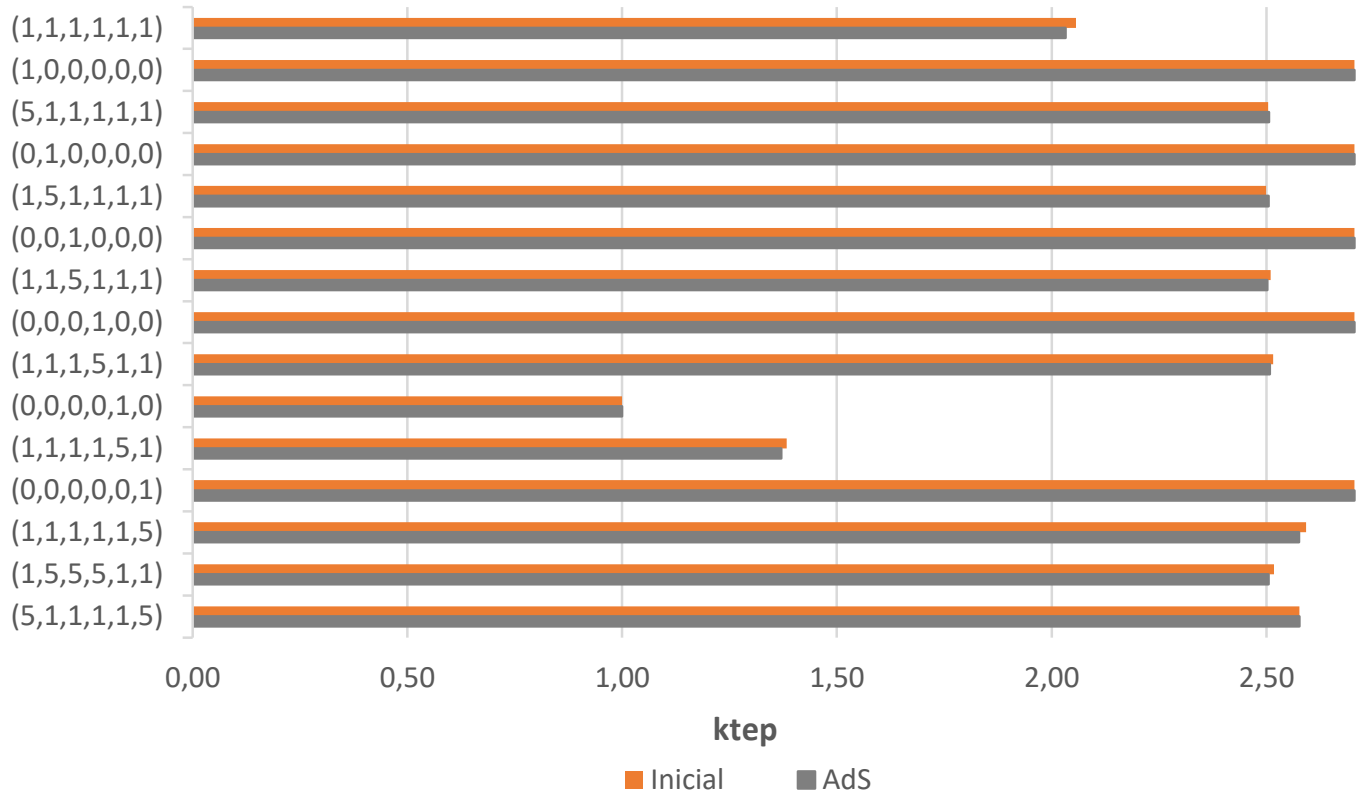

Figura 6-25. Resultados del análisis de sensibilidad para CV con incremento del precio de petróleo en $50 \%$ para los distintos escenarios. (Distancia $\mathrm{L}_{\infty}$ ).

\section{AdS para BL con incremento de precio de petróleo en $50 \%$ para los distintos escenarios (Distancia Lo)}

$(1,1,1,1,1,1)$

$(1,0,0,0,0,0)$

$(5,1,1,1,1,1)$

$(0,1,0,0,0,0)$

$(1,5,1,1,1,1)$

$(0,0,1,0,0,0)$

$(1,1,5,1,1,1)$

$(0,0,0,1,0,0)$

$(1,1,1,5,1,1)$

$(0,0,0,0,1,0)$

$(1,1,1,1,5,1)$

$(0,0,0,0,0,1)$

$(1,1,1,1,1,5)$

$(1,5,5,5,1,1)$

$(5,1,1,1,1,5)$

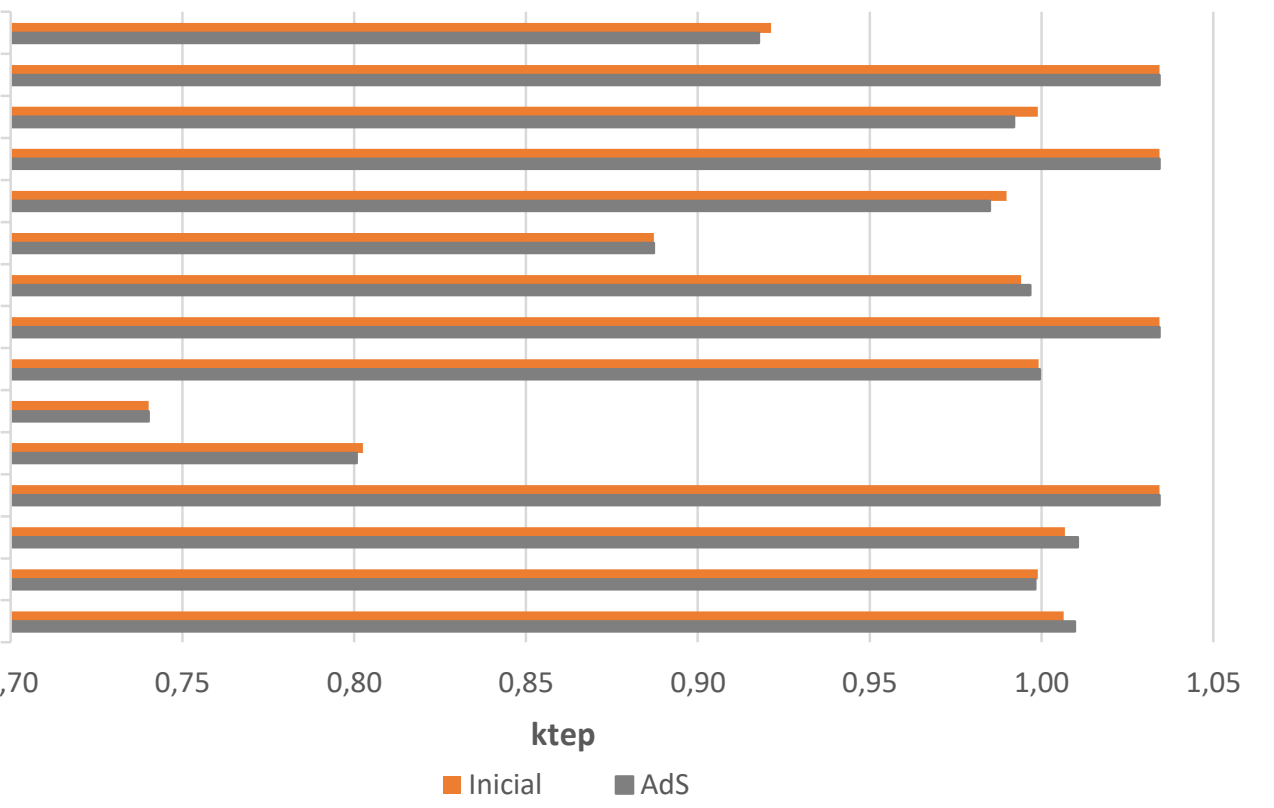

Figura 6-26. Resultados del análisis de sensibilidad para BL con incremento del precio de petróleo en $50 \%$ para los distintos escenarios. (Distancia $\mathrm{L}_{\infty}$ ). 


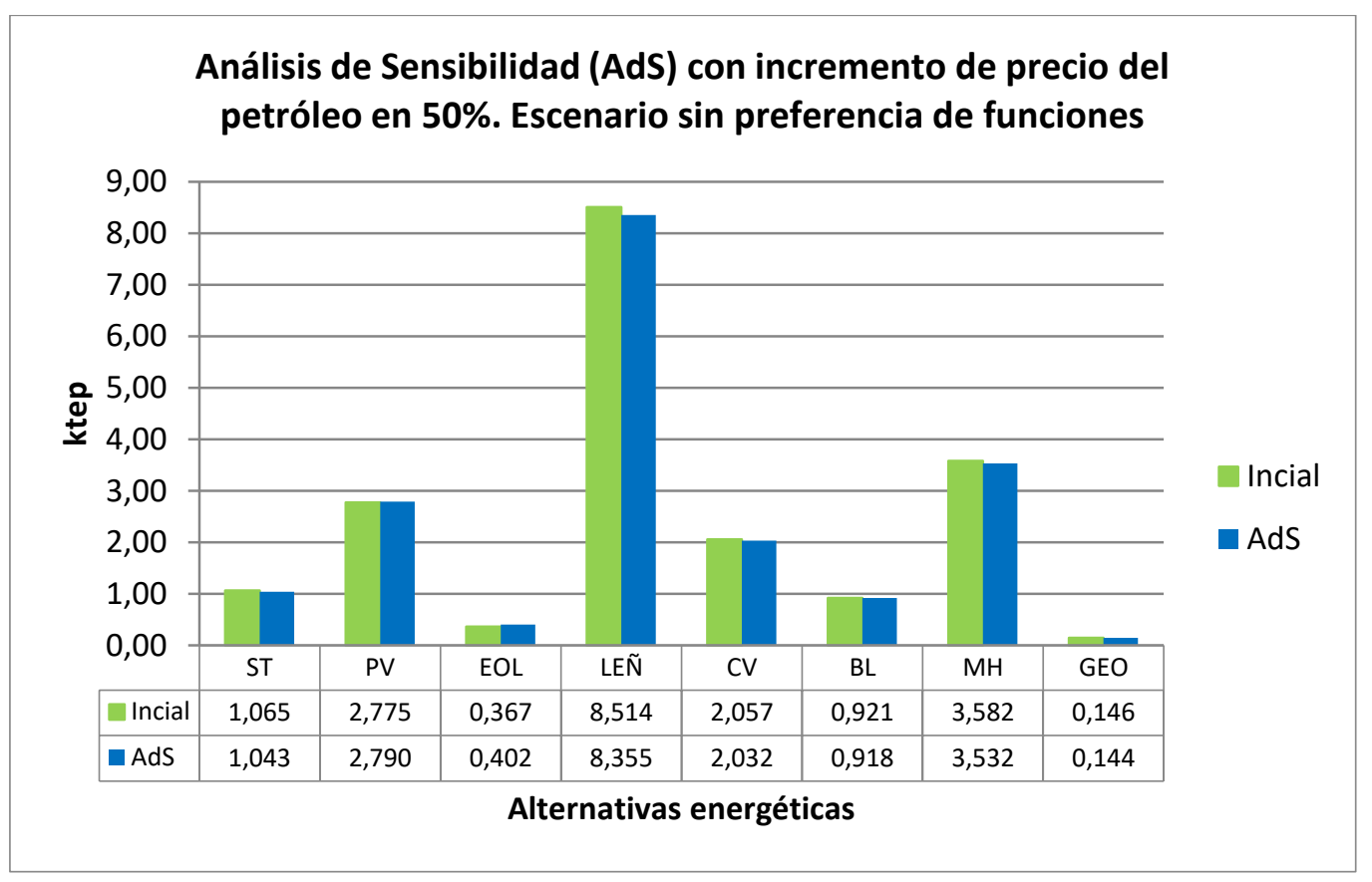

Figura 6-27. Resultados del análisis de sensibilidad con incremento del precio de petróleo en 50\% para las alternativas energéticas consideradas sin priorización de funciones.

En general se observa una variación muy pequeña con respecto a los resultados iniciales, asimismo, existe una tendencia a la disminución de dichas variables, lo cual es especialmente acusado en los escenarios sin priorización de funciones $(1,1,1,1,1,1)$ y donde la función de costes tiene mayor importancia $(1,1,1,1,5,1)$, resultados que podrían considerarse como esperados.

Además de los factores externos mencionados anteriormente, existen algunos que podrían alterar la oferta y la demanda de las energías renovables, por ejemplo, los desastres naturales y una variación importante del Producto Bruto Interno (PBI), que fue el indicador económico utilizado para proyectar la demanda en la presente tesis doctoral. Con el fin de conocer el impacto en la variación de este indicador en los resultados, se ha reducido el valor considerado inicialmente $(6,5 \%)$ a $3,5 \%$, representando por consiguiente una condición de recesión económica para el país.

En la Figura 6-28 se muestran los resultados obtenidos para las alternativas energéticas consideradas y su comparativa con los resultados iniciales. 


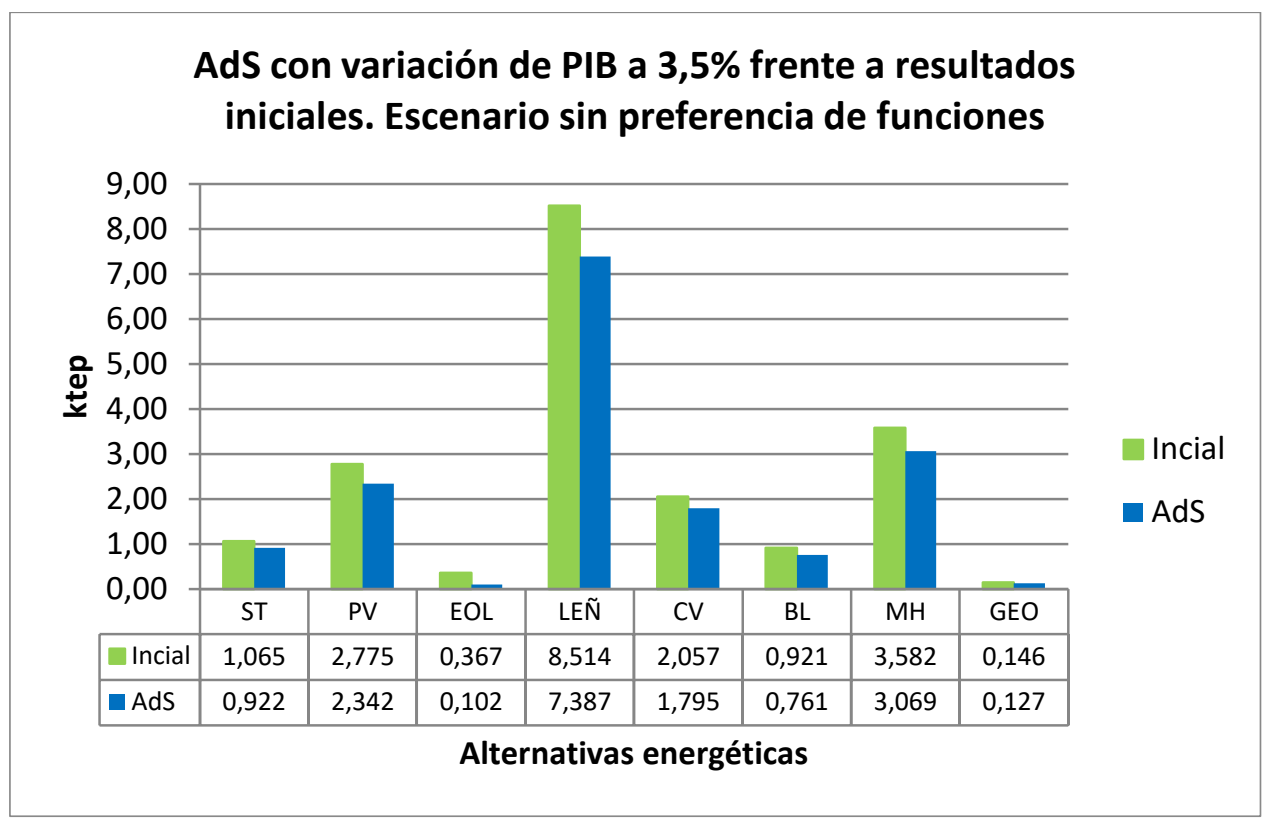

Figura 6-28. Resultados del análisis de sensibilidad con disminución del PBI a 3,5\% para las alternativas energéticas consideradas sin priorización de funciones.

Dado que la variación del PBI produjo la disminución de la demanda, las restricciones correspondientes también disminuyeron. Es también la tendencia que observa en los resultados de la Figura 6-28, donde todos los valores de las alternativas energéticas fueron inferiores a los obtenidos inicialmente. De este modo, los resultados confirman el comportamiento esperado de las variables.

\subsection{Comparativa del modelo SEPLAN, con los modelos PAMER y SEMA.}

En este apartado se ha realizado el análisis comparativo entre el modelo propuesto en la presente tesis doctoral y los modelos PAMER y SEMA, que han sido la base para el desarrollo del mismo.

Teniendo en cuenta que el modelo PAMER considera sólo la función de sustitución de energías fósiles por energías renovables $\left(\mathrm{F}_{1}\right)$ y el modelo SEMA, además de $\mathrm{F}_{1}$, considera la función de emisiones de $\mathrm{CO}_{2}\left(\mathrm{~F}_{2}\right)$ y la función de costes $\left(\mathrm{F}_{5}\right)$, ambos modelos han sido ejecutados con el programa Matlab®. Para ello, fueron consideradas las mismas restricciones del problema decisional. En la Figura 6-24 se muestran los resultados obtenidos. Con el fin de realizar una comparativa equitativa de los modelos, se ha considerado el escenario en el que todas las funciones objetivo tienen el mismo peso. 
La Figura 6-24 muestra, como era de esperarse, que la tendencia general de los resultados del modelo PAMER es maximizar las alternativas energéticas. Es interesante observar que en el caso de las variables $\mathrm{MH}$ y EOL, no se obtienen los valores máximos debido a la restricción de cumplir con la demanda de energía eléctrica planificada. Por otra parte, en el modelo SEMA, en la que se incorpora la función de emisiones de $\mathrm{CO}_{2}$ y la función de costes, se observa que una disminución de las variables, lo cual se considera lógico al introducir nuevos criterios en la planificación. Finalmente, se observa en el modelo SEPLAN que la mayoría de variables experimentan un ligero incremento, con excepción de PV y MH. En el caso de PV, su leve descenso se debió a la incorporación de dos nuevas funciones ambientales $\left(\mathrm{F}_{3}\right.$ y $\left.\mathrm{F}_{4}\right)$, las cuales han influido de manera más acusada que la función relacionada al acceso universal a la energía $\left(\mathrm{F}_{6}\right)$, donde ocupa un lugar prioritario. En el caso de MH, ocurrió la situación inversa, fue poco favorecida con la función $\mathrm{F}_{6}$, lo cual provocó su ligera diminución a pesar de obtener resultados mejores con las funciones ambientales $\mathrm{F}_{3}$ y $\mathrm{F}_{4}$.

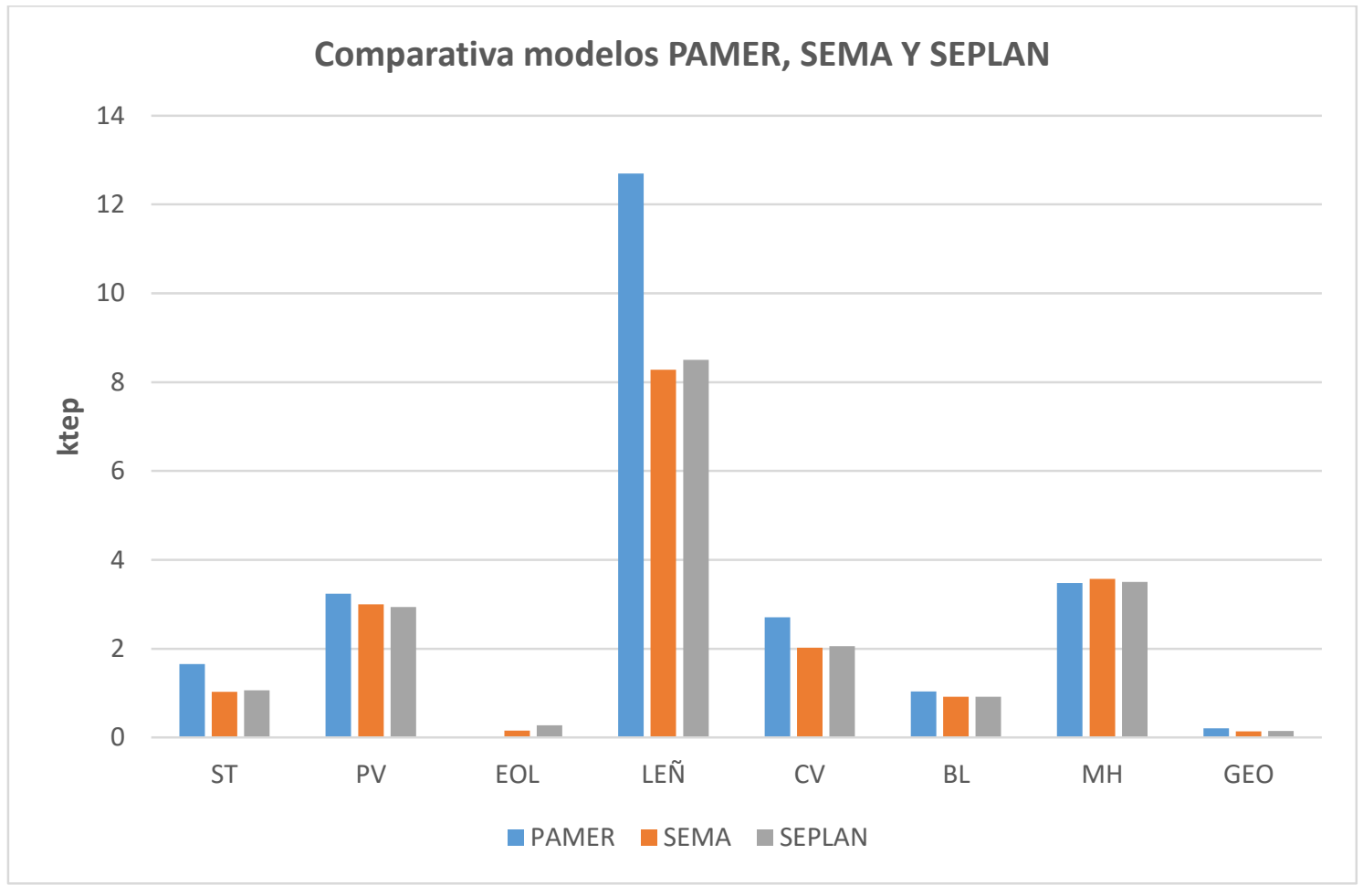

Figura 6-29. Comparativa de los modelos PAMER, SEMA y SEPLAN para las alternativas energéticas consideradas. 


\section{CAPÍTULO VII}

\section{CONCLUSIONES}

7.1 El modelo SEPLAN ha permitido considerar criterios cualitativos y cuantitativos en la planificación energética de la provincia de Cajamarca utilizando las técnicas de optimización multicriterio. De este modo, el suministro sostenible de energía en las comunidades rurales aisladas se ha incorporado en el problema decisional, así como, criterios económicos y ambientales. Asimismo, se han establecido varios escenarios, considerando diferentes opiniones de los centros decisores.

7.2 El estudio del potencial de aprovechamiento de las energías renovables en la provincia de Cajamarca permitió conocer la disponibilidad de dichos recursos y, de este modo, establecer las restricciones del modelo de planificación. La fuente renovable con mayor potencial aprovechable es sin duda la energía solar, sin embargo, el coste de la tecnología sigue siendo una limitante, por otro lado, la huella de carbono por kilovatio-hora de los sistemas fotovoltaicos continúa siendo superior al de los generadores eólicos o al de una mini central hidroeléctrica, lo cual ha penalizado su priorización en los escenarios estudiados. Sin embargo, se considera que, con apoyo gubernamental, a través de subvenciones que fomenten la inversión privada, podrá extenderse con mayor decisión el uso de la energía solar fotovoltaica, como se viene realizando actualmente para la electrificación de comunidades rurales aisladas.

7.3 Por los criterios asumidos en la función $\mathrm{F}_{6}$, que recoge la experiencia en electrificación rural de organizaciones localizadas en Perú, la aplicación del modelo SEPLAN podría ser más apropiada para países de Latinoamérica, sin embargo, dada las similitudes que podrían encontrarse sobre este tema en regiones de Asia o África, el modelo podría aplicarse también en estas zonas. En este sentido, el cálculo de los coeficientes de las funciones $\mathrm{F}_{1}$ y $\mathrm{F}_{6}$ será de especial relevancia para garantizar la fiabilidad del modelo de planificación. Por otro lado, en la región donde se pretenda aplicar el modelo, las funciones objetivo deberán ser consideradas relevantes por los centros decisores. 
7.4 La principal limitante del modelo propuesto corresponde a la información disponible. En el caso de las funciones medioambientales $\left(\mathrm{F}_{2}, \mathrm{~F}_{3}\right.$ y $\left.\mathrm{F}_{4}\right)$ y la de minimización de costes $\left(\mathrm{F}_{5}\right)$, cuyos coeficientes tuvieron valores cuantitativos, dichos valores fueron obtenidos de organismos nacionales e internacionales. La dificultad de obtener información local o regional, conlleva en muchos casos a extrapolar datos, con el riesgo de perder precisión en los resultados. En el caso de las funciones objetivo de maximización de energías renovables y de suministro sostenible de energía en comunidades rurales aisladas, los coeficientes se obtuvieron mediante la valoración de un comité de expertos, los cuales, teniendo en cuenta de una serie de criterios de comparación, valoraron cada alternativa energética; en este sentido, resulta importante consultar a profesionales con el know-how suficiente para obtener un resultado lo más realista posible.

7.5 Dentro de las características más importantes del modelo SEPLAN se destacan su facilidad para el modelamiento de escenarios y su potencia para la obtención de soluciones, cabe mencionar el aporte significativo del programa Matlab® para potenciarlas. Por otro lado, los escenarios estudiados brindan al centro decisor la posibilidad de conocer las diversas interacciones existentes entre las funciones objetivos y las alternativas energéticas estudiadas, lo cual representa un criterio clave para realizar una planificación energética adecuada.

7.6 Con respecto a la aplicación del modelo en la provincia de Cajamarca, las variables ST, LEÑ, CV y GEO fueron favorecidas en todos los escenarios, excepto en el caso en el que se minimiza la función de costes. Los valores alcanzados en cada caso corresponden a su máximo posible, estos son en ktep: $\mathrm{ST}=1,658$; $\mathrm{LEN}=12,693$; $\mathrm{CV}=2,705 ; \mathrm{GEO}=0,205$. Dentro de estas alternativas destacan la solar térmica (ST) y la geotérmica (GEO) por su alto potencial de expansión en la provincia, debido a su actual aprovechamiento y a su disponibilidad energética.

7.7 El comportamiento de los resultados obtenidos para las variables energéticas en los escenarios estudiados, manifiestan una clara diferencia entre las energías para uso térmico y eléctrico. En el primer caso, ST, LEÑ, CV, GEO y BL, tienen un comportamiento similar, en el caso de las energías para uso eléctrico: PV, EOL y 
$\mathrm{MH}$, tuvieron una tendencia distinta. Esto se debió principalmente a la restricción lineal de las energías de uso eléctrico que condicionaban su aplicación.

7.8 En el análisis de las variables energéticas, al priorizar la función de suministro sostenible de energía en comunidades rurales aisladas $\left(\mathrm{F}_{6}\right)$ como criterio único, la solar fotovoltaica (PV) obtuvo la prioridad para la generación eléctrica (2,87 ktep). Por otro lado, la solar térmica (SH) y la energía eólica (EOL) fueron favorecidas al priorizar las funciones de minimización del impacto ambiental.

7.9 En el análisis de sensibilidad realizado considerando un incremento del precio del petróleo y una disminución del Producto Bruto Interno (PBI), el comportamiento de los resultados fue el esperado, lo cual es un indicador de la robustez del modelo propuesto.

7.10 La comparación del modelo SEPLAN con los modelos PAMER y SEMA (Figura 6-24) comprueba que el aumento de criterios en el modelo de planificación resulta en el uso más equilibrado de los recursos energéticos, limitando su oferta según dichos criterios. Según esto, se podría deducir también que el modelo responde coherentemente al problema planteado.

7.11 En cuanto a futuros trabajos, el modelo propuesto podría incorporar otras funciones objetivo que sean relevantes para la región a estudiar. Asimismo, se pueden analizar otros tipos de emisiones. Podría ser de interés tener en cuenta los sistemas híbridos en una función objetivo, ya que estos sistemas son ampliamente utilizados en las comunidades rurales aisladas. 


\section{REFERENCIAS BIBLIOGRÁFICAS}

1. ACCIONA Microenergía Perú, 2011. Perfil del proyecto de electrificación de las localidades de la zona rural de Cachachi, La Encañada, Namora, San Pablo, Tumbaden e Ichocan, en la región Cajamarca, empleando energía solar fotovoltaica, Cajamarca: ACCIONA Microenergía Perú.

2. Agencia de Cooperación Internacional del Japón, 2012. Plan maestro para el desarrollo de la energía geotérmica en el Perú. [En línea] Disponible en: $\quad$ http://open jicareport.jica.go.jp/pdf/12048567.pdf [Último acceso: Febrero 2016].

3. Alario, J., 1981. Modelo MEDEE. Modelo de previsión a medio y largo plazo de la demanda de energía en Cataluña. Madrid, Simposio sobre modelos energéticos. Comisaría de la Energía y Recursos Minerales.

4. Álvarez, P., 2004. Modelo de planificación energética orientado al desarrollo sostenible. Madrid: Tesis doctoral. E.T.S.I. Minas. Universidad Politécnica de Madrid.

5. Amer, M. \& Daim, T. U., 2011. Selection of renewable energy technologies for a developing county: A case of Pakistan. Energy for Sustainable Development, Volumen 15, pp. 420-435.

6. Banco Central de Reserva del Perú, 2016. [En línea] Disponible en: http://www.bcrp.gob.pe/docs/Sucursales/Trujillo/cajamarcacaracterizacion.pdf.

[Último acceso: Noviembre 2017].

7. Batlle, C., Barroso, L. \& Echevarría, C., 2012. Evaluación del marco normativo e institucional del Perú para la promoción de energía eléctrica a partir de recursos renovables. BID - División de Energía INE/ENE. [En línea] Disponible en: http://www.iadb.org/wmsfiles/products/publications/documents/37357374.pdf [Último acceso: Abril 2016].

8. Belton, S. \& Stewart, T. S., 2002. Multiple Criteria Decision Analysis. An Integrated Approach. Massachusetts: Kluwer Academic Publishers.

9. Benitez, L., 2015. Procedimiento multicriterio-multiobjetivo de planificación energética a comunidades rurales aisladas. Madrid: Tesis doctoral. Universidad Politécnica de Madrid.

10. Binder, W., Paredis, C. \& Garcia, H., 2014. Hybrid Energy System Modeling in Modelica. Lund, Sweden, Proceedings of the 10th International Modelica Conference; March 10-12.

11. Branker, K., Pathak, M. \& Pearce, J., 2011. A review of solar photovoltaic levelized cost of electricity. Renewable and Sustainable Energy Reviews, Issue 15 , p. $4470-4482$. 
12. Bustinduy, A., 1983. El modelo ESMERALDA. Madrid, I Curso de Planificación Energética. Universidad Politécnica de Madrid.

13. Buyukkaya, E., 2010. Effects of biodiesel on a DI diesel engine performance, emission and combustion characteristics. Fuel, Issue 89, pp. 3099-3105.

14. Campos, S. \& Salazar, S., 2013. Diseño de una planta industrial de biodiesel a partir de la semilla de higuerilla en el valle de Condebamba - Cajamarca. Cajamarca: Trabajo fin de carrera. Facultad de Ingeniería Industrial. Universidad Privada del Norte.

15. Carton, C., 1996. Reforestación y Manejo de Cuencas en la CAT. Atahualpa Jerusalén Granja Porcón, Cajamarca. En: Curso - Taller Manejo Integral de Microcuencas Jequetepeque - Cajamarca (21-30 de octubre de 1996). Cajamarca: CONDESAN, CIP, ADEFOR.

16. Chateau, B. \& Lapillonne, B., 1977. La prévision á long tenue de la demande d'énergie, propositions méthodologiques. París: CNRS. Energie et Société.

17. Chateau, B. \& Lapillonne, B., 1979. The MEDEE models for long term energy demand forecasting. Energy Systems Analysis, Dublín International Conference, 9 - II october. Dordrecht, D. Reidel Publishing Company.

18. Cherni, J. A. y otros, 2007. Energy supply for sustainable rural livelihoods. A multi-criteria decision-support system. Energy Policy, Issue 35, p. 1493-1504.

19. CIEMAT, 2014. CIEMAT. [En línea] Disponible en: http://www.ciemat.es/ [Último acceso: Octubre 2016].

20. Consejo Nacional del Ambiente, 2005. Indicadores Ambientales de Cajamarca. Serie Indicadores Ambientales $N^{o} 6 . \quad$ [En línea] Disponible en: http://bibliotecavirtual.minam.gob.pe/biam/bitstream/id/583/BIV00133.pdf [Último acceso: Marzo 2016].

21. Consorcio de Investigación Económica y Social, 2016. Elecciones Perú 2016, centrando el debate electoral: Acceso Universal a la Energía y Tecnologías Renovables. línea] Disponible en: http://www.cies.org.pe/sites/default/files/investigaciones/accesouniversal-a-la-energia-y-tecnologias-renovables.pdf [Último acceso: Abril 2016].

22. Delta Volt, 2016. Energías Renovables. [En línea] Disponible en: http://deltavolt.pe/energia-renovable/renovable-peru [Último acceso: Abril 2017].

23. Departamento de Energía, EE.UU, 2002. Sistemas eólicos pequeños para generación de electricidad. [En línea] Disponible en: http://apps2.eere.energy.gov/wind/windexchange/pdfs/small_wind/small_wind 
guide spanish.pdf

[Último acceso: Febrero 2016].

24. Díaz, A., Moreno, A., Cabezudo, C. \& Castro, A., 2013. Elaboración de un índice de Sostenibilidad de las Cocinas Mejoradas en los departamentos de Tacna, Moquegua, Arequipa, Ayacucho, Huancavelica, Cajamarca y San Martin. Lima: EnDev/GIZ.

25. Domínguez, L. C., 2015. Sustainable and renewable implementation multicriteria energy model (SRIME) - Case Study: Sri Lanka. Madrid: Tesis doctoral. Universidad Europea de Madrid.

26. Domínguez, L. C. \& Marcos, F., 2015. Sustainable and renewable implementation multi-criteria energy model (SRIME) - Case study: Sri Lanka. International Journal of Energy and Environmental Engineering, 6(2), pp. 165-181.

27. Falcón-Roque, E. y otros, 2017. Energy planning model with renewable energy using optimization multicriteria techniques for isolated rural communities: Cajamarca province, Peru. Journal of Renewable and Sustainable Energy, 9(6).

28. FAO, 2010. Análisis de recursos biomásicos leñosos y de residuos para uso combustible. En: Bioenergía y seguridad alimentaria "BEFS". El análisis de BEFS para el Perú. Roma: FAO, pp. 71-95.

29. FAO, 2011. Estado del Arte y Novedades de la Bioenergía en el Perú. [En línea] Disponible en: http://www.fao.org/docrep/019/as415s/as415s.pdf

30. Ferrer-Martí, L., Domenech, B., García-Villoria, A. \& Pastor, R., 2013. A MILP model to design hybrid wind-photovoltaic isolated rural electrification projects in developing countries. European Journal of Operational Research, Volumen 226, p. 293-300.

31. Fondo Multilateral de Inversiones, 2013. Luz en Casa. Acceso a energía sostenible en comunidades rurales. [En línea] Disponible en: http://www.fomin.org/Portals/0/Topics/BSS_energia_accionaperu.pdf [Último acceso: Febrero 2016].

32. Fondo Multilateral de Inversiones, 2014. De las velas a la luz eléctrica. El impacto de la electrificación rural. [En línea] Disponible en: http://www.fomin.org/Portals/0/impact\%20evaluation/PanelesSolares(Peru).pdf. [Último acceso: Mayo 2016].

33. Francisco, 2015. Laudato Si. Carta Encíclica. [En línea] Disponible en: $\quad$ https://laudatosi.com/watch [Último acceso: Octubre 2017].

34. Freimer, M. \& Yu, P. L., 1976. Some new results on compromise solutions for group decision problems. Management Science, Issue 22, p. 688-693. 
35. Fundación ACCIONA Microenergía, 2015a. Modelo LC-SFD de ACCIONA Microenergía Perú. Informe de escalabilidad, Madrid: Centro de Innovación de Tecnologías para el Desarrollo Humano de la Universidad Politécnica de Madrid.

36. Fundación ACCIONA Microenergía, 2015b. Modelo LC-SFD de ACCIONA Microenergía Perú. Informe de replicabilidad, Madrid: Centro de Innovación de Tecnologías para el Desarrollo Humano de la Universidad Politécnica de Madrid.

37. García, L., 2004. Desarrollo de un modelo multicriterio-multiobjetivo de oferta de energías renovables: aplicación a la comunidad de Madrid. s.1.:Tesis doctoral. Universidad Politécnica de Madrid.

38. Gobierno Regional de Cajamarca, 2011. Estudio Hidrológico de la Región Cajamarca 2010-2011. [En línea] Disponible en: http://zeeot.regioncajamarca.gob.pe/sites/default/files/HIDROLOGIA.pdf [Último acceso: Febrero 2016].

39. Grupo de Apoyo al Sector Rural de la Pontificia Universidad Católica del Perú, 2017. El desarrollo de la energía solar en el Perú. Nota 01/03/2017. [En línea] Disponible en: http://gruporural.pucp.edu.pe/nota/el-desarrollo-de-la-energiasolar-en-el-peru/

[Último acceso: Septiembre 2017].

40. Hidrandina S.A., 2014. I Foro Regional de Energía de Cajamarca. [En línea] Disponible en: http://www.osinergmin.gob.pe/newweb/uploads/Publico/OficinaComunicacione s/EventosRealizados/ForoCajamarca/2/Atencion $\% 20 \mathrm{de} \% 20 \mathrm{demanda} \% 20 \mathrm{Cajam}$ arca.pdf

[Último acceso: Febrero 2016].

41. Holmes, J. C., 1972. An ordinal method of evaluation. Urban Studies, 9(2), pp. 179-191.

42. Huang, Z., Yu, H., Peng, Z. \& Zhao, M., 2015. Methods and tools for community energy planning: A review. Renewable and Sustainable Energy Reviews, Volumen 42, p. 1335-1348.

43. Indacochea, A. y otros, 1998. Cajamarca Competitiva. Lima: s.n.

44. Iniyan, S., Suganthi, L. \& Jagadeesan, T. R., 1998. Renewable Energy Planning for India in 21st century. Renewable Energy, 14(1-4), pp. 453-457.

45. Instituto Nacional de Estadística e Informática (INEI), 2008. Censos Nacionales 2007: XI de Población y VI de Vivienda. [En línea] Disponible en: https://www.inei.gob.pe/media/MenuRecursivo/publicaciones_digitales/Est/Lib1 136/libro.pdf.

[Último acceso: Febrero 2016].

46. Instituto Nacional de Estadística e Informática, 2010. Perú: Estimaciones y proyecciones de población departamental por años calendario y edades simples 
1995-2025.

$[$ En

línea]

Disponible

en:

http://proyectos.inei.gob.pe/web/biblioineipub/bancopub/Est/Lib1039/libro.pdf

[Último acceso: Febrero 2016].

47. Instituto Nacional de Estadística e Informática, 2013. Anuario de Estadísticas Ambientales 2013. Capitulo 7. Energía, Minería e Hidrocarburos. [En línea] Disponible en: https://www.inei.gob.pe/media/MenuRecursivo/publicaciones_digitales/Est/Lib1 140/cap07.pdf

[Último acceso: Marzo 2016].

48. Instituto Nacional de Estadística e Informática, 2015. Información Económica. [En línea]

Disponible en: http://iinei.inei.gob.pe/iinei/siemweb/publico/ [Último acceso: Enero 2016].

49. Instituto Peruano de Economía, 2015. Datos Cajamarca. [En línea] Disponible en: $\quad$ http://www.ipe.org.pe/cajamarca [Último acceso: Noviembre 2017].

50. International Energy Agency, 2012. World Energy Model 2012. [En línea] Disponible en: https://www.iea.org/publications/freepublications/publication/English.pdf [Último acceso: Noviembre 2016].

51. International Energy Agency, 2016. World Energy Outlook 2015, Paris: IEA.

52. International Energy Agency, 2016. World Energy Outlook 2016, Paris: IEA.

53. IRENA, 2014. Peru Renewables Readiness Assessment 2014. [En línea] Disponible en: http://www.irena.org/DocumentDownloads/Publications/RRA_Peru.pdf [Último acceso: Febrero 2016].

54. IRENA, 2015. Renewable Power Generation Costs in 2014. [En línea] Disponible en: http://www.irena.org/documentdownloads/publications/irena_re_power_costs_2 014 report.pdf [Último acceso: Mayo 2016].

55. IRENA, 2016. The Renewable Route to Sustainable Transport-A Working Paper based on REmap. [En línea] Disponible en: http://www.irena.org/DocumentDownloads/Publications/IRENA_REmap Trans port_working paper_2016.pdf [Último acceso: Septiembre 2016].

56. Janardan, K. \& Schaiffer, 1975. Illinois Water Quality Inventory Report. Chicago: U.S. Environmental Protection Agency. 
57. Jebaraj, S. \& Iniyan, S., 2006. A review of energy models. Renewable and Sustainable Energy Reviews, 10(4), pp. 281-311.

58. Jorgenson, D., 1998. Growth, Volume 1: Econometric General Equilibrium Modeling. Cambridge: MIT Press.

59. Kanase-Patil, A. B., Saini, R. P. \& Sharma, M. P., 2010. Integrated renewable energy systems for off grid rural electrification of remote area. Renewable Energy, Issue 35, p. 1342-1349.

60. Karjalainen, J., Kákstinen, M., Luukkanen, J. \& Vehmas, J., 2014. Energy models and scenarios in the era of climate change, Briefing Report. Turku: Finland Futures Research Centre.

61. Kaya, T. \& Kahraman, C., 2010. Multicriteria renewable energy planning using an integrated fuzzy VIKOR \& AHP methodology: The case of Istanbul. Energy, Issue 35, p. $2517-2527$.

62. Kaya, T. \& Kahraman, C., 2011. Multicriteria decision making in energy planning using a modified fuzzy TOPSIS methodology. Expert Systems with Applications, Issue 38, p. 6577-6585.

63. Kumar, A. y otros, 2017. A review of multi criteria decision making (MCDM) towards sustainable. Renewable and Sustainable Energy Reviews, Issue 69, p. 596-609.

64. Kydes, A. S. \& Rabinowitz, J., 1981. Overview and special features of the timestepped energy system optimization model (TESOM). Resources and Energy, 3(1), pp. 65-92.

65. Kydes, S., 1980. The Brookhaven Energy System Optimization Model: Its Variants and Uses. En: Z. W. T. \& S. S. L., edits. Energy Policy Modelling: United States and Canadian Experiences. Dordrecht: Springer, pp. 110-136.

66. Lambert, T., Gilman, P. \& Lilienthal, P., 2006. Micropower system modeling with HOMER. En: F. Farret \& M. Simoes, edits. Integration of alternative sources of energy. s.1.:John Wiley \& Sons, Inc., pp. 379-418.

67. Lazard, 2016. Lazard's Levelized Cost of Energy Analysis - Version 10.0. [En línea]

Disponible en: https://www.lazard.com/perspective/levelized-cost-of-energyanalysis-100/

[Último acceso: Octubre 2017].

68. Lillo, P., Ferrer-Martí, L., Boni, A. \& Fernández-Baldor, A., 2015. Assessing management models for off-grid renewable energy electrification projects using the Human Development approach: Case study in Peru. Energy for Sustainable Development, Issue 25, p. 17-26.

69. Løken, E., 2007. Use of multicriteria decision analysis methods for energy planning problems. Renewable and Sustainable Energy Reviews, Volumen 11, p. $1584-1595$. 
70. López, F., 2014. Potencial hidroenergético en la zona norte del Perú, San Martin: Dirección General de Energía y Minas.

71. Loulou, R. \& Labriet, M., 2008. ETSAP-TIAM: the TIMES integrated assessment model Part I: Model structure. $C M S$, Issue 5, p. 7-40.

72. Marcos, F., 1985. Aplicación de las técnicas multidimensionales a la planificación energética. Energía, pp. 97-104.

73. Marglin, J. A., 1967. Public Investment Criteria. Cambridge. Massachusetts: MIT Press.

74. Martínez-Falero, E. \& González-Alonso, S., 1995. Quantitative Techniques in Landscape Planning. Boca Raton (Florida): Lewis Publishers, CRC Press Inc. .

75. Mauthner, F., Weiss, W. \& Spörk-Dür, M., 2016. Solar Heat Wordlwide. Markets and Contribution to the Energy Supply 2014. Austria: AEE-Institute for Sustainable Technologies.

76. Ministerio de Agricultura, 2012. Perú Forestal en Números Año 2011, Lima: Dirección General Forestal y de Fauna Silvestre.

77. Ministerio de Energía y Minas, 2008. Atlas Eólico del Perú. [En línea] Disponible en: http://www.osinergmin.gob.pe/seccion/centro documental/Institucional/Estudio s Economicos/Otros-Estudios/Atlas-Eolico/AtlasEolicoLibro.pdf [Último acceso: Febrero 2016].

78. Ministerio de Energía y Minas, 2010a. Decreto legislativo de promoción de la inversión para la generación de electricidad con el uso de energías renovables: Decreto Legislativo $\quad N^{o} 1002$. [En $\quad$ línea] Disponible en: http://www.minem.gob.pe/archivos/legislacion9ozj22z9ap5zz33z-

DL_de promocion_de la inversion_para_la_generacion_de electricidad_con_e 1 uso de energias renovables_1002.pdf

[Último acceso: Abril 2016].

79. Ministerio de Energía y Minas, 2010b. Estadística Eléctrica por Regiones 2010. [En línea] Disponible en: http://www.minem.gob.pe/minem/archivos/Cap\%C3\%83\%C2\%ADtulo2 $\% 20$ Estad $\%$ C3\%83\%C2\%ADstica $\% 20$ El $\%$ C3\%83\%C2\%A9ctrica $\% 20$ por $\% 20$ Regiones\%202010(1).pdf [Último acceso: Febrero 2016].

80. Ministerio de Energía y Minas, 2012. Plan Nacional de Electrificaación Rural 2013-2022. [En línea] Disponible en: http://dger.minem.gob.pe/ArchivosDger/PNER 20132022/PNER-2013-2022\%20Texto.pdf

[Último acceso: Febrero 2016]. 
81. Ministerio de Energía y Minas, 2014. Plan Energético Nacional 2014 - 2025. [En línea]

Disponible

en:

http://www.minem.gob.pe/minem/archivos/file/institucional/publicaciones/Infor mePlanEnerg\%C3\%ADa2025-\%20281114.pdf

[Último acceso: Enero 2016].

82. Ministerio de Salud, 2015. Análisis de Situación de Cajamarca. [En línea] Disponible en: $\quad$ dge.gob.pe/portal/Asis/indreg/asis_cajamarca.pdf [Último acceso: Noviembre 2017].

83. Ministerio del Ambiente, 2009. Proyecto Segunda Comunicación Nacional de Cambio Climático: Inventario Nacional Integrado de Gases de Efecto Invernadero del Perú en el Año 2000. [En línea] Disponible en: http://sinia.minam.gob.pe/documentos/inventario-nacionalintegrado-emisiones-gases-efecto-invernadero-peru

[Último acceso: Marzo 2016].

84. Mourmouris, J. C. \& Potolias, C., 2013. A multi-criteria methodology for energy planning and developing renewable energy sources at a regional level: A case study Thassos, Greece. Energy Policy, Issue 52, p. 522-530.

85. Naciones Unidas, 2015. Objetivos de Desarrollo Sostenible. [En línea] Disponible en: http://www.un.org/sustainabledevelopment/es/objetivos-dedesarrollo-sostenible/

[Último acceso: Octubre 2017].

86. Nerini, F., Howells, M., Bazilian, M. \& Gomez, M. F., 2014. Rural electrification options in the Brazilian Amazon. A multi-criteria analysis. Energy for Sustainable Development, Issue 20, p. 36-48.

87. Newkirk, R. T., 1979. Environmental planning for utility corridors. Michigan. United States: Ann Arbor Science.

88. Nijkamp, P., 1980. Environmental Policy Analysis. Operational Method and Models. Chichester: Wiley.

89. Orbegozo, C. \& Arivilca, R., 2010. Energía Solar Térmica. Manual Técnico para Termas Solares. Lima: Green Energy Consultoría y Servicios SRL.

90. Organismo Internacional de Energía Atómica, 2007. Modelo para el Análisis de la Demanda de Energía (MAED-2). Viena: OIEA.

91. Organización Latinoamericana de Energía, 2013. Modelos de Mercado, Regulación Económica y Tarifas del Sector Eléctrico en América Latina y el Caribe - Perú. [En línea] Disponible en: http://www.olade.org/wp-content/uploads/2015/08/InformeFinal-PERU.pdf

[Último acceso: Junio 2016].

92. OSINERGMIN, 2014a. Bases definitivas para la subasta de suministro de electricidad con recursos energéticos renovables en áreas no conectadas a red. 
[En

Disponible

http://www2.osinerg.gob.pe/EnergiasRenovables/contenido/Documentos/1Subas taOffGrid/Bases/BasesDefinitivasRevision.pdf

[Último acceso: Marzo 2015].

93. OSINERGMIN, 2014b. Reporte de Análisis Económico Sectorial Electricidad Año 3 - $N^{o}$ 4. Oficina de Estudios Económicos - OEE. [En línea] Disponible en: http://www.osinergmin.gob.pe/seccion/centro_documental/Institucional/Estudio s_Economicos/RAES/RAES_Electricidad_Noviembre_2014_OEE.pdf [Último acceso: Febrero 2016].

94. OSINERGMIN, 2015a. La industria de los hidrocarburos líquidos en el Perú: 20 años de aporte al desarrollo del país. Lima: s.n.

95. OSINERGMIN, 2015b. Balance Nacional de Energía 2014, desde la perspectiva de supervisor. [En línea] Disponible en: https://www.osinergmin.gob.pe/seccion/centro documental/hidrocarburos/Publi caciones/BALANCE\%20DE\%20ENERG\%C3\%8DA\%20EN\%20EL\%20PERU $\% 202014 . p d f$ [Último acceso: Abril 2016].

96. Otero, I., 1979. Análisis cuantitativo de los elementos del medio natural en orden a la planificación física. Madrid: Tesis doctoral inédita. Universidad Politécnica de Madrid.

97. Parodi, V., 2013. Propuesta metodológica para la evaluación integral de proyectos en el sector energético. Valencia: Tesis doctoral. Departamento de Proyectos de Ingeniería. Universidad Politécnica de Valencia.

98. Pérez, A., Montero, G. \& Ayala, R., 2015. Simulación en Aspen de la combustión de mezclas diesel-biodiesel. Ingeniería Investigación y Tecnología, XVI(1), pp. 83-92.

99. Phen, M., 2006. Dynamic life cycle assessment (LCA) of renewable energy technologies. Renewable Energy, Issue 31, p. 55-71.

100.Piriz, I., 2013. Energía solar térmica y fotovoltaica aislada para pequeñas comunidades en Perú. Barcelona: Tesis de Máster. ETSI de Caminos, Canales y Puertos. Universidad Politécnica de Cataluña.

101.Pohekar, S. D. \& Ramachandran, M., 2004. Application of multi-criteria decision making to sustainable energy planning-A review. Renewable and Sustainable Energy Reviews, Issue 8, p. 365-381.

102.Pontificia Universidad Católica del Perú, 2007. Atlas de Cajamarca. [En línea] Disponible en: http://www.atlascajamarca.info/provincial/cajamarca.html [Último acceso: Noviembre 2017]. 
103. Ramón, J. \& Cristóbal, C., 2012. A goal programming model for the optimal mix and location of renewable energy plants in the north of Spain. Renewable and Sustainable Energy Reviews, Volumen 16.

104. Real Academia de Ingeniería, 2011. Tecnologías para el desarrollo humano de las comunidades rurales aisladas. Madrid: RAI.

105.Rohit, S. \& Bhattacharyya, S. C., 2014. Off-grid electricity generation with renewable energy technologies in India: an application of HOMER. Renewable Energy, Issue 62, pp. 388-398.

106.Rojas-Zerpa, J. C. \& Yusta, J. M., 2014. Methodologies, technologies and applications for electric supply planning in rural remote areas. Energy for Sustainable Development, Issue 20, p. 66-76.

107. Romero, C., 1993. Teoría de la decisión multicriterio: Conceptos, técnicas y aplicaciones. Madrid: Alianza Universal Textos.

108. Romero, C., 1996. Análisis de las decisiones multicriterio. Madrid: Isdefe.

109. Roque, J., 2015. Sostenibilidad: desarrollo sostenible y equidad. Revista Redbioética / UNESCO, I(11), pp. 27-37.

110.Roy, B., 1968. Classement et choix en présence de points de vue multiples. R.I.R.O., 2(8), pp. 57-75.

111. Saaty, T. L., 1986. Decision Making for Leaders. The Analytical Hierarchy Process for Decisions in a Complex World. Pittsburgh, Pa., USA: RWS Publications.

112. Saaty, T. L., 2005a. The Analytic Hierarchy and Analytic Network Processes for the measurement of intangible criteria and for decision making. En: J. Figueira, S. Greco \& M. Ehrgott, edits. Multiple Criteria Decision Analysis: State of the Art Surveys. USA: Springer International Series.

113. Saaty, T. L., 2005b. Theory and Applicatios of the Analytic Network Process, Decision Making with Benefits, Opportunities, Costs and Risks. Pittsburgh, Pa., USA: RWS Publications.

114. Sánchez, M., 2013. Optimización con restricciones de desigualdad: Condiciones de Kuhn-Tucker. [En línea] Disponible en: https://classesenginyeries.wordpress.com/ [Último acceso: 30 Abril 2018].

115.Schrattenholzer, L., 1981. The Energy Supply Model MESSAGE. Laxenburg, Austria: IIASA Research Report.

116. Servicio Nacional de Meteorología e Hidrología, 2003. Atlas de Energía Solar del Perú.

Disponible en: http://dger.minem.gob.pe/atlassolar/ATLAS_SOLAR.pdf [Último acceso: Noviembre 2015]. 
117. Shapiro, J. F., White, D. E. \& Wood, D. O., 1976. Sensitivity analysis of the Brookhaven energy system optimization model, Durham: US Army Research Office.

118. Shtub, A., Bard, J. \& Globerson, S., 1994. Project Management. Engineering, Technology, and Implementation. New Jersey: Prentice Hall, Inc..

119. Silva, D. \& Nakata, T., 2009. Multi-objective assessment of rural electrification in remote areas with poverty considerations. Energy Policy, Issue 37, p. 30963108.

120. Simon, H. A., 1955. A Behavioral Model of Rational Choice. Quarterly Journal of Economics, Volumen 69.

121.Simon, H. A., 1957. Models of Man. Nueva York: John Wiley and Sons.

122.SNV, 2007. Línea de Base Biocombustibles en la Amazonia Peruana. Lima: Servicio Holandés de Cooperación al Desarrollo.

123. Soluciones Prácticas - ITDG, 2008. Microaerogeneradores de 100 y $500 \mathrm{~W}$. Modelos IT-PE-100 $y \quad$ SP-500. [En línea] Disponible en: $\quad$ www.solucionespracticas.org.pe/Descargar/1531/16285 [Último acceso: Febrero 2016].

124. Soluciones Prácticas - ITDG, 2010. Evaluación de Recursos Hidroenegéticos. Lima: Soluciones Prácticas - ITDG.

125. Soluciones Prácticas ITDG, 2015. Soluciones Prácticas en Cajamarca. [En línea] Disponible en: http://www.solucionespracticas.org.pe/ns_publicaciones [Último acceso: Abril 2016].

126. Soluciones Prácticas, 2012. BIP \#2 - Planificación y Desarrollo Energético. [En línea]

Disponible en: http://www.solucionespracticas.org.pe/ns_publicaciones [Último acceso: Febrero 2017].

127.Troldborg, M., Heslop, S. \& Hough, R. L., 2014. Assessing the sustainability of renewable energy technologies using multi-criteria analysis: Suitability of approach for national-scale assessments and associated uncertainties. Renewable and Sustainable Energy Reviews, Volumen 39, pp. 1173-1184.

128.U.S. Energy Information Administration, 2015. EIA. Today in Energy. [En línea] Disponible en: https://www.eia.gov/todayinenergy/detail.php?id=21912 [Último acceso: Enero 2018].

129. United Nations Development Programme, 2009. Human Development Report Peru.

Disponible en: http://hdr.undp.org/sites/default/files/idh2009-peru-vol1-2.pdf [Último acceso: Enero 2016].

130.Universidad Adolfo Ibañez; EECG Consultores, 2017. Planificación Energética de Largo Plazo - Ministerio de Energía de Chile. [En línea] 
Disponible

en:

http://pelp.minenergia.cl/

[Último acceso: 30 Abril 2018].

131.Unsihuay-Vila, C., Marangon-Lima, J. W., Zambroni de Souza, A. C. \& PerezArriaga, I. J., 2011. Multistage expansion planning of generation and interconnections with sustainable energy development criteria: A multiobjective model. Electrical Power and Energy Systems, Issue 33, pp. 258-270.

132. Vaillancourt, K., Labriet, M., Loulou, R. \& Waaub, J. P., 2008. The role of nuclear energy in long-term climate scenarios: An analysis with the World-TIMES model. Energy Policy, Issue 36, p. 2296-2307.

133. Velásquez, J., 2013. Memoria II Simposio Internacional de Energía Eólica de Pequeña Escala: Aportes en el desarrollo energético para América Latina. Lima, Soluciones Prácticas.

134.Wang, J.-J., Jing, Y.-Y., Zhang, C.-F. \& Zhao, J.-H., 2009. Review on multicriteria decision analysis aid in sustainable energy. Renewable and Sustainable Energy Reviews, Issue 13, p. 2263-2278.

135.World Bank, 2014. World Development Indicators. [En línea] Disponible en: http://databank.worldbank.org/data/reports.aspx?source=2\&type=metadata\&seri es=EG.ELC.ACCS.ZS\#advancedDownloadOptions [Último acceso: Octubre 2017].

136. Yadoo, A. \& Cruickshank, H., 2012. The role for low carbon electrification technologies in poverty reduction and climate change strategies: A focus on renewable energy mini-grids with case studies in Nepal, Peru and Kenya. Energy Policy, Issue 42, p. 591-602.

137.Yu, P. L., 1973. A Class of Solutions for Group Decision Problems. Management Science, Volumen 19.

138.Zadeh, L. A., 1963. Optimality and Non-scalar-Valued Perfomance Criteria. IEEE Transactions on Automatic Control, Volumen 8.

139.Zeleny, M., 1973. Compromise Programming. En: J. L. Cochrane \& M. Zeleny, edits. Multiple Criteria Decision Making. University of South Carolina Press: Columbia. 


\section{ANEXO I \\ DATOS OBTENIDOS DEL PROGRAMA MATLAB ${ }^{\circledR}$ PARA CADA ESCENARIO ANALIZADO}


Tabla A-I.1 Datos obtenidos para el escenario 1.

\begin{tabular}{|c|c|c|c|c|c|c|c|c|c|c|c|c|c|c|c|c|}
\hline \multirow{2}{*}{$\begin{array}{c}\text { Escenario } \\
\text { Pesos }\end{array}$} & \multicolumn{2}{|l|}{1} & \multicolumn{8}{|l|}{ Coordenadas } & \multicolumn{6}{|c|}{ Valor de la función } \\
\hline & Lk & Lk value & ST & PV & EOL & LEÑ & $\mathrm{CV}$ & BL & MH & GEO & F1 & F2 & F3 & F4 & F5 & F6 \\
\hline 1 & 1 & 0,7826 & 1,6542 & 2,8844 & 0,5126 & 12,6691 & 2,7010 & 1,0181 & 3,3275 & 0,2044 & 44,3452 & 163,3802 & 0,4085 & 0,6862 & 29996,2470 & 40,8133 \\
\hline 1 & 2 & 0,6533 & 1,5344 & 2,9291 & 0,2700 & 11,8238 & 2,5699 & 1,0079 & 3,5253 & 0,1925 & 42,7284 & 155,7091 & 0,3900 & 0,6542 & 28553,9432 & 39,0106 \\
\hline 1 & 3 & 0,5907 & 1,3902 & 2,8803 & 0,3201 & 10,8060 & 2,4121 & 0,9832 & 3,5240 & 0,1782 & 40,5982 & 146,5633 & 0,3680 & 0,6158 & 27396,1425 & 36,8960 \\
\hline 1 & Chebyshev & 0,4187 & 1,0655 & 2,7746 & 0,3675 & 8,5141 & 2,0567 & 0,9213 & 3,5823 & 0,1459 & 35,8275 & 125,9495 & 0,3186 & 0,5295 & 24628,0793 & 32,0993 \\
\hline 1 & & & & & & & & & & & & & & & & \\
\hline 1 & & & & & & & & & & & & & & & & \\
\hline
\end{tabular}

Tabla A-I.2 Datos obtenidos para el escenario 2.

\begin{tabular}{|c|c|c|c|c|c|c|c|c|c|c|c|c|c|c|c|c|}
\hline \multirow{2}{*}{$\begin{array}{l}\text { Escenario } \\
\text { Pesos }\end{array}$} & \multicolumn{2}{|l|}{2} & \multicolumn{8}{|l|}{ Coordenadas } & \multicolumn{6}{|c|}{ Valor de la función } \\
\hline & Lk & Lk value & ST & PV & EOL & LEÑ & CV & BL & MH & GEO & F1 & F2 & F3 & F4 & F5 & F6 \\
\hline 1 & 1 & 0 & 1,6576 & 3,2376 & 0,0129 & 12,6934 & 2,7048 & 1,0344 & 3,4738 & 0,2048 & 44,6611 & 163,2599 & 0,4078 & 0,6862 & 29712,7320 & 40,9825 \\
\hline 0 & 2 & 0 & 1,6576 & 3,2376 & 0,0129 & 12,6934 & 2,7048 & 1,0344 & 3,4738 & 0,2048 & 44,6611 & 163,2599 & 0,4078 & 0,6862 & 29712,7320 & 40,9825 \\
\hline 0 & 3 & 0 & 1,6576 & 3,2376 & 0,0129 & 12,6934 & 2,7048 & 1,0344 & 3,4738 & 0,2048 & 44,6611 & 163,2599 & 0,4078 & 0,6862 & 29712,7320 & 40,9825 \\
\hline 0 & Chebyshev & 0 & 1,6576 & 3,2376 & 0,0129 & 12,6934 & 2,7048 & 1,0344 & 3,4738 & 0,2048 & 44,6611 & 163,2599 & 0,4078 & 0,6862 & 29712,7320 & 40,9825 \\
\hline 0 & & & & & & & & & & & & & & & & \\
\hline 0 & & & & & & & & & & & & & & & & \\
\hline
\end{tabular}

Tabla A-I.3 Datos obtenidos para el escenario 3.

\begin{tabular}{|c|c|c|c|c|c|c|c|c|c|c|c|c|c|c|c|c|}
\hline \multirow{2}{*}{$\begin{array}{l}\text { Escenario } \\
\text { Pesos }\end{array}$} & \multicolumn{2}{|l|}{3} & \multicolumn{8}{|l|}{ Coordenadas } & \multicolumn{6}{|c|}{ Valor de la función } \\
\hline & Lk & Lk value & ST & PV & EOL & LEÑ & CV & BL & MH & GEO & F1 & F2 & F3 & F4 & F5 & F6 \\
\hline 5 & 1 & 0,7934 & 1,6557 & 3,1236 & 0,2185 & 12,6797 & 2,7026 & 1,0292 & 3,3823 & 0,2046 & 44,5249 & 163,2471 & 0,4079 & 0,6860 & 29909,4902 & 40,9380 \\
\hline 1 & 2 & 0,6752 & 1,6393 & 3,0968 & 0,1464 & 12,5638 & 2,6847 & 1,0289 & 3,4812 & 0,2029 & 44,3238 & 162,2291 & 0,4055 & 0,6817 & 29527,8714 & 40,6396 \\
\hline 1 & 3 & 0,6596 & 1,5803 & 3,1238 & 0,1315 & 12,1479 & 2,6202 & 1,0195 & 3,4691 & 0,1971 & 43,4718 & 158,4452 & 0,3963 & 0,6659 & 29090,2429 & 39,8055 \\
\hline 1 & Chebyshev & 0,6031 & 1,4745 & 3,0837 & 0,1886 & 11,4010 & 2,5043 & 0,9990 & 3,4521 & 0,1866 & 41,8959 & 151,7338 & 0,3802 & 0,6378 & 28278,0764 & 38,2572 \\
\hline 1 & & & & & & & & & & & & & & & & \\
\hline 1 & & & & & & & & & & & & & & & & \\
\hline
\end{tabular}


Tabla A-I.4 Datos obtenidos para el escenario 4.

\begin{tabular}{|c|c|c|c|c|c|c|c|c|c|c|c|c|c|c|c|c|}
\hline Escenario & 4 & & Coordenadas & & & & & & & & $\begin{array}{l}\text { Valor de la } \\
\text { función }\end{array}$ & & & & & \\
\hline Pesos & Lk & Lk value & ST & PV & EOL & LEÑ & CV & BL & $\mathrm{MH}$ & GEO & F1 & F2 & F3 & F4 & F5 & F6 \\
\hline 0 & 1 & 0 & 1,6576 & 2,6440 & 2,3935 & 12,6934 & 2,7048 & 1,0344 & 1,6870 & 0,2048 & 43,4708 & 163,9311 & 0,4103 & 0,6877 & 34152,4188 & 41,5791 \\
\hline 1 & 2 & 0 & 1,6576 & 2,6440 & 2,3935 & 12,6934 & 2,7048 & 1,0344 & 1,6870 & 0,2048 & 43,4708 & 163,9311 & 0,4103 & 0,6877 & 34152,4188 & 41,5791 \\
\hline 0 & 3 & 0 & 1,6576 & 2,6440 & 2,3935 & 12,6934 & 2,7048 & 1,0344 & 1,6870 & 0,2048 & 43,4708 & 163,9311 & 0,4103 & 0,6877 & 34152,4188 & 41,5791 \\
\hline 0 & Chebyshev & 0 & 1,6576 & 2,6440 & 2,3935 & 12,6934 & 2,7048 & 1,0344 & 1,6870 & 0,2048 & 43,4708 & 163,9311 & 0,4103 & 0,6877 & 34152,4188 & 41,5791 \\
\hline 0 & & & & & & & & & & & & & & & & \\
\hline 0 & & & & & & & & & & & & & & & & \\
\hline
\end{tabular}

Tabla A-I.5 Datos obtenidos para el escenario 5.

\begin{tabular}{|c|c|c|c|c|c|c|c|c|c|c|c|c|c|c|c|c|}
\hline Escenario & 5 & & Coordenadas & & & & & & & & $\begin{array}{l}\text { Valor de la } \\
\text { función }\end{array}$ & & & & & \\
\hline Pesos & Lk & Lk value & ST & PV & EOL & LEÑ & CV & BL & MH & GEO & F1 & F2 & F3 & F4 & F5 & F6 \\
\hline 1 & 1 & 0,8429 & 1,6513 & 2,9573 & 0,6118 & 12,6486 & 2,6978 & 1,0326 & 3,1554 & 0,2041 & 44,2682 & 163,1533 & 0,4079 & 0,6853 & 30432,0741 & 40,9088 \\
\hline 5 & 2 & 0,7086 & 1,6001 & 2,8709 & 0,5280 & 12,2875 & 2,6418 & 1,0109 & 3,3255 & 0,1990 & 43,5502 & 159,9498 & 0,4003 & 0,6718 & 29568,5688 & 40,0256 \\
\hline 1 & 3 & 0,6612 & 1,5814 & 2,9342 & 0,3366 & 12,1553 & 2,6213 & 1,0146 & 3,4536 & 0,1972 & 43,3794 & 158,6987 & 0,3972 & 0,6667 & 29110,1445 & 39,7285 \\
\hline 1 & Chebyshev & 0,6258 & 1,4701 & 2,8242 & 0,6532 & 11,3701 & 2,4995 & 0,9899 & 3,2470 & 0,1861 & 41,5914 & 151,7138 & 0,3805 & 0,6372 & 28721,3232 & 38,1582 \\
\hline 1 & & & & & & & & & & & & & & & & \\
\hline 1 & & & & & & & & & & & & & & & & \\
\hline
\end{tabular}

Tabla A-I.6 Datos obtenidos para el escenario 6.

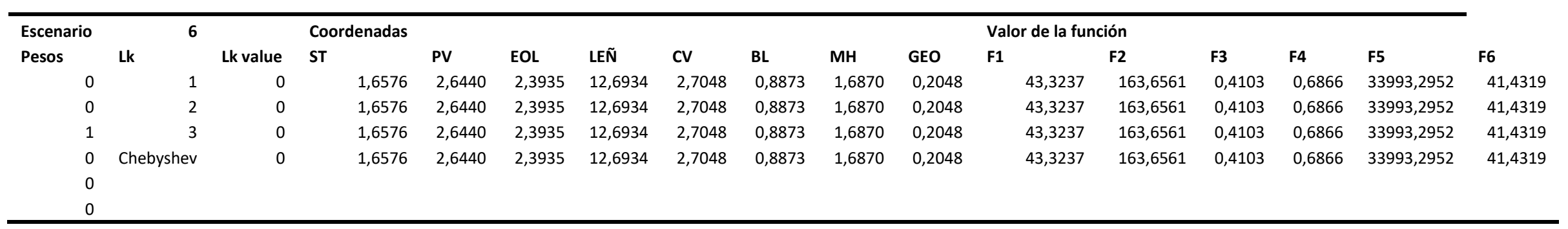


Tabla A-I.7 Datos obtenidos para el escenario 7.

\begin{tabular}{|c|c|c|c|c|c|c|c|c|c|c|c|c|c|c|c|c|}
\hline Escenario & 7 & & Coordenadas & & & & & & & & $\begin{array}{l}\text { Valor de la } \\
\text { función }\end{array}$ & & & & & \\
\hline Pesos & Lk & Lk value & ST & PV & EOL & LEÑ & CV & BL & $\mathrm{MH}$ & GEO & F1 & F2 & F3 & F4 & F5 & F6 \\
\hline 1 & 1 & 0,8394 & 1,6558 & 2,9056 & 0,7801 & 12,6808 & 2,7028 & 1,0323 & 3,0387 & 0,2046 & 44,2496 & 163,4995 & 0,4088 & 0,6866 & 30755,7601 & 41,0063 \\
\hline 1 & 2 & 0,6990 & 1,6146 & 2,8730 & 0,5004 & 12,3896 & 2,6576 & 1,0180 & 3,3510 & 0,2005 & 43,7797 & 160,8778 & 0,4025 & 0,6757 & 29625,5261 & 40,2283 \\
\hline 5 & 3 & 0,6758 & 1,6146 & 2,8730 & 0,5004 & 12,3896 & 2,6576 & 1,0180 & 3,3510 & 0,2005 & 43,7797 & 160,8778 & 0,4025 & 0,6757 & 29625,5261 & 40,2283 \\
\hline 1 & Chebyshev & 0,6056 & 1,4800 & 2,8452 & 0,4425 & 11,4396 & 2,5103 & 0,9941 & 3,4367 & 0,1871 & 41,8429 & 152,3186 & 0,3819 & 0,6399 & 28326,6894 & 38,2191 \\
\hline 1 & & & & & & & & & & & & & & & & \\
\hline 1 & & & & & & & & & & & & & & & & \\
\hline
\end{tabular}

Tabla A-I.8 Datos obtenidos para el escenario 8.

\begin{tabular}{|c|c|c|c|c|c|c|c|c|c|c|c|c|c|c|c|c|}
\hline Escenario & 8 & & Coordenadas & & & & & & & & $\begin{array}{l}\text { Valor de la } \\
\text { función }\end{array}$ & & & & & \\
\hline Pesos & Lk & Lk value & ST & PV & EOL & LEÑ & CV & BL & MH & GEO & F1 & F2 & F3 & F4 & F5 & F6 \\
\hline 0 & 1 & 0 & 1,6576 & 2,6440 & 0,3552 & 12,6934 & 2,7048 & 1,0344 & 3,7253 & 0,2048 & 44,4900 & 163,8647 & 0,4099 & 0,6879 & 29009,2749 & 40,5599 \\
\hline 0 & 2 & 0 & 1,6576 & 2,6440 & 0,3552 & 12,6934 & 2,7048 & 1,0344 & 3,7253 & 0,2048 & 44,4900 & 163,8647 & 0,4099 & 0,6879 & 29009,2749 & 40,5599 \\
\hline 0 & 3 & 0 & 1,6576 & 2,6440 & 0,3552 & 12,6934 & 2,7048 & 1,0344 & 3,7253 & 0,2048 & 44,4900 & 163,8647 & 0,4099 & 0,6879 & 29009,2749 & 40,5599 \\
\hline 1 & Chebyshev & 0 & 1,6576 & 2,6440 & 0,3552 & 12,6934 & 2,7048 & 1,0344 & 3,7253 & 0,2048 & 44,4900 & 163,8647 & 0,4099 & 0,6879 & 29009,2749 & 40,5599 \\
\hline 0 & & & & & & & & & & & & & & & & \\
\hline 0 & & & & & & & & & & & & & & & & \\
\hline
\end{tabular}

Tabla A-I.9 Datos obtenidos para el escenario 9.

\begin{tabular}{|c|c|c|c|c|c|c|c|c|c|c|c|c|c|c|c|c|}
\hline Escenario & 9 & & Coordenadas & & & & & & & & $\begin{array}{l}\text { Valor de la } \\
\text { función }\end{array}$ & & & & & \\
\hline Pesos & Lk & Lk value & ST & PV & EOL & LEÑ & CV & BL & $\mathrm{MH}$ & GEO & F1 & F2 & F3 & F4 & F5 & F6 \\
\hline 1 & 1 & 0,7430 & 1,6572 & 2,7535 & 0,3697 & 12,6902 & 2,7043 & 1,0326 & 3,6012 & 0,2047 & 44,4744 & 163,7236 & 0,4094 & 0,6875 & 29329,6169 & 40,6685 \\
\hline 1 & 2 & 0,6530 & 1,6403 & 2,7101 & 0,3778 & 12,5714 & 2,6858 & 1,0295 & 3,6365 & 0,2030 & 44,2242 & 162,6928 & 0,4070 & 0,6831 & 29100,0965 & 40,3846 \\
\hline 1 & 3 & 0,6299 & 1,5885 & 2,6956 & 0,3540 & 12,2057 & 2,6291 & 1,0203 & 3,6749 & 0,1979 & 43,4795 & 159,4017 & 0,3991 & 0,6693 & 28586,3134 & 39,6067 \\
\hline 5 & Chebyshev & 0,5773 & 1,4847 & 2,6778 & 0,3821 & 11,4726 & 2,5154 & 0,9992 & 3,6645 & 0,1876 & 41,9457 & 152,7904 & 0,3832 & 0,6416 & 27774,5149 & 38,0936 \\
\hline 1 & & & & & & & & & & & & & & & & \\
\hline 1 & & & & & & & & & & & & & & & & \\
\hline
\end{tabular}


Tabla A-I.10 Datos obtenidos para el escenario 10.

\begin{tabular}{|c|c|c|c|c|c|c|c|c|c|c|c|c|c|c|c|c|}
\hline Escenario & 10 & & Coordenadas & & & & & & & & $\begin{array}{l}\text { Valor de la } \\
\text { función }\end{array}$ & & & & & \\
\hline Pesos & Lk & Lk value & ST & PV & EOL & LEÑ & cV & BL & MH & GEO & F1 & F2 & F3 & F4 & F5 & F6 \\
\hline 0 & 1 & 0 & 0,1000 & 2,6440 & 0,3552 & 1,7000 & 1,0000 & 0,7401 & 3,7253 & 0,0500 & 21,7236 & 64,4808 & 0,1709 & 0,2721 & 16499,6646 & 17,9483 \\
\hline 0 & 2 & 0 & 0,1000 & 2,6440 & 0,3552 & 1,7000 & 1,0000 & 0,7401 & 3,7253 & 0,0500 & 21,7236 & 64,4808 & 0,1709 & 0,2721 & 16499,6646 & 17,9483 \\
\hline 0 & 3 & 0 & 0,1000 & 2,6440 & 0,3552 & 1,7000 & 1,0000 & 0,7401 & 3,7253 & 0,0500 & 21,7236 & 64,4808 & 0,1709 & 0,2721 & 16499,6646 & 17,9483 \\
\hline 0 & Chebyshev & 0 & 0,1000 & 2,6440 & 0,3552 & 1,7000 & 1,0000 & 0,7401 & 3,7253 & 0,0500 & 21,7236 & 64,4808 & 0,1709 & 0,2721 & 16499,6646 & 17,9483 \\
\hline 1 & & & & & & & & & & & & & & & & \\
\hline 0 & & & & & & & & & & & & & & & & \\
\hline
\end{tabular}

Tabla A-I.11 Datos obtenidos para el escenario 11.

\begin{tabular}{|c|c|c|c|c|c|c|c|c|c|c|c|c|c|c|c|c|}
\hline Escenario & 11 & & Coordenadas & & & & & & & & $\begin{array}{l}\text { Valor de la } \\
\text { función }\end{array}$ & & & & & \\
\hline Pesos & Lk & Lk value & ST & PV & EOL & LEÑ & CV & BL & MH & GEO & F1 & F2 & F3 & F4 & F5 & F6 \\
\hline 1 & 1 & 3,5515 & 1,6383 & 2,7835 & 0,5932 & 12,5572 & 2,6836 & 1,0296 & 3,3477 & 0,2028 & 44,0876 & 162,4989 & 0,4065 & 0,6823 & 29821,7939 & 40,5370 \\
\hline 1 & 2 & 1,8705 & 0,5407 & 2,7219 & 0,3361 & 4,8100 & 1,4823 & 0,8232 & 3,6664 & 0,0938 & 28,1735 & 92,5176 & 0,2382 & 0,3895 & 20196,0159 & 24,4133 \\
\hline 1 & 3 & 1,3930 & 0,4927 & 2,7197 & 0,3155 & 4,4715 & 1,4298 & 0,8142 & 3,6892 & 0,0890 & 27,4829 & 89,4590 & 0,2309 & 0,3767 & 19753,0580 & 23,7047 \\
\hline 1 & Chebyshev & 0,7885 & 0,4500 & 2,6978 & 0,3923 & 4,1699 & 1,3830 & 0,8025 & 3,6343 & 0,0848 & 26,8163 & 86,7502 & 0,2244 & 0,3654 & 19541,9560 & 23,0972 \\
\hline 5 & & & & & & & & & & & & & & & & \\
\hline 1 & & & & & & & & & & & & & & & & \\
\hline
\end{tabular}

Tabla A-I.12 Datos obtenidos para el escenario 12.

\begin{tabular}{|c|c|c|c|c|c|c|c|c|c|c|c|c|c|c|c|c|}
\hline Escenario & 12 & & Coordenadas & & & & & & & & $\begin{array}{l}\text { Valor de la } \\
\text { función }\end{array}$ & & & & & \\
\hline \multirow[t]{7}{*}{ Pesos } & Lk & Lk value & ST & PV & EOL & LEÑ & CV & BL & $\mathrm{MH}$ & GEO & F1 & F2 & F3 & F4 & F5 & F6 \\
\hline & 1 & 0 & 1,6576 & 3,3552 & 2,3935 & 12,6934 & 2,7048 & 1,0344 & 0,9758 & 0,2048 & 43,4708 & 163,2199 & 0,4079 & 0,6855 & 36029,6489 & 42,2903 \\
\hline & 2 & 0 & 1,6576 & 3,3552 & 2,3935 & 12,6934 & 2,7048 & 1,0344 & 0,9758 & 0,2048 & 43,4708 & 163,2199 & 0,4079 & 0,6855 & 36029,6489 & 42,2903 \\
\hline & 3 & 0 & 1,6576 & 3,3552 & 2,3935 & 12,6934 & 2,7048 & 1,0344 & 0,9758 & 0,2048 & 43,4708 & 163,2199 & 0,4079 & 0,6855 & 36029,6489 & 42,2903 \\
\hline & Chebyshev & 0 & 1,6576 & 3,3552 & 2,3935 & 12,6934 & 2,7048 & 1,0344 & 0,9758 & 0,2048 & 43,4708 & 163,2199 & 0,4079 & 0,6855 & 36029,6489 & 42,2903 \\
\hline & & & & & & & & & & & & & & & & \\
\hline & & & & & & & & & & & & & & & & \\
\hline
\end{tabular}


Tabla A-I.13 Datos obtenidos para el escenario 13.

\begin{tabular}{|c|c|c|c|c|c|c|c|c|c|c|c|c|c|c|c|c|}
\hline Escenario & 13 & & Coordenadas & & & & & & & & $\begin{array}{l}\text { Valor de la } \\
\text { función }\end{array}$ & & & & & \\
\hline Pesos & Lk & Lk value & ST & PV & EOL & LEÑ & CV & BL & $\mathrm{MH}$ & GEO & F1 & F2 & F3 & F4 & F5 & F6 \\
\hline 1 & 1 & 1,0161 & 1,6552 & 2,9541 & 0,5668 & 12,6766 & 2,7021 & 1,0338 & 3,2035 & 0,2045 & 44,3491 & 163,4089 & 0,4085 & 0,6863 & 30342,4654 & 40,9411 \\
\hline 1 & 2 & 0,7612 & 1,6552 & 2,9541 & 0,5668 & 12,6766 & 2,7021 & 1,0338 & 3,2035 & 0,2045 & 44,3491 & 163,4089 & 0,4085 & 0,6863 & 30342,4654 & 40,9411 \\
\hline 1 & 3 & 0,7205 & 1,6069 & 2,8871 & 0,3611 & 12,3351 & 2,6492 & 1,0238 & 3,4763 & 0,1997 & 43,7439 & 160,3805 & 0,4012 & 0,6737 & 29256,9609 & 40,0680 \\
\hline 1 & Chebyshev & 0,6365 & 1,5552 & 2,8168 & 0,4730 & 11,9704 & 2,5926 & 1,0068 & 3,4346 & 0,1946 & 42,9253 & 157,1434 & 0,3936 & 0,6600 & 28931,1415 & 39,2962 \\
\hline 1 & & & & & & & & & & & & & & & & \\
\hline 5 & & & & & & & & & & & & & & & & \\
\hline
\end{tabular}

Tabla A-I.14 Datos obtenidos para el escenario 14.

\begin{tabular}{|c|c|c|c|c|c|c|c|c|c|c|c|c|c|c|c|c|}
\hline Escenario & 14 & & Coordenadas & & & & & & & & $\begin{array}{l}\text { Valor de la } \\
\text { función }\end{array}$ & & & & & \\
\hline \multirow[t]{7}{*}{ Pesos } & Lk & Lk value & ST & PV & EOL & LEÑ & CV & BL & MH & GEO & F1 & F2 & F3 & F4 & F5 & F6 \\
\hline & 1 & 0,7820 & 1,6575 & 2,6936 & 0,6451 & 12,6924 & 2,7046 & 1,0333 & 3,3857 & 0,2047 & 44,3418 & 163,8133 & 0,4097 & 0,6877 & 29869,4464 & 40,7513 \\
\hline & 2 & 0,6631 & 1,6463 & 2,7637 & 0,3701 & 12,6134 & 2,6923 & 1,0309 & 3,5906 & 0,2036 & 44,3152 & 163,0188 & 0,4077 & 0,6845 & 29270,4429 & 40,5211 \\
\hline & 3 & 0,6545 & 1,6463 & 2,7637 & 0,3701 & 12,6134 & 2,6923 & 1,0309 & 3,5906 & 0,2036 & 44,3152 & 163,0188 & 0,4077 & 0,6845 & 29270,4429 & 40,5211 \\
\hline & Chebyshev & 0,5864 & 1,4865 & 2,6877 & 0,4362 & 11,4855 & 2,5174 & 0,9990 & 3,6006 & 0,1878 & 41,9448 & 152,8975 & 0,3835 & 0,6421 & 27951,0867 & 38,1565 \\
\hline & & & & & & & & & & & & & & & & \\
\hline & & & & & & & & & & & & & & & & \\
\hline
\end{tabular}

Tabla A-I.15 Datos obtenidos para el escenario 15.

\begin{tabular}{|c|c|c|c|c|c|c|c|c|c|c|c|c|c|c|c|c|}
\hline Escenario & 15 & & Coordenadas & & & & & & & & $\begin{array}{l}\text { Valor de la } \\
\text { función }\end{array}$ & & & & & \\
\hline Pesos & Lk & Lk value & ST & PV & EOL & LEÑ & CV & BL & $\mathrm{MH}$ & GEO & F1 & F2 & F3 & F4 & F5 & F6 \\
\hline 5 & 1 & 1,0123 & 1,6568 & 3,2008 & 0,2926 & 12,6877 & 2,7039 & 1,0333 & 3,2311 & 0,2047 & 44,5085 & 163,2525 & 0,4078 & 0,6861 & 30313,4618 & 41,0728 \\
\hline 1 & 2 & 0,7418 & 1,6532 & 3,0693 & 0,2261 & 12,6623 & 2,6999 & 1,0300 & 3,4290 & 0,2043 & 44,4864 & 163,1469 & 0,4077 & 0,6855 & 29766,8611 & 40,8531 \\
\hline 1 & 3 & 0,6947 & 1,6373 & 3,0599 & 0,1602 & 12,5502 & 2,6825 & 1,0305 & 3,5044 & 0,2027 & 44,2907 & 162,1474 & 0,4053 & 0,6813 & 29451,8536 & 40,5836 \\
\hline 1 & Chebyshev & 0,6326 & 1,5408 & 3,0813 & 0,2106 & 11,8687 & 2,5769 & 1,0064 & 3,4325 & 0,1932 & 42,8483 & 155,9555 & 0,3904 & 0,6554 & 28853,9413 & 39,2227 \\
\hline 1 & & & & & & & & & & & & & & & & \\
\hline 5 & & & & & & & & & & & & & & & & \\
\hline
\end{tabular}




\section{ANEXO II}

DATOS OBTENIDOS POR VARIABLE Y FUNCIÓN OBJETIVO 
Tabla A-II.1 Datos obtenidos para la variable ST (ktep).

\begin{tabular}{rrrrrrcccc}
\hline \multicolumn{1}{c}{ Pesos } & & & \multicolumn{3}{c}{ VARIACIÓN DE ST } \\
F1 & F2 & F3 & F4 & F5 & F6 & L1 & L2 & L3 & Lo \\
1 & 1 & 1 & 1 & 1 & 1 & 1,654 & 1,534 & 1,390 & 1,065 \\
1 & 0 & 0 & 0 & 0 & 0 & 1,658 & 1,658 & 1,658 & 1,658 \\
5 & 1 & 1 & 1 & 1 & 1 & 1,656 & 1,639 & 1,580 & 1,475 \\
0 & 1 & 0 & 0 & 0 & 0 & 1,658 & 1,658 & 1,658 & 1,658 \\
1 & 5 & 1 & 1 & 1 & 1 & 1,651 & 1,600 & 1,581 & 1,470 \\
0 & 0 & 1 & 0 & 0 & 0 & 1,658 & 1,658 & 1,658 & 1,658 \\
1 & 1 & 5 & 1 & 1 & 1 & 1,656 & 1,615 & 1,615 & 1,480 \\
0 & 0 & 0 & 1 & 0 & 0 & 1,658 & 1,658 & 1,658 & 1,658 \\
1 & 1 & 1 & 5 & 1 & 1 & 1,657 & 1,640 & 1,589 & 1,485 \\
0 & 0 & 0 & 0 & 1 & 0 & 0,100 & 0,100 & 0,100 & 0,100 \\
1 & 1 & 1 & 1 & 5 & 1 & 1,638 & 0,541 & 0,493 & 0,450 \\
0 & 0 & 0 & 0 & 0 & 1 & 1,658 & 1,658 & 1,658 & 1,658 \\
1 & 1 & 1 & 1 & 1 & 5 & 1,655 & 1,655 & 1,607 & 1,555 \\
1 & 5 & 5 & 5 & 1 & 1 & 1,657 & 1,646 & 1,646 & 1,486 \\
5 & 1 & 1 & 1 & 1 & 5 & 1,657 & 1,653 & 1,637 & 1,541 \\
\hline
\end{tabular}

Tabla A-II.2 Datos obtenidos para la variable PV (ktep).

\begin{tabular}{rrrrrrcccc}
\hline \multicolumn{1}{c}{ Pesos } & & & \multicolumn{3}{c}{ VARIACIÓN DE PV } \\
F1 & F2 & F3 & F4 & F5 & F6 & L1 & L2 & L3 & L \\
1 & 1 & 1 & 1 & 1 & 1 & 2,884 & 2,929 & 2,880 & 2,775 \\
1 & 0 & 0 & 0 & 0 & 0 & 3,238 & 3,238 & 3,238 & 3,238 \\
5 & 1 & 1 & 1 & 1 & 1 & 3,124 & 3,097 & 3,124 & 3,084 \\
0 & 1 & 0 & 0 & 0 & 0 & 2,644 & 2,644 & 2,644 & 2,644 \\
1 & 5 & 1 & 1 & 1 & 1 & 2,957 & 2,871 & 2,934 & 2,824 \\
0 & 0 & 1 & 0 & 0 & 0 & 2,644 & 2,644 & 2,644 & 2,644 \\
1 & 1 & 5 & 1 & 1 & 1 & 2,906 & 2,873 & 2,873 & 2,845 \\
0 & 0 & 0 & 1 & 0 & 0 & 2,644 & 2,644 & 2,644 & 2,644 \\
1 & 1 & 1 & 5 & 1 & 1 & 2,753 & 2,710 & 2,696 & 2,678 \\
0 & 0 & 0 & 0 & 1 & 0 & 2,644 & 2,644 & 2,644 & 2,644 \\
1 & 1 & 1 & 1 & 5 & 1 & 2,784 & 2,722 & 2,720 & 2,698 \\
0 & 0 & 0 & 0 & 0 & 1 & 3,355 & 3,355 & 3,355 & 3,355 \\
1 & 1 & 1 & 1 & 1 & 5 & 2,954 & 2,954 & 2,887 & 2,817 \\
1 & 5 & 5 & 5 & 1 & 1 & 2,694 & 2,764 & 2,764 & 2,688 \\
5 & 1 & 1 & 1 & 1 & 5 & 3,201 & 3,069 & 3,060 & 3,081 \\
\hline
\end{tabular}


Tabla A-II.3 Datos obtenidos para la variable EOL (ktep).

\begin{tabular}{rrrrrrcccc}
\hline \multicolumn{1}{c}{ Pesos } & F2 & F3 & F4 & F5 & F6 & L1 & L2 & L3 & Lo \\
1 & 1 & 1 & 1 & 1 & 1 & 0,513 & 0,270 & 0,320 & 0,367 \\
1 & 0 & 0 & 0 & 0 & 0 & 0,013 & 0,013 & 0,013 & 0,013 \\
5 & 1 & 1 & 1 & 1 & 1 & 0,219 & 0,146 & 0,131 & 0,189 \\
0 & 1 & 0 & 0 & 0 & 0 & 2,393 & 2,393 & 2,393 & 2,393 \\
1 & 5 & 1 & 1 & 1 & 1 & 0,612 & 0,528 & 0,337 & 0,653 \\
0 & 0 & 1 & 0 & 0 & 0 & 2,393 & 2,393 & 2,393 & 2,393 \\
1 & 1 & 5 & 1 & 1 & 1 & 0,780 & 0,500 & 0,500 & 0,443 \\
0 & 0 & 0 & 1 & 0 & 0 & 0,355 & 0,355 & 0,355 & 0,355 \\
1 & 1 & 1 & 5 & 1 & 1 & 0,370 & 0,378 & 0,354 & 0,382 \\
0 & 0 & 0 & 0 & 1 & 0 & 0,355 & 0,355 & 0,355 & 0,355 \\
1 & 1 & 1 & 1 & 5 & 1 & 0,593 & 0,336 & 0,315 & 0,392 \\
0 & 0 & 0 & 0 & 0 & 1 & 2,393 & 2,393 & 2,393 & 2,393 \\
1 & 1 & 1 & 1 & 1 & 5 & 0,567 & 0,567 & 0,361 & 0,473 \\
1 & 5 & 5 & 5 & 1 & 1 & 0,645 & 0,370 & 0,370 & 0,436 \\
5 & 1 & 1 & 1 & 1 & 5 & 0,293 & 0,226 & 0,160 & 0,211 \\
\hline
\end{tabular}

Tabla A-II.4 Datos obtenidos para la variable LEÑ (ktep).

\begin{tabular}{rrrrrrcccc}
\hline \multicolumn{1}{c}{ Pesos } & & & \multicolumn{4}{c}{ VARIACIÓN DE LEN } \\
F1 & F2 & F3 & F4 & F5 & F6 & L1 & L2 & L3 & Lo \\
1 & 1 & 1 & 1 & 1 & 1 & 12,669 & 11,824 & 10,806 & 8,514 \\
1 & 0 & 0 & 0 & 0 & 0 & 12,693 & 12,693 & 12,693 & 12,693 \\
5 & 1 & 1 & 1 & 1 & 1 & 12,680 & 12,564 & 12,148 & 11,401 \\
0 & 1 & 0 & 0 & 0 & 0 & 12,693 & 12,693 & 12,693 & 12,693 \\
1 & 5 & 1 & 1 & 1 & 1 & 12,649 & 12,287 & 12,155 & 11,370 \\
0 & 0 & 1 & 0 & 0 & 0 & 12,693 & 12,693 & 12,693 & 12,693 \\
1 & 1 & 5 & 1 & 1 & 1 & 12,681 & 12,390 & 12,390 & 11,440 \\
0 & 0 & 0 & 1 & 0 & 0 & 12,693 & 12,693 & 12,693 & 12,693 \\
1 & 1 & 1 & 5 & 1 & 1 & 12,690 & 12,571 & 12,206 & 11,473 \\
0 & 0 & 0 & 0 & 1 & 0 & 1,700 & 1,700 & 1,700 & 1,700 \\
1 & 1 & 1 & 1 & 5 & 1 & 12,557 & 4,810 & 4,472 & 4,170 \\
0 & 0 & 0 & 0 & 0 & 1 & 12,693 & 12,693 & 12,693 & 12,693 \\
1 & 1 & 1 & 1 & 1 & 5 & 12,677 & 12,677 & 12,335 & 11,970 \\
1 & 5 & 5 & 5 & 1 & 1 & 12,692 & 12,613 & 12,613 & 11,485 \\
5 & 1 & 1 & 1 & 1 & 5 & 12,688 & 12,662 & 12,550 & 11,869 \\
\hline
\end{tabular}


Tabla A-II.5 Datos obtenidos para la variable CV (ktep).

\begin{tabular}{rrrrrrcccc}
\hline \multicolumn{1}{c}{ Pesos } & \multicolumn{1}{c}{ VARIACIÓN DE CV } \\
F1 & F2 & F3 & F4 & F5 & F6 & L1 & L2 & L3 & L \\
1 & 1 & 1 & 1 & 1 & 1 & 2,701 & 2,570 & 2,412 & 2,057 \\
1 & 0 & 0 & 0 & 0 & 0 & 2,705 & 2,705 & 2,705 & 2,705 \\
5 & 1 & 1 & 1 & 1 & 1 & 2,703 & 2,685 & 2,620 & 2,504 \\
0 & 1 & 0 & 0 & 0 & 0 & 2,705 & 2,705 & 2,705 & 2,705 \\
1 & 5 & 1 & 1 & 1 & 1 & 2,698 & 2,642 & 2,621 & 2,500 \\
0 & 0 & 1 & 0 & 0 & 0 & 2,705 & 2,705 & 2,705 & 2,705 \\
1 & 1 & 5 & 1 & 1 & 1 & 2,703 & 2,658 & 2,658 & 2,510 \\
0 & 0 & 0 & 1 & 0 & 0 & 2,705 & 2,705 & 2,705 & 2,705 \\
1 & 1 & 1 & 5 & 1 & 1 & 2,704 & 2,686 & 2,629 & 2,515 \\
0 & 0 & 0 & 0 & 1 & 0 & 1,000 & 1,000 & 1,000 & 1,000 \\
1 & 1 & 1 & 1 & 5 & 1 & 2,684 & 1,482 & 1,430 & 1,383 \\
0 & 0 & 0 & 0 & 0 & 1 & 2,705 & 2,705 & 2,705 & 2,705 \\
1 & 1 & 1 & 1 & 1 & 5 & 2,702 & 2,702 & 2,649 & 2,593 \\
1 & 5 & 5 & 5 & 1 & 1 & 2,705 & 2,692 & 2,692 & 2,517 \\
5 & 1 & 1 & 1 & 1 & 5 & 2,704 & 2,700 & 2,683 & 2,577 \\
\hline
\end{tabular}

Tabla A-II.6 Datos obtenidos para la variable BL (ktep).

\begin{tabular}{rrrrrrcccc}
\hline \multicolumn{1}{c}{ Pesos } & & & \multicolumn{3}{c}{ VARIACIÓN DE BL } \\
F1 & F2 & F3 & F4 & F5 & F6 & L1 & L2 & L3 & Lo \\
1 & 1 & 1 & 1 & 1 & 1 & 1,018 & 1,008 & 0,983 & 0,921 \\
1 & 0 & 0 & 0 & 0 & 0 & 1,034 & 1,034 & 1,034 & 1,034 \\
5 & 1 & 1 & 1 & 1 & 1 & 1,029 & 1,029 & 1,020 & 0,999 \\
0 & 1 & 0 & 0 & 0 & 0 & 1,034 & 1,034 & 1,034 & 1,034 \\
1 & 5 & 1 & 1 & 1 & 1 & 1,033 & 1,011 & 1,015 & 0,990 \\
0 & 0 & 1 & 0 & 0 & 0 & 0,887 & 0,887 & 0,887 & 0,887 \\
1 & 1 & 5 & 1 & 1 & 1 & 1,032 & 1,018 & 1,018 & 0,994 \\
0 & 0 & 0 & 1 & 0 & 0 & 1,034 & 1,034 & 1,034 & 1,034 \\
1 & 1 & 1 & 5 & 1 & 1 & 1,033 & 1,029 & 1,020 & 0,999 \\
0 & 0 & 0 & 0 & 1 & 0 & 0,740 & 0,740 & 0,740 & 0,740 \\
1 & 1 & 1 & 1 & 5 & 1 & 1,030 & 0,823 & 0,814 & 0,802 \\
0 & 0 & 0 & 0 & 0 & 1 & 1,034 & 1,034 & 1,034 & 1,034 \\
1 & 1 & 1 & 1 & 1 & 5 & 1,034 & 1,034 & 1,024 & 1,007 \\
1 & 5 & 5 & 5 & 1 & 1 & 1,033 & 1,031 & 1,031 & 0,999 \\
5 & 1 & 1 & 1 & 1 & 5 & 1,033 & 1,030 & 1,030 & 1,006 \\
\hline
\end{tabular}


Tabla A-II.7 Datos obtenidos para la variable MH (ktep).

\begin{tabular}{rrrrrrcccc}
\hline \multicolumn{1}{c}{ Pesos } & F2 & F3 & F4 & F5 & F6 & L1 & L2 & L3 & Lo \\
1 & 1 & 1 & 1 & 1 & 1 & 3,327 & 3,525 & 3,524 & 3,582 \\
1 & 0 & 0 & 0 & 0 & 0 & 3,474 & 3,474 & 3,474 & 3,474 \\
5 & 1 & 1 & 1 & 1 & 1 & 3,382 & 3,481 & 3,469 & 3,452 \\
0 & 1 & 0 & 0 & 0 & 0 & 1,687 & 1,687 & 1,687 & 1,687 \\
1 & 5 & 1 & 1 & 1 & 1 & 3,155 & 3,326 & 3,454 & 3,247 \\
0 & 0 & 1 & 0 & 0 & 0 & 1,687 & 1,687 & 1,687 & 1,687 \\
1 & 1 & 5 & 1 & 1 & 1 & 3,039 & 3,351 & 3,351 & 3,437 \\
0 & 0 & 0 & 1 & 0 & 0 & 3,725 & 3,725 & 3,725 & 3,725 \\
1 & 1 & 1 & 5 & 1 & 1 & 3,601 & 3,637 & 3,675 & 3,665 \\
0 & 0 & 0 & 0 & 1 & 0 & 3,725 & 3,725 & 3,725 & 3,725 \\
1 & 1 & 1 & 1 & 5 & 1 & 3,348 & 3,666 & 3,689 & 3,634 \\
0 & 0 & 0 & 0 & 0 & 1 & 0,976 & 0,976 & 0,976 & 0,976 \\
1 & 1 & 1 & 1 & 1 & 5 & 3,204 & 3,204 & 3,476 & 3,435 \\
1 & 5 & 5 & 5 & 1 & 1 & 3,386 & 3,591 & 3,591 & 3,601 \\
5 & 1 & 1 & 1 & 1 & 5 & 3,231 & 3,429 & 3,504 & 3,432 \\
\hline
\end{tabular}

Tabla A-II.8 Datos obtenidos para la variable GEO (ktep).

\begin{tabular}{rrrrrrcccc}
\hline \multicolumn{1}{c}{ Pesos } & & & \multicolumn{4}{c}{ VARIACIÓN DE GEO } \\
F1 & F2 & F3 & F4 & F5 & F6 & L1 & L2 & L3 & L \\
1 & 1 & 1 & 1 & 1 & 1 & 0,204 & 0,193 & 0,178 & 0,146 \\
1 & 0 & 0 & 0 & 0 & 0 & 0,205 & 0,205 & 0,205 & 0,205 \\
5 & 1 & 1 & 1 & 1 & 1 & 0,205 & 0,203 & 0,197 & 0,187 \\
0 & 1 & 0 & 0 & 0 & 0 & 0,205 & 0,205 & 0,205 & 0,205 \\
1 & 5 & 1 & 1 & 1 & 1 & 0,204 & 0,199 & 0,197 & 0,186 \\
0 & 0 & 1 & 0 & 0 & 0 & 0,205 & 0,205 & 0,205 & 0,205 \\
1 & 1 & 5 & 1 & 1 & 1 & 0,205 & 0,200 & 0,200 & 0,187 \\
0 & 0 & 0 & 1 & 0 & 0 & 0,205 & 0,205 & 0,205 & 0,205 \\
1 & 1 & 1 & 5 & 1 & 1 & 0,205 & 0,203 & 0,198 & 0,188 \\
0 & 0 & 0 & 0 & 1 & 0 & 0,050 & 0,050 & 0,050 & 0,050 \\
1 & 1 & 1 & 1 & 5 & 1 & 0,203 & 0,094 & 0,089 & 0,085 \\
0 & 0 & 0 & 0 & 0 & 1 & 0,205 & 0,205 & 0,205 & 0,205 \\
1 & 1 & 1 & 1 & 1 & 5 & 0,205 & 0,205 & 0,200 & 0,195 \\
1 & 5 & 5 & 5 & 1 & 1 & 0,205 & 0,204 & 0,204 & 0,188 \\
5 & 1 & 1 & 1 & 1 & 5 & 0,205 & 0,204 & 0,203 & 0,193 \\
\hline
\end{tabular}


Tabla A-II.9 Datos obtenidos para la función $\mathrm{F}_{1}$ (ktep).

\begin{tabular}{rrrrrrcccc}
\hline \multicolumn{1}{c}{ Pesos } & & & \multicolumn{4}{c}{ VARIACIÓN DE F1 } \\
F1 & F2 & F3 & F4 & F5 & F6 & L1 & L2 & L3 & L \\
1 & 1 & 1 & 1 & 1 & 1 & 44,345 & 42,728 & 40,598 & 35,827 \\
1 & 0 & 0 & 0 & 0 & 0 & 44,661 & 44,661 & 44,661 & 44,661 \\
5 & 1 & 1 & 1 & 1 & 1 & 44,525 & 44,324 & 43,472 & 41,896 \\
0 & 1 & 0 & 0 & 0 & 0 & 43,471 & 43,471 & 43,471 & 43,471 \\
1 & 5 & 1 & 1 & 1 & 1 & 44,268 & 43,550 & 43,379 & 41,591 \\
0 & 0 & 1 & 0 & 0 & 0 & 43,324 & 43,324 & 43,324 & 43,324 \\
1 & 1 & 5 & 1 & 1 & 1 & 44,250 & 43,780 & 43,780 & 41,843 \\
0 & 0 & 0 & 1 & 0 & 0 & 44,490 & 44,490 & 44,490 & 44,490 \\
1 & 1 & 1 & 5 & 1 & 1 & 44,474 & 44,224 & 43,479 & 41,946 \\
0 & 0 & 0 & 0 & 1 & 0 & 21,724 & 21,724 & 21,724 & 21,724 \\
1 & 1 & 1 & 1 & 5 & 1 & 44,088 & 28,174 & 27,483 & 26,816 \\
0 & 0 & 0 & 0 & 0 & 1 & 43,471 & 43,471 & 43,471 & 43,471 \\
1 & 1 & 1 & 1 & 1 & 5 & 44,349 & 44,349 & 43,744 & 42,925 \\
1 & 5 & 5 & 5 & 1 & 1 & 44,342 & 44,315 & 44,315 & 41,945 \\
5 & 1 & 1 & 1 & 1 & 5 & 44,509 & 44,486 & 44,291 & 42,848 \\
\hline
\end{tabular}

Tabla A-II.10 Datos obtenidos para la función $\mathrm{F}_{2}(\mathrm{ktep})$.

\begin{tabular}{rrrrrrcccc}
\hline \multicolumn{1}{c}{ Pesos } & \multicolumn{1}{c}{ VARIACIÓN DE F2 } \\
F1 & F2 & F3 & F4 & F5 & F6 & L1 & L2 & L3 & L \\
1 & 1 & 1 & 1 & 1 & 1 & 163,380 & 155,709 & 146,563 & 125,949 \\
1 & 0 & 0 & 0 & 0 & 0 & 163,260 & 163,260 & 163,260 & 163,260 \\
5 & 1 & 1 & 1 & 1 & 1 & 163,247 & 162,229 & 158,445 & 151,734 \\
0 & 1 & 0 & 0 & 0 & 0 & 163,931 & 163,931 & 163,931 & 163,931 \\
1 & 5 & 1 & 1 & 1 & 1 & 163,153 & 159,950 & 158,699 & 151,714 \\
0 & 0 & 1 & 0 & 0 & 0 & 163,656 & 163,656 & 163,656 & 163,656 \\
1 & 1 & 5 & 1 & 1 & 1 & 163,500 & 160,878 & 160,878 & 152,319 \\
0 & 0 & 0 & 1 & 0 & 0 & 163,865 & 163,865 & 163,865 & 163,865 \\
1 & 1 & 1 & 5 & 1 & 1 & 163,724 & 162,693 & 159,402 & 152,790 \\
0 & 0 & 0 & 0 & 1 & 0 & 64,481 & 64,481 & 64,481 & 64,481 \\
1 & 1 & 1 & 1 & 5 & 1 & 162,499 & 92,518 & 89,459 & 86,750 \\
0 & 0 & 0 & 0 & 0 & 1 & 163,220 & 163,220 & 163,220 & 163,220 \\
1 & 1 & 1 & 1 & 1 & 5 & 163,409 & 163,409 & 160,381 & 157,143 \\
1 & 5 & 5 & 5 & 1 & 1 & 163,813 & 163,019 & 163,019 & 152,898 \\
5 & 1 & 1 & 1 & 1 & 5 & 163,252 & 163,147 & 162,147 & 155,956 \\
\hline
\end{tabular}


Tabla A-II.11 Datos obtenidos para la función $\mathrm{F}_{3}$ (ktep).

\begin{tabular}{rrrrrrcccc}
\hline \multicolumn{1}{c}{ Pesos } & & & \multicolumn{4}{c}{ VARIACIÓN DE F3 } \\
F1 & F2 & F3 & F4 & F5 & F6 & L1 & L2 & L3 & L \\
1 & 1 & 1 & 1 & 1 & 1 & 0,409 & 0,390 & 0,368 & 0,319 \\
1 & 0 & 0 & 0 & 0 & 0 & 0,408 & 0,408 & 0,408 & 0,408 \\
5 & 1 & 1 & 1 & 1 & 1 & 0,408 & 0,405 & 0,396 & 0,380 \\
0 & 1 & 0 & 0 & 0 & 0 & 0,410 & 0,410 & 0,410 & 0,410 \\
1 & 5 & 1 & 1 & 1 & 1 & 0,408 & 0,400 & 0,397 & 0,381 \\
0 & 0 & 1 & 0 & 0 & 0 & 0,410 & 0,410 & 0,410 & 0,410 \\
1 & 1 & 5 & 1 & 1 & 1 & 0,409 & 0,403 & 0,403 & 0,382 \\
0 & 0 & 0 & 1 & 0 & 0 & 0,410 & 0,410 & 0,410 & 0,410 \\
1 & 1 & 1 & 5 & 1 & 1 & 0,409 & 0,407 & 0,399 & 0,383 \\
0 & 0 & 0 & 0 & 1 & 0 & 0,171 & 0,171 & 0,171 & 0,171 \\
1 & 1 & 1 & 1 & 5 & 1 & 0,406 & 0,238 & 0,231 & 0,224 \\
0 & 0 & 0 & 0 & 0 & 1 & 0,408 & 0,408 & 0,408 & 0,408 \\
1 & 1 & 1 & 1 & 1 & 5 & 0,408 & 0,408 & 0,401 & 0,394 \\
1 & 5 & 5 & 5 & 1 & 1 & 0,410 & 0,408 & 0,408 & 0,383 \\
5 & 1 & 1 & 1 & 1 & 5 & 0,408 & 0,408 & 0,405 & 0,390 \\
\hline
\end{tabular}

Tabla A-II.12 Datos obtenidos para la función $\mathrm{F}_{4}$ (ktep).

\begin{tabular}{rrrrrrcccc}
\hline \multicolumn{1}{c}{ Pesos } & \multicolumn{1}{c}{ VARIACIÓN DE F4 } \\
1 & 1 & 1 & 1 & 1 & 1 & 0,686 & 0,654 & 0,616 & 0,529 \\
1 & 0 & 0 & 0 & 0 & 0 & 0,686 & 0,686 & 0,686 & 0,686 \\
5 & 1 & 1 & 1 & 1 & 1 & 0,686 & 0,682 & 0,666 & 0,638 \\
0 & 1 & 0 & 0 & 0 & 0 & 0,688 & 0,688 & 0,688 & 0,688 \\
1 & 5 & 1 & 1 & 1 & 1 & 0,685 & 0,672 & 0,667 & 0,637 \\
0 & 0 & 1 & 0 & 0 & 0 & 0,687 & 0,687 & 0,687 & 0,687 \\
1 & 1 & 5 & 1 & 1 & 1 & 0,687 & 0,676 & 0,676 & 0,640 \\
0 & 0 & 0 & 1 & 0 & 0 & 0,688 & 0,688 & 0,688 & 0,688 \\
1 & 1 & 1 & 5 & 1 & 1 & 0,687 & 0,683 & 0,669 & 0,642 \\
0 & 0 & 0 & 0 & 1 & 0 & 0,272 & 0,272 & 0,272 & 0,272 \\
1 & 1 & 1 & 1 & 5 & 1 & 0,682 & 0,390 & 0,377 & 0,365 \\
0 & 0 & 0 & 0 & 0 & 1 & 0,686 & 0,686 & 0,686 & 0,686 \\
1 & 1 & 1 & 1 & 1 & 5 & 0,686 & 0,686 & 0,674 & 0,660 \\
1 & 5 & 5 & 5 & 1 & 1 & 0,688 & 0,685 & 0,685 & 0,642 \\
5 & 1 & 1 & 1 & 1 & 5 & 0,686 & 0,685 & 0,681 & 0,655 \\
\hline
\end{tabular}


Tabla A-II.13 Datos obtenidos para la función $\mathrm{F}_{5}(\mathrm{ktep})$.

\begin{tabular}{rrrrrrcccc}
\hline \multicolumn{1}{c}{ Pesos } & \multicolumn{1}{c}{ VARIACIÓN DE F5 } \\
F1 & F2 & F3 & F4 & F5 & F6 & L1 & L2 & L3 & Lo \\
1 & 1 & 1 & 1 & 1 & 1 & 29,996 & 28,554 & 27,396 & 24,628 \\
1 & 0 & 0 & 0 & 0 & 0 & 29,713 & 29,713 & 29,713 & 29,713 \\
5 & 1 & 1 & 1 & 1 & 1 & 29,909 & 29,528 & 29,090 & 28,278 \\
0 & 1 & 0 & 0 & 0 & 0 & 34,152 & 34,152 & 34,152 & 34,152 \\
1 & 5 & 1 & 1 & 1 & 1 & 30,432 & 29,569 & 29,110 & 28,721 \\
0 & 0 & 1 & 0 & 0 & 0 & 33,993 & 33,993 & 33,993 & 33,993 \\
1 & 1 & 5 & 1 & 1 & 1 & 30,756 & 29,626 & 29,626 & 28,327 \\
0 & 0 & 0 & 1 & 0 & 0 & 29,009 & 29,009 & 29,009 & 29,009 \\
1 & 1 & 1 & 5 & 1 & 1 & 29,330 & 29,100 & 28,586 & 27,775 \\
0 & 0 & 0 & 0 & 1 & 0 & 16,500 & 16,500 & 16,500 & 16,500 \\
1 & 1 & 1 & 1 & 5 & 1 & 29,822 & 20,196 & 19,753 & 19,542 \\
0 & 0 & 0 & 0 & 0 & 1 & 36,030 & 36,030 & 36,030 & 36,030 \\
1 & 1 & 1 & 1 & 1 & 5 & 30,342 & 30,342 & 29,257 & 28,931 \\
1 & 5 & 5 & 5 & 1 & 1 & 29,869 & 29,270 & 29,270 & 27,951 \\
5 & 1 & 1 & 1 & 1 & 5 & 30,313 & 29,767 & 29,452 & 28,854 \\
\hline
\end{tabular}

Tabla A-II.14 Datos obtenidos para la función $\mathrm{F}_{6}$ (ktep).

\begin{tabular}{rrrrrrcccc}
\hline \multicolumn{1}{c}{ Pesos } & \multicolumn{1}{c}{ VARIACIÓN DE F6 } \\
F1 & F2 & F3 & F4 & F5 & F6 & L1 & L2 & L3 & Lo \\
1 & 1 & 1 & 1 & 1 & 1 & 40,813 & 39,011 & 36,896 & 32,099 \\
1 & 0 & 0 & 0 & 0 & 0 & 40,982 & 40,982 & 40,982 & 40,982 \\
5 & 1 & 1 & 1 & 1 & 1 & 40,938 & 40,640 & 39,806 & 38,257 \\
0 & 1 & 0 & 0 & 0 & 0 & 41,579 & 41,579 & 41,579 & 41,579 \\
1 & 5 & 1 & 1 & 1 & 1 & 40,909 & 40,026 & 39,729 & 38,158 \\
0 & 0 & 1 & 0 & 0 & 0 & 41,432 & 41,432 & 41,432 & 41,432 \\
1 & 1 & 5 & 1 & 1 & 1 & 41,006 & 40,228 & 40,228 & 38,219 \\
0 & 0 & 0 & 1 & 0 & 0 & 40,560 & 40,560 & 40,560 & 40,560 \\
1 & 1 & 1 & 5 & 1 & 1 & 40,668 & 40,385 & 39,607 & 38,094 \\
0 & 0 & 0 & 0 & 1 & 0 & 17,948 & 17,948 & 17,948 & 17,948 \\
1 & 1 & 1 & 1 & 5 & 1 & 40,537 & 24,413 & 23,705 & 23,097 \\
0 & 0 & 0 & 0 & 0 & 1 & 42,290 & 42,290 & 42,290 & 42,290 \\
1 & 1 & 1 & 1 & 1 & 5 & 40,941 & 40,941 & 40,068 & 39,296 \\
1 & 5 & 5 & 5 & 1 & 1 & 40,751 & 40,521 & 40,521 & 38,156 \\
5 & 1 & 1 & 1 & 1 & 5 & 41,073 & 40,853 & 40,584 & 39,223 \\
\hline
\end{tabular}


ANEXO III

DATOS OBTENIDOS DEL ANÁLISIS DE SENSIBILIDAD 
Tabla A-III.1 Datos obtenidos del Análisis de Sensibilidad (AdS) para LEÑ con incremento del precio de petróleo en $50 \%$ (ktep).

\begin{tabular}{cccccccc}
\hline \multicolumn{9}{c}{ Pesos } & \multicolumn{3}{c}{ VARIACIÓN DE LEÑ } \\
F1 & F2 & F3 & F4 & F5 & F6 & Inicial & AdS \\
1 & 1 & 1 & 1 & 1 & 1 & 8,514 & 8,355 \\
1 & 0 & 0 & 0 & 0 & 0 & 12,693 & 12,693 \\
5 & 1 & 1 & 1 & 1 & 1 & 11,401 & 11,410 \\
0 & 1 & 0 & 0 & 0 & 0 & 12,693 & 12,693 \\
1 & 5 & 1 & 1 & 1 & 1 & 11,370 & 11,403 \\
0 & 0 & 1 & 0 & 0 & 0 & 12,693 & 12,693 \\
1 & 1 & 5 & 1 & 1 & 1 & 11,440 & 11,385 \\
0 & 0 & 0 & 1 & 0 & 0 & 12,693 & 12,693 \\
1 & 1 & 1 & 5 & 1 & 1 & 11,473 & 11,422 \\
0 & 0 & 0 & 0 & 1 & 0 & 1,700 & 1,700 \\
1 & 1 & 1 & 1 & 5 & 1 & 4,170 & 4,089 \\
0 & 0 & 0 & 0 & 0 & 1 & 12,693 & 12,693 \\
1 & 1 & 1 & 1 & 1 & 5 & 11,970 & 11,866 \\
1 & 5 & 5 & 5 & 1 & 1 & 11,485 & 11,409 \\
5 & 1 & 1 & 1 & 1 & 5 & 11,869 & 11,869 \\
\hline
\end{tabular}

Tabla A-III.2 Datos obtenidos del Análisis de Sensibilidad (AdS) para CV con incremento del precio de petróleo en $50 \%$ (ktep).

\begin{tabular}{cccccccc}
\hline \multicolumn{9}{c}{ Pesos } & \multicolumn{3}{c}{ VARIACIÓN DE CV } \\
F1 & F2 & F3 & F4 & F5 & F6 & Inicial & AdS \\
1 & 1 & 1 & 1 & 1 & 1 & 2,057 & 2,032 \\
1 & 0 & 0 & 0 & 0 & 0 & 2,705 & 2,705 \\
5 & 1 & 1 & 1 & 1 & 1 & 2,504 & 2,506 \\
0 & 1 & 0 & 0 & 0 & 0 & 2,705 & 2,705 \\
1 & 5 & 1 & 1 & 1 & 1 & 2,500 & 2,505 \\
0 & 0 & 1 & 0 & 0 & 0 & 2,705 & 2,705 \\
1 & 1 & 5 & 1 & 1 & 1 & 2,510 & 2,502 \\
0 & 0 & 0 & 1 & 0 & 0 & 2,705 & 2,705 \\
1 & 1 & 1 & 5 & 1 & 1 & 2,515 & 2,508 \\
0 & 0 & 0 & 0 & 1 & 0 & 1,000 & 1,000 \\
1 & 1 & 1 & 1 & 5 & 1 & 1,383 & 1,370 \\
0 & 0 & 0 & 0 & 0 & 1 & 2,705 & 2,705 \\
1 & 1 & 1 & 1 & 1 & 5 & 2,593 & 2,576 \\
1 & 5 & 5 & 5 & 1 & 1 & 2,517 & 2,506 \\
5 & 1 & 1 & 1 & 1 & 5 & 2,577 & 2,577 \\
\hline
\end{tabular}


Tabla A-III.3 Datos obtenidos del Análisis de Sensibilidad (AdS) para BL con incremento del precio de petróleo en $50 \%$ (ktep).

\begin{tabular}{cccccccc}
\hline \multicolumn{9}{c}{ Pesos } & \multicolumn{3}{c}{ VARIACIÓN DE BL } \\
F1 & F2 & F3 & F4 & F5 & F6 & Inicial & AdS \\
1 & 1 & 1 & 1 & 1 & 1 & 0,921 & 0,918 \\
1 & 0 & 0 & 0 & 0 & 0 & 1,034 & 1,034 \\
5 & 1 & 1 & 1 & 1 & 1 & 0,999 & 0,992 \\
0 & 1 & 0 & 0 & 0 & 0 & 1,034 & 1,034 \\
1 & 5 & 1 & 1 & 1 & 1 & 0,990 & 0,985 \\
0 & 0 & 1 & 0 & 0 & 0 & 0,887 & 0,887 \\
1 & 1 & 5 & 1 & 1 & 1 & 0,994 & 0,997 \\
0 & 0 & 0 & 1 & 0 & 0 & 1,034 & 1,034 \\
1 & 1 & 1 & 5 & 1 & 1 & 0,999 & 1,000 \\
0 & 0 & 0 & 0 & 1 & 0 & 0,740 & 0,740 \\
1 & 1 & 1 & 1 & 5 & 1 & 0,802 & 0,801 \\
0 & 0 & 0 & 0 & 0 & 1 & 1,034 & 1,034 \\
1 & 1 & 1 & 1 & 1 & 5 & 1,007 & 1,011 \\
1 & 5 & 5 & 5 & 1 & 1 & 0,999 & 0,998 \\
5 & 1 & 1 & 1 & 1 & 5 & 1,006 & 1,010 \\
\hline
\end{tabular}

Tabla A-III.3 Datos obtenidos del Análisis de Sensibilidad (AdS) para las alternativas energéticas estudiadas con incremento del precio de petróleo en 50\%. Distancia L $\infty$ (ktep).

\begin{tabular}{cccccccccccccc}
\hline \multicolumn{1}{c}{ Pesos } & & & ST & PV & EOL & LEN & CV & BL & MH & GEO \\
\hline $\mathbf{F}_{\mathbf{1}}$ & $\mathbf{F}_{\mathbf{2}}$ & $\mathbf{F}_{\mathbf{3}}$ & $\mathbf{F}_{\mathbf{4}}$ & $\mathbf{F}_{\mathbf{5}}$ & $\mathbf{F}_{\mathbf{6}}$ & & & & & & & \\
\hline 1 & 1 & 1 & 1 & 1 & 1 & 1,043 & 2,790 & 0,402 & 8,355 & 2,032 & 0,918 & 3,532 & 0,144 \\
1 & 0 & 0 & 0 & 0 & 0 & 1,658 & 3,238 & 0,013 & 12,693 & 2,705 & 1,034 & 3,474 & 0,205 \\
5 & 1 & 1 & 1 & 1 & 1 & 1,476 & 2,888 & 0,340 & 11,410 & 2,506 & 0,992 & 3,496 & 0,187 \\
0 & 1 & 0 & 0 & 0 & 0 & 1,658 & 2,644 & 2,393 & 12,693 & 2,705 & 1,034 & 1,687 & 0,205 \\
1 & 5 & 1 & 1 & 1 & 1 & 1,475 & 2,659 & 0,661 & 11,403 & 2,505 & 0,985 & 3,404 & 0,187 \\
0 & 0 & 1 & 0 & 0 & 0 & 1,658 & 2,644 & 2,393 & 12,693 & 2,705 & 0,887 & 1,687 & 0,205 \\
1 & 1 & 5 & 1 & 1 & 1 & 1,472 & 2,926 & 0,494 & 11,385 & 2,502 & 0,997 & 3,304 & 0,186 \\
0 & 0 & 0 & 1 & 0 & 0 & 1,658 & 2,644 & 0,355 & 12,693 & 2,705 & 1,034 & 3,725 & 0,205 \\
1 & 1 & 1 & 5 & 1 & 1 & 1,478 & 2,741 & 0,341 & 11,422 & 2,508 & 1,000 & 3,642 & 0,187 \\
0 & 0 & 0 & 0 & 1 & 0 & 0,100 & 2,644 & 0,355 & 1,700 & 1,000 & 0,740 & 3,725 & 0,050 \\
1 & 1 & 1 & 1 & 5 & 1 & 0,439 & 2,732 & 0,380 & 4,089 & 1,370 & 0,801 & 3,612 & 0,084 \\
0 & 0 & 0 & 0 & 0 & 1 & 1,658 & 3,355 & 2,393 & 12,693 & 2,705 & 1,034 & 0,976 & 0,205 \\
1 & 1 & 1 & 1 & 1 & 5 & 1,540 & 2,947 & 0,433 & 11,866 & 2,576 & 1,011 & 3,345 & 0,193 \\
1 & 5 & 5 & 5 & 1 & 1 & 1,476 & 2,734 & 0,396 & 11,409 & 2,506 & 0,998 & 3,595 & 0,187 \\
5 & 1 & 1 & 1 & 1 & 5 & 1,541 & 3,078 & 0,204 & 11,869 & 2,577 & 1,010 & 3,442 & 0,193 \\
\hline
\end{tabular}


Tabla A-III.4 Datos obtenidos del Análisis de Sensibilidad (AdS) para las alternativas energéticas estudiadas con disminución del PIB a 3,5\%. Distancia L $\infty$ (ktep).

\begin{tabular}{cccccccccccccc}
\hline \multicolumn{1}{c}{ Pesos } & & & ST & PV & EOL & LEN & CV & BL & MH & GEO \\
\hline $\mathbf{F}_{\mathbf{1}}$ & $\mathbf{F}_{\mathbf{2}}$ & $\mathbf{F}_{\mathbf{3}}$ & $\mathbf{F}_{\mathbf{4}}$ & $\mathbf{F}_{\mathbf{5}}$ & $\mathbf{F}_{\mathbf{6}}$ & & & & & & & \\
1 & 1 & 1 & 1 & 1 & 1 & 0,922 & 2,342 & 0,102 & 7,387 & 1,795 & 0,761 & 3,069 & 0,127 \\
1 & 0 & 0 & 0 & 0 & 0 & 1,359 & 2,696 & 0,013 & 10,409 & 2,218 & 0,848 & 2,804 & 0,168 \\
5 & 1 & 1 & 1 & 1 & 1 & 1,223 & 2,547 & 0,064 & 9,465 & 2,086 & 0,820 & 2,902 & 0,155 \\
0 & 1 & 0 & 0 & 0 & 0 & 1,359 & 2,178 & 2,393 & 10,409 & 2,218 & 0,848 & 0,942 & 0,168 \\
1 & 5 & 1 & 1 & 1 & 1 & 1,232 & 2,350 & 0,175 & 9,528 & 2,095 & 0,818 & 2,988 & 0,156 \\
0 & 0 & 1 & 0 & 0 & 0 & 1,359 & 2,178 & 2,393 & 10,409 & 2,218 & 0,723 & 0,942 & 0,168 \\
1 & 1 & 5 & 1 & 1 & 1 & 1,242 & 2,385 & 0,248 & 9,598 & 2,105 & 0,817 & 2,881 & 0,157 \\
0 & 0 & 0 & 1 & 0 & 0 & 1,359 & 2,178 & 0,013 & 10,409 & 2,218 & 0,848 & 3,322 & 0,168 \\
1 & 1 & 1 & 5 & 1 & 1 & 1,231 & 2,219 & 0,055 & 9,523 & 2,094 & 0,822 & 3,239 & 0,156 \\
0 & 0 & 0 & 0 & 1 & 0 & 0,100 & 2,178 & 0,013 & 1,700 & 1,000 & 0,598 & 3,322 & 0,050 \\
1 & 1 & 1 & 1 & 5 & 1 & 0,402 & 2,226 & 0,061 & 3,787 & 1,292 & 0,656 & 3,227 & 0,078 \\
0 & 0 & 0 & 0 & 0 & 1 & 1,359 & 3,355 & 1,658 & 10,409 & 2,218 & 0,848 & 0,500 & 0,168 \\
1 & 1 & 1 & 1 & 1 & 5 & 1,317 & 2,474 & 0,238 & 10,119 & 2,177 & 0,839 & 2,802 & 0,164 \\
1 & 5 & 5 & 5 & 1 & 1 & 1,232 & 2,270 & 0,088 & 9,529 & 2,095 & 0,819 & 3,155 & 0,156 \\
5 & 1 & 1 & 1 & 1 & 5 & 1,294 & 2,628 & 0,062 & 9,956 & 2,155 & 0,835 & 2,823 & 0,162 \\
\hline
\end{tabular}




\section{ANEXO IV \\ PAPER PUBLICADO EN LA REVISTA "JOURNAL OF RENEWABLE AND SUSTAINABLE ENERGY"}




\section{Energy planning model with renewable energy using optimization multicriteria techniques for isolated rural communities: Cajamarca province, Peru}

Eder Jesús Falcón-Roque, Francisco Marcos Martín, Cristina Pascual Castaño, Luis Carlos DomínguezDafauce, and Francisco Javier Bastante Flores

Citation: Journal of Renewable and Sustainable Energy 9, 065903 (2017);

View online: https://doi.org/10.1063/1.4989574

View Table of Contents: http://aip.scitation.org/toc/rse/9/6

Published by the American Institute of Physics 


\title{
Energy planning model with renewable energy using optimization multicriteria techniques for isolated rural communities: Cajamarca province, Peru
}

\author{
Eder Jesús Falcón-Roque, ${ }^{1, a)}$ Francisco Marcos Martín, ${ }^{1}$ \\ Cristina Pascual Castaño, ${ }^{1}$ Luis Carlos Domínguez-Dafauce, ${ }^{1}$ \\ and Francisco Javier Bastante Flores ${ }^{2}$ \\ ${ }^{1}$ Escuela Técnica Superior de Ingenieros de Montes, Universidad Politécnica de Madrid, \\ Madrid, Spain \\ ${ }^{2}$ Departamento de Tecnología Química, Escuela Técnica Superior de Ingenieros \\ Industriales, Universidad Politécnica de Madrid, Madrid, Spain
}

(Received 9 June 2017; accepted 5 October 2017; published online 16 November 2017)

\begin{abstract}
One of the seventeen Sustainable Development Goals (SDGs) introduced by the United Nations is SDG 7, Affordable and Clean Energy: "to ensure access to affordable, reliable and modern energy for all." Peru is one of the Latin America and Caribbean countries that has signed this commitment. The Peruvian government encourages the supply of electricity from renewable energy sources to isolated rural communities, and renewable energy is considered in the country energy planning. This paper presents an energy planning model based on multicriteria optimization techniques, named SEPLAN (Sustainable Energy Planning). The SEPLAN model allows the incorporation of objectives that could be in conflict: for example, economic, environmental, or social objectives, as well as universal access to energy. In this model, six objective functions were established: replaceable amounts of fossil energy; $\mathrm{CO}_{2}$, $\mathrm{NO}_{\mathrm{X}}$, and $\mathrm{SO}_{2}$ avoidable emissions; cost minimization; and universal access to energy in isolated rural communities. Linear programming was applied to the six objective functions, and optimal solutions were chosen for each one. The efficient solutions were found with compromise programming, and Chebysev distance $\left(L_{\infty}\right)$ was chosen as the Pareto optimal solution. The proposed model was applied in Cajamarca province, Peru. Cajamarca has the lowest electrification rate of the country due to the very high dispersion of households of rural communities. The results for the analysed scenarios provided the prioritization of the energy alternatives considered. When encouraging the objective function related to universal access to energy in isolated rural communities, photovoltaic solar energy was the priority in power energy generation. Thereby, the model could become a useful tool for decision makers. Published by AIP Publishing. https://doi.org/10.1063/1.4989574
\end{abstract}

\section{INTRODUCTION}

Today, approximately 1.2 billion people (17\% of the global population) live without electricity, and 2.7 billion people ( $38 \%$ of the global population) put their health at risk by using firewood for cooking. ${ }^{1}$

Of the countries in Latin America and the Caribbean (LAC), Peru is experiencing a difficult situation in terms of access to electricity that does not correspond to its degree of development. The Peruvian economy has expanded from USD53 billion in 2000 to USD197 billion in 2012 , becoming the 48th largest economy in the world. The high rates of economic growth

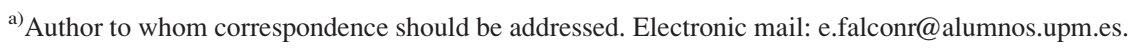


have led to a substantial increase in energy demand. However, Peru has the second highest proportion of population without access to electricity on the continent (after Haiti). As per official information, in Peru, there are 2.5 million people without access to electricity and 2.5 million households using traditional fuels. ${ }^{2}$

The electric global coverage rate in Peru is relatively high. Nevertheless, the access to electricity in rural areas is still a concern. This is the case of the Cajamarca region, located in the north of Peru, where $70 \%$ of the population lives in rural areas. ${ }^{3}$ On the other hand, the dispersion of households in the rural areas has caused the index of electrification in the Cajamarca region to be the lowest in the country. ${ }^{4}$

Such situations encourage many energy planners to develop optimization models that take into account vulnerability (energy protection security), environmental impact, sustainability problems, and limited resources. Thus, multiobjective optimization of energy systems has become a current major field of study. ${ }^{5}$

Energy planning using multicriteria analysis has attracted the attention of decision makers for a long time. ${ }^{6}$ The planning endeavour involves finding a set of sources and conversion devices so as to meet the energy requirements/demands of all the tasks optimally. ${ }^{7}$ In order to achieve this, energy planning models are widely used for evaluating and selecting among different energy technologies.

Currently, the efforts in LAC are focused on supplying energy to rural areas with new models of provision of energy services. These models should ensure sustainability and affordability to low-income populations. ${ }^{8}$ Additionally, the use of renewable energy (RE) is becoming an economically competitive and technically reliable alternative, especially in rural areas, because (i) REs are native sources, so they reduce energy dependence; (ii) REs promote local development; and (iii) REs are respectful to the environment. ${ }^{2}$

In recent years, several energy planning models for rural areas that incorporate REs have been developed. Ferrer-Martí et al. ${ }^{9}$ proposed the mixed integer linear programming (MILP) model in order to optimize the design of hybrid wind-photovoltaic (PV) systems in an isolated community. On the other hand, software such as HOMER (Hybrid Optimization Model for Multiple Energy Resources), ViPOR, or similar have systematized the design and sizing of planning projects. This kind of software only incorporates quantitative analysis in a project scheme and is used as a tool in planning. However, according to Nerini et al. ${ }^{10}$ appropriate energy planning in rural areas requires a broader overview and should consider technical, economic, environmental, social, and institutional criteria in a multicriteria approach. Most times, the inclusion of environmental and social criteria involves using qualitative approaches (e.g., the precedence method ${ }^{12,15,16}$ ) to assign weights according to interviews with experts in order to rank the criteria, e.g., Nerini et al ${ }^{10}$ in Brazilian Amazon; Amer and Daim ${ }^{11}$ in Pakistan; or Domínguez and Marcos ${ }^{12}$ in Sri Lanka.

Rojas-Zerpa and Yusta, ${ }^{13}$ in their review of methodologies and applications for electric supply in rural remote areas, found that only $2 \%$ of the studies were carried out in Peru. Those authors also concluded that in rural remote areas, coherent and robust plans may take into consideration the needs of the local population. Similarly, some nongovernmental organizations (NGOs) consider that in isolated rural communities (IRCs), technological solutions are not enough to ensure the sustainability of energy planning. According to the social microutility company ACCIONA Microenergía Perú (AMP), the social and cultural aspects of the local population are key issues to bear in mind for decision-making models in these areas. However, very few models take into account these criteria, as knowing the needs of local population is difficult.

Within this context, the objective of the present study is to develop an original energy planning model for isolated rural communities (IRCs) using optimization multicriteria tools. This model, named SEPLAN (Sustainable Energy Planning), includes an innovative function to evaluate the viability of the different RE technologies for a sustainable supply of energy in IRC. The SEPLAN model also contains other objective functions (OFs): (i) replaceable amounts of fossil energy; (ii) avoidance of greenhouse gas emissions; and (iii) the cost function, 
which were obtained based on the sustainable and renewable implementation multicriteria energy (SRIME) model. ${ }^{12}$

\section{SEPLAN MODEL}

The SEPLAN model has been built using mathematical techniques of multicriteria decision theory combined with vector analysis techniques. Figure 1 shows the general outline carried out to construct the model.

The first step was to perform a comprehensive review of current energy consumption in different production sectors. Then, the forecast demand for the planning horizon was obtained (year 2025) (Fig. 1). This horizon is the same as the one defined in the National Energy Plan published in 2014, ${ }^{14}$ which also contains Gross Domestic Product (GDP) growth assumptions for its forecast $(4.0 \%$ and $6.5 \%)$. In order to take into account the national energy policies, the authors considered the same assumptions of the mentioned plan. At this stage, information was collected from official sources on the potential supply of renewable energy in the study area.

The meetings of the decision makers and experts led the study towards an appropriate use of resources. This stage allowed prioritization of the most appropriate technologies for Cajamarca province, according to local reality and experts experience.

The next step was setting up the constraints and the definition of energy alternatives. Then, the objective functions (OFs) were built (Fig. 1). In order to facilitate the resolution of the decisional problem, linear functions were considered. Once OFs were defined, the independent optimization of each function was established using linear programming, and ideal and anti-ideal values were obtained. Finally, the set of optimal solutions was obtained by minimizing the distances to the ideal point $\left(L_{1}, L_{\infty}\right.$, and $\left.L_{k}\right)$. These distances were obtained using $\mathrm{Matlab}^{\circledR}$ software.

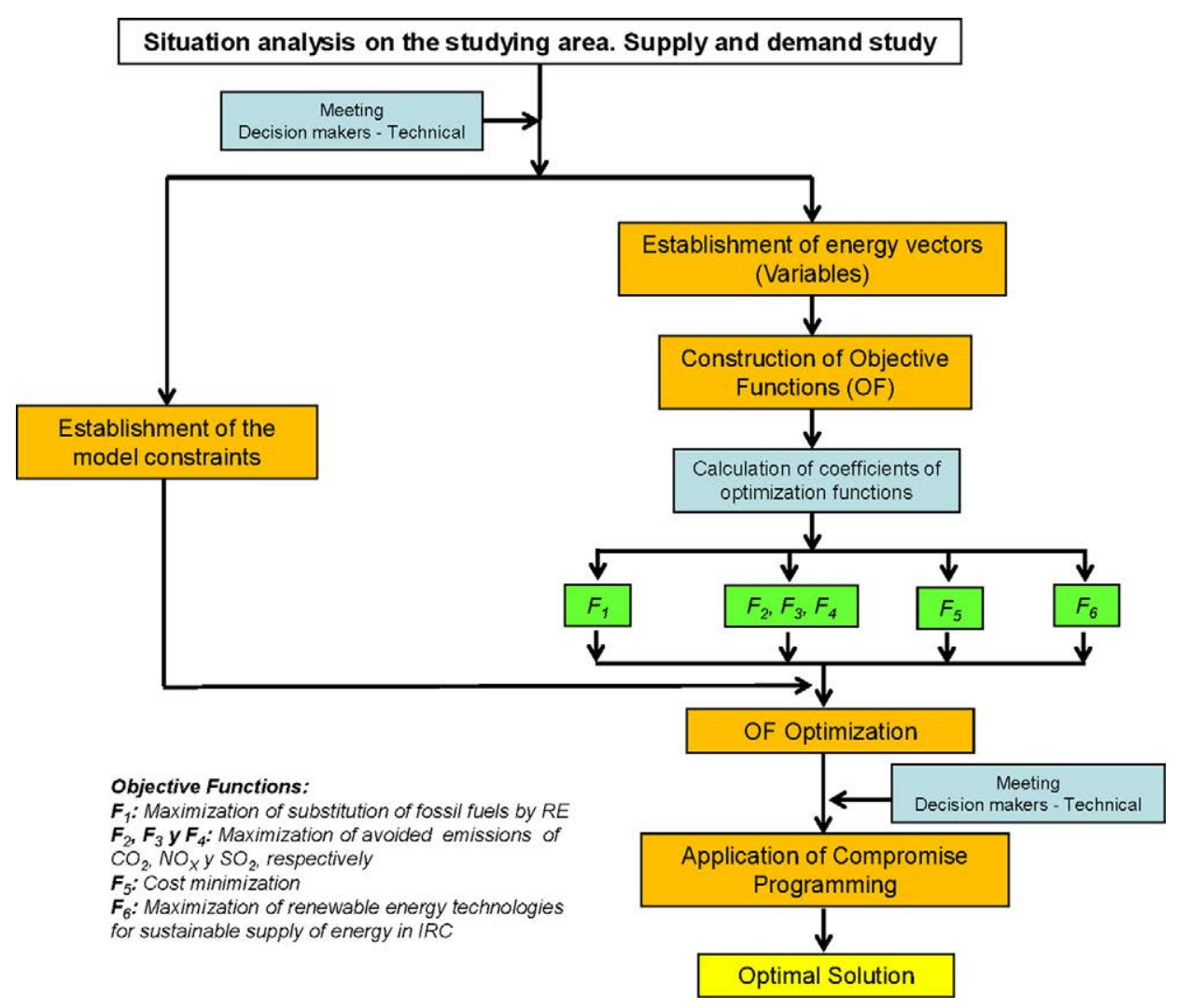

FIG. 1. SEPLAN model. 
TABLE I. Energy alternatives in Cajamarca province.

\begin{tabular}{|c|c|c|}
\hline Energy alternatives & Variable symbol & Description \\
\hline \multirow[t]{2}{*}{ Solar photovoltaic } & PV & $\begin{array}{l}\text { Solar home systems with a capacity of up to } 320 \mathrm{~W} \text {; generally, PV } \\
\text { generator, battery, charge regulator, and three lamps }\end{array}$ \\
\hline & & Solar power plants with a capacity of up to $2 \mathrm{MW}$ \\
\hline Solar thermal & ST & Solar energy of low temperature $\left(\mathrm{T}<90^{\circ} \mathrm{C}\right)$ for home use \\
\hline Wind energy low potency & WE & $\begin{array}{l}\text { Wind turbines with capacity of } 100 \text { and } 500 \mathrm{~W} \text { for home and com- } \\
\text { munity centre use, respectively }\end{array}$ \\
\hline Firewood & FW & The use of wood from native trees and forest residues for domestic use \\
\hline Charcoal & $\mathrm{CH}$ & The use of processed wood for domestic and industrial use \\
\hline Liquid biofuels & LB & The use of gasohol and biodiesel of native origin \\
\hline Small hydro & $\mathrm{SH}$ & Small hydroelectric plants of $100-1000 \mathrm{~kW}$, installed locally \\
\hline Geothermal & GEO & $\begin{array}{c}\text { Due to the availability of natural hot springs, the direct use of this } \\
\text { energy at the household level }\end{array}$ \\
\hline
\end{tabular}

\section{A. Determination of energy alternatives}

Given that the technological applications of renewable energy are developing rapidly, multiple alternatives could be chosen. However, it is clear that a study such as this would make the planning unfeasible. ${ }^{15}$ Therefore, this study has been taken into account the different types of renewable energy that are currently being used and are potentially usable in the study area. These alternatives are presented in Table I.

\section{B. Objective functions}

The six functions to be optimized are described below. Taking into account that subscript $i$ denotes every RE type, and $j$ denotes the different sectors, $X_{i j}$ denotes the amount of fossil fuel (toe) replaced by RE $i$ in sector $j$.

\section{$F_{1}:$ Maximization of renewable energies}

The linear function has the following structure:

$$
F_{1}\left(x_{11}, x_{12}, \ldots, x_{i j}, \ldots, x_{n m}\right)=A_{11} x_{11}+A_{12} x_{12}+\cdots+A_{n m} x_{n m},
$$

where $A_{11}, A_{12}, \ldots A_{n m}$ are nondimensional coefficients.

Coefficients A were obtained using a vector analysis technique called the precedence method. ${ }^{12,15,16}$ According to this method, comparison levels were established in order to assess each energy alternative and to compare them with one another. As per this, the priority alternatives were obtained. Table II shows the comparison levels considered, a brief description of them, and the numerical evaluation performed.

The assessment of alternatives was carried out by the authors. Values from 1 to 5 were assigned for each alternative, according to the comparison levels, obtaining for each a vector of two components $\left(N_{i}\right.$ and $\left.M_{i}\right)$, where

$$
\begin{gathered}
N_{i j}=\prod_{i=1}^{n} a_{i j}, \\
M_{i j}=\prod_{i=1}^{n}\left(6-a_{i j}\right) .
\end{gathered}
$$

Once the alternatives according to the values of $N_{i}$ and $M_{i}$ were ordered, they were divided into four groups. The next values were assigned to each, ordered from major to minor importance: Group 1: value of coefficient $A_{i}=2.5$; Group 2: value of coefficient $A_{i}=2.0$; Group 3: value of coefficient $A_{i}=1.5$; and Group 4: value of coefficient $A_{i}=1.0$. These coefficients were used in the objective function. 
TABLE II. Precedence constraints of objective function $F_{1}$, brief description, and numerical evaluation (prepared by the authors based upon García ${ }^{15}$ ).

\begin{tabular}{|c|c|c|}
\hline Level and criteria & Description & Evaluation \\
\hline \multicolumn{3}{|l|}{ Technologic } \\
\hline Technological demand & Degree of technological demand by the population & $\begin{array}{l}\text { The experience gained by local suppliers shows the impact of technologies on the } \\
\text { population. The scale used ranges from } 5 \text { (high technological demand) to } 1 \text { (low tech- } \\
\text { nological demand). }\end{array}$ \\
\hline Potential investigation & Possibility of local investigation in the technology & $\begin{array}{l}\text { Research lines at local universities or research centers define the future development } \\
\text { of the technology. The scale used ranges from } 5 \text { (high potential investigation) to } 1 \\
\text { (low potential investigation) }\end{array}$ \\
\hline Know-how up-to-date & $\begin{array}{l}\text { Installations, existing applications, and possibility of their expansion to } \\
\text { increase the know-how of the technologies }\end{array}$ & $\begin{array}{c}\text { Number of projects and installations realized related to the technology. The scale } \\
\text { used ranges from } 1 \text { (low know-how) to } 5 \text { (high know-how). }\end{array}$ \\
\hline Technology improvement & Possibility of local manufacture of equipment & $\begin{array}{l}\text { The scale used ranges from } 1 \text { (low possibility of technology improvement) to } 5 \text { (high } \\
\text { possibility of technology improvement). }\end{array}$ \\
\hline Technological level achieved & Current knowledge for the application of the technology alternative & $\begin{array}{l}\text { The scale used ranges from } 5 \text { (high technological level achieved) to } 1 \text { (low technolog- } \\
\text { ical level achieved). }\end{array}$ \\
\hline \multicolumn{3}{|l|}{ Application } \\
\hline Operating time & Time that has to elapse for starting up a technological alternative & The scale used ranges from 5 (low operating time) to 1 (high operating time). \\
\hline Implementation issues & Ease or difficulty when performing an installation & The scale used ranges from 1 (difficult implementation) to 5 (easy implementation). \\
\hline Integration possibility & Ease of an alternative to operate with existing equipment & $\begin{array}{l}\text { The scale used ranges from } 5 \text { (high integration possibility) to } 1 \text { (low integration } \\
\text { possibility). }\end{array}$ \\
\hline Potential demand & Existence of a potential market that demands and consumes that alternative & The scale used ranges from 5 (high potential demand) to 1 (low potential demand). \\
\hline Supply availability & $\begin{array}{l}\text { Existence of companies willing to carry out the commercialization of supply } \\
\text { in the energy market }\end{array}$ & The scale used ranges from 1 (low supply availability) to 5 (high supply availability). \\
\hline \multicolumn{3}{|l|}{ Energy planning } \\
\hline Political interest & $\begin{array}{l}\text { Priority in the energy economy of the country; government agencies propose } \\
\text { the alternative }\end{array}$ & The scale used ranges from 5 (high political interest) to 1 (low political interest). \\
\hline Current price & Price of renewable technology compared with conventional energies & The scale used ranges from 1 (high current price) to 5 (low current price). \\
\hline Social demand & Interest of social agents in the use of the alternative & The scale used ranges from 5 (high social demand) to 1 (low social demand). \\
\hline Legislative forecast & Legal aspects that prevent or encourage the development of the alternative & $\begin{array}{l}\text { The scale used ranges from } 1 \text { (negative legislative forecast) to } 5 \text { (positive legislative } \\
\text { forecast). }\end{array}$ \\
\hline \multicolumn{3}{|l|}{ Environmental } \\
\hline Fossil fuel environmental impact & General evaluation of the environmental impact of the alternative & $\begin{array}{c}\text { The scale used ranges from } 5 \text { (low environmental impact) to } 1 \text { (high environmental } \\
\text { impact). }\end{array}$ \\
\hline
\end{tabular}




\section{$\mathrm{F}_{2}, \mathrm{~F}_{3}, \mathrm{~F}_{4}$ : Maximization of avoided emissions of $\mathrm{CO}_{2}, \mathrm{NO}_{\mathrm{X}}$, and $\mathrm{SO}_{2}$}

Functions have the following structure:

$$
\begin{aligned}
& F_{2}\left(x_{11}, x_{12}, \ldots, x_{i j}, \ldots, x_{n m}\right)=B_{11} x_{11}+B_{12} x_{12}+\cdots+B_{n m} x_{n m}, \\
& F_{3}\left(x_{11}, x_{12}, \ldots, x_{i j}, \ldots, x_{n m}\right)=C_{11} x_{11}+C_{12} x_{12}+\cdots+C_{n m} x_{n m}, \\
& F_{4}\left(x_{11}, x_{12}, \ldots, x_{i j}, \ldots, x_{n m}\right)=D_{11} x_{11}+D_{12} x_{12}+\cdots+D_{n m} x_{n m},
\end{aligned}
$$

where $B_{11}, B_{12}, \ldots, B_{n m} ; C_{11}, C_{12}, \ldots, C_{n m}$; and $D_{11}, D_{12}, \ldots, D_{n m}$ are the coefficients of emissions reduction. $B_{i j}, C_{i j}$, and $D_{i j}=$ equivalent avoided emissions of $\mathrm{CO}_{2}, \mathrm{NO}_{\mathrm{X}}$, and $\mathrm{SO}_{2}$, respectively, for energy unit, when using the RE source $i$ for all $B_{i j} \geq 0, C_{i j} \geq 0$, and $D_{i j} \geq 0$, so that the maximization of the functions optimally reduces the avoided emissions.

Coefficients were obtained from official national organizations. Unlike the ones obtained from $F_{1}$, these coefficients were quantitative values.

\section{$F_{5}:$ Cost minimization}

The linear function of costs was as follows:

$$
F_{5}\left(x_{11}, x_{12}, \ldots, x_{i j}, \ldots, x_{n m}\right)=E_{11} x_{11}+E_{12} x_{12}+\cdots+E_{n m} x_{n m},
$$

where $E_{11}, E_{12}, \ldots, E_{n m}$ are the cost coefficients.

TABLE III. Electrification coefficients in Peru by region [data from United Nations Development Programme (UNDP)]. ${ }^{4,18}$

\begin{tabular}{lccc}
\hline \hline Region & Region coefficient & Province with the lowest electrification & Province coefficient \\
\hline Cajamarca & 0.4 & San Pablo & 0.19 \\
Huánuco & 0.4 & Puerto Inca & 0.11 \\
Amazonas & 0.5 & Condorcanqui & 0.10 \\
Ayacucho & 0.5 & Vilcas Huamán & 0.19 \\
Huancavelica & 0.6 & Castrovirreyna & 0.37 \\
Apurímac & 0.6 & Cotabambas & 0.31 \\
Puno & 0.6 & Lampa & 0.38 \\
San Martín & 0.6 & El Dorado & 0.36 \\
Loreto & 0.6 & Datem del Marañón & 0.27 \\
Cusco & Chumbivilcas & 0.19 \\
Ucayali & 0.6 & Atalaya & 0.32 \\
Piura & 0.7 & Ayabaca & 0.27 \\
Madre de Dios & 0.7 & Manu & 0.45 \\
Pasco & 0.7 & Oxapampa & 0.51 \\
La Libertad & 0.7 & Julcán & 0.20 \\
Ancash & 0.7 & C, F, Fitzcarrald & 0.31 \\
Junín & 0.7 & Satipo & 0.39 \\
Lambayeque & 0.7 & Lambayeque & 0.50 \\
Ica & 0.8 & Pisco & 0.65 \\
Moquegua & 0.8 & General Sánchez Cerro & 0.56 \\
Tumbes & 0.8 & Zarumilla & 0.76 \\
Tacna & 0.8 & Tarata & 0.63 \\
Arequipa & La Unión & 0.41 \\
Lima & 0.8 & Canta & 0.59 \\
\hline \hline
\end{tabular}




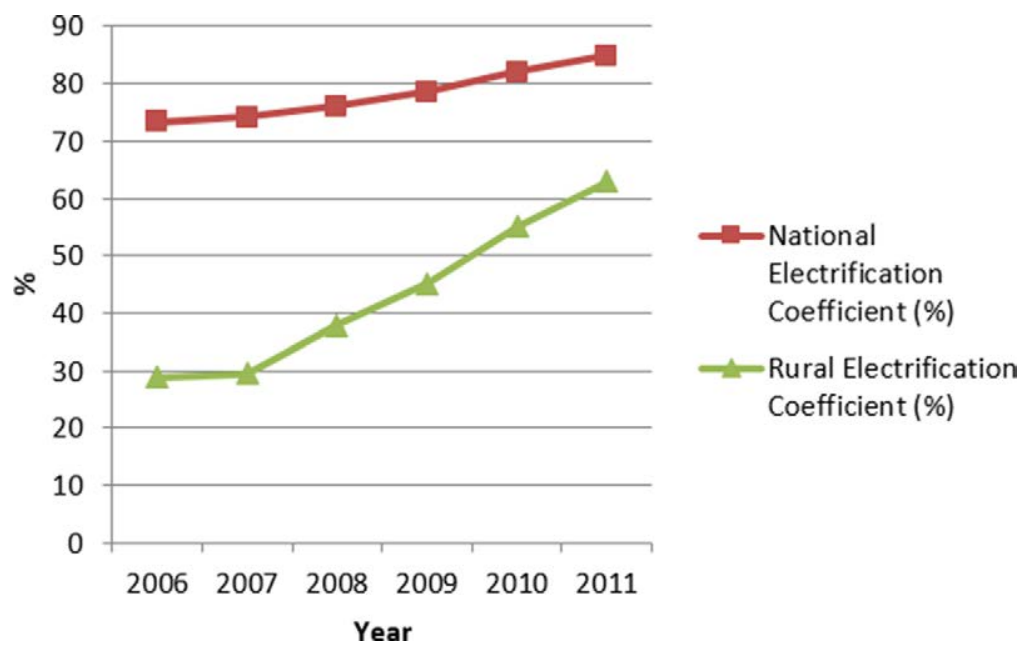

FIG. 2. National electrification coefficients in Peru to 2011. Data from MINEM. ${ }^{16}$

$E_{i j}=\mathrm{LCOE}$ (levelized cost of electricity) (USD/ktoe) from each energy alternative for all $E_{i j} \geq 0$, so that the minimization of the function $F_{5}$ reduces the costs. In the same way as the coefficients $B_{i j}, C_{i j}$ and $D_{i j}$, the coefficients $E_{i j}$ were obtained from official sources.

\section{$F_{6}:$ Maximization of renewable energy technologies for sustainable supply of energy in IRC}

This innovative function considers electric energy supply in IRCs, which is the main electrification problem in Cajamarca province. It has been developed gathering the experience of AMP. This social micro-utility company is the major supplier of distributed photovoltaic solar power in Peru. Currently its intervention is concentrated in the Cajamarca region.

According to the last Population and Housing Census of Peru conducted in 2007, 25.9\% of households did not have electric energy. This percentage reaches its greatest value in the Cajamarca region (Table III), where $59.8 \%$ of households did not have that service. ${ }^{4}$ The high degree of dispersal of households in the rural area makes grid extension economically inviable and fuelled the search for new energy technologies. ${ }^{17}$

The 2013-2022 National Rural Electrification Plan produced by the Ministry of Energy and Mining (MINEM) ${ }^{19}$ in concordance with the Universal Energy Access Plan, established a policy for the sector with the aim of raising the rural electrification rate from $87 \%$ to $95 \%$ by 2016 (Fig. 2). Peru is making an effort to provide this energy access with tenders addressed to install solar home systems; mini-grids with hydro, solar, and wind; and grid extension. ${ }^{20}$

Experience had demonstrated that technological solutions are not enough to ensure energy access in IRCs. It is also fundamental to design a management model that considers social and cultural aspects from IRCs in order to achieve sustainability in this kind of project.

Figure 3 shows a tree of means and ends elaborated by ACCIONA Microenergía Perú. It incorporates criteria mentioned above and it could provide the keys for choosing the most appropriate alternative: that is to say, the alternative that supplies energy in an integral, reliable, and efficient way.

According to the above, the equation for $F_{6}$ was obtained:

$$
F_{6}\left(x_{11}, x_{12}, \ldots, x_{i j}, \ldots, x_{n m}\right)=G_{11} x_{11}+G_{12} x_{12}+\cdots+G_{n m} x_{n m} .
$$

Coefficients were obtained following the same method as for $F_{1}$. The criteria or comparison levels were determined as per the experience of local suppliers and consulting experts (Table IV). 
TABLE IV. Precedence constraints of objective function $F_{6}$, brief description, and numerical evaluation.

\begin{tabular}{|c|c|c|}
\hline Level and criteria & Description & Evaluation \\
\hline \multicolumn{3}{|l|}{ Technologic } \\
\hline Long lifetime & $\begin{array}{l}\text { Refers to the durability of the equipment. This criterion influences eco- } \\
\text { nomic aspects of the projects and, therefore, their sustainability. }\end{array}$ & The scale used ranges from 5 (long lifetime) to 1 (short lifetime). \\
\hline Battery required & $\begin{array}{l}\text { Most technologies use batteries for energy storage. However, in this } \\
\text { study, it has been considered as favorable to dispense with them for } \\
\text { cost and environmental issues. }\end{array}$ & $\begin{array}{c}\text { The scale used ranges from } 5 \text { (battery is not required) to } 1 \text { (battery is } \\
\text { required). }\end{array}$ \\
\hline AC output & $\begin{array}{l}\text { AC output is considered favorable in the technologies to evaluate. AC } \\
\text { output allows the use of a higher quantity of devices by the consumer. } \\
\text { On the other hand, the cost of DC devices is still high. }\end{array}$ & $\begin{array}{c}\text { The scale used ranges from } 1 \text { (low possibility) to } 5 \text { (high possibility to } \\
\text { use this characteristic). }\end{array}$ \\
\hline Modularity of system & $\begin{array}{l}\text { Technologies with this characteristic allow the user to expand their } \\
\text { power generation capacity through serial or parallel connections. }\end{array}$ & $\begin{array}{l}\text { The scale used ranges from } 5 \text { (high possibility of modularity) to } 1 \text { (low } \\
\text { possibility of modularity). }\end{array}$ \\
\hline Easy hybridization & $\begin{array}{l}\text { This characteristic ensures the continuity of energy production of the } \\
\text { systems during day and night. Solar and wind energy are often hybrid- } \\
\text { ized in home systems. }\end{array}$ & $\begin{array}{l}\text { The scale used ranges from } 1 \text { (systems with difficult hybridization) to } \\
\qquad 5 \text { (systems with easy hybridization). }\end{array}$ \\
\hline Required import equipment & $\begin{array}{l}\text { The systems have parts that can be obtained locally, but other ones } \\
\text { have to be imported. According to this, there will be advantages of one } \\
\text { technology over another. }\end{array}$ & $\begin{array}{l}\text { The scale used ranges from } 1 \text { (systems that require import all their } \\
\text { parts) to } 5 \text { (systems that require import no parts or few). }\end{array}$ \\
\hline \multicolumn{3}{|l|}{ Economic } \\
\hline Lower LCOE & $\begin{array}{l}\text { Levelized cost of energy (LCOE) is the cost of the alternative to gener- } \\
\text { ate } 1 \text { tonne of oil equivalent during its lifetime. }\end{array}$ & $\begin{array}{c}\text { The scale used ranges from } 1 \text { (systems that require import all their } \\
\text { parts) to } 5 \text { (systems that not require import of parts or only of few } \\
\text { parts). }\end{array}$ \\
\hline Lower maintenance cost & $\begin{array}{l}\text { In isolated rural electrification, an important component of mainte- } \\
\text { nance costs is the need to move to households. This criterion was } \\
\text { evaluated. }\end{array}$ & $\begin{array}{l}\text { The scale used ranges from } 5 \text { (lower maintenance cost) to } 1 \text { (higher } \\
\text { maintenance cost). }\end{array}$ \\
\hline Scalability and replicability & $\begin{array}{l}\text { Scalability involves the growth of the project in order to increase its } \\
\text { social impact in a specific area, while replicability is related to the } \\
\text { implementation of the project in a different geographic and social } \\
\text { context. }\end{array}$ & $\begin{array}{l}\text { The scale used ranges from } 1 \text { (low scalability and replicability) to } 5 \\
\text { (high scalability and replicability). }\end{array}$ \\
\hline \multicolumn{3}{|l|}{ Operation } \\
\hline Easy installation & $\begin{array}{l}\text { An easy installation increases the efficiency of the time during the exe- } \\
\text { cution of the projects, which has an impact on costs. On the other } \\
\text { hand, it avoids specialized training. }\end{array}$ & $\begin{array}{l}\text { The scale used ranges from } 1 \text { (systems with difficult installation) to } 5 \\
\text { (systems with easy installation). }\end{array}$ \\
\hline
\end{tabular}


TABLE IV. (Continued.)

\begin{tabular}{|c|c|c|}
\hline Level and criteria & Description & Evaluation \\
\hline Training required & $\begin{array}{l}\text { Refers to the degree of training that users will require to operate the } \\
\text { systems. New technologies offer systems that can be installed by the } \\
\text { users themselves. }\end{array}$ & $\begin{array}{l}\text { The scale used ranges from } 5 \text { (only elemental training is required) to } 1 \\
\text { (specialized training is required). }\end{array}$ \\
\hline Security against theft & $\begin{array}{l}\text { Refers to the security of the systems installed in the household. The } \\
\text { outdoor location of some of its components could increase the likeli- } \\
\text { hood of theft. }\end{array}$ & $\begin{array}{l}\text { The scale used ranges from } 1 \text { (low security against theft) to } 5 \text { (high } \\
\text { security against theft). }\end{array}$ \\
\hline \multicolumn{3}{|l|}{ Planning } \\
\hline Acceptance and social participation & $\begin{array}{l}\text { The acceptance and participation of beneficiaries in the management } \\
\text { model of rural electrification projects is a key factor in the sustainabil- } \\
\text { ity of this type of project. }\end{array}$ & $\begin{array}{c}\text { The scale used ranges from } 1 \text { (low acceptance and social participation) } \\
\text { to } 5 \text { (high acceptance and social participation). }\end{array}$ \\
\hline Direct social impact & $\begin{array}{l}\text { Impact of the new technology on the end user. For example, having } \\
\text { electricity for the first time or having access to media, such as televi- } \\
\text { sion or radios. }\end{array}$ & $\begin{array}{l}\text { The scale used ranges from } 1 \text { (low direct social impact) to } 5 \text { (high } \\
\text { direct social impact). }\end{array}$ \\
\hline Generation of permanent employment & $\begin{array}{l}\text { This characteristic is related to the employment that the project can } \\
\text { generate in the area, for example, providing maintenance to the } \\
\text { installed systems or providing equipment through small and medium- } \\
\text { sized enterprises. }\end{array}$ & $\begin{array}{l}\text { The scale used ranges from } 1 \text { (low generation of permanent employ- } \\
\text { ment) to } 5 \text { (high generation of permanent employment). }\end{array}$ \\
\hline Existing tariff legislation & $\begin{array}{l}\text { Refers to the existing regulation in the country where the intervention } \\
\text { takes place, related to the renewable energy source used. }\end{array}$ & $\begin{array}{l}\text { The scale used ranges from } 1 \text { (absence or weak tariff legislation) to } 5 \\
\text { (existence of solid tariff legislation). }\end{array}$ \\
\hline \multicolumn{3}{|l|}{ Environmental } \\
\hline Low environmental impact & $\begin{array}{l}\text { Refers to a valuation applied to the materials with which the techno- } \\
\text { logical equipment is made. The type of battery used is of special } \\
\text { relevance. }\end{array}$ & $\begin{array}{l}\text { The scale used ranges from } 5 \text { (low environmental impact) to } 1 \text { (high } \\
\text { environmental impact). }\end{array}$ \\
\hline Lower waste generation & $\begin{array}{l}\text { This characteristic refers to the generation of waste during the installa- } \\
\text { tion of the equipment. }\end{array}$ & $\begin{array}{c}\text { The scale used ranges from } 5 \text { (low environmental impact) to } 1 \text { (high } \\
\text { environmental impact). }\end{array}$ \\
\hline Availability of energy source & Potential for the use of renewable energy sources in the study area. & $\begin{array}{c}\text { The scale used ranges from } 5 \text { (high availability of the energy resource) } \\
\text { to } 1 \text { (low availability of the energy resource). }\end{array}$ \\
\hline
\end{tabular}




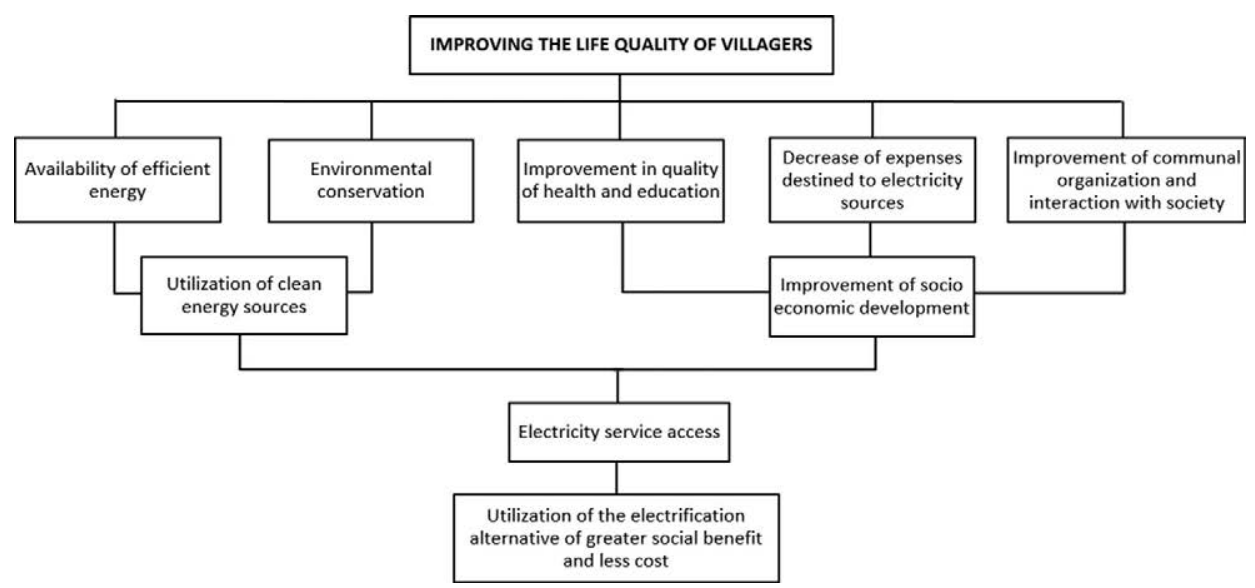

FIG. 3. Tree of means and ends in rural electrification projects. Reproduced with permission from ACCIONA Micronergía Perú (AMP). Perfil del proyecto de electrificación de las localidades de la zona rural de Cachachi, La Encañada, Namora, San Pablo, Tumbaden e Ichocan, en la región Cajamarca, empleando energía solar fotovoltaica (Cajamarca, 2011).

\section{Restrictions}

The restrictions were grouped in four types according to origin.

\section{Restrictions due to non-electric energy use $\left(\mathrm{S}_{\mathrm{i}}\right)$}

$S_{j}$ : non-electric energy demand, calculated as: $S_{j}=p_{j}-i_{j}$; where $p_{j}$ is the energy demand corresponding to sector $j$ and $i_{j}$ is the amount of fossil fuel that renewable sources cannot replace.

\section{Restrictions due to electric energy use $\left(\mathrm{S}_{\mathrm{e}}\right)$}

$S_{e}$ : electric energy demand, calculated as: $S_{e}=p_{e}-i_{e}$; where $p_{e}$ is the total electric energy demand and $i_{e}$ is the amount of fossil fuel that renewable sources cannot replace.

\section{Restrictions due to the RE potential production $\left(\mathrm{P}_{\mathrm{i}}\right)$}

$P_{i}$ : potential application of renewable source $i$ in the corresponding sector.

\section{Restrictions due to the current RE systems $\left(\mathrm{R}_{\mathrm{i}}\right)$}

$R_{i}$ : minimum amount of conventional energy that renewable sources can replace, as RE has already replaced this amount.

Table V shows the mathematical form of the constraints.

\section{Compromise programming}

$\mathrm{Yu}^{22}$ and Zeleny ${ }^{23}$ used the concept of the ideal point as a reference to the decision maker. According to those authors, this point is one in which each objective reaches its optimum value. The ideal point can be represented by the following vector:

TABLE V. Restrictions (prepared by the authors based on García ${ }^{15}$ ).

\begin{tabular}{llcc}
\hline \hline Non-electric energy use & Electric energy use & RE potential production & Current RE systems \\
\hline$\sum_{j=1}^{n} X_{i j} \leq S_{j} \quad$ (9) $\quad \sum_{i=1}^{l} X_{i e} \leq S_{e}$ (10) $\quad X_{i e}+\sum_{j=1}^{m} X_{i j} \leq P_{i} \quad(11)$ & $X_{i e}+\sum_{j=1}^{m} X_{i j} \leq R_{i} \quad(12)$ \\
\hline \hline
\end{tabular}




$$
F^{*}=\left(F_{1}^{*}, \ldots F_{i}^{*}, \ldots F_{n}^{*}\right),
$$

where $F_{i}^{*}$ is the optimum value of the objective function $F_{i}(x)$ and $x$ complies with the restrictions of the problem.

Taking into account that the ideal point is nonachievable, the decision maker will search for an efficient point as close as possible to it. Romero ${ }^{24}$ introduced the concept of distance in order to find out the set of solutions closest to the ideal point. The degree of closeness between the objective $F_{j}(x)$ and its ideal is given by the distance $d_{j}$ :

$$
d_{j}=\left[F_{j}^{*}-F_{j}(x)\right] .
$$

Since the objectives usually have different units, and in order to avoid biases towards objectives with large numbers, this distance should be normalized:

$$
d_{j}=\frac{\left[F_{j}^{*}-F_{j}(x)\right]}{\left[F_{j}^{*}-F_{* j}\right]},
$$

where $F_{* j}$ is the anti-ideal point for the $j$ th objective. By making a generalization of the Euclidean distance, defined as the shorter distance between two points, a family of metrics is obtained:

$$
L_{p}=\left[\sum_{j=1}^{n}\left[\frac{F_{j}^{*}-F_{j}(x)}{F_{j}^{*}-F_{* j}}\right]^{p}\right]^{1 / p} .
$$

The parameter $p$ represents the metric that defines the family of distance functions. If we consider $W_{j}$ as the weights representing the relative importance of the $j$ th objective to the decision maker, the compromise programming becomes the following optimization problem:

$$
\operatorname{Min} L_{p}=\left[\sum_{j=1}^{n} W_{j}^{p}\left[\frac{F_{j}^{*}-F_{j}(x)}{F_{j}^{*}-F_{* j}}\right]^{p}\right]^{1 / p} .
$$

Zeleny ${ }^{23}$ calls the set bounded by points $L_{1}$ and $L_{\infty}$ the compromise set. According to Romero, ${ }^{24} L_{1}$ corresponds to the point of maximum efficiency, and $L_{\infty}$ is a well-balanced solution. This paper calculates $L_{1}$ and $L_{\infty}$, called commonly Manhattan and Chebysev distances, respectively. Likewise, intermediate distances have been obtained $\left(L_{k}\right)$ from the compromise set. Considering that the SEPLAN model has six objective functions, the mentioned distances are shown in Table VI.

TABLE VI. Normalized distances to be minimized.

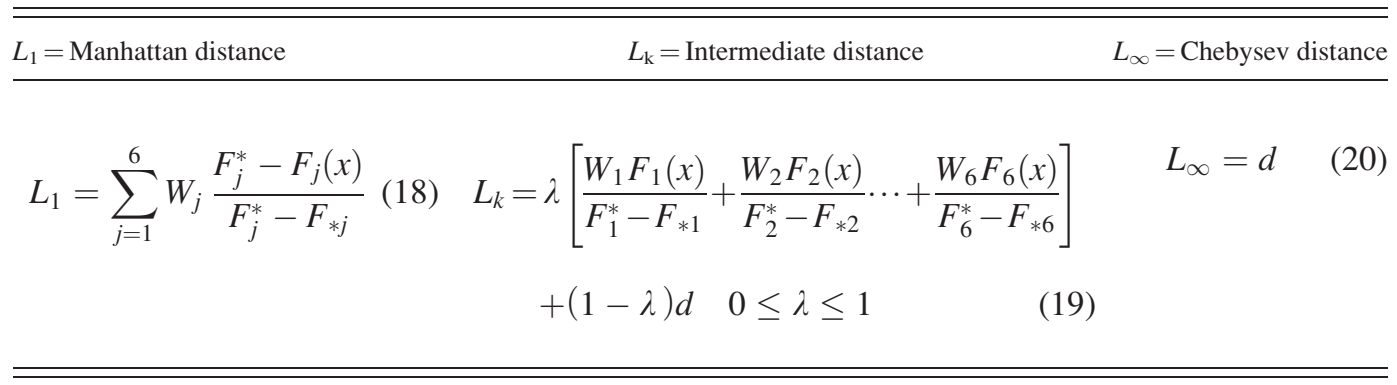


Note that $L_{1}$ is minimized according to above restrictions. In order to minimize $L_{\infty}$, the same restrictions have been taken into account, plus those below:

$$
\begin{aligned}
& W_{1} \frac{F_{1}^{*}-F_{1}(x)}{F_{1}^{*}-F_{* 1}} \leq d, \\
& W_{2} \frac{F_{2}^{*}-F_{2}(x)}{F_{2}^{*}-F_{* 2}} \leq d, \\
& W_{3} \frac{F_{3}^{*}-F_{3}(x)}{F_{3}^{*}-F_{* 3}} \leq d, \\
& W_{4} \frac{F_{4}^{*}-F_{4}(x)}{F_{4}^{*}-F_{* 4}} \leq d, \\
& W_{5} \frac{F_{5}^{*}-F_{5}(x)}{F_{5}^{*}-F_{* 5}} \leq d, \\
& W_{6} \frac{F_{6}^{*}-F_{6}(x)}{F_{6}^{*}-F_{* 6}} \leq d .
\end{aligned}
$$

$L_{k}$ is minimized according the same seven restrictions indicated to minimize $L_{\infty}$. These distances are obtained by varying the values of $\lambda$ from 0 to 1 . For $\lambda=1$, we will obtain the value $L_{1}$, and for $\lambda=0$, we will obtain the value $L_{\infty}$ (see Table VI).

\section{STUDY CASE: CAJAMARCA PROVINCE}

\section{A. Demand projection}

Table VII shows the forecasted consumption for 2025 of the main energy sources in Cajamarca province. These consumptions were obtained according to Energy Balance 2014, published by official sources. ${ }^{25}$ The sectors of greatest impact on energy use have been taken into account. The forecasted consumptions were according to the National Energy Plan 2014-2025 of MINEM. This Plan considers two scenarios of GDP growth: $4.5 \%$ and $6.5 \%{ }^{14}$ Taking into account the historical consumption of electricity, the forecasts were calculated according to the average results of the summative and multiplicative models. ${ }^{26}$ Then, a regression equation was generated obtaining for the increase of $6.5 \%$ of GDP the most favourable $R^{2}$. Finally, the forecasted consumptions for the different energy sources were calculated. The estimated error in the demand forecasting in 2025 was $\sim 12 \%$.

The Peruvian regulatory framework considers that every 5 years, power generation with

\begin{tabular}{|c|c|c|c|c|c|c|c|}
\hline Sector & Electricity & Diesel B5 & Gasohol (7.8\%) & DB5 S-50 & Firewood & Charcoal & Geothermic \\
\hline Transport & & 14.58 & 6.11 & 0.004 & & & \\
\hline Commercial & 2.94 & & & & & & \\
\hline Industrial & 13.59 & 25.86 & 0.09 & & & & \\
\hline Domestic & 9.83 & & & & 12.69 & 2.70 & 0.205 \\
\hline Public lighting & 1.23 & & & & & & \\
\hline
\end{tabular}
renewable energy will be 5\% of the national electricity consumption. ${ }^{27}$ According to that and taking into account the potential of renewable resources in the area, the authors have established a substitution percentage of $15 \%$ in this sector. It should be noted that the $5 \%$ mentioned does not include large hydro, which is considered as a conventional renewable source.

TABLE VII. Energy consumption forecast by sectors in the province of Cajamarca, Peru, year 2025 (ktoe). ${ }^{25}$ 
TABLE VIII. Substitutable energy in 2025 (ktoe).

\begin{tabular}{lccc}
\hline \hline Sector & Electricity & Diesel B5 & Gasohol (7.8\%) \\
\hline Transport & & 0.73 & 0.31 \\
Commercial & 0.44 & & \\
Industrial & 2.04 & 2.59 & \\
Domestic & 1.47 & & \\
Public lighting & 0.19 & & \\
\hline \hline
\end{tabular}

With regard to transport fuels in Peru, since 2011, diesel B5 and gasohol (7.8\%) have been traded. ${ }^{28}$ Hence, a substitution of $5 \%$ is estimated of the transport fuel consumption used in Cajamarca province for liquid biofuels (100\% concentration). It is important to note that $\sim 80 \%$ of diesel B5 consumption is used for generating electricity power for mining, which is the most important economic activity in Cajamarca province.

According to this, the fossil energy (ktoe) that can be substitutable for RE was estimated (Table VIII).

This calculation has taken into account the same processes to power generation in order to meet demands of fossil energy that will not be replaced by renewable energy in the year 2025 . For instance, industrial energy demand will continue to be supplied by thermal power plants; hydroelectric centrals will meet the commercial, domestic, and public lighting electric demand; and the transport sector will continue to use the same fuels.

\section{B. Renewable energy potential}

The RE with the greatest potential to be utilized is photovoltaic solar energy. The Cajamarca region is located in an area with high availability of daily solar energy, from 5.5 to $6.0 \mathrm{kWh} / \mathrm{m}^{2}{ }^{29}$ In 2013, the Peruvian government implemented a tender for the installation of 500000 solar systems in rural areas, and the winning company in 2015 began the project implementation. ${ }^{30}$ There are $\sim 13000$ families without access to electricity in Cajamarca province. ${ }^{31}$ Currently, the largest supplier of domiciliary photovoltaic solar energy in the region is ACCIONA Microenergía Perú, serving 3900 families. ${ }^{32}$

With regard to wind power, there are areas in the region with a wind speed of $7-8 \mathrm{~m} / \mathrm{s}^{33}$ There are projects at a household level developed by Practical Solutions-ITDG (Intermediate Technology Development Group). They are mainly hybrid systems but still on a small scale.

Another alternative with great potential is mini-hydro. According to an estimate made by the authors based on local information, 6.2 MW could be utilized in the province. Practical Solutions-ITDG has installed 40 micro-hydropower plants in the region. ${ }^{34}$

Regarding the use of biomass, the program access to energy EnDev GIZ has installed 18545 improved cooking stoves in the province of Cajamarca. It has been done with the support of public and private institutions. ${ }^{35}$ This is the reason it has been estimated that the consumption of firewood for cooking food will remain stable in the coming years.

There is an important potential for the use of castor (Ricinus communis L.) in order to produce biodiesel in the Condebamba Valley. A plantation of 2000 hectares and a production of 13061 of oil per hectare has been estimated. ${ }^{36}$

Another opportunity to introduce renewable energies is the substitution of diesel B5 that in Yanacocha mining is using for power generation. ${ }^{37}$ A substitution of $10 \%$ of its consumption by photovoltaic solar energy has been planned.

\section{Establishment of restrictions}

The restrictions for this case of study are shown in Tables IX and X. Note that the electrical sector is analysed separately due to its special characteristics. 
TABLE IX. Restrictions based on energy demand and RE real potential (in ktoe).

\begin{tabular}{ll}
\hline \hline ST & $\leq 1.66^{\mathrm{a}}$ \\
FW & $\leq 12.70^{\mathrm{b}}$ \\
CH & $\leq 2.70^{\mathrm{b}}$ \\
LB & $\leq 1.03^{\mathrm{c}}$ \\
GEO & $\leq 0.20^{\mathrm{d}}$ \\
Electricity & \\
PV + WE + SH & $\leq 6.72$ \\
PV & $\leq 3.36^{\mathrm{e}}$ \\
WE & $\leq 2.39^{\mathrm{f}}$ \\
SH & $\leq 3.72 \mathrm{~g}$ \\
\hline
\end{tabular}

${ }^{\mathrm{a}}$ Calculated based on $\mathrm{INEI}^{38}$ and Orbegozo and Arivilca. ${ }^{40}$

${ }^{\mathrm{b}}$ Calculated based on INEI, ${ }^{3}$ Díaz et al. ${ }^{35}$ and FAO. ${ }^{44}$

${ }^{\mathrm{c}}$ Calculated based on OSINERGMIN, ${ }^{25}$ Campos and Salazar, ${ }^{36}$ and Pérez et al ${ }^{48}$

${ }^{\mathrm{d}}$ Calculated based on INEI ${ }^{38}$ and JICA. ${ }^{41}$

${ }^{\mathrm{e}}$ Calculated based on SENAMHI, ${ }^{29}$ AMP, ${ }^{21}$ OSINERGMIN,,${ }^{31}$ INEI,${ }^{38}$ and Hidrandina. ${ }^{39}$

${ }^{\mathrm{f}}$ Calculated based on MINEM, ${ }^{33}$ Soluciones Prácticas-ITDG, ${ }^{42}$ and Departamento de Energía, EE.UU. ${ }^{43}$

${ }^{\mathrm{g}}$ Calculated based on IRENA, ${ }^{30}$ López, ${ }^{45}$ Gobierno Regional de Cajamarca,${ }^{46}$ and Soluciones Prácticas-ITDG. ${ }^{47}$

TABLE X. Restrictions based on the already existing RE (in ktoe).

\begin{tabular}{lll}
\hline \hline ST & $\geq$ & $0.10^{\mathrm{a}}$ \\
FW & $\geq$ & $1.70^{\mathrm{a}}$ \\
CH & $\geq$ & $1.00^{\mathrm{a}}$ \\
GEO & $\geq$ & $0.05^{\mathrm{a}}$ \\
LB & $\geq$ & $0.74^{\mathrm{b}}$ \\
PV & $\geq$ & $2.64^{\mathrm{c}}$ \\
WE & $\geq$ & $0.01^{\mathrm{d}}$ \\
SH & $\geq$ & $0.5^{\mathrm{d}}$ \\
\hline
\end{tabular}

${ }^{\mathrm{a}}$ Estimated by the authors.

${ }^{\mathrm{b}}$ Minimum amount for substitution of diesel B5 in transport. Currently no production of LB exists.

${ }^{\mathrm{c}}$ Minimum amount for substitution of diesel B5 used for generate electric energy by Yanacocha mine. An installed power of 0.06 ktoe was considered for PV in Cajamarca province.

${ }^{\mathrm{d}}$ Estimated based on Soluciones Prácticas-ITDG. ${ }^{34}$

\section{The optimization equations}

\section{$\mathrm{F}_{1}$ : Maximization of RE potential capacity}

In order to determine the coefficients corresponding to $F_{1}$ function, the authors have established precedence constraints (Table XI).

$N$ and $M$ are then calculated according to (2) and (3) and (Table XII).

Once the coefficients are applied, this is the final equation for $F_{1}$ :

$$
F_{1}=2.5 \mathrm{PV}+2.5 \mathrm{SH}+2 \mathrm{ST}+2 \mathrm{WE}+2 \mathrm{GEO}+1.5 \mathrm{CH}+1.5 \mathrm{FW}+\mathrm{LB} .
$$

\section{$\mathrm{F}_{2}, \mathrm{~F}_{3}$, and $\mathrm{F}_{4}$ : Environmental impact minimization}

In Peru, the largest greenhouse gases (GHG) emissions are produced by deforestation and land-use change, which release $\mathrm{CO}_{2}$ into the atmosphere. Although GHG emissions from Peru represent $0.4 \%$ of global emissions, they continue to grow. They are related to demographic trends, consumption patterns, and economic and technological changes, among other factors. ${ }^{49}$

Electricity generation from renewable energy resources (RER) in the country have mitigated the emission of 2084 thousands of tonnes of $\mathrm{CO}_{2}$ equivalent $(\mathrm{tCO} 2 \mathrm{e})$. This occurred from the start of the operations of the first RER central until $2013 .^{50}$ 
TABLE XI. Precedence constraints of $F_{1}$.

\begin{tabular}{lcccccccc}
\hline \hline Constraint & PV & ST & WE & FW & CH & LB & SH & GEO \\
Technological demand & 4 & 4 & 4 & 2 & 2 & 2 & 5 & 3 \\
Potential investigation & 3 & 3 & 3 & 4 & 4 & 3 & 4 & 2 \\
Know-how up-to-date & 5 & 4 & 3 & 3 & 5 & 2 & 4 & 4 \\
Technology improvement (possibly locally manufactured equipment) & 4 & 3 & 3 & 3 & 3 & 3 & 3 & 3 \\
Current technological level to apply the alternative energy & 4 & 2 & 3 & 2 & 3 & 2 & 4 & 2 \\
Operating time & 5 & 5 & 4 & 5 & 5 & 2 & 4 & 5 \\
Implementation issues & 4 & 5 & 4 & 5 & 4 & 4 & 3 & 5 \\
Integration possibility & 5 & 4 & 5 & 5 & 5 & 5 & 5 & 5 \\
Potential demand & 4 & 5 & 4 & 3 & 3 & 3 & 5 & 4 \\
Supply availability & 5 & 5 & 3 & 5 & 4 & 2 & 3 & 4 \\
Political interest & 5 & 4 & 5 & 2 & 2 & 5 & 5 & 4 \\
Current price compared with conventional energies & 2 & 3 & 3 & 2 & 2 & 2 & 5 & 4 \\
Social demand & 4 & 4 & 4 & 2 & 2 & 2 & 5 & 4 \\
Legislative forecast & 5 & 3 & 4 & 3 & 3 & 5 & 4 & 3 \\
Environmental impact & 4 & 5 & 5 & 2 & 2 & 5 & 2 & 5 \\
\hline \hline
\end{tabular}

TABLE XII. Calculation of $N$ and $M$ factors.

\begin{tabular}{lccc}
\hline \hline Alternative & $M$ & $N$ & Coefficient \\
\hline PV & $1.5 \times 10^{9}$ & $1.5 \times 10^{3}$ & 2.5 \\
SH & $8.6 \times 10^{8}$ & $3.5 \times 10^{3}$ & 2.5 \\
ST & $5.2 \times 10^{8}$ & $1.0 \times 10^{4}$ & 2 \\
WE & $3.7 \times 10^{8}$ & $4.7 \times 10^{4}$ & 2 \\
GEO & $2.8 \times 10^{8}$ & $2.8 \times 10^{4}$ & 2 \\
CH & $2.1 \times 10^{7}$ & $6.6 \times 10^{5}$ & 1.5 \\
FW & $1.3 \times 10^{7}$ & $6.6 \times 10^{5}$ & 1.5 \\
LB & $8.6 \times 10^{6}$ & $8.8 \times 10^{5}$ & 1 \\
\hline \hline
\end{tabular}

In Cajamarca, the main GHG emissions are produced by fuel combustion for transport and thermal power plants. ${ }^{46}$ Besides the $\mathrm{CO}_{2}$, these activities produce two air pollutants: $\mathrm{NO}_{\mathrm{X}}$ and $\mathrm{SO}_{2}$. The $\mathrm{NO}_{\mathrm{X}}$ emissions are mainly due to the poor state of vehicles in the city. ${ }^{51}$

In accordance with the above and taking into account the GHG National Inventory of Peru in $2000,{ }^{52}$ the coefficients for the functions of $\mathrm{CO}_{2}, \mathrm{NO}_{\mathrm{X}}$, and $\mathrm{SO}_{2}$ were $7.004 \mathrm{tCO}_{2} /$ toe, 0.020 $\mathrm{tNO}_{\mathrm{X}} /$ toe, and $0.029 \mathrm{tSO}_{2} /$ toe, respectively.

Table XIII shows a summary of the life cycle emissions corresponding to the different types of renewable energy that have been considered to calculate the coefficients.

Regarding to $\mathrm{LB}$, the amount of avoided $\mathrm{CO}_{2}$ emissions has been calculated considering that diesel B5 is mainly the fuel to substitute. According to this and keeping in mind that the life cycle of $\mathrm{CO}_{2}$ emissions for $\mathrm{LB}$ is $0.8129 \mathrm{tCO}_{2} /$ toe, ${ }^{54}$ avoided $\mathrm{CO}_{2}$ emissions were estimated as $1.8687 \mathrm{tCO}_{2} /$ toe. When biofuel replaces gasoline and diesel in the transport sector,

TABLE XIII. RE life cycle $\mathrm{CO}_{2}$ emissions: $\mathrm{tCO}_{2} /$ toe, $\mathrm{tNO}_{\mathrm{x}} /$ toe, $\mathrm{tSO}_{2} /$ toe. ${ }^{53}$

\begin{tabular}{lcccccr}
\hline \hline Life cycle emission & PV & ST & WE & FW & SH & GEO \\
\hline $\mathrm{CO}_{2}$ & 1.1512 & 0.1558 & 0.1186 & 0.1628 & 0.1512 & 0.4395 \\
$\mathrm{NO}_{\mathbf{X}}$ & 0.004 & 0.0085 & 0.0004 & 0.003 & 0.0006 & 0.0022 \\
$\mathrm{SO}_{2}$ & 0.0033 & 0.0005 & 0.0005 & 0.0003 & 0.0003 & 0.0007 \\
\hline \hline
\end{tabular}


$\mathrm{SO}_{2}$ emissions are reduced. However, in the case of NOx, emissions reduction depends on the substitution pattern and technology. ${ }^{13}$ Many studies report higher $\mathrm{NO}_{\mathrm{x}}$ emissions with biodiesel than petroleum diesel. ${ }^{55}$ For this reason, $\mathrm{NO}_{\mathrm{x}}$ emissions have not been considered. Avoided $\mathrm{SO}_{2}$ emissions were obtained from the GHG National Inventory of Peru in the year $2000 .{ }^{52}$ This value was $0.0071 \mathrm{tSO}_{2} /$ toe.

Note that national environmental indicators were used due to the scarce information in the Cajamarca province. This was a limitation for the calculation of these coefficients.

Next, the corresponding functions are shown:

$$
\begin{aligned}
F_{2}= & 6.544 \mathrm{PV}+7.004 \mathrm{ST}+6.884 \mathrm{SH}+2.7 \mathrm{LB}+6.374 \mathrm{CH}+7.004 \mathrm{GEO}+6.884 \mathrm{WE} \\
& +6.484 \mathrm{FW}, \\
F_{3}= & 0.0181 \mathrm{PV}+0.0198 \mathrm{ST}+0.0197 \mathrm{SH}+0.0115 \mathrm{CH}+0.0198 \mathrm{GEO}+0.0194 \mathrm{WE} \\
& +0.0115 \mathrm{FW}, \\
F_{4}= & 0.026 \mathrm{PV}+0.029 \mathrm{ST}+0.029 \mathrm{SH}+0.0058 \mathrm{LB}+0.0286 \mathrm{CH}+0.029 \mathrm{GEO}+0.0285 \mathrm{WE} \\
+ & 0.0286 \mathrm{FW} .
\end{aligned}
$$

\section{$\mathrm{F}_{5}$ : Cost minimization of substitution of RE for existing conventional energy}

LCOE value (USD/toe) was obtained from official sources for each energy alternative. ${ }^{56}$ According to this, the equation obtained was the following:

$$
F_{5}=0.11 \mathrm{ST}+0.11 \mathrm{PV}+0.09 \mathrm{WE}+0.06 \mathrm{FW}+0.06 \mathrm{CH}+0.093 \mathrm{LB}+0.04 \mathrm{SH}+0.04 \mathrm{GEO} .
$$

\begin{tabular}{|c|c|c|c|c|c|c|c|c|}
\hline Factor & PV & $\mathrm{ST}$ & WE & FW & $\mathrm{CH}$ & LB & $\mathrm{SH}$ & GEO \\
\hline Lower LCOE & 2 & 3 & 3 & 4 & 4 & 3 & 5 & 4 \\
\hline Lower maintenance cost & 4 & 5 & 4 & 4 & 4 & 2 & 4 & 3 \\
\hline Easy installation and use & 5 & 5 & 4 & 5 & 5 & 3 & 3 & 3 \\
\hline Long lifetime & 5 & 5 & 4 & 4 & 4 & 4 & 5 & 4 \\
\hline Battery required & 5 & 2 & 2 & 5 & 5 & 5 & 5 & 5 \\
\hline AC output & 5 & 1 & 5 & 5 & 5 & 5 & 5 & 5 \\
\hline Modularity of systems & 4 & 5 & 4 & 1 & 1 & 1 & 1 & 2 \\
\hline Training required & 3 & 3 & 2 & 5 & 5 & 5 & 4 & 3 \\
\hline Scalability and replicability & 4 & 5 & 2 & 4 & 4 & 2 & 2 & 1 \\
\hline Acceptance and social participation & 4 & 5 & 3 & 4 & 4 & 2 & 5 & 4 \\
\hline Direct social impact & 4 & 5 & 5 & 1 & 1 & 2 & 4 & 3 \\
\hline Low environmental impact & 4 & 5 & 5 & 2 & 2 & 2 & 4 & 5 \\
\hline Generation of employment permanent & 3 & 4 & 4 & 2 & 2 & 2 & 3 & 3 \\
\hline Availability of energy source & 5 & 5 & 4 & 3 & 3 & 1 & 4 & 4 \\
\hline Hybridization ease & 3 & 5 & 5 & 2 & 2 & 3 & 1 & 1 \\
\hline Lower waste generation & 4 & 4 & 4 & 3 & 3 & 4 & 3 & 4 \\
\hline Tariff regulation & 3 & 5 & 4 & 2 & 2 & 4 & 4 & 2 \\
\hline Security against theft & 3 & 3 & 3 & 4 & 4 & 5 & 5 & 5 \\
\hline Required import equipment & 4 & 2 & 2 & 5 & 5 & 5 & 5 & 3 \\
\hline
\end{tabular}

\section{$\mathrm{F}_{6}$ : Maximization of the use of renewable energy technologies for sustainable supply of energy in IRC}

In Table XIV, the precedence constraints have been evaluated by the authors as per the below-mentioned subjects, based on the indicated references.

TABLE XIV. Precedence constraints of $F_{6}$. 
TABLE XV. Calculation of $M$ and $N$ factors.

\begin{tabular}{lccc}
\hline \hline Alternative & $M$ & $N$ & Coefficient \\
\hline ST & $1.0 \times 10^{11}$ & $2.5 \times 10^{5}$ & 2.5 \\
PV & $8.4 \times 10^{10}$ & $8.6 \times 10^{3}$ & 2.5 \\
WE & $1.8 \times 10^{10}$ & $1.8 \times 10^{6}$ & 2 \\
SH & $1.7 \times 10^{10}$ & $1.7 \times 10^{5}$ & 2 \\
GEO & $1.7 \times 10^{10}$ & $9.3 \times 10^{6}$ & 2 \\
FW & $1.8 \times 10^{9}$ & $3.7 \times 10^{6}$ & 1.5 \\
CH & $1.8 \times 10^{9}$ & $3.7 \times 10^{6}$ & 1.5 \\
LB & $3.5 \times 10^{8}$ & $2.2 \times 10^{7}$ & 1 \\
\hline \hline
\end{tabular}

$M$ and $N$ are then calculated (Table XV):

Once the coefficients were applied, this is the final equation for $F_{6}$ :

$$
F_{6}=2.5 \mathrm{ST}+2.5 \mathrm{PV}+2 \mathrm{WE}+2 \mathrm{SH}+2 \mathrm{GEO}+1.5 \mathrm{FW}+1.5 \mathrm{CH}+\mathrm{LB} .
$$

\section{RESULTS AND DISCUSSION}

The Chebysev distance was chosen as the Pareto optimal solution. Table XVI shows the results obtained using Matlab ${ }^{\circledR}$ software for each energy vector. In order to take into account more than one decision maker's point of view, different weights have been considered. Thereby, more or less importance has been given to the studied functions.

For the analysis, two scenarios for each objective function were established: one where the function is the only criterion of interest to the decision makers, for the $F_{1}$ case $(1,0,0,0,0,0)$, and the other,where the function has a higher weight than the others, for the $F_{1}$ case $(5,1,1,1,1,1)$. Then, both cases were compared to the baseline scenario, i.e., giving all functions the same weight: $(1,1,1,1,1,1)$. See Figs. 4-9.

TABLE XVI. Results obtained for minimization of $L_{\infty}$ for each energy alternative and considering different weights (ktoe).

\begin{tabular}{|c|c|c|c|c|c|c|c|c|c|c|c|c|c|}
\hline \multicolumn{6}{|c|}{ Weights } & \multirow[b]{2}{*}{ ST } & \multirow[b]{2}{*}{ PV } & \multirow[b]{2}{*}{ WE } & \multirow[b]{2}{*}{ FW } & \multirow[b]{2}{*}{$\mathrm{CH}$} & \multirow[b]{2}{*}{ LB } & \multirow[b]{2}{*}{ SH } & \multirow[b]{2}{*}{ GEO } \\
\hline $\mathrm{F}_{1}$ & $\mathrm{~F}_{2}$ & $\mathrm{~F}_{3}$ & $\mathrm{~F}_{4}$ & $\mathrm{~F}_{5}$ & $\mathrm{~F}_{6}$ & & & & & & & & \\
\hline 1 & 1 & 1 & 1 & 1 & 1 & 0.909 & 2.673 & 0.407 & 7.407 & 1.885 & 0.888 & 1.929 & 0.130 \\
\hline 1 & 0 & 0 & 0 & 0 & 0 & 1.658 & 3.136 & 0.013 & 12.693 & 2.705 & 1.034 & 3.575 & 0.205 \\
\hline 5 & 1 & 1 & 1 & 1 & 1 & 1.418 & 2.984 & 0.094 & 11.000 & 2.442 & 0.989 & 3.097 & 0.181 \\
\hline 0 & 1 & 0 & 0 & 0 & 0 & 1.658 & 2.644 & 2.393 & 12.693 & 2.705 & 1.034 & 1.687 & 0.205 \\
\hline 1 & 5 & 1 & 1 & 1 & 1 & 1.429 & 2.794 & 0.331 & 11.077 & 2.454 & 0.985 & 3.075 & 0.182 \\
\hline 0 & 0 & 1 & 0 & 0 & 0 & 1.658 & 2.644 & 2.393 & 12.693 & 2.705 & 0.887 & 1.687 & 0.205 \\
\hline 1 & 1 & 5 & 1 & 1 & 1 & 1.417 & 2.870 & 0.288 & 10.998 & 2.442 & 0.988 & 3.016 & 0.181 \\
\hline 0 & 0 & 0 & 1 & 0 & 0 & 1.658 & 2.644 & 0.355 & 12.693 & 2.705 & 1.034 & 3.725 & 0.205 \\
\hline 1 & 1 & 1 & 5 & 1 & 1 & 1.417 & 2.648 & 0.374 & 10.995 & 2.441 & 0.988 & 3.152 & 0.181 \\
\hline 0 & 0 & 0 & 0 & 1 & 0 & 0.100 & 2.644 & 0.013 & 1.700 & 1.000 & 0.740 & 0.500 & 0.050 \\
\hline 1 & 1 & 1 & 1 & 5 & 1 & 0.374 & 2.658 & 0.141 & 3.632 & 1.300 & 0.791 & 0.985 & 0.077 \\
\hline 0 & 0 & 0 & 0 & 0 & 1 & 1.658 & 3.355 & 1.306 & 12.693 & 2.705 & 1.034 & 2.063 & 0.205 \\
\hline 1 & 1 & 1 & 1 & 1 & 5 & 1.420 & 2.870 & 0.493 & 11.017 & 2.445 & 0.983 & 2.818 & 0.181 \\
\hline 1 & 5 & 5 & 5 & 1 & 1 & 1.417 & 2.653 & 0.381 & 10.997 & 2.442 & 0.986 & 3.140 & 0.181 \\
\hline 5 & 1 & 1 & 1 & 1 & 5 & 1.418 & 3.003 & 0.157 & 11.001 & 2.442 & 0.982 & 3.015 & 0.181 \\
\hline
\end{tabular}




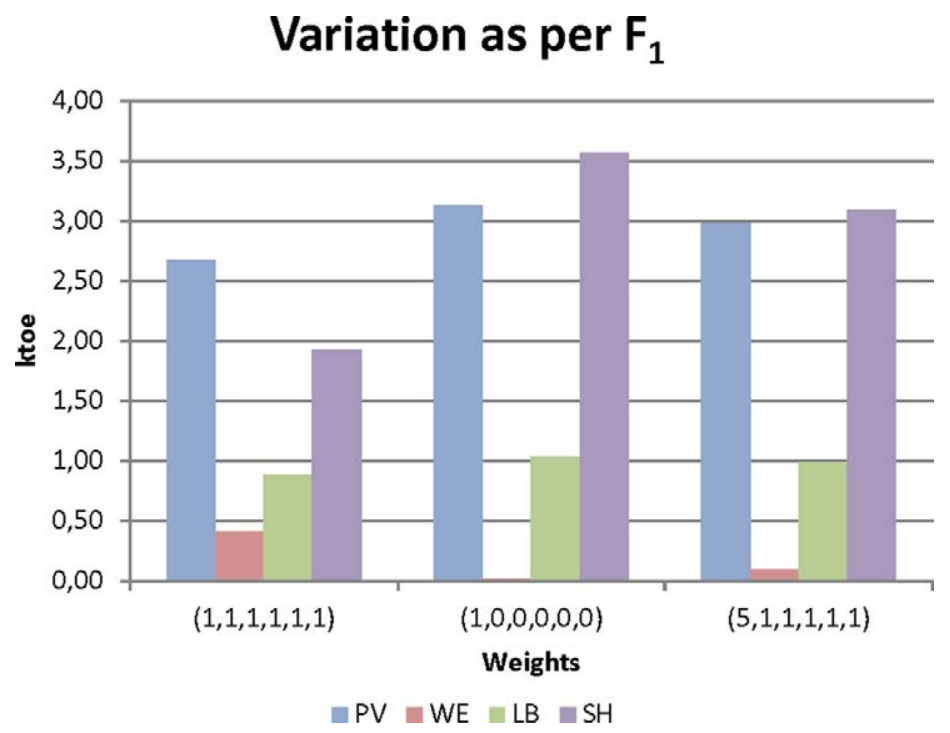

FIG. 4. PV, WE, LB, and SH variation as per weights assigned to $F_{1}$.

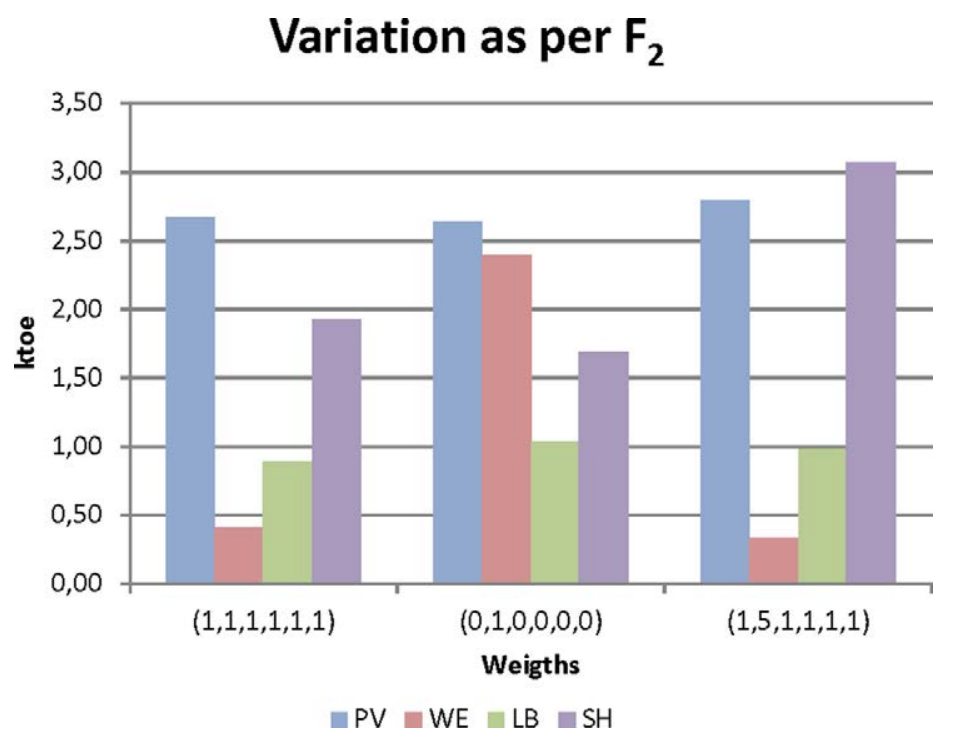

FIG. 5. PV, WE, LB, and $\mathrm{SH}$ variation as per weights assigned to $F_{2}$.

Additionally, two scenarios were analysed: one in which a greater weight to the emissions functions $\left(F_{2}, F_{3}\right.$, and $\left.F_{4}\right)$ is given, and the other giving more importance to encouraging the use of renewable energy and its application for energy supply in IRCs $\left(F_{5}\right.$ and $\left.F_{6}\right)$.

The results show that the energy alternatives that were enhanced according to the weight assigned were ST, FW, CH, and GEO, in most cases. Meanwhile, PV, WE, LB, and SH presented greater variation in the cases and were less favoured in the majority of them.

Figure 8 shows that when considering only the cost function $\left(F_{5}\right)$, the distance values of the variables were minimums. In this case, PV has become the alternative with higher priority. The same occurs when $F_{5}$ has more weight than the others (Fig. 8). In this case, SH ranks second.

Regarding the PV variable, it obtained the lowest values in environmental impact minimization functions. A higher value was obtained when considering only $F_{6}$. When considering only $F_{1}$, and when $F_{1}$ and $F_{6}$ have more weight than the other functions, higher intermediate 


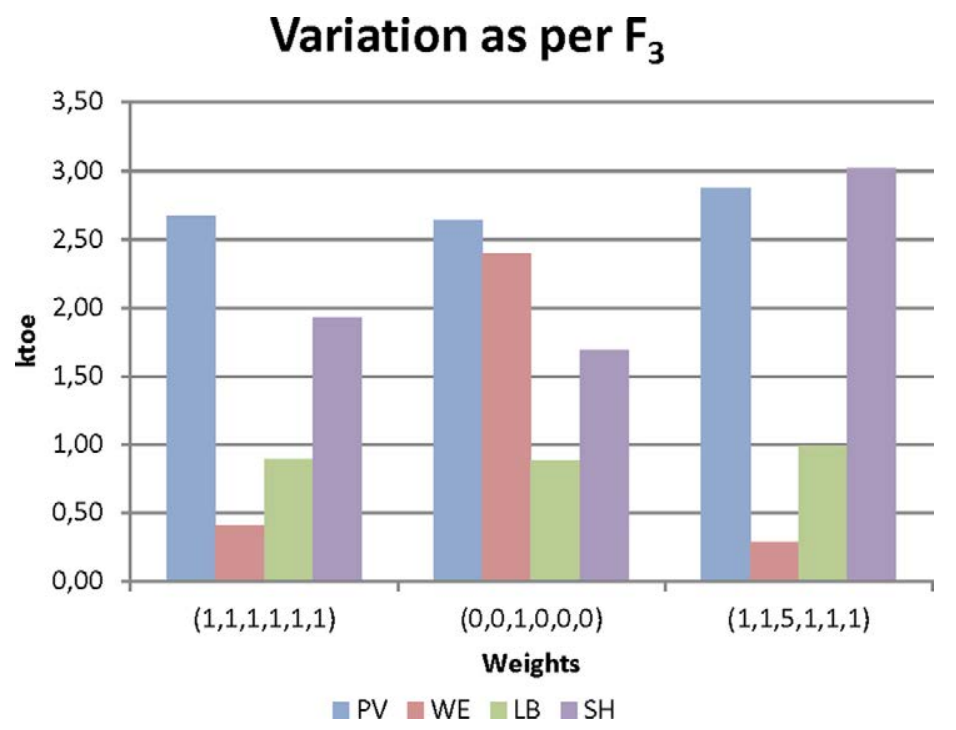

FIG. 6. PV, WE, LB, and $\mathrm{SH}$ variation as per weights assigned to $F_{3}$.

values were obtained (Figs. 4 and 9, respectively). It is worth mentioning that the behaviour of the PV variable was influenced by the amount of fossil fuel that will substitute in mining.

The WE variable obtained higher values when considering only the $F_{2}$ and $F_{3}$ (Figs. 5 and 6). When priority was given to $F_{6}$, this variable obtained its second-best value (Fig. 9).

LB almost has achieved its maximum potential in most scenarios. As with PV, the lower limit was fixed, in this case in order to meet the demand in transport.

The SH variable reached its best values in environmental impact minimization functions. Furthermore, this variable obtained a higher value with $F_{4}$ (Fig. 7).

When the two additional cases are analysed, the trend in both was increased SH. Meanwhile, PV increased only when more importance was given to $F_{1}$ and $F_{6}$. For this last case, WE was decreased. In both cases and for all variables, intermediate values were obtained. On the other hand, when only $F_{6}$ was taken into account, the priority for electric generation was obtained for PV (Fig. 9).

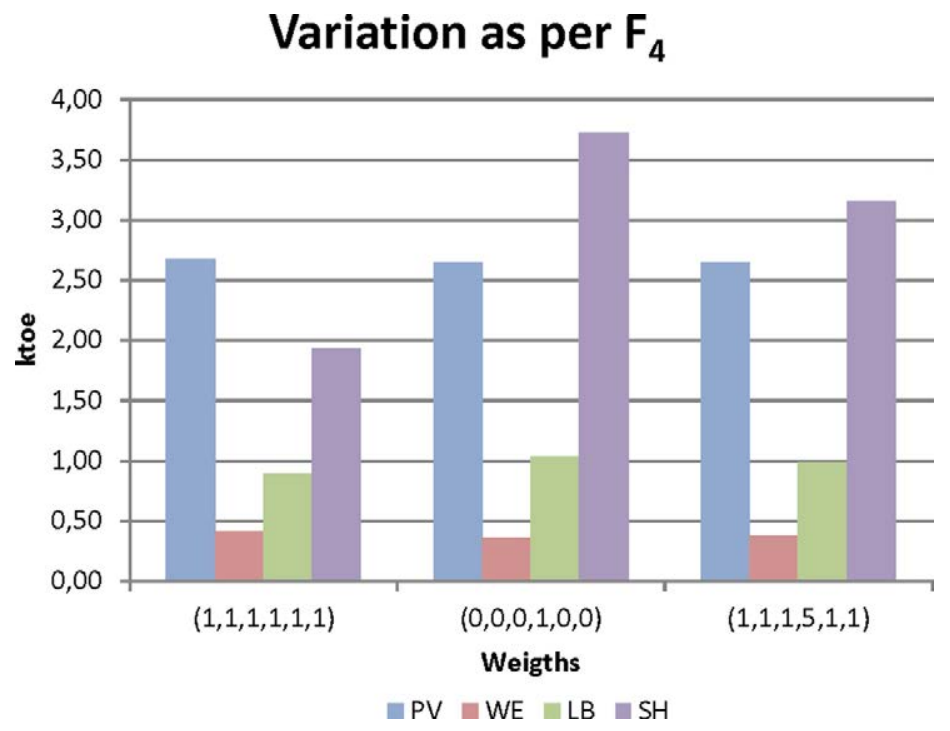

FIG. 7. PV, WE, LB, and $\mathrm{SH}$ variation as per weights assigned to $F_{4}$. 


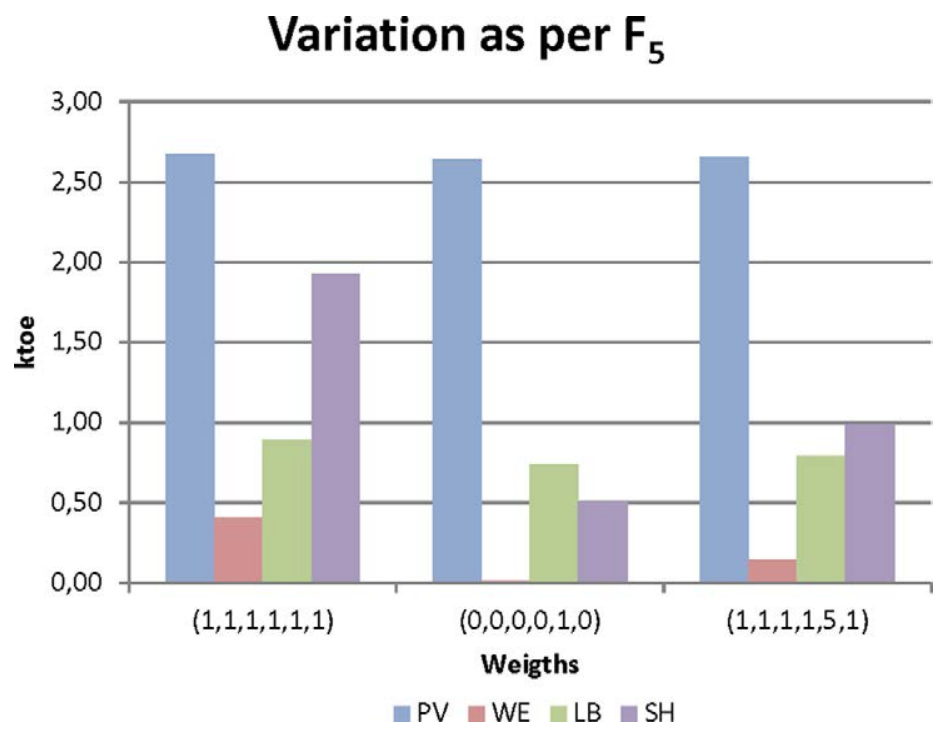

FIG. 8. PV, WE, LB, and $\mathrm{SH}$ variation as per weights assigned to $F_{5}$.

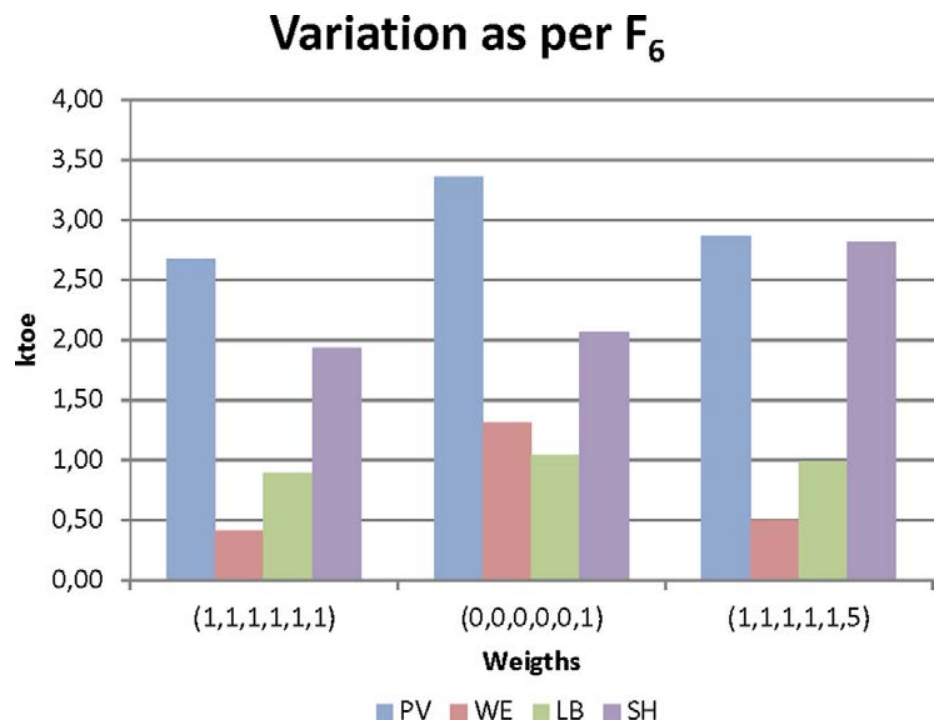

FIG. 9. PV, WE, LB, and $\mathrm{SH}$ variation as per weights assigned to $F_{6}$.

It is worth mentioning that the coefficients in $F_{1}$ and $F_{6}$ are obtained based on a valuation quantitative of technicians and experts, so the reliability of these functions will depend of their know-how about the study area. The other functions use coefficients based on numerical data obtained from government or other official sources. This can become a limitation if information is scarce. Thus, it has to be emphasized that the model is not intended to indicate the "best" solution over the possible alternatives, but to help contextualize relevant pros and cons when supporting decision-making. ${ }^{10}$

\section{CONCLUSIONS}

Today, energy planning models that consider universal access to energy are increasing. This is a priority for developing countries such as Peru. The SEPLAN model proposes a method using MCDM techniques that has allowed the consideration of qualitative and quantitative criteria in planning. Thus, universal access to energy has been incorporated into the 
decision problem, as well as economic and environmental criteria. Thereby, some priorities have been established under various scenarios, i.e., considering possible views of the decision makers.

In the case of the province of Cajamarca, the $\mathrm{ST}, \mathrm{FW}, \mathrm{CH}$, and GEO variables were enhanced in all scenarios, except the one where the cost function is minimized. The values achieved in each case correspond to their maximum possible (in ktoe): $\mathrm{ST}=1.658$; $\mathrm{FW}=12.693 ; \mathrm{CH}=2.705$; and $\mathrm{GEO}=0.205$. Within these alternatives, $\mathrm{ST}$ and GEO are highlighted for the high potential for to expand their use in the province, due to existing experience and availability of the resource.

On the other hand, when $F_{6}$ was favoured, the priority for electric generation was obtained for PV (2.87 ktoe). In the meantime, SH and WE were favoured when environmental impact minimization functions were of major priority.

The SEPLAN model has been designed to be applied in developing countries due to the criteria taken into account in the function $F_{6}$. However, the model can be applied in any region or country in which the six objective functions are considered relevant by decision makers.

Regarding further work, the model proposed could incorporate other objective functions that are relevant for the region in study. Likewise, other types of emissions can be analyzed. It could be of interest to take into account hybrid systems in an objective function, since they are widely used in IRCs.

\section{ACKNOWLEDGMENTS}

The authors would like to thank Mr. Julio Eisman and Mr. José Gabriel Martín from ACCIONA Microenergía Foundation for comments and suggestions that helped to improve this research. The first author also wishes to thank Miss Inma Del Alamo for her support in the revision of the English version of this paper.

${ }^{1}$ International Energy Agency (IEA), http://www.worldenergyoutlook.org/weo2015/ for World Energy Outlook 2015, 2015; accessed 26 May 2016.

${ }^{2}$ Consorcio de Investigación Económica y Social (CIES), http://www.cies.org.pe/sites/default/files/investigaciones/accesouniversal-a-la-energia-y-tecnologias-renovables.pdf, 2016 for Elecciones Perú 2016: Centrando el debate electoral: Acceso Universal a la Energía y Tecnologías Renovables; accessed 21 April 2016.

${ }^{3}$ Instituto Nacional de Estadística e Informática (INEI), https://www.inei.gob.pe/media/MenuRecursivo/publicaciones_digitales/Est/Lib1136/libro.pdf for Censos Nacionales 2007: XI de Población y VI de Vivienda, 2008; accessed 2 February 2016.

${ }^{4}$ Multilateral Investment Fund (MIF), http://www.fomin.org/Portals/0/impact\%20evaluation/PanelesSolares(Peru).pdf for De las velas a la luz eléctrica. El impacto de la electrificación rural, 2014; accessed 19 May 2016.

${ }^{5}$ L. C. Domínguez, "Sustainable and renewable implementation multi-criteria energy model (SRIME)—Case Study: Sri Lanka," Ph.D. thesis (Universidad Europea de Madrid, Madrid, 2015).

${ }^{6}$ S. D. Pohekar and M. Ramachandran, "Application of multi-criteria decision making to sustainable energy planning-A review," Renewable Sustainable Energy Rev. 8, 365-381 (2004).

${ }^{7}$ T. Kaya and C. Kahraman, "Multicriteria decision making in energy planning using a modified fuzzy TOPSIS methodology," Expert Syst. Appl. 38, 6577-6585 (2011)

${ }^{8}$ Organización Latinoamericana de Energía (OLADE), http://www.olade.org/wp-content/uploads/2015/08/Informe-FinalPERU.pdf for Modelos de Mercado, Regulación Económica y Tarifas del Sector Eléctrico en América Latina y el Caribe - Perú, 2013; accessed 1 June 2016.

${ }^{9}$ L. Ferrer-Martí, B. Domenech, A. García-Villoria, and R. Pastor, "A MILP model to design hybrid wind-photovoltaic isolated rural electrification projects in developing countries,” Eur. J. Oper. Res. 226, 293-300 (2013).

${ }^{10}$ F. F. Nerini, M. Howells, M. Bazilian, and M. F. Gomez, "Rural electrification options in the Brazilian Amazon: A multicriteria analysis," Energy Sustainable Dev. 20, 36-48 (2014).

${ }^{11}$ M. Amer and T. U. Daim, "Selection of renewable energy technologies for a developing county: A case of Pakistan," Energy Sustainable Dev. 15, 420-435 (2011).

${ }^{12}$ L. C. Domínguez and F. Marcos, "Sustainable and renewable implementation multi-criteria energy model (SRIME): Case study: Sri Lanka,” Int. J. Energy Environ. Eng. 6, 165 (2015).

${ }^{13}$ J. C. Rojas-Zerpa and J. M. Yusta, "Methodologies, technologies and applications for electric supply planning in rural remote areas," Energy Sustainable Dev. 20, 66-76 (2014).

${ }^{14}$ Ministerio de Energía y Minas (MINEM), http://www.minem.gob.pe/minem/archivos/file/institucional/publicaciones/ InformePlanEnerg\%C3\%ADa2025-\%20281114.pdf for "Plan Energético Nacional 2014 - 2025," 2014; accessed 12 January 2016.

${ }^{15}$ L. García, "Desarrollo de un modelo multicriterio-multiobjetivo de oferta de energías renovables: Aplicación a la comunidad de Madrid," Ph.D. thesis (ETSI de Montes, UPM, Madrid, 2004).

${ }^{16} \mathrm{~F}$. Marcos, "Aplicación de las técnicas multidimensionales a la planificación energética," Energía 11, 97-104 (1985). 
${ }^{17}$ A. Yadoo and H. Cruickshank, "The role for low carbon electrification technologies in poverty reduction and climate change strategies: A focus on renewable energy mini-grids with case studies in Nepal, Peru and Kenya," Energy Policy. 42, 591-602 (2012).

${ }^{18}$ United Nations Development Programme (UNDP), http://hdr.undp.org/sites/default/files/idh2009-peru-vol1-2.pdf for "Human Development Report Peru 2009," 2009; accessed 15 January 2016.

${ }^{19}$ Ministry of Energy and Mining (MINEM), http://dger.minem.gob.pe/ArchivosDger/PNER_2013-2022/PNER-20132022\%20Texto.pdf for "2013-2022 National Rural Electrification Plan,” 2012; accessed 2 February 2016.

${ }^{20} \mathrm{P}$. Lillo, L. Ferrer-Martí, A. Boni, and A. Fernández-Baldor, "Assessing management models for off-grid renewable energy electrification projects using the Human Development approach: Case study in Peru," Energy Sustainable Dev. 25, 17-26 (2015).

${ }^{21}$ ACCIONA Micronergía Perú (AMP). Perfil del proyecto de electrificación de las localidades de la zona rural de Cachachi, La Encañada, Namora, San Pablo, Tumbaden e Ichocan, en la región Cajamarca, empleando energía solar fotovoltaica (Cajamarca, 2011).

${ }^{22}$ P. L. Yu, "A class of solutions for group decision problems," Manage. Sci. 19, 936-946 (1973).

${ }^{23}$ M. Zeleny, Compromise Programming in Multiple Criteria Decision Making (University of South Carolina Press, Columbia, 1973).

${ }^{24}$ C. Romero, http://www.academia.utp.ac.pa/sites/default/files/docente/51/decisiones_multicriterio.pdf for Análisis de las decisiones multicriterio, Isdefe. Madrid, 1996; accessed 15 November 2015.

${ }^{25}$ Organismo Supervisor de la Inversión en Energía y Minería (OSINERGMIN), https://www.osinergmin.gob.pe/seccion/ centro_documental/hidrocarburos/Publicaciones/

BALANCE\%20DE\%20ENERG\%C3\%8DA\%20EN\%20EL\%20PERU\%202014.pdf for "Balance Nacional de Energía 2014, desde la perspectiva de supervisor," 2015; accessed 25 April 2016.

${ }^{26} \mathrm{~L}$. Benitez, "Procedimiento multicriterio-multiobjetivo de planificación energética a comunidades rurales aisladas," Ph.D. thesis (ETSI de Montes, UPM, Madrid, 2015).

${ }^{27}$ Ministerio de Energía y Minas (MINEM), http://www.minem.gob.pe/archivos/legislacion-9ozj22z9ap5zz33zDL_de_promocion_de_la_inversion_para_la_generacion_de_electricidad_con_el_uso_de_energias_renovables_1002.pdf for "Decreto legislativo de promoción de la inversión para la generación de electricidad con el uso de energías renovables: Decreto Legislativo No. 1002," 2010; accessed 5 April 2016.

${ }^{28}$ Organización de las Naciones Unidas para la Alimentación y la Agricultura (FAO), http://www.fao.org/docrep/019/ as415s/as415s.pdf for Estado del Arte y Novedades de la Bioenergía en el Perú, 2011; accessed 15 November 2015.

${ }^{29}$ Servicio Nacional de Meteorología e Hidrología (SENAMHI), http://dger.minem.gob.pe/atlassolar/ATLAS_SOLAR.pdf for Atlas de Energía Solar del Perú, 2003; accessed 20 November 2015.

${ }^{30}$ International Renewable Energy Agency (IRENA), http://www.irena.org/DocumentDownloads/Publications/ RRA_Peru.pdf for "Peru Renewables Readiness Assessment 2014," 2014; accessed 15 February 2016.

${ }^{31}$ Organismo Supervisor de la Inversión en Energía y Minería (OSINERGMIN), http://www2.osinerg.gob.pe/ EnergiasRenovables/contenido/Documentos/1SubastaOffGrid/Bases/BasesDefinitivasRevision.pdf for "Bases definitivas para la subasta de suministro de electricidad con recursos energéticos renovables en áreas no conectadas a red," 2014; accessed 12 March 2015.

${ }^{32}$ Multilateral Investment Fund (MIF), http://www.fomin.org/Portals/0/Topics/BSS_energia_accionaperu.pdf for "Luz en Casa. Acceso a energía sostenible en comunidades rurales,”2013; accessed 15 February 2016.

${ }^{33}$ Ministerio de Energía y Minas (MINEM), http://www.osinergmin.gob.pe/seccion/centro_documental/Institucional/ Estudios_Economicos/Otros-Estudios/Atlas-Eolico/AtlasEolicoLibro.pdf for Atlas Eólico del Perú, 2008; accessed 20 February 2016.

${ }^{34}$ Soluciones Prácticas-ITDG, http://www.solucionespracticas.org.pe/ns_publicaciones for "Soluciones Prácticas en Cajamarca," 2015; accessed 5 April 2016.

${ }^{35}$ A. Díaz, A. Moreno, C. Cabezudo, and A. Castro, Elaboración de un índice de Sostenibilidad de las Cocinas Mejoradas en los departamentos de Tacna, Moquegua, Arequipa, Ayacucho, Huancavelica, Cajamarca y San Martin (EnDev/GIZ, Lima, 2013).

${ }^{36}$ S. Campos and S. Salazar, Diseño de una planta industrial de biodiesel a partir de la semilla de higuerilla en el valle de Condebamba - Cajamarca (Facultad de Ingeniería Industrial, Universidad Privada del Norte. Cajamarca, 2013).

${ }^{37}$ Instituto Nacional de Estadística e Informática (INEI), https://www.inei.gob.pe/media/MenuRecursivo/publicaciones_digitales/Est/Lib1140/cap07.pdf for "Anuario de Estadísticas Ambientales 2013, Capítulo 7, Energía, Minería e Hidrocarburos," 2013; accessed 15 March 2016.

${ }^{38}$ Instituto Nacional de Estadística e Informática (INEI), http://proyectos.inei.gob.pe/web/biblioineipub/bancopub/Est/ Lib1039/libro.pdf for "Perú: Estimaciones y proyecciones de población departamental por años calendario y edades simples 1995-2025," 2010; accessed 20 February 2016.

${ }^{39}$ S. A. Hidrandina, http://www.osinergmin.gob.pe/newweb/uploads/Publico/OficinaComunicaciones/EventosRealizados/ ForoCajamarca/2/Atencion\%20de\%20demanda\%20Cajamarca.pdf for "I Foro Regional de Energía de Cajamarca," 2014; accessed 20 February 2016.

${ }^{40}$ C. Orbegozo and R. Arivilca, Energía Solar Térmica. Manual Técnico para Termas Solares (Green Energy Consultoría y Servicios SRL, Lima, 2010).

${ }^{41}$ Agencia de Cooperación Internacional del Japón (JICA), http://open_jicareport.jica.go.jp/pdf/12048567.pdf for "Plan maestro para el desarrollo de la energía geotérmica en el Perú,” 2012; accessed 20 February 2016.

${ }^{42}$ Soluciones Prácticas-ITDG, www.solucionespracticas.org.pe/Descargar/1531/16285 for "Microaerogeneradores de 100 y 500 W. Modelos IT-PE-100 y SP-500,” 2008; accessed 22 February 2016.

${ }^{43}$ Departamento de Energía, EE.UU., http://apps2.eere.energy.gov/wind/windexchange/pdfs/small_wind/small_wind_guide_spanish.pdf for "Sistemas eólicos pequeños para generación de electricidad," 2002; accessed 22 February 2016.

${ }^{44}$ Organización de las Naciones Unidas para la Agricultura y la Alimentación (FAO), "Análisis de recursos biomásicos leñosos y de residuos para uso combustible," in FAO, Bioenergía y seguridad alimentaria "BEFS" El análisis de BEFS para el Perú (Oficina de Intercambio de Conocimientos, Investigación y Extensión FAO, Roma, 2010), pp. 71-95. 
${ }^{45}$ F. López, Potencial hidroenergético en la zona norte del Perú (Dirección General de Energía y Minas, San Martin, 2014).

${ }^{46}$ Gobierno Regional de Cajamarca, http://zeeot.regioncajamarca.gob.pe/sites/default/files/HIDROLOGIA.pdf for "Estudio Hidrológico de la Región Cajamarca 2010-2011,”2011; accessed 22 February 2016.

${ }^{47}$ Soluciones Prácticas-ITDG. Evaluación de Recursos Hidroenegéticos (Soluciones Prácticas, Lima, 2010), ISBN: 978 9972-47-206-0.

${ }^{48}$ A. Pérez, G. Montero, and R. Ayala, "Simulación en Aspen de la combustión de mezclas diesel-biodiesel," Ing. Invest. Tecnol. XVI (número 1), 83-92 (2015).

${ }^{49}$ Organismo Supervisor de la Inversión en Energía y Minería (OSINERGMIN), http://www.osinergmin.gob.pe/seccion/ centro_documental/Institucional/Estudios_Economicos/RAES/RAES_Electricidad_Noviembre_2014_OEE.pdf for "Reporte de Análisis Económico Sectorial Electricidad Año 3 - № 4. Oficina de Estudios Económicos - OEE," 2014; accessed 22 February 2016.

${ }^{50}$ Ministerio de Energía y Minas (MINEM), http://www.minem.gob.pe/minem/archivos/Cap\%C3\%83\%C2\%ADtulo2_\%20Estad\%C3\%83\%C2\%ADstica\%20El\%C3\%83\%C2\%A9ctrica\%20por\%20Regiones\%202010(1).pdf for "Estadística Eléctrica por Regiones 2010," 2010; accessed 2 February 2016.

${ }^{51}$ Consejo Nacional de Ambiente (CONAM), http://bibliotecavirtual.minam.gob.pe/biam/bitstream/id/583/BIV00133.pdf for "Indicadores Ambientales de Cajamarca. Serie Indicadores Ambientales No. 6," 2005; accessed 01 March 2016.

${ }^{52}$ Ministerio del Ambiente (MINAM). Proyecto Segunda Comunicación Nacional de Cambio Climático, http://sinia.minam.gob.pe/documentos/inventario-nacional-integrado-emisiones-gases-efecto-invernadero-peru for "Inventario Nacional Integrado de Gases de Efecto Invernadero del Perú en el Año 2000,” 2009; accessed 15 March 2016.

${ }^{53}$ M. Pehnt, "Dynamic life cycle assessment (LCA) of renewable energy technologies," Renewable Energy 31, 55-71 (2006).

${ }^{54}$ International Renewable Energy Agency (IRENA), http://www.irena.org/DocumentDownloads/Publications/ IRENA_REmap_Transport_working_paper_2016.pdf for “The Renewable Route to Sustainable Transport - A Working Paper based on Remap," 2016; accessed September 2016.

${ }^{55}$ E. Buyukkaya, "Effects of biodiesel on a DI diesel engine performance, emission and combustion characteristics," Fuel 89, 3099-3105 (2010).

${ }^{56}$ International Renewable Energy Agency (IRENA), http://www.irena.org/documentdownloads/publications/irena_re_power_costs_2014_report.pdf for "Renewable Power Generation Costs in 2014," 2015; accessed 13 May 2016. 